\title{
Concentration statistics for conservative solute transport in river estuaries
}

Galešić, Morena

Doctoral thesis / Disertacija

2018

Degree Grantor / Ustanova koja je dodijelila akademski / stručni stupanj:

University of Split, Faculty of Civil Engineering, Architecture and Geodesy / Sveučilište u Splitu, Fakultet građevinarstva, arhitekture i geodezije

https://doi.org/10.31534/DocT.044.GalM

Permanent link / Trajna poveznica: https://urn.nsk.hr/urn:nbn:hr:123:389037

Rights / Prava: In copyright/Zaštićeno autorskim pravom.

Download date / Datum preuzimanja: 2023-04-26

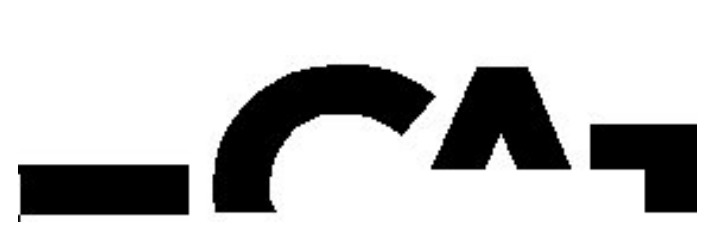

Repository / Repozitorij:

FCEAG Repository - Repository of the Faculty of Civil Engineering, Architecture and Geodesy, University of Split

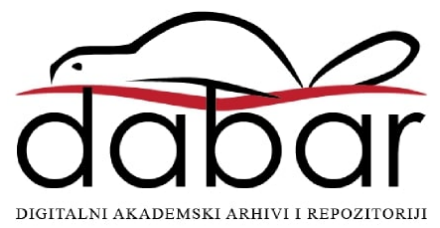




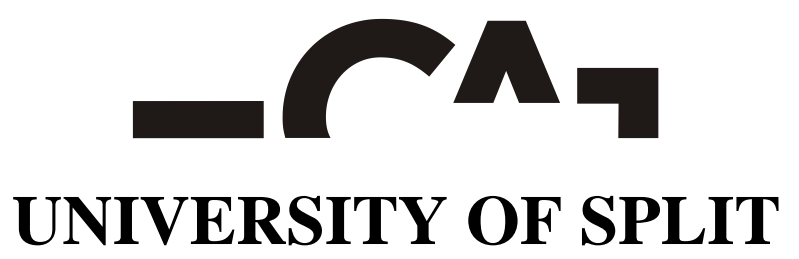

FACULTY OF CIVIL ENGINEERING, ARCHITECTURE AND GEODESY

Morena Galešić, mag.ing.aedif.

\section{Concentration statistics for}

conservative solute transport in river estuaries

Doctoral dissertation

Split, 2018. 

Morena Galešić, mag.ing.aedif.

Serial number: 044

This doctoral dissertation has been submitted for evaluation to Faculty of Civil Engineering, Architecture and Geodesy of University of Split, for the purpose of obtaining the doctoral degree in the field of Technical sciences, scientific field of Civil engineering.

Supervisor: Prof. Roko Andričević, $\mathrm{PhD}$

The committee for evaluation:

Prof. Nikša Jajac, PhD

Prof. Hrvoje Gotovac, PhD

Prof. Darko Koračin, PhD

Prof. Vladimir Cvetković, $\mathrm{PhD}$

Prof. Goran Lončar, PhD 

The defense committee:

Prof. Nikša Jajac, PhD

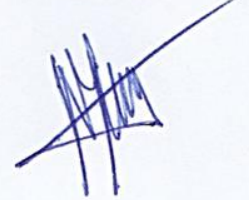

Prof. Hrvoje Gotovac, $\mathrm{PhD}$

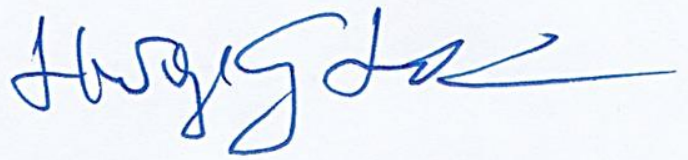

Prof. Darko Koračin, $\mathrm{PhD}$

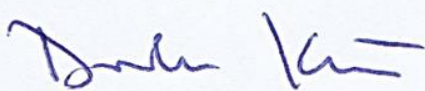

Prof. Vladimir Cvetković, PhD
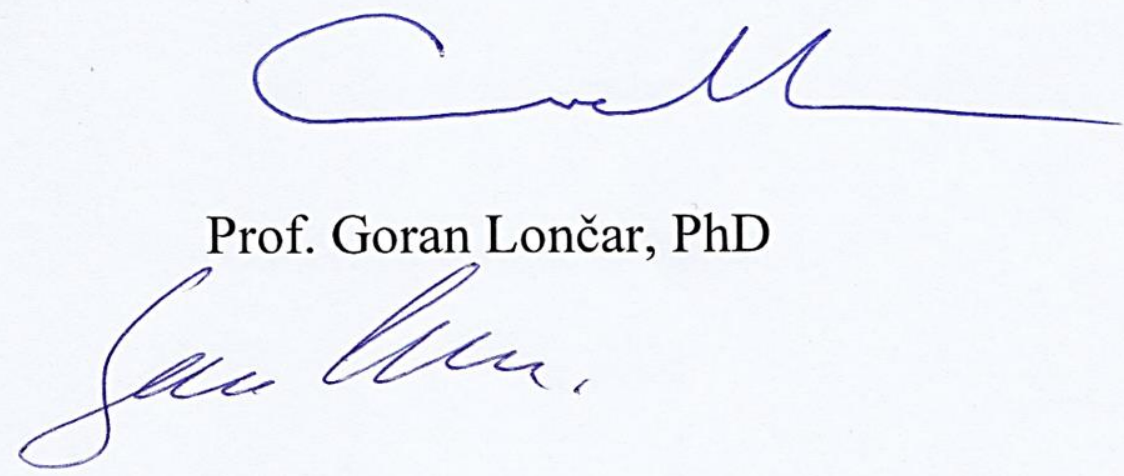

The doctoral dissertation has been defended on February 16, 2018.

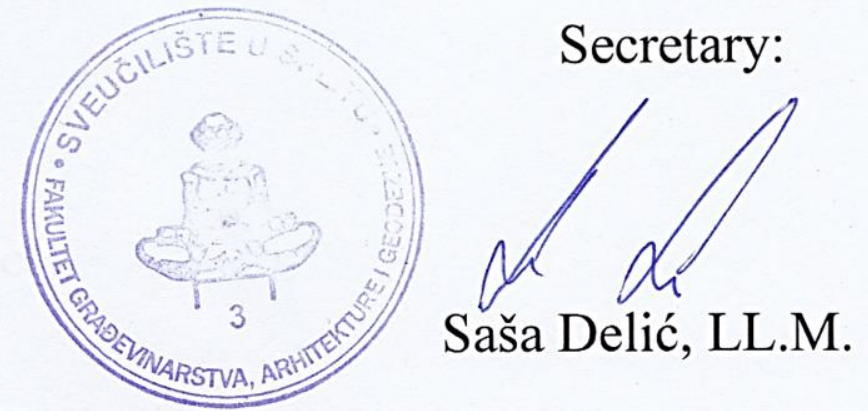



This dissertation contains:

160 pages

83 figures

2 tables

154 references 

Posvećeno mojoj majci Marici

"Things are only impossible until they're not."

Capt. Jean Luc Picard (H. L. Shearer), 1988. 



\section{Acknowledgments}

This thesis has been predominantly supported by the Faculty of Civil Engineering, Architecture and Geodesy (FGAG) at University of Split. However, additional funding was enabled through the project HR.3.2.01-0262, Risk assessment of the pollution caused by rivers and discharges in the coastal area (project CPoRT - Coastal Pollution Risk Tool) co-funded by European Union from European Social Fund.

I would like to thank all those individuals who contributed to this thesis, my research and personal growth that has been achieved during all these years.

First of all, my greatest thanks go to my supervisor, prof. Roko Andričević without whom I might have never started this crazy adventure of pursuing the scientific truths. He

pushed my limits beyond the levels that I ever imagined reaching, and luckily, he had the power of focusing my extremely diffusive energy patterns. I am particularly thankful for all the time he invested in our work, especially when considering how busy he was.

The second most important player had demanding roles of being both partners in my research and life as well. The title of an incredibly understanding and supportive husband goes to prof. Vladimir Divić with whom I had some of the most breaking through discussions, and who endured all my roller coaster moments of this research.

I am very thankful to all my colleagues from the Department of Applied hydraulics (FGAG). Thanks go to prof. Hrvoje Gotovac with whom I worked very closely for the master thesis, during which some of the initial parts of my heterogeneous scientific path were modeled. Furthermore, my thanks go to prof. Veljko Srzić for his consistent challenging of obtained results which gave a significant spice to overall dynamics of the research. He also took part of my teaching duties during the writing of this thesis, for which I am very thankful. Prof. Mijo Vranješ is the best jokester there is, so when I needed to brighten up my day, there he was with an advanced level of sarcasm to force the laugh out of me. Nevertheless, he never failed to teach me something new about the sea and the coast.

Special thanks here go to the dean of FGAG, prof. Boris Trogrlic for his support and trust in my work during the application and implementation of the CPORT project. The continuous supply of exciting activities from his side did my job at FGAG fulfilling and empowering. 
I would like to thank prof. Ramiro Neves from MARETEC group at Instituto Superior Técnico in Lisbon, for his hospitality to Vladimir and me during our research there. Among many colleagues in Lisbon that we had the opportunity to work with, special thanks go to prof. Lígia Pinto, prof. Marcos Mateus, Gianfranco Calise and Guilherme Franz.

I would like to express my great gratitude to the reviewers of this thesis, prof. Nikša Jajac, prof. Hrvoje Gotovac, prof. Vladimir Cvetković, prof. Darko Koračin and prof. Goran Lončar. They all gave useful comments and questions which improved the thesis.

Besides, I want to thank all the other individuals that I had the opportunity to meet, to know better and who made an impact on my work and me. Many of them had become friends, and some had become my second family. They are Margita Perišić, Petra Šimundić, Ivan Racetin, Lea Levi, prof. Mirela Galić, Mirna Raić, Mak Kišević, Amer Smailbegović, Jean Charles Belley, Francois Kaisin, Jelena Kilić, Valentina Kuzmanić, Antonija Prlj, Josip Peroš, Ana Jeličić, Toni Kekez, Ivan Lovrinović, Goran Vlastelica, Petra Šunjić, Mario Parat, Slobodan Bonković, prof. Ljiljana Šerić, Hrvoje Mihanović, Luka Malenica, Marina Sunara Kusić, Marko Mimica and Ana Maria Topić

There is a special thank you for my friend Robert Šakić Troglić who always believed I could do more, and who has still experienced my worries and excitations no matter how far away he was. Furthermore, my unique maid of honor, Marina Tavra has always been there for me, and the word "thanks" barely meets the requirements for what she deserves.

Finally, I want to thank my family, starting from my parents, Marica and Milivoj, through the clans of my three big sisters or the parliament of secondary moms: Mirjana, Emilija and Romana, to my mother and grandmother in law, Branka and Mira. Special thanks are given to all of them for their continuous support and understanding.

Thanks to my cat Jackie for ongoing lessons on curiosity and ability to focus. Iowe my gratitude to all the people and cat mentioned above, along with potentially any other unnamed beings who have influenced my work and me! 


\title{
Morena Galešić, mag.ing.aedif.
}

\section{Concentration statistics for conservative solute transport in river estuaries}

\begin{abstract}
:
When addressing water quality issues in coastal zone management, rivers are considered to be among the most significant pathways for waterborne solute transport. On the other hand, estuaries are some of the most productive environments on Earth and multiple interests from different stakeholders put additional pressure on such an ecosystem. Estuaries are thus among the most critical areas for pollution hazard and a lot of research is being conducted in order to ensure their sustainable development.
\end{abstract}

The flow in an estuary is characterized by a mixing process, which results from a combination of small-scale turbulent diffusion and larger-scale variation of mean advective velocities. Scalar concentration in such turbulent flows is a random variable, which requires a statistical approach. This thesis focuses on the development of two analytical methodologies for obtaining concentration statistics of a conservative contaminant generated by a steady river flow in a salt-wedge estuary. Analytically obtained concentration statistics, which include concentration moments, probability density function defined in point, spatially integrated moments, and expected mass fraction, significantly reduce the costs and time needed for obtaining the measured data. Although these analytical models lack the level of detail possessed by sophisticated numerical models, they can generate results within seconds and with little preprocessing. Therefore, the developed analytical models represent screening tools for obtaining concentration statistics of pollution in estuaries, and can be used by stakeholders of different backgrounds.

While investigating various validation procedures for the developed analytical models, and using the case study of the Žrnovnica River estuary, near Split, Croatia, several new findings were obtained. For instance, a new model for mean velocity attenuation was proposed and verified. Furthermore, a new proxy concentration was 
tested and, combined with numerical modeling, it delivered qualitative verification of analytically obtained concentration statistics. The analytical model for point concentration statistics was implemented in a user-friendly computer application (CPoRT) and presented to different stakeholders as a screening tool.

Finally, the most important scientific contributions of this thesis are the development of the semi-analytical approach for obtaining concentration statistics in a river-dominated estuary, application of proxy concentration using the salinity data and the introduction of the expected mass fraction as a significant novelty to address the pollution loading to coastal waters.

Keywords: estuaries, river generated pollution, concentration statistics, probability density function (PDF), expected mass fraction (EMF) 
Morena Galešić, mag.ing.aedif.

\section{Prostorna distribucija koncentracije zagađenja kao rezultat pronosa $\mathbf{u}$ ušćima rijeka}

\section{Sažetak:}

Prilikom analize kakvoće vode u okviru upravljanja obalnim područjima, rijeke se smatraju jednim od najznačajnijih izvora zagađenja. Nadalje, ušća pripadaju najbogatijim ekosustavima na Zemlji, što ih čini podložnima dodatnim pritiscima različitih interesa. Stoga su ušća iznimno osjetljiva područja te se brojna istraživanja provode radi osiguravanja njihova održivog razvoja.

Tok u ušćima obilježen je procesom miješanja koje predstavlja rezultat djelovanja turbulentne difuzije na maloj skali te varijabilnosti advektivne srednje brzine na većoj skali. U takvim turbulentnim tokovima skalarna koncentracija jest slučajna varijabla, što zahtijeva odgovarajući statistički pristup. Ova disertacija usredotočena je na razvoj dviju analitičkih metodologija za izračun statističkih parametara koncentracije zagađenja koje je nastalo uslijed konzervativnog i stacionarnog pronosa u stratificiranom ušću. Tako dobiveni parametri uključuju statističke momente koncentracije, pripadajuće funkcije gustoće vjerojatnosti, prostorno integrirane momente koncentracije te funkcije očekivanog udjela mase. Ako se izračunaju analitički, navedene veličine znatno smanjuju moguće troškove mjerenja. Razvijeni analitički modeli nemaju razlučivost poput sofisticiranih numeričkih modela, ali mogu brzo pružiti tražene vrijednosti uz malo pretprocesiranja. Zaključno, navedeni analitički modeli predstavljaju alate za brzu procjenu zagađenja u nekom ušću, te ih kao takve mogu upotrebljavati dionici s različitim predznanjem.

Prilikom validacijskih postupaka za razvijene metodologije na pilot-području ušća rijeke Žrnovnice, pored Splita (Hrvatska), došlo je do novih spoznaja. Predložen je i verificiran novi model smanjenja srednje brzine toka. Uz navedeno, predložena je i nova proxy koncentracija (zamjena za koncentraciju zagađenja), koja je u kombinaciji s 
rezultatima numeričkog modeliranja omogućila kvalitativnu verifikaciju statističkih parametara dobivenih analitičkim modelima. Naknadno je analitički model za izračun točkastih statističkih parametara ugrađen u računalnu aplikaciju (CPoRT), a ista je predstavljena različitim dionicima upravo kao alat za brzu procjenu potencijalnog zagađenja u ušćima.

Zaključno, najvažniji znanstveni doprinosi ove disertacije su razvoj poluanalitičkog pristupa za izračun statističkih parametara koncentracije u ušću dominiranom tokom rijeke, primjena proxy koncentracije korištenjem podataka o slanosti, primjena koncepta očekivanog udjela mase kao značajne novine prilikom analize opterećenja zagađenjem na priobalne vode.

Ključne riječi: ušća, zagađenje uzrokovano rijekama, statistički parametri koncentracije, funkcija gustoće vjerojatnosti, očekivani udio mase 


\section{Contents}

Abstract xiii

Contents $\quad$ xvii

$\begin{array}{ll}\text { List of Figures } & \text { xxi }\end{array}$

List of Tables $\quad$ xxv

$\begin{array}{lc}\text { List of Abbreviations } & \text { xxvii }\end{array}$

$\begin{array}{ll}\text { List of Symbols } & \text { xxix }\end{array}$

$\begin{array}{ll}\text { Preface } & \text { xxxi }\end{array}$

1 Introduction 1

1.1 Problem setting ...................... 2

1.2 General overview . . . . . . . . . . . . . . . . . . . . 4

1.3 Research questions . . . . . . . . . . . . . . 6

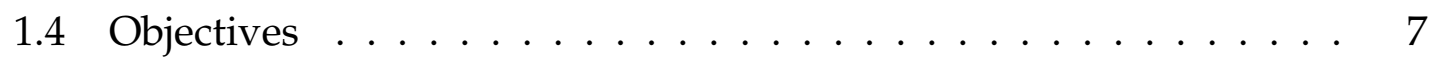

1.5 Scientific contribution $\ldots \ldots \ldots . \ldots \ldots$

2 Point concentration statistics $\quad 11$

2.1 Methodology for concentration moments development . . . . . . . 11

2.1.1 Concentration moments for the specific case of constant mean velocity ...................... 16

2.1.2 Concentration moments for a non-uniform mean velocity . 17

2.2 Probability density function for pollution concentration . . . . . . . 19

2.3 Moment inversion to obtain the PDF . . . . . . . . . . . . . . . 22

2.3.1 Moment-matching method . . . . . . . . . . . . 22

2.3.2 Maximum entropy method - MaxEnt . . . . . . . . . . 23

3 Spatially integrated concentration statistics 31

3.1 Expected mass fraction $-\mathrm{EMF} \ldots \ldots \ldots$. . . . . . . . . . . 31

3.1 .1 EMF background . . . . . . . . . . . . . . 32 
3.1.2 EMF for the case of contaminant plume with continuous

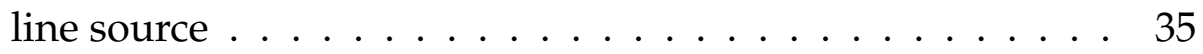

3.2 Direct integration of PDFs . . . . . . . . . . . . . 36

3.3 Spatially integrated concentration moments $-K_{n} \ldots \ldots \ldots$

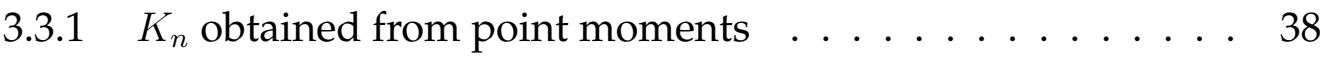

3.3.2 $K_{n}$ obtained using a recursive model $\ldots \ldots \ldots \ldots \ldots 4$

3.3.3 Moment inversion to obtain the EMF . . . . . . . . . . . . 44

4 Field measurements and numerical modeling 47

4.1 Case study - Žrnovnica River estuary . . . . . . . . . . . . 47

4.2 Field measurements . . . . . . . . . . . . . . . . . . . 50

4.2.1 Setup for velocity measurements . . . . . . . . . . . . 51

4.2.2 Setup for salinity measurements . . . . . . . . . . . 53

4.3 Numerical modeling . . . . . . . . . . . . . . . . . 57

4.3.1 Random walk particle tracking approach . . . . . . . . 58

4.3.2 Complex modeling approach - MOHID . . . . . . . . . 62

5 Results 73

5.1 Analytical modeling . . . . . . . . . . . . . . 73

5.1.1 Single point concentration statistics . . . . . . . . . 74

5.1.2 Integrated concentration statistics $\ldots \ldots \ldots \ldots$. . . . 84

5.2 Measurements . . . . . . . . . . . . . . . . . . . . . 91

5.2 .1 Velocity measurements . . . . . . . . . . . . . . . . . 91

5.2 .2 Salinity measurements . . . . . . . . . . . . . . . . . 93

5.3 Numerical modeling . . . . . . . . . . . . . . . . . . 100

5.3.1 Random walk particle tracking model . . . . . . . . . . . 101

5.3 .2 MOHID . . . . . . . . . . . . . . . . . . . 103

5.4 Comparisons and discussion $\ldots \ldots \ldots$. . . . . . . . . 108

5.4.1 Mean velocity attenuation model . . . . . . . . . . . . . 108

5.4.2 Comparison of the first two concentration moments between the analytical and the RWPT numerical models . . . . . . 109

5.4.3 Comparison between salinity measurements, MOHID results, and the analytical solution . . . . . . . . . . . . 111

5.4.4 Potential limitation of the near-field approximation . . . . . 114

5.4.5 Comparison between beta distribution EMFs obtained by inversion of their measured and analytically defined moments . . . . . . . . . . . . . . . . . . . . . . . 115

5.4.6 The EMF concept as a potential contaminant dilution measure in an estuary . . . . . . . . . . . . 116 
6 Practical application of the research conducted 119

6.1 Project synopsis . . . . . . . . . . . . . . . . . . . . . 119

6.2 CPoRT application . . . . . . . . . . . . . . . . 122

6.3 Stakeholder's involvement . . . . . . . . . . . . . . . . 125

7 Conclusions and directions for future progress 129

7.1 Conclusions . . . . . . . . . . . . . . . . . . . . . . . . . . 129

7.2 Directions for future progress . . . . . . . . . . . . . 131

$\begin{array}{ll}\text { A ADE in terms of moments } & 133\end{array}$

B Detailed development of pollution concentration moments 135

B.1 The specific case of constant mean velocity . . . . . . . . . . . 135

B.2 The case of non-uniform mean velocity . . . . . . . . . . . 137

C Detailed development of spatially integrated moments 143

C.1 The specific case of constant mean velocity . . . . . . . . . . . . . . 143

C.2 The case of non-uniform mean velocity . . . . . . . . . . . . . . 144

C.3 Spatially integrated moments in source . . . . . . . . . . . . . 144

$\begin{array}{ll}\text { Bibliography } & 147\end{array}$ 



\section{List of Figures}

1.1 Example of an estuary highly impacted by rivers . . . . . . . . . 2

1.2 Problem formulation scheme .............. 3

1.3 Research plan .................. 7

2.1 Proposed function for threshold concentration . . . . . . . . . . . 15

2.2 Impact of different attenuation coefficients on the velocity field . . 17

2.3 Examples of concentration central moments . . . . . . . . . . . . . . 20

2.4 Examples for concentration beta distribution PDF . . . . . . . . . 22

2.5 Example of MaxEnt distribution estimate . . . . . . . . . . . . 26

2.6 Computational time for the MaxEnt algorithm . . . . . . . . . . 28

2.7 The concentration PDFs obtained by MaxEnt . . . . . . . . . . 28

3.1 An arbitrary realization of contaminant cloud release . . . . . . 33

3.2 An arbitrary realization of contaminant plume release . . . . . . . . 36

3.3 Schematic representation of spatially integrated pollution concentration PDFs . . . . . . . . . . . . . . . . 37

3.4 EMFs obtained by direct integration . . . . . . . . . . . . 38

3.5 Volume and mass as a function of the section . . . . . . . . . . . 39

3.6 Spatially integrated moments and corresponding EMF moments obtained by integration of point moments . . . . . . . . . . . . . . 40

3.7 Spatially integrated moments obtained by recursive solution . . . . 43

3.8 EMF examples as a function of dimensionless time . . . . . . . . . . 44

3.9 EMF examples for constant and non-uniform mean velocity . . . . 45

4.1 Žrnovnica River estuary . . . . . . . . . . . . . . . . . . . . 48

4.2 3D depth distribution of the case study area . . . . . . . . . . . . . . . . . . . . . . . . . . . . . .

4.3 Water levels example data . . . . . . . . . . . . . . . . . . . . 49

4.4 Žrnovnica flows example data . . . . . . . . . . . . . . . . . . 50

4.5 Velocity measurements procedure . . . . . . . . . . . . . 51

4.6 Velocity and salinity measurement instruments . . . . . . . . . . . 52

4.7 Salinity measurement setup . . . . . . . . . . . . . . . . . . 54

4.8 Salinity probe test in the laboratory . . . . . . . . . . . . . 55

4.9 Salinity measurement setup in the field . . . . . . . . . . . 55 
4.10 Recorded positions for different salinity measurement campaigns . 58

4.11 Random walk particle tracking scheme . . . . . . . . . . . . . 59

4.12 Concentration calculation in the RWPT model . . . . . . . . . . 60

4.13 An example of a RWPT simulation . . . . . . . . . . . . . . . . 60

4.14 Schematic representation of the MOHID Water framework . . . . . 64

4.15 Coastline for the case study . . . . . . . . . . . . . . . 68

4.16 Geometry setup for the Žrnovnica estuary in MOHID . . . . . . . . 69

4.17 Vertical discretization for the MOHID 3D model . . . . . . . . . . 71

5.1 Concentration mean distribution . . . . . . . . . . . . . . . . . 74

5.2 Concentration standard moments . . . . . . . . . . . . . . . . . . 75

5.3 The influence of molecular diffusion on the second central moment 77

5.4 The influence of molecular diffusion on the third central moment . 78

5.5 The influence of molecular diffusion on the fourth central moment $\quad 78$

5.6 The influence of mean velocity attenuation on mean concentration 79

5.7 The influence of mean velocity attenuation on the second central moment . . . . . . . . . . . . . . . . . 80

5.8 The influence of mean velocity attenuation on the third central moment . . . . . . . . . . . . . . . . 81

5.9 The influence of mean velocity attenuation on the fourth central moment . . . . . . . . . . . . . . . . . . 81

5.10 The concentration PDFs and CDFs for $\nu=0 \ldots \ldots$. . . . . 82

5.11 The concentration PDFs and CDFs for $\nu=0.002 \mathrm{~m}^{-1} \ldots \ldots$. . . . 83

5.12 The concentration PDFs and CDFs for $\nu=0.01 \mathrm{~m}^{-1} \ldots \ldots$. . . . 83

5.13 EMFs obtained by direct spatial integration of point Beta distribution PDFs . . . . . . . . . . . . . . . . . . . . 85

5.14 Contaminant mass fraction exhibiting concentration levels above limit value . . . . . . . . . . . . . . . . . 85

5.15 Spatially integrated moments $K_{n}$ for different conditions . . . . . . 87

5.16 EMF moments $\tilde{m}_{n}$ for different conditions . . . . . . . . . . . . . 88

5.17 EMFs obtained by inversion of their moments at different distances from the source . . . . . . . . . . . . . . . . . . . 89

5.18 Contaminant mass fraction exhibiting concentration levels above the limit value obtained by spatially integrated moments . . . . . . 90

5.19 The positions of velocity measurements used for the evaluation of the velocity attenuation coefficient . . . . . . . . . . . . . . 92

5.20 Measured velocities fitted with the proposed mean velocity model 92

5.21 The salinity profile near the river mouth . . . . . . . . . . . . . 93 
5.22 Proxy concentration at two characteristic points for the first measurement campaign . . . . . . . . . . . . . . . . . 94

5.23 Proxy mean concentration and variance field for the first measurement campaign . . . . . . . . . . . . . . . . . . . 94

5.24 Proxy concentration at two characteristic points for the second measurement campaign . . . . . . . . . . . . . . . . . 95

5.25 Proxy mean concentration and variance field for the second measurement campaign . . . . . . . . . . . . . . . . . 95

5.26 Proxy concentration at two characteristic points for the third measurement campaign . . . . . . . . . . . . . . . . 96

5.27 Proxy mean concentration and variance field for the third measurement campaign . . . . . . . . . . . . . . . . 96

5.28 PDFs of proxy concentration for three example points . . . . . . . . 97

5.29 The convergence of proxy concentration statistics relative to the measuring interval . . . . . . . . . . . . . . . . . 98

5.30 Proxy concentration at two characteristic points for the fourth measurement campaign . . . . . . . . . . . . . . . 99

5.31 Proxy mean concentration and variance field for the fourth measurement campaign . . . . . . . . . . . . . . . . . . 99

5.32 Proxy mean concentration and variance at two profiles perpendicular to the centerline . . . . . . . . . . . . . . . . 100

5.33 Convergence tests for the RWPT model . . . . . . . . . . . . . . 101

5.34 Different snapshots of an arbitrary RWPT realization . . . . . . . 102

5.35 RWPT concentration mean and variance surface plots . . . . . . . 103

5.36 A snapshot of MOHID results for the velocity field . . . . . . . . . 104

5.37 A snapshot of MOHID results for the salinity field . . . . . . . . . 105

5.38 A snapshot of MOHID results for Lagrangian transport . . . . . . . 106

5.39 A snapshot of the MOHID vertical profile . . . . . . . . . . . . . 107

5.40 Velocity model verification . . . . . . . . . . . . . . . . . 108

5.41 Mean concentration comparison . . . . . . . . . . . . . . . . . . 109

5.42 Concentration variance comparison . . . . . . . . . . . . . 110

5.43 Comparison of the concentration mean and variance between analytical, measured and MOHID results at measurement points' positions . . . . . . . . . . . . . . . . . . 111

5.44 Comparison of the mean concentration field obtained by the analytical model, by measurements, and by the MOHID model . . . . 112

5.45 Potential definition of the limit distance for the near field approximation . . . . . . . . . . . . . . . . . . . . 114 
xxiv

5.46 Comparison of analytical EMF and EMF obtained from proxy concentration measurements at two cross-sections . . . . . . . . . . 115

5.47 The seaward extension of the dilution process for different estimated loadings . . . . . . . . . . . . . . . . . . 117

6.1 Schematic of project workflow . . . . . . . . . . . . . . . . 120

6.2 Field measurements example . . . . . . . . . . . . . . . . 120

6.3 CPoRT project logo . . . . . . . . . . . . . . . . 121

6.4 CPoRT application graphical interface . . . . . . . . . . . . . . . . . . . . . . . . . . . . . . .

6.5 Illustrative results from the CPoRT application . . . . . . . . . . . . 124

6.6 The research problem scheme within a broader framework . . . . 125

6.7 The stakeholders' workshop . . . . . . . . . . . . . . . 126 


\section{List of Tables}

4.1 The properties of velocity measurement campaigns. . . . . . . . . . 52

4.2 The properties of salinity measurement campaigns. . . . . . . . . 57 



\section{List of Abbreviations}

$\begin{array}{ll}\text { WFD } & \text { Water Framework Directive } \\ \text { NBWD } & \text { New Bathing Water Directive } \\ \text { MSFD } & \text { Marine Strategy Framework Directive } \\ \text { PDF } & \text { Probability Density Function } \\ \text { EMF } & \text { Expected Mass Fraction } \\ \text { MOHID } & \text { Modelo Hidrodinâmico } \\ \text { MARETEC } & \text { Marine, Environment and Technology Research Centre } \\ \text { CPoRT } & \text { Coastal Polution Risk Tool } \\ \text { ADE } & \text { Advection Diffusion Equation } \\ \text { MaxEnt } & \text { Maximum Entropy method } \\ \text { RWPT } & \text { Random Walk Particle Tracking } \\ \text { IOF } & \text { Institute of Oceanography and Fisheries } \\ \text { PSU } & \text { Practical Salinity Unit } \\ \text { GOTM } & \text { General Ocean Turbulence Model } \\ \text { HDF } & \text { Hierarchical Data Format } \\ \text { MSDI } & \text { Marine Spatial Data Infrastructure }\end{array}$





\section{List of Symbols}

$\begin{array}{lll}c(\mathbf{x}, t) & \text { scalar solute concentration } & \mathrm{kgm}^{-3} \\ C_{0} & \text { source concentration } & \mathrm{kgm}^{-3} \\ \bar{c}(\mathbf{x}, t) & \text { mean concentration } & \mathrm{m}^{-3} \\ c_{p}(\mathbf{x}, t) & \text { normalized concentration proxy } & \mathrm{one}^{-1} \\ S(\mathbf{x}, t) & \text { salinity } & \mathrm{gkg}^{-1} \\ e_{m} & \text { coefficient of molecular diffusion } & \mathrm{m}^{2} \mathrm{~s}^{-1} \\ e_{t} & \text { spatial variance growth rate } & \mathrm{m}^{2} \mathrm{~s}^{-1} \\ \lambda & \text { concentration gradient scale (contaminant microscale) } & \mathrm{m} \\ \nu & \text { mean velocity attenuation coefficient } & \mathrm{m}^{-1} \\ M & \text { total mass of contaminant release } & \mathrm{kg}^{-1} \\ \dot{m} & \text { steady mass flux of contaminant } & \mathrm{kgs}^{-1} \\ L & \text { steady mass loading of contaminant through the plane } & \\ - & \text { normal to the flow direction } x & \mathrm{kgm}^{-2} \\ \bar{v}(\mathbf{x}, t) & \text { mean velocity vector } & \mathrm{ms}^{-1} \\ U\left[U_{x}, 0,0\right] & \text { downstream direction mean velocity } & \mathrm{ms}^{-1} \\ \nu & \text { mean velocity attenuation coefficient } & \mathrm{m}^{-1} \\ m_{n}(\mathbf{x}, t) & \text { standard concentration moments of point PDF } & \mathrm{kg}^{\mathrm{n}} \mathrm{m}^{-3 \mathrm{n}} \\ \mu_{n}(\mathbf{x}, t) & \text { central concentration moments of point PDF } & \mathrm{kg}^{\mathrm{n}} \mathrm{m}^{-3 \mathrm{n}} \\ K_{n}(t) & \text { spatially integrated concentration moments } & \mathrm{kg}^{\mathrm{n}} \mathrm{m}^{-3(\mathrm{n}-1)} \\ \tilde{m}_{n}(t) & \text { moments of expected mass fraction PDF } & \mathrm{kg}^{\mathrm{n}} \mathrm{m}^{-3 \mathrm{n}} \\ & & \end{array}$





\section{Preface}

The level of population is constantly growing in coastal areas, and so are the threats arising from that process. The already sensitive ecosystem is being pushed to its limits. Given that it is quite a complex system, there is no easy way of addressing all occurring problems, or devising an all-encompassing solution.

The focus of this thesis is a specific issue of pollution delivered by rivers into coastal zones. The issue embodies an everlasting battle between ensuring the European and national environmental standards for protecting the ecosystem and its biodiversity on the one hand, and balancing the economic and social development of coastal zones on the other. The pollution generated by rivers and coastal outfalls represents one of the biggest threats to coastal water quality. Different types of pollutants are carried by those sources into coastal zones, including the results of population growth, agricultural and industrial activities from inland. This research is focused on passive contaminants found in an upper layer of a stratified, river-dominated estuary. In a quick assessment, specifically one conducted during a short period of time, nutrients (like nitrogen and phosphorous) might be treated as non-reactive contaminants. This type of estimate for nutrients might be very useful when their adverse effects on the ecosystem are taken into account. The effects are emphasized when certain levels of concentration are exceeded. Among general water quality reduction issues, eutrophication is one of the most dangerous outcomes when overdosing ambient water with nutrients. Water quality issues and their impact on the estuarine ecosystem have been studied in recent decades using different approaches and focusing on specific problems or integrating the entire system. Nevertheless, obtaining measurements, specifically repeated measurements in order to analyze concentration statistics in such a complex system has always been very tedious and expensive, even impossible in certain conditions. Following the proposed problem formulation, this research analyzes the potential of implementing the evaluation of a stochastic advection-diffusion equation which would enable obtaining certain pollution concentration statistics for continuous and steady discharges in a river-dominated estuary.

Chapter 1 presents an outline of the problem formulation regarding the distribution of pollution concentration statistics for the near field of an estuary. Due to 
economic, social and environmental significance of estuaries and coastal areas in general, there is a lot of research from different domains covering the subject. An overview of literature on estuaries and dilution processes in other environmental flows is given.

In Chapter 2, a new methodology for obtaining pollution concentration moments is developed from the fundamental advection-diffusion equation for the case of continuous, steady, conservative transport. A similar approach has previously been applied in other environmental flows (atmosphere and groundwater). However, the methodology is adapted to the problem of the near-field zone for riverdominated stratified estuaries. The developed moments are further investigated for non-uniform mean velocity. All embedded approximations are explained along with all possibilities and limitations of the proposed methodology. The second part of the chapter presents the utilization of developed concentration moments in the form of pollution concentration probability density function at the point (PDF). A PDF is an indispensable part of a classical approach to pollution risk assessment of a certain body of water or any other medium.

In Chapter 3, the expected mass fraction (EMF) is introduced as a potential new measure of the dilution process in the contaminant plume generated by rivers. The EMF intrinsically represents a function very similar to the PDF, but instead of probability, it represents the fraction of mass containing a certain level of concentration. Following that definition, the EMF can be obtained by integrating the PDFs for different concentration intervals in all space or all time. However, this research presents a more direct approach to obtaining the EMF using the concept of spatially integrated concentration moments.

Chapter 4 comprises different approaches implemented in order to obtain a certain level of validation for the developed methodologies. This chapter also includes several field measurement campaigns in the Žrnovnica River case study. Furthermore, two different numerical models are used - the first one simplified and developed by the author, and the other one more complex, using the existing solution for hydrodynamical modeling (MOHID).

Chapter 5 reveals the results of the developed methodologies, along with the results of conducted numerical modeling and field measurements explained in Chapter 4. An emphasis is put on the comparison of modeled and measured data to the data obtained by the developed methodologies.

Chapter 6 demonstrates a practical implementation of the developed methodology, specifically for obtaining the concentration statistical moments and PDFs in arbitrary points. For this purpose, a user-friendly computer application named 
CPoRT (Coastal Pollution Risk Assessment Tool) is developed as a simple screening tool. The application is a product of a project funded by the European Social Fund (ESF) within a research grant program supporting young scientists in Croatia. The project has been closely intertwined with the research conducted in this thesis, which is why the developed methodology has already been presented to relevant stakeholders.

Finally, Chapter 7 produces a summary and a concluding discussion which include the scientific impact of the thesis results, the possibilities and limitations of the developed methodology, and feedback from relevant stakeholders. More importantly, speculations are given on fruitful directions for further research and potential product developments. 



\section{Chapter 1}

\section{Introduction}

Coastal waters are continuously in danger of pollution originating from various sources inland, whether it is a product of agriculture, industry or wastewater from urban areas. According to an estimate by Cohen et al. [32], around 44\% of the world's population lived within $150 \mathrm{~km}$ from the coast, and it is now expected to increase above 75\% within $100 \mathrm{~km}$ by 2025 [17]. The population's activities generate a large environmental impact on surface water, especially on vulnerable, closed water ecosystems with naturally less circulation available. Most of the coastal waters in Croatia and beyond, the entire Adriatic Sea basically, form a closed basin, which makes it susceptible to water quality issues due to limited water circulation.

Estuaries are among the most productive environments on Earth and are considered to be coastal marine areas [7], since they have predominately marine features. They represent intersections of different natural and even social gradients, e.g. salt water intrusion and tidal force from the sea, wind stresses in all effective directions, river flow gradients, and finally human impact through its coast-directed vectors, whether they represent urban, agricultural, industrial, or recreational activities [58]. From a technical point of view, estuaries are complex ecosystems characterized by interdependence of hydrodynamic and ecological processes [123], which lays out some of the key research questions to be addressed in this thesis.

This chapter shows the blueprints for research conducted in the area of conservative solute (i.e. potential pollution) transport behavior in an estuary generated by continuous river inflow. Starting by explaining the problem setting (Section 1.1), then providing a general overview of related research (Section 1.2), and ending by stating the research questions (Section 1.3) and objectives (Section 1.4), the chapter delivers the expected scientific contribution (Section 1.5). 


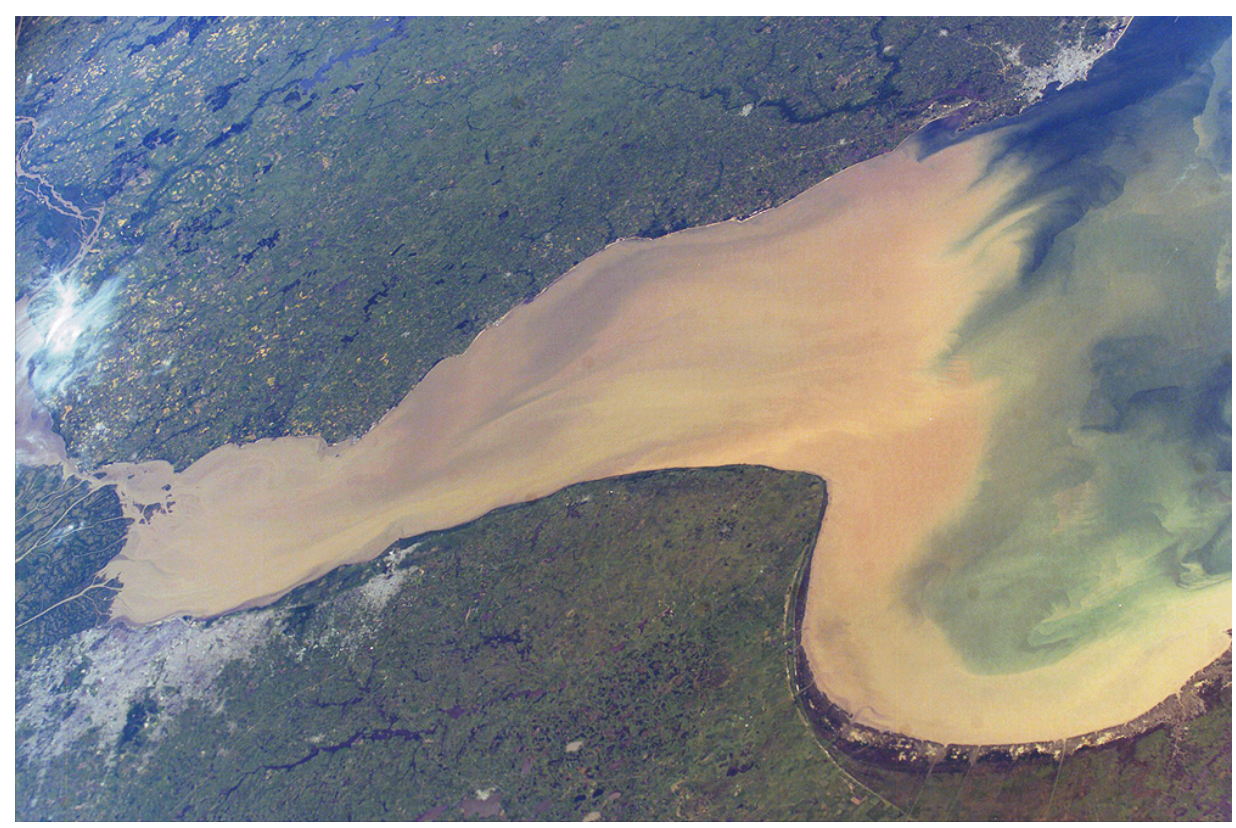

FIgURE 1.1: The Rio de la Plata estuary highly impacted by the rivers Parana and Uruguay (courtesy of NASA).

\subsection{Problem setting}

Rivers are considered to be some of the most influential hydrological pathways for waterborne pollution transport, carrying nutrients, bacteria, heavy metals, or any other type of contaminants. An extreme example is the muddy estuary of the Rio de la Plata (Fig. 1.1), where the rivers Parana and Uruguay flow into the rich estuary and the Atlantic Ocean. This snapshot indicates complex mixing where thick sediment plume serves as a marker for the fresh water masses [108]. There are many different aspects of such an ecosystem to be investigated and some of those are reviewed in Section 1.2.

The issue to be addressed by this thesis is the following one: a steady mass flux of contaminant $(\dot{m})$ is delivered by a river to an estuary system dominated by a stream flow mean velocity $\left(U_{x}\right)$ which is aligned with the $x$-axis of the coordinate system (Fig. 1.2). This concept is based on the fact that in the near-field zone the interactions between the tidal and the residual flow with a weak tidal force are rather limited or absent [30].

Mixing in the near-field zone of an estuary results from a combination of smallscale turbulent diffusion and larger-scale variation of the mean advective velocities [53]. Advection and turbulent diffusion between streamlines cause an equilibrium process of mixing exchange. 


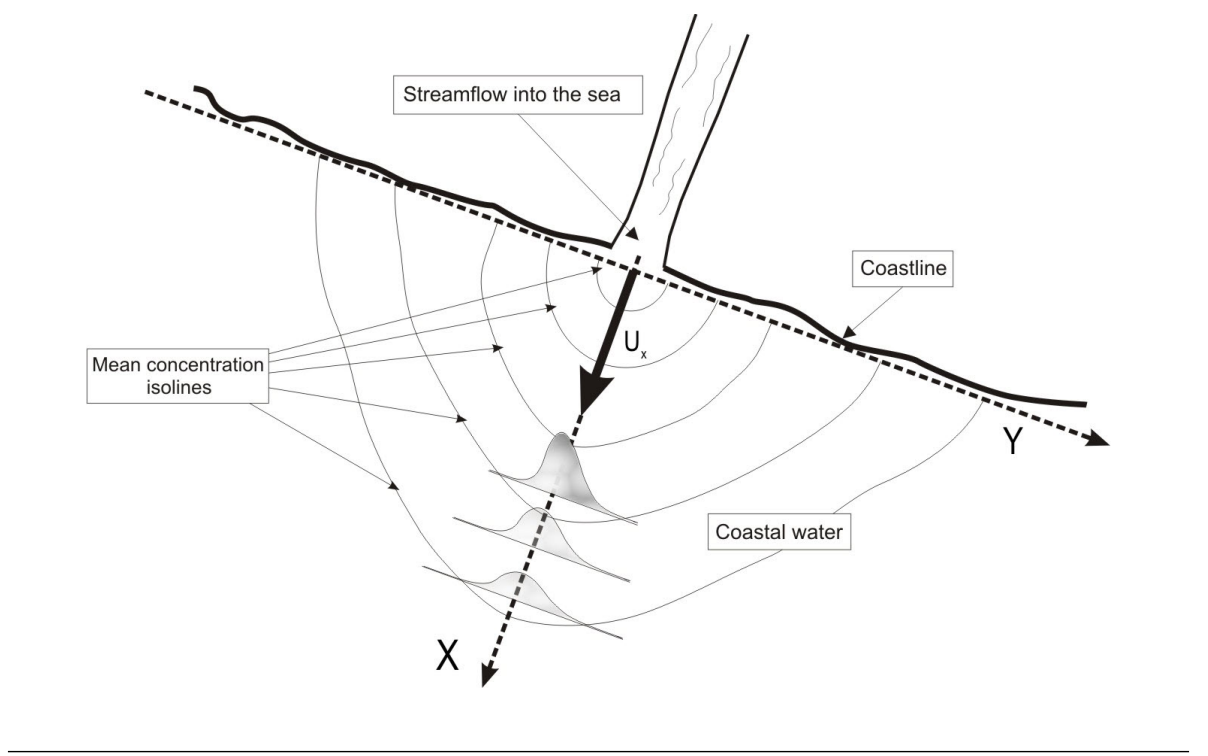

FIGURE 1.2: Problem formulation scheme.

For the observed incompressible and steady flow of conservative substances, the mass conservation is described by the fundamental advection-diffusion equation (ADE):

$$
\frac{\partial c(\mathbf{x}, t)}{\partial t}+\nabla \cdot[v(\mathbf{x}, t) c(\mathbf{x}, t)]=e_{m} \nabla^{2} c(\mathbf{x}, t)
$$

where $c(\mathbf{x}, t)$ is the scalar concentration in units of mass per unit volume, $v(\mathbf{x}, t)$ is the instantaneous flow velocity at point $\mathbf{x}$ and time $t$, and $e_{m}$ is the molecular diffusion coefficient. In turbulent flows, the scalar concentration can be treated as a random variable due to the high irregularity of turbulent velocity fluctuations, as well as of initial and boundary conditions. Such intrinsic randomness of the physical quantities prevents one from using the deterministic theory when predicting concentration field behavior. Therefore, the development of multiple means of concentration statistics presentations will be the focus of this work due to their varied practical interests and thus a basic description of these means is given.

To characterize the reduction of concentration, a probability density function (PDF) is considered:

$$
p(c ; \mathbf{x}, t) d c=\operatorname{prob}\{c \leq c(\mathbf{x}, t) \leq c+d c\}
$$


Furthermore, an adequate approximation of such a function can be found in terms of lower order central moments:

$$
\mu_{n}(\mathbf{x}, t)=\int_{0}^{\infty}\left(c-m_{1}\right)^{n} p(c ; \mathbf{x}, t) d c
$$

where $m_{n}(\mathbf{x}, t)$

$$
m_{n}(\mathbf{x}, t)=\int_{0}^{\infty} c^{n} p(c ; \mathbf{x}, t) d c
$$

are the concentration standard moments. Through moment inversion, the concentration PDF can be reconstructed. Hence, in order to obtain such important information (PDF), the methodology for obtaining concentration moments in an estuary system is developed and presented in the first part of Chapter 2, while PDFs are constructed in the second part to demonstrate their application. However, the probability density function is defined in point, which, on the one hand, makes it very difficult to measure or experimentally determine and, on the other hand, gives a rather limited prediction. It might be useful to find a less experimentally demanding measure of dilution as described by [76, 154]. This measure of dilution would converge with fewer realizations and be less sensitive to spatial or temporal resolution, which is why it is further developed and discussed within integrated concentration statistics in Chapter 3.

\subsection{General overview}

In the last several decades, coastal zone management has been an extremely active and demanding area of research, as well as practical problems. As previously discussed, the focus of this research is to develop analytical methodologies for obtaining contaminant concentration statistics, which would provide screening tools in estuaries. The quick assessment results would potentially rationalize the monitoring plans and additional, more complex modeling. These outcomes are directly related to the issues of preserving water quality in an estuary and its associated coastal waters, while predicting the potential threat brought by rivers. Environmental standards regarding water quality are defined by international and local regulations, among which the European Commission directives represent very important ones, especially for member countries and soon-to-be members. The Water Framework Directive (WFD) [48] represents an umbrella 
directive covering multiple guidelines for water resources management and assessment of ecological status of water bodies. Along with the WFD, there have been guidelines for bathing water quality to safeguard the public health since the 1970s and those are currently implemented as the revised New Bathing Water Directive (NBWD) [46]. Both of those directives, as well as the new Marine Strategy Framework Directive (MSFD) [47] in particular, contribute to the achievement of good ecological status of coastal waters. Therefore, the sheer number of extensive and different paths of research in this area is not surprising.

Most of the pollution pressuring coastal waters originates in various human activities on the mainland, where different nutrients (e.g. nitrogen, phosphorous, etc.) frequently have a major impact on the ecological status of the recipient water body $[72,134]$. The production of nutrients, particularly nitrogen, has significantly increased in the last 150 years, resulting in their accumulation and severe changes in natural recipients $[61,60,150]$. For instance, due to the natural vulnerability of the closed Baltic Sea, multiple analyses have been performed to define nutrient pathways from land to coastal waters [38, 42]. Such studies have shown rivers to be among the largest pollution generators due to their comprehensive accumulation of all agricultural, municipal, and industrial byproducts inland [114]. It has also been demonstrated that in rather closed water bodies, prone to low circulation, the nitrogen volume generated by human activities inland is substantially higher than the one naturally occurring in the environment by atmospheric sedimentation or other processes $[67,71,79]$.

In addition, the estuarine ecosystem is intrinsically complex due to many different aspects. The intercepting influences of river discharge, tidal forces, windinduced stress and water density variations result in turbulent mixing [53, 64]. Each estuary has its own specific characteristics, ranging from its geological background to its morphology or present land use, which is why its behavior is not easy to predict. Rather useful systematization and classification of estuaries with various guidelines is given in [123]. Moreover, a discussion about the potential use of morphological classification to enable prediction in ungauged estuaries is given in [122]. Among various topics related to estuary issues, the most relevant to the focus of this thesis are those covering hydrodynamics and modeling of transport processes in estuaries. Some authors focused on flow and transport dynamics mostly under tidal force [18, 22, 113]. The Lagrangian numerical model has been used to describe hydrodynamics and pollution behavior in coastal zones [19]. Comprehensive research on pollutant dispersion in the near-shore region 
has been conducted on the Spanish Mediterranean coast [115], and similar research was also done on the Alexandria coast [104], both of these showing dominant effects of long-shore currents on the transport. Most of conducted research that can be found in literature is site-specific and closely related to its geographic situation, including unique pollution generators such as agriculture [90], plastic debris [81], or a printing factory [87].

The discussed research problem, as explained in problem setting (Section 1.1), belongs to the domain of analyzing the pollution dilution, specifically in an estuary characterized by a dispersion process, as thoroughly reviewed by Chatwin and Allen in [23]. Hence, physical behavior of such a process is somewhat similar to those in other environmental flows such as contaminant jets in the atmosphere or pollution transport processes in aquifers. Furthermore, the methodologies developed and presented in this thesis follow a similar methodology, already applied in the atmosphere $[25,124,140]$ and in groundwater $[4,5,68]$.

\subsection{Research questions}

This research aims to investigate whether an initial concentration of a conservative pollutant that is continuously discharged by a river for a definite period of time can be used to predict the level of dilution in a near field of an estuary.

The key research questions to be addressed are the following ones:

- To what degree is it possible to define the level of pollution loading in an estuary that is brought in by a river?

- How can the contaminant concentration reduction in an estuary, characterized by complex physical processes, be described in a simplified manner?

- Is it possible to use the concept of expected mass fraction as a dilution measure for a passive contaminant in an estuary?

- How can the developed analytical methods be implemented into a userfriendly product which could be used in practice? 


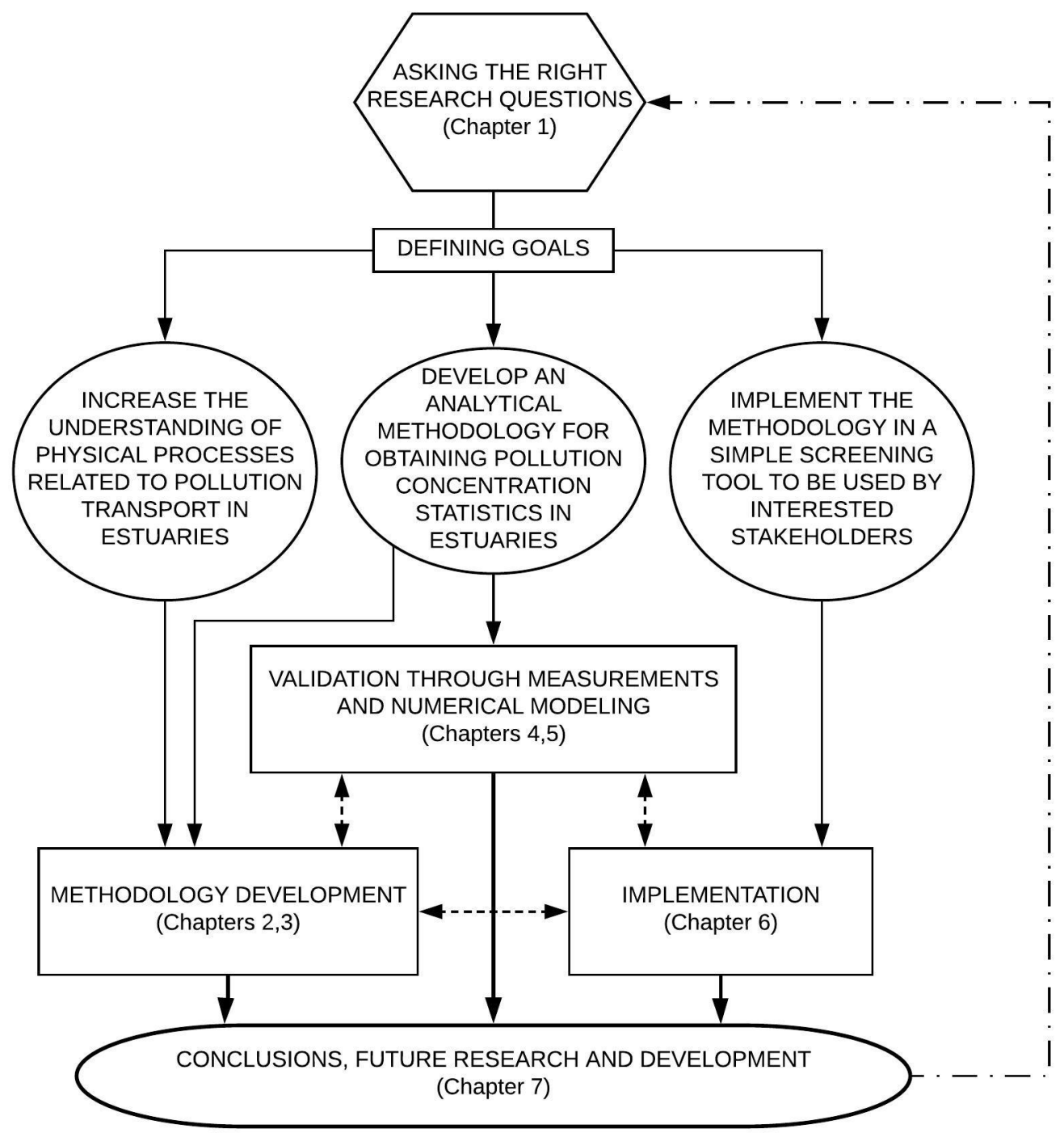

FIGURE 1.3: Schematic of the research plan.

\subsection{Objectives}

Without doubt, raising questions is important and it represents the first step on the scientific journey of reaching scientific truths, by achieving the right goals or objectives defined by the same questions.

In order to properly address them and build a research plan, the main objectives of this thesis are given below:

- Methodology evaluation for the definition of pollution loading in an estuary, caused by river discharges. 
- Development of an analytical model for the prediction of pollution concentration statistics in estuaries, by using 2D representation of the advection-diffusion process.

- Evaluation of the expected mass fraction approach and an analysis of its applicability to the estuary system.

- Validation of the developed analytical methodology by implementing a functional mixture of numerical modeling and field measurements.

- Implementation of the developed methodology in a user-friendly product, i.e. a simple screening tool for predicting pollution loading in an estuary.

Fig. 1.3 is a systematic preview of the research plan, its interlinked activities, and their connections to the associated chapters.

\subsection{Scientific contribution}

The main contributions of this research are in the form of developing new approaches and adapting the existing ones from other environmental flows to the ecosystem of an estuary.

The contribution itself can be illustrated in several points:

- The adapted and developed methodology for obtaining concentration statistics for conservative solute transport is applied for the first time to surface waters for a continuous steady line source.

- The developed analytical model will enable relatively quick prediction of concentration statistics for the potential conservative pollution by knowing only the initial concentration at the source and the mean flow velocity.

- A new simplified mathematical model is defined to represent the changes in the mean flow velocity field that occur downstream from the source. A new experimentally-obtained coefficient is used for this purpose.

- The salinity is for the first time used as a proxy for measuring the statistics of mixing processes in an estuary, which makes it possible to obtain the properties of physical behavior directly in the field. The suggested approach would enable significantly easier and less expensive measurements in contrast with tracer experiments. 
- The concept of expected mass fraction brings a significant novelty to the analysis of potential pollutant loading in the water body of an estuary. Furthermore, different approaches to obtain the EMF are used, including indirect field measurement via a salinity proxy.

An important aspect and a practical contribution of this research is comprised in its potential to be used by a wider audience of different stakeholders interested in coastal waters near rivers or other surface discharges. The developed package - a simple screening tool (CPoRT) - enables potential end users a hands-on approach to tackling rather complex estuarine system issues. CPoRT was developed within the research project (HR.3.2.01-0262, Risk assessment of the pollution caused by rivers and discharges in coastal area) which was part of this thesis and started a dialogue with the stakeholders. It represents merely a starting point toward a more inclusive and holistic approach to addressing many occurring challenges that the future has already brought to coastal zone management. Resilient communities and ecosystems are certainly the pillars of sustainable development and there is a lot of research and work to be done in that direction. 



\section{Chapter 2}

\section{Point concentration statistics}

This chapter develops an analytical model for obtaining pollution concentration moments for a steady flow in estuaries by applying the formulation previously used in other environmental flows. The moments are then used to obtain PDFs and CDFs. The methodology and the scientific contribution of the chapter has been published as part of the following paper:

- Galesic M., Andricevic R., Gotovac H., Srzic V. (2016). Concentration statistics of solute transport for the near field zone of an estuary, Advances in Water Resources. 94:424-440.

The first part of the chapter focuses on the development of analytical methodology for obtaining concentration moments and is described in Section 2.1 for the case of uniform and non-uniform mean velocity field, while Section 2.2 and Section 2.3 produce its application in the form of PDFs and CDFs.

\subsection{Methodology for concentration moments devel- opment}

Following the problem setting explained in Section 1.1, demonstrated here is the formulation for the analytical model of pollution concentration moments. The random variable $c(\mathbf{x}, t)$ is the pollution concentration value which is very difficult to obtain by laboratory or field measurements. Following the conditions of continuum treatment explained by [154], the probabilistic physical characteristics represent the composition of the mixture of host and contaminant fluids as the mass of contaminant per unit volume. Furthermore, the concentration is subject to change in time and space by two different physical processes, namely advective transport and molecular diffusion. The conservation of the contaminant mass is described by Eq. (1.1). By transferring the contaminant even when the fluid as a whole is at rest, molecular diffusion is irrespective of the velocity 
field. However, advection greatly enhances the transfer of contaminants in the fluid due to the creation of large concentration gradients and by spreading the surface "contact" area between the host and contaminant fluids. Almost all fluids are turbulent, which is represented by a high Reynolds number $R=U d / \nu$ (where $U$ and $d$ are representative velocity and length scales, and $\nu$ is kinematic viscosity). Turbulent flow is characterized by unpredictable fluctuations in velocity, pressure, and concentration fields, and therefore a comparable approach is randomness in statistics described yet by [11]. This randomness of the turbulent flow as a whole is a result of random initial and boundary conditions, as well as random molecular motion. Such intrinsic randomness prevents one from using any deterministic theory to define the behavior of the concentration field in time and space. Thus, any application at least requires the point probability density function Eq. (1.2) to monitor the reduction of the concentration [74]. The importance and disadvantages of PDFs are described in more details in Section 2.2, whereas the focus is here on their standard and central moments which may later be used to reconstruct the PDFs themselves (Section 2.3).

Standard moments

$$
m_{n}(\mathbf{x}, t)=\overline{c^{n}(\mathbf{x}, t)}=\int_{0}^{\infty} c^{n}(\mathbf{x}, t) p(c ; \mathbf{x}, t) d c
$$

and central moments

$$
\mu_{n}(\mathbf{x}, t)=\overline{\left(c(\mathbf{x}, t)-m_{1}(\mathbf{x}, t)\right)^{n}}=\int_{0}^{\infty}\left(c(\mathbf{x}, t)-m_{1}(\mathbf{x}, t)\right)^{n} p(c ; \mathbf{x}, t) d c
$$

define the shape of the distribution and the density function. The main features of the concentration distribution are defined by lower order moments, specifically the first one (mean) defines the center of the PDF, the second one measures its width, while higher order moments mostly contribute to the tails of the PDF. In the absence of molecular diffusion, the expressions for the $\mathrm{n}$-th order central moments are known functions of the mean concentration:

$$
\begin{gathered}
\mu_{2}(\mathbf{x}, t)=\bar{c}\left(C_{0}-\bar{c}\right) \\
\mu_{3}(\mathbf{x}, t)=\bar{c}\left(C_{0}-\bar{c}\right)\left(C_{0}-2 \bar{c}\right) \\
\mu_{4}(\mathbf{x}, t)=\bar{c}\left(C_{0}-\bar{c}\right)\left(C_{0}^{2}-3 C_{0} \bar{c}+3 \bar{c}^{2}\right) \\
\mu_{5}(\mathbf{x}, t)=\bar{c}\left(C_{0}-\bar{c}\right)\left(C_{0}^{3}-4 C_{0}^{2} \bar{c}+6 C_{0} \bar{c}^{2}-4 \bar{c}^{3}\right) \\
\mu_{6}(\mathbf{x}, t)=\bar{c}\left(C_{0}-\bar{c}\right)\left(C_{0}^{4}-5 C_{0}^{3} \bar{c}+10 C_{0}^{2} \bar{c}^{2}-10 C_{0} \bar{c}^{3}+5 \bar{c}^{4}\right)
\end{gathered}
$$


or a more general expression given by [25]:

$$
\mu_{n}=\frac{\bar{c}}{C_{0}}\left(C_{0}-\bar{c}\right)^{n}+(-1)^{n}\left(1-\frac{\bar{c}}{C_{0}}\right) \bar{c}^{n}
$$

where $C_{0}$ is the source concentration, an overbar denotes an ensemble average, and the dependence on $(\mathbf{x}, t)$ is hereafter omitted for the sake of simplicity. Moreover, multiplying Eq. (1.1) by $c^{n}(\mathbf{x}, t)$ and taking the ensemble average, the concentration mass balance, in terms of generic standard moments $m_{n+1}$ [26], with detailed evaluation in Appendix A, can be written as:

$$
\frac{\partial m_{n+1}}{\partial t}+\nabla \cdot \overline{v c^{n+1}}=e_{m} \nabla^{2} m_{n+1}-n(n+1) e_{m} \overline{c^{n-1}(\nabla c)^{2}}
$$

The first term on the right side of Eq. (2.4) is a rather small diffusive term which is usually neglected when evaluating concentration moments due to its minor effect compared to the advective term $\nabla \cdot \overline{v c^{n+1}}$, yielding the equation for concentration moments:

$$
\frac{\partial m_{n+1}}{\partial t}+\nabla \cdot \overline{v c^{n+1}}=-n(n+1) e_{m} \overline{c^{n-1}(\nabla c)^{2}}
$$

It should be mentioned that, apart from the negligible term $\left(e_{m} \nabla^{2} m_{n+1}\right)$, the above equation exactly describes the time evolution of the concentration moments development. Advection by turbulent velocity and molecular diffusion are two fundamental physical processes responsible for the dispersion of a conservative solute. The Peclet number $P e=u \ell / e_{m}$, where $u$ and $\ell$ are appropriate velocity and length scales of turbulent fluctuations, quantifies the relative importance of advection and diffusion processes. In most environmental flows, the Peclet number is very large, indicating that turbulent advection acts on a much shorter timescale than molecular diffusion. The ratio of these timescales can be estimated by $P e^{-1}$ if the length scales for the velocity and concentration fields are comparable [105]. This approach was previously implemented in the development of concentration moments in contaminant clouds such as in the atmosphere [140] or in a groundwater medium [4,5]. However, it can also be applied to estimate concentration statistics in steady flows, such as those produced by contaminant jets, wakes, and plumes. Hence, it was demonstrated in the atmosphere $[120,121]$ and in a groundwater environment [39]. The concentration moments on a plane normal to the mean flow direction $x$ (see Fig. 1.2) are being analyzed. When applied to a steady source in a steady flow, (Eq. (2.5)) becomes:

$$
\bar{v} \cdot \nabla \overline{c^{n+1}}+\nabla \cdot \overline{v^{\prime} c^{n+1}}=-n(n+1) e_{m} \overline{c^{n-1}(\nabla c)^{2}}
$$


where $v=\bar{v}+v^{\prime}$, and $\overline{v^{\prime}}=0$ by definition of Reynolds averaging. The term $\nabla \cdot \overline{v^{\prime} c^{n+1}}$ represents advection by turbulent velocity and quickly acts to stretch the plume in thin sheets and strands $[11,16]$. Molecular diffusion is a much slower process, but nevertheless the only one responsible for limiting the smallest scales present in the concentration field.

Molecular diffusion (represented by the coefficient $e_{m}$ ) reduces the concentration slowly with respect to advection spreading, and thus the second term on the left-hand side of Eq. (2.6) is approximated with the expression resulting in zero molecular diffusion $\left(e_{m}=0\right)$. In this case, the exact standard concentration moment reads $m_{n+1}=C_{0}^{n} \bar{c}$, where $C_{0}$ is the initial concentration at the river mouth. It is convenient to set $C_{0}=\bar{c}(0,0)$ as previously described by [139, 140], where $\bar{c}(\mathbf{x})=m_{1}(\mathbf{x})$ is the mean concentration. The second term of the left-hand side in Eq. (2.6)) is thus approximated as [140]:

$$
\nabla \cdot \overline{v^{\prime} c^{n+1}}=-\bar{v} \cdot \nabla\left(C_{0}^{n} \bar{c}\right)
$$

By introducing Eq. (2.7) into Eq. (2.6), the following equation for concentration moments is obtained:

$$
\bar{v} \cdot \nabla \overline{c^{n+1}}-\bar{v} \cdot \nabla\left(C_{0}^{n} \bar{c}\right)=-n(n+1) e_{m} \overline{c^{n-1}(\nabla c)^{2}}
$$

Turbulent characteristics of the flow are represented by advective action embodied by the term $\bar{v} \cdot \nabla\left(C_{0}^{n} \bar{c}\right)$, which controls pulling out of the contaminant into ever-thinning sheets and strands until the thinning is limited by thickening due to molecular diffusion. Eventually, these competing actions balance each other out, creating sheets and strands of a thickness comparable to the Batchelor conduction cut-off length, $\lambda$, ranging about $10^{-5}-10^{-3} \mathrm{~m}$ in most environmental flows [29]. The $\lambda$ length is also recognized as a contaminant microscale [23] and is specifically estimated at the order of $10^{-4} \mathrm{~m}$ in the ocean [27], which is applicable to rivers and estuaries. Significant values of $(\nabla c)^{2}$ are finally expected to occur only over those thin sheets and strands at an effective scale $\lambda$, so that the concentration gradient is approximated by [107]:

$$
\nabla c=\frac{c-c_{t}}{\lambda}
$$

where $c_{t}$ is a background threshold concentration such that $c_{t} \longrightarrow 0$ for $t \longrightarrow 0$ and $c_{t} \longrightarrow \bar{c}$ for $t \longrightarrow \infty$. It is always intriguing to define the threshold concentration of the background medium in order to obtain the most probable concentration gradient. Considering a compromise between the two specified limits and 


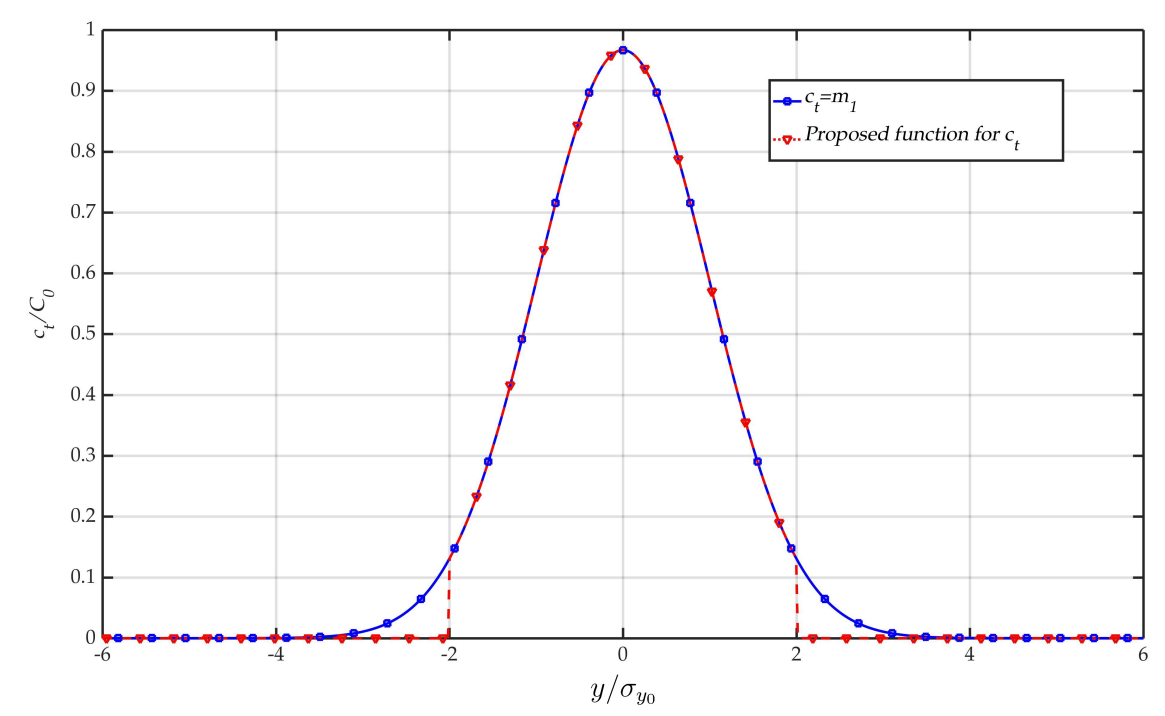

FIGURE 2.1: Proposed function for threshold concentration $c_{t}$.

that concentration moments are analyzed on a plane normal to the mean flow, an approximation for $c_{t}$ is proposed in the following form:

$$
c_{t}=\bar{c}\left(1-\frac{|y|}{2 \sigma_{y}}\right)\left[1-H\left(|y|-2 \sigma_{y}\right)\right]
$$

where $\sigma_{y}$ is the spatial concentration standard deviation and $H(y)$ is the Heaviside function. In this way, $c_{t}$ encompasses the near-field zone inside the perimeter of $2 \sigma_{y}$ such that $c_{t}=\bar{c}$ at $y=0$ and it linearly reduces to an area where it becomes zero after an expected distance of $2 \sigma_{y}$, as represented in Fig. 2.1.

In this research, the source characteristics consist of a river discharge which dominates the mixing process and thus the mean velocity vector is $\bar{v}(\mathbf{x}, t)=$ $U\left[U_{x}, 0,0\right]$, denoted as $U$ in further expressions.

The substitution of Eq. (2.9) in Eq. (2.8) yields the governing equation for the concentration standard moments for $n \geq 1$ :

$$
\frac{\partial m_{n+1}}{\partial x}+\frac{k \alpha}{U}\left(m_{n+1}-2 m_{n} c_{t}+c_{t}^{2} m_{n-1}\right)=C_{0}^{n} \frac{\partial \bar{c}}{\partial x}
$$

where $k=n(n+1), \alpha=e_{m} / \lambda^{2}$ and $\lambda$ is taken to be constant throughout the flow. Eq. (2.11) is a first-order linear ordinary differential equation and has a known solution, but depending on the form of the mean velocity $U$, it will be developed in the following subsections. 


\subsubsection{Concentration moments for the specific case of constant mean velocity}

When analyzing near-field mixing in an estuary, a special case of constant mean velocity may be considered. This situation occurs when the river discharge dominates the advection process in a shallow area near the river mouth. By following the general approach from [140] and applying the constant mean velocity $U$ in Eq. (2.11), a recursive solution for concentration moments is obtained:

$$
m_{n+1}(x)=e^{-\int_{0}^{x} \frac{k \alpha}{U} d \xi} \int_{0}^{x}\left\{\frac{k \alpha}{U}\left[2 m_{n}(\xi) c_{t}-m_{n-1}(\xi) c_{t}^{2}\right]+C_{0}^{n} \frac{\partial \bar{c}(\xi)}{\partial \xi}\right\} e^{\int^{\xi} \frac{k \alpha}{U} d \psi} d \xi
$$

In order to resolve the $\partial \bar{c} / \partial \xi$, the last part of the integral is then subjugated to a partial integration:

$$
\int_{0}^{x} C_{0}^{n} \frac{\partial \bar{c}}{\partial \xi} e^{\int^{\xi} \frac{k \alpha}{U} d \psi} d \xi=C_{0}^{n} \bar{c}(x) e^{\frac{k \alpha}{U} x}-\frac{k \alpha}{U} \int_{0}^{x} C_{0}^{n} \bar{c}(\xi) e^{\frac{k \alpha}{U} \xi} d \xi
$$

which enables the following transformation:

$$
\begin{aligned}
m_{n+1}(x) & =e^{-\frac{k \alpha}{U} x} \int_{0}^{x} \frac{k \alpha}{U}\left[2 m_{n}(\xi) c_{t}-m_{n-1}(\xi) c_{t}^{2}\right] e^{\int^{0} \frac{k \alpha}{U} d \psi} d \xi \\
& +e^{-\frac{k \alpha}{U} x}\left[C_{0}^{n} \bar{c}(x) e^{\frac{k \alpha}{U} x}-\frac{k \alpha}{U} \int_{0}^{x} C_{0}^{n} \bar{c}(\xi) e^{\frac{k \alpha}{U} \xi} d \xi\right]
\end{aligned}
$$

Finally, after all the evaluation steps, the recursive equation for concentration moments is obtained:

$$
m_{n+1}(x)=C_{0}^{n} \bar{c}(x)+\frac{k \alpha}{U} e^{-\frac{k \alpha}{U} x} \int_{0}^{x}\left[2 m_{n}(\xi) c_{t}-m_{n-1}(\xi) c_{t}^{2}-C_{0}^{n} \bar{c}(\xi)\right] e^{\frac{k \alpha}{U} \xi} d \xi
$$

The evaluation of the standard and central concentration moments for $n=1,2,3,4,5$ is given in Appendix B.1. 


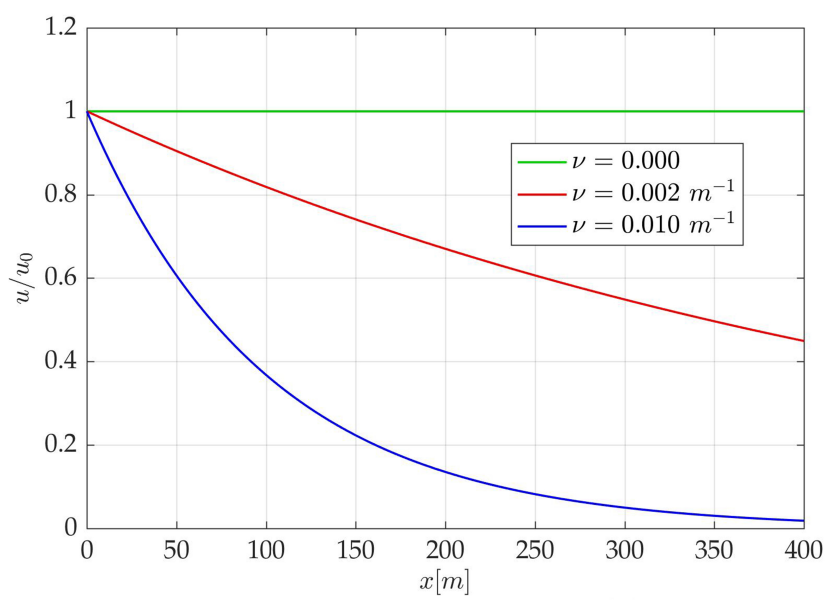

FIGURE 2.2: Impact of different attenuation coefficients $-\nu\left[\mathrm{m}^{-1}\right]-$ on the velocity field.

\subsubsection{Concentration moments for a non-uniform mean velocity}

Mixing in an estuary can be related to different sources, e.g. wind-induced currents, tide, river discharge, density gradients. In the near-field zone adjacent to the estuary mouth, the river discharge is the main mixing generator although the other sources could also have an influence. For example, the velocity under tidal modulation may be described as [80]:

$$
v(\mathbf{x}, t)=v_{0}+v_{\max T} \sin \sigma(t-\delta)
$$

where $v_{0}$ is riverine velocity, $v_{\max T}$ is the maximum value of tidal velocity, $t$ is the time measured from the beginning of injection of a pollutant, and $\delta$ is a constant representing the time shift between the injection time and the time of zero tidal velocity.

In many physical processes, observation leads to an empirical description followed by a physical argument for its validity [53]. Several measurements have been conducted at the Žrnovnica river as explained in Chapter 4 and, from those results, an approximation for the depth-integrated mean velocity $U$ in the nearfield area and a steady source is given:

$$
U(x)=U_{0} e^{-\nu x}
$$

where $U_{0}$ is the cross-sectionally averaged velocity at the river mouth and $\nu\left[\mathrm{m}^{-1}\right]$ is an attenuation coefficient representing all existing mechanisms that, when combined, concur to progressively reduce $U$ in the $x$ direction; including terrains geometry, bathymetry, tides, wind, sea currents, and salinity stratification. 
The idea to use this mean velocity model arises from the physical concept of a river entering a much larger static water body (lake or sea), which possesses enough inertia to systematically reduce the initial velocity as the flow propagates downstream. The influence of the velocity attenuation coefficient defined this way is shown in Fig. 2.2. When investigating velocity propagation downstream from the river mouth, as far as the near field and steady state are considered, the river velocity is dominant and therefore sea currents might have a rather limited impact. Note that in most cases wind waves potentially have a positive impact on the pollution concentration dynamic by enhancing additional dilution. However, for some estuary morphologies it may also contract the plume to a smaller area closer to the coast [40] by increasing the local solute mass.

As shown by $[80,53,45,83]$, the velocity and salinity profiles may become quite complex in the near-field zones of estuaries. The presence of increased horizontal density gradient stability on the ebb and reduced ones on the flood [130], can nevertheless be modeled by ebb reducing, and flood increasing the attenuation coefficient $\nu$. Recent research carried out by [151] has also shown that the onshore wind may influence mixing in the estuary by increasing surface turbulence, and change the velocity profile due to wind-enhanced plume shear, resulting in a decreased surface velocity. The reduced mean velocity, Eq. (2.17), might be applied when studying the worst-case scenario for the pollution transport, whereby the probability of having higher solute concentration in the coastal area adjacent to the estuary mouth is increased.

By introducing Eq. (2.17) into Eq. (2.11), the governing equation for concentration moments is developed into the form:

$$
\frac{\partial m_{n+1}}{\partial x}+\frac{k \alpha}{U_{0} e^{-\nu x}}\left(m_{n+1}-2 m_{n} c_{t}+c_{t}^{2} m_{n-1}\right)=C_{0}^{n} \frac{\partial \bar{c}}{\partial x}
$$

which has a solution for the presented linear ordinary differential similar to Eq. (2.12):

$$
\begin{aligned}
m_{n+1}(x) & =e^{-\int_{0}^{x} \frac{k \alpha}{U_{0} e^{-\nu \xi}} d \xi} \\
& \times \int_{0}^{x}\left\{\frac{k \alpha}{U_{0} e^{-\nu \xi}}\left[2 m_{n}(\xi) c_{t}-m_{n-1}(\xi) c_{t}^{2}\right]+C_{0}^{n} \frac{\partial \bar{c}(\xi)}{\partial \xi}\right\} e^{\int^{\xi} \frac{k \alpha}{U_{0} e^{-\nu \psi}} d \psi} d \xi
\end{aligned}
$$


Again, the last part of the integral is then subjugated to partial integration:

$$
\int_{0}^{x} C_{0}^{n} \frac{\partial \bar{c}(\xi)}{\partial \xi} e^{\int^{\xi} \frac{k \alpha}{U_{0} e^{-\nu \psi}} d \psi} d \xi=C_{0}^{n} \bar{c}(x) e^{\frac{k \alpha}{U_{0} \nu}\left(e^{-\nu x}-1\right)}-\frac{k \alpha}{U_{0} e^{-\nu x}} \int_{0}^{x} C_{0}^{n} \bar{c}(\xi) e^{\left[\frac{k \alpha}{U_{0} \nu}\left(e^{-\nu \xi}-1\right)+\nu \xi\right]} d \xi
$$

which enables:

$$
\begin{aligned}
m_{n+1}(x) & =e^{-\frac{k \alpha}{U_{0} e^{-\nu x}}\left(e^{\nu x}-1\right)} \int_{0}^{x} \frac{k \alpha}{U_{0}}\left[2 m_{n}(\xi) c_{t}-m_{n-1}(\xi) c_{t}^{2}\right] e^{\frac{k \alpha}{U_{0} \nu}\left(e^{-\nu \xi}-1\right)} e^{\nu \xi} d \xi \\
& +e^{-\frac{k \alpha}{U_{0} e^{-\nu x}}\left(e^{\nu x}-1\right)}\left[C_{0}^{n} \bar{c}(x) e^{\frac{k \alpha}{U_{0} \nu}\left(e^{-\nu x}-1\right)}-\frac{k \alpha}{U_{0} e^{-\nu x}} \int_{0}^{x} C_{0}^{n} \bar{c}(\xi) e^{\left[\frac{k \alpha}{U_{0} \nu}\left(e^{-\nu \xi}-1\right)+\nu \xi\right]} d \xi\right]
\end{aligned}
$$

Finally, a recursive equation for concentration moments with a non-uniform mean velocity field is obtained:

$$
\begin{aligned}
m_{n+1}(x) & =C_{0}^{n} \bar{c}(x)+\frac{k \alpha}{U_{0}} e^{-\frac{k \alpha}{U_{0} \nu}\left(e^{\nu x}-1\right)} \\
& \times \int_{0}^{x}\left[2 m_{n}(\xi) c_{t}-m_{n-1}(\xi) c_{t}^{2}-C_{0}^{n} \bar{c}(\xi)\right] e^{\left[\frac{k \alpha}{U_{0} \nu}\left(e^{\nu \xi}-1\right)+\nu \xi\right]} d \xi
\end{aligned}
$$

The derivation of standard and central concentration moments for $n=1,2,3,4,5$ for the case of a non-uniform mean velocity field is given in Appendix B.2. Fig. 2.3 shows several examples of calculated central moments for the case of attenuated velocity field by $\nu=0.002 \mathrm{~m}^{-1}$. The results are given in more detail in Chapter 5 .

\subsection{Probability density function for pollution concen- tration}

A general issue of coastal water quality risk assessment is that it requires the information on the probability of exceeding the allowed concentration values, as defined by environmental quality standards (e.g. [48]). In order to obtain the probability of exceeding these limit values, it is necessary to have an estimate of the concentration probability density function (PDF) or its cumulative counterpart - cumulative density function (CDF). This representation can be used to obtain information on the probability of exceeding the allowed concentration values in the recipient medium. 

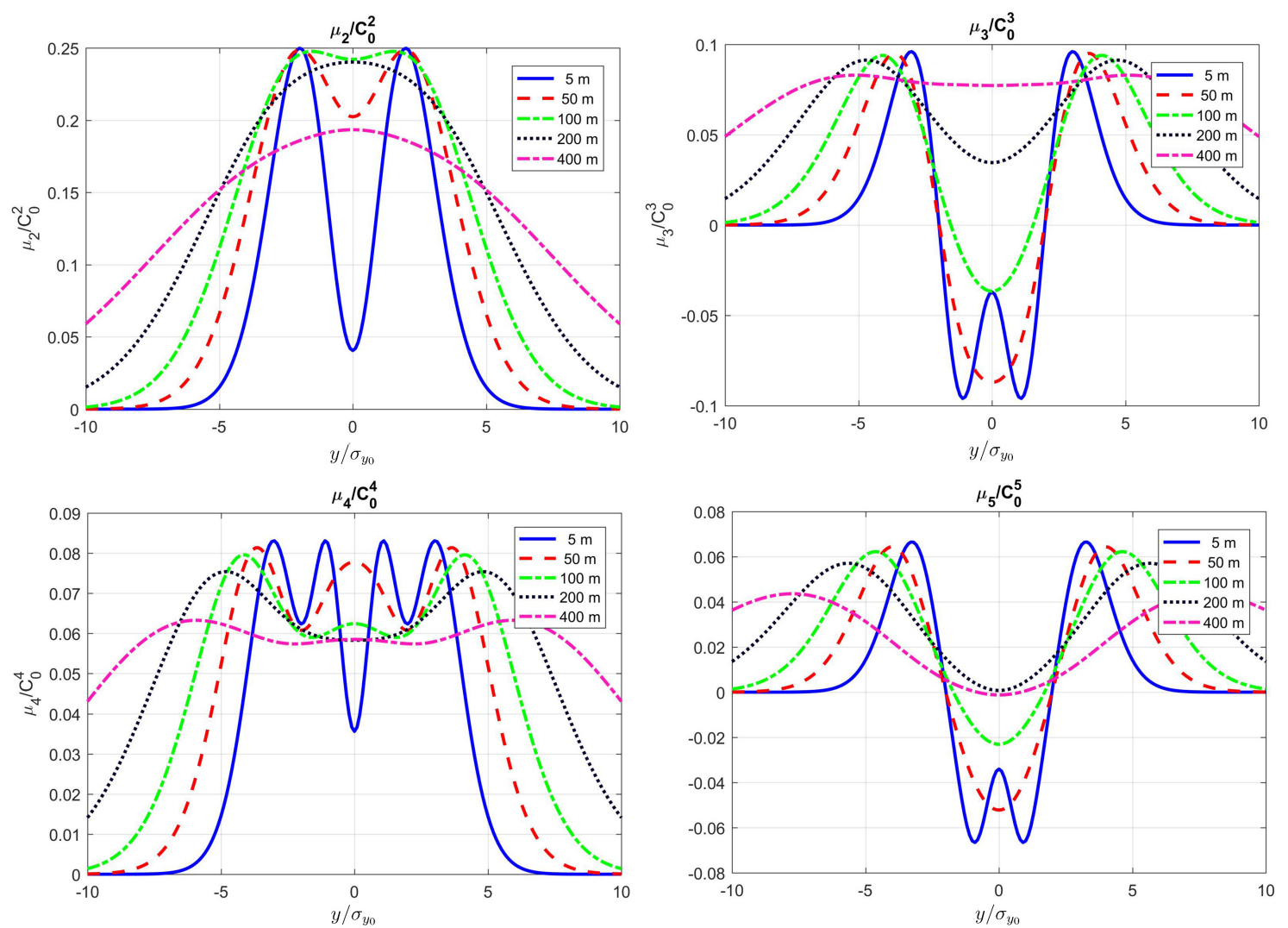

FIGURE 2.3: Examples of concentration standard moments: $\mu_{2}, \mu_{3}$, $\mu_{4}$ and $\mu_{5}$ at different distances from the source.

The evolution of a contaminant concentration field in a turbulent flow has an intrinsically random nature due to the mixing dynamics which take place on very small length scales [125]. Such random processes are usually represented by the PDF defined by Eq. (1.2) in its general form, where the value of concentration is bounded,

$$
0 \leq c \leq C_{0}
$$

Concentration is therefore a continuous random variable in the region $\left[0, C_{0}\right]$ such that the CDF is defined:

$$
P(\mathbf{x}, t)=\int_{0}^{C_{0}} p(c ; \mathbf{x}, t) d c=1
$$

where $p(c ; \mathbf{x}, t)$ is the PDF defined in $(\mathbf{x}, t)$ such that it always applies $p(c ; \mathbf{x}, t) \geq$ 0 . Eq. (2.24) also meets the fundamental requirement of the PDF by having the 
overall integral equal to 1, while the integral limits are defined by Eq. (2.23) instead of a general $[-\infty,+\infty]$. Following the Eq. (2.24), the probability of exceeding, $P_{E}\left(c^{*}\right)$, for an arbitrary concentration, $c^{*}$, can be defined as:

$$
P_{E}\left(c^{*}\right)=\int_{c^{*}}^{C_{0}} p(c ; \mathbf{x}, t) d c
$$

This application of the PDFs and CDFs is further described and discussed within results in Chapter 5 and particularly in Chapter 6 as a final practical application of the developed methodology.

As previously discussed, the physical problem being looked into by this thesis is the mixing process represented by the advective term, which acts on a relatively fast timescale, and the diffusive term, which is the only process responsible for concentration reduction, i.e. with values between 0 and $C_{0}$. For a hypothetical case of no molecular diffusion $\left(e_{m}=0\right)$, as described by [25, 105], the contaminant is pulled into ever-thinning sheets and strands by advection, but they always remain at the initial source concentration $\left(C_{0}\right)$. That behavior is represented by a two-state distribution (e.g. $[85,13])$ with the existence of only two elements in the sample space. The PDF for such a distribution is a convex combination of Dirac delta functions:

$$
p(c ; \mathbf{x}, t)=(1-\pi) \delta(c)+\pi \delta\left(c-C_{0}\right)
$$

where $\pi$ represents the probability that a fluid at certain $(\mathbf{x}, t)$ is coming from the source and it is called the intermittency [28]. Despite being slower, the diffusion process does occur in reality, and it serves to reduce the concentration of sheetand strand-like structure developed by advection, ultimately increasing the concentration in the host fluid. The obvious disparity of the timescales at which the advective and the diffusive parts of ADE take place is the basis for approximation in Eq. (2.7). However, a slow but existent effect of molecular diffusion is visible through gradual growth of the intermittency part of the PDF as one attains the downstream PDF, while the PDF close to the source is very similar to the double Dirac function in Eq. (2.26). Fig. 2.4 demonstrates this delayed but important effect of molecular diffusion on the pollution concentration field. Other examples of PDF and CDF results are provided with the rest of the results in Chapter 5. In conclusion, the PDF or the CFD as a tool provides very important input data on the pollution load at a certain water body needed for a more detailed risk analysis. Incidentally, it enables a quick estimate of expected pollution loading at the specific location, which is why it forms part of the screening tool developed within the CPoRT application (see Chapter 6). 

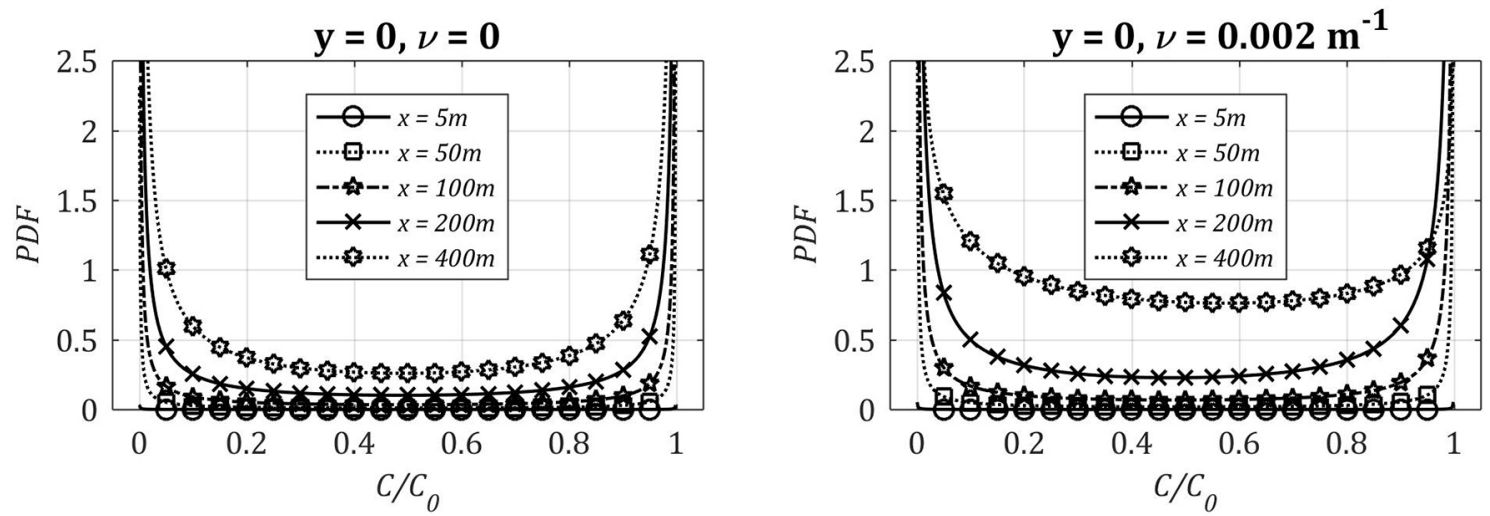

FIGURE 2.4: Concentration beta distribution PDFs for 5 different distances from the source $(x=5,50,100,200,400 \mathrm{~m})$, at centerline for attenuation coefficient $\nu=0$ (left) and for attenuation coefficient $\nu=0.002 \mathrm{~m}^{-1}$ (right).

Some moment inversion techniques are briefly examined below.

\subsection{Moment inversion to obtain the PDF}

In order to obtain the approximation of the pollution concentration PDF, some of the lower-order moments may be inverted, since there are compelling practical reasons for that, as previously discussed $[142,41]$ and it will be applied within the CPoRT software in Chapter 6. Among different inversion methods, presented here are the moment-matching fit using theoretical distribution (Beta) and the maximum entropy method (MaxEnt).

\subsubsection{Moment-matching method}

This process allows choosing between different theoretical distributions in such a way that analytically obtained or measured moments are equal to the moments of the selected theoretical distribution. The distribution shape parameters are calculated from the known moments to define the theoretical functions. When choosing the most likely theoretical distribution, it is advisable to take into account the behavior of the random process that is being described. The PDF for contaminant concentration reduction in turbulent flow has constraints $\left[0, C_{0}\right]$ or in a normalized case of $[0,1]$, and it has a two-state nature with a tendency to obtain a U-shape distribution (see Fig. 2.4). All of these characteristics make the Beta distribution an adequate choice since it has previously been used in other environmental flows $[24,128]$ and the U-shape distribution is an example of a 
Beta distribution [21]. Beta distribution is defined by:

$$
f(c)=\frac{c^{\alpha-1}(1-c)^{\beta-1}}{B(\alpha, \beta)}
$$

where $\alpha, \beta$ are the shape parameters of distribution, and the Beta function is:

$$
B(\alpha, \beta)=\int_{0}^{1} c^{\alpha-1}(1-c)^{\beta-1} d c
$$

Beta distribution moments are obtained using a recursive prescription:

$$
m_{n}=\frac{\alpha+n-1}{\alpha+\beta+n-1} m_{n-1}
$$

where $\alpha$ and $\beta$, when related to the previously developed moments, are defined by:

$$
\begin{gathered}
m_{1}=E(x)=\frac{\alpha}{\alpha+\beta} \\
\mu_{2}=\operatorname{Var}(x)=\frac{\alpha \beta}{(\alpha+\beta)^{2}(\alpha+\beta+1)}
\end{gathered}
$$

By using previously obtained moments $\left(m_{1}\right.$ and $\left.\mu_{2}\right)$ in Section 2.1 , the distribution parameters are calculated:

$$
\begin{gathered}
\alpha=\frac{m_{1}^{2}\left(1-m_{1}\right)}{\mu_{2}}-m_{1} \\
\beta=\frac{\alpha\left(1-m_{1}\right)}{m_{1}}
\end{gathered}
$$

This approach is subsequently used to obtain the PDFs at any point of the available domain (see examples in Fig. 2.4), which are then presented in Chapter 5 .

\subsubsection{Maximum entropy method - MaxEnt}

The moment-matching method is a very useful approach to the solution of transport equations for PDFs [94]. This procedure, however, smooths out much of the information contained in the continuous distribution and it is sometimes desirable to calculate the continuous distribution which is unbiased by the chosen theoretical distributions. The maximum entropy method provides a means of reconstructing the PDF given a finite number of moments. This is commonly known as 
the classic moments problem. In general, MaxEnt, as named by [84], has the purpose of finding a continuous distribution whose moments match the known moments while obtaining the least-biased estimate. Such a PDF presents a robust tool for prediction, but also one with the highest uncertainty as it ignores all unknown information [69].

The MaxEnt method is based on the concept that the distribution which maximizes the information entropy is the one that is statistically the most likely to occur. In the context of information theory by [129], the information entropy $S$, of a distribution $p(c)$, is given by the integral:

$$
S(p)=-\int_{\Omega} \ln [p(c)] p(c) d c
$$

where $\Omega$ is the support of the distribution, but, for the physical problem of concentration distribution observed here, the domain is defined in the form of $\left[0, C_{0}\right]$ and it is hereafter used as such. The purpose, as explained above, is to find the $p(c)$ that maximizes the entropy function $S(p)$ [84], but in accordance with the constraints, i.e. known moments. The problem is then formulated as a variational one:

$$
\max S[p(c)]
$$

$$
\int_{0}^{C_{0}} c^{k} p(c) d c=m_{k} ; \quad k=0, \ldots, N
$$

where $(N+1)$ is the number of known moments. The optimization problem is then solved by introducing the Lagrangian function and the corresponding multipliers $\lambda_{k}[14,147]$, which define a functional [37]:

$$
\begin{aligned}
H & =S+\sum_{k=0}^{N} \lambda_{k}\left(\int_{0}^{C_{0}} c^{k} p(c) d c-m_{k}\right) \\
& =-\int_{0}^{C_{0}} \ln [p(c)] p(c) d c+\sum_{k=0}^{N} \lambda_{k}\left(\int_{0}^{C_{0}} c^{k} p(c) d c-m_{k}\right)
\end{aligned}
$$

This functional has a maximum when: 


$$
\begin{gathered}
\frac{\partial H}{\partial \lambda_{k}}=0 \\
\frac{\partial H}{\partial p(c)}=0
\end{gathered}
$$

Eq. (2.36) returns the constraints, while Eq. (2.37) leads to:

$$
\frac{\partial H}{\partial p(c)}=-\int_{0}^{C_{0}}\{\ln [p(c)]+1\} d c+\sum_{k=0}^{N} \lambda_{k} \int_{0}^{C_{0}} c^{k} d c=0
$$

The dependence on $(c)$ is further omitted for the sake of simplicity. The first part of that derivative is:

$$
\frac{\partial}{\partial p} \int_{0}^{C_{0}} \ln (p) p d c=\int_{0}^{C_{0}} \frac{\partial}{\partial p}[\ln (p) p] d c=\int_{0}^{C_{0}}[1+\ln (p)] d c
$$

And the second part is obtained by deriving the function within the integral:

$$
\frac{\partial}{\partial p} \sum_{k=0}^{N} \lambda_{k}\left(\int_{0}^{C_{0}} c^{k} p d c-m_{k}\right)=\sum_{k=0}^{N} \lambda_{k}\left(\frac{\partial}{\partial p} \int_{0}^{C_{0}} c^{k} p d c_{k}\right)=\sum_{k=0}^{N} \lambda_{k} \int_{0}^{C_{0}} c^{k} d c_{k}
$$

Eq. (2.38) finally develops the shape of $p$ (the distribution), which will be the least biased one, as defined by MaxEnt conditions:

$$
\begin{aligned}
-\int_{0}^{C_{0}}[\ln (p)+1] d c+\int_{0}^{C_{0}} \sum_{k=0}^{N} \lambda_{k} c^{k} d c & =0 \\
\ln (p) & =\sum_{k=0}^{N} \lambda_{k} c^{k}-1 \\
p(c) & =e^{\sum_{k=0}^{N} \lambda_{k} c^{k}}
\end{aligned}
$$

where $\lambda_{0}^{*}=\lambda_{0}-1$. 


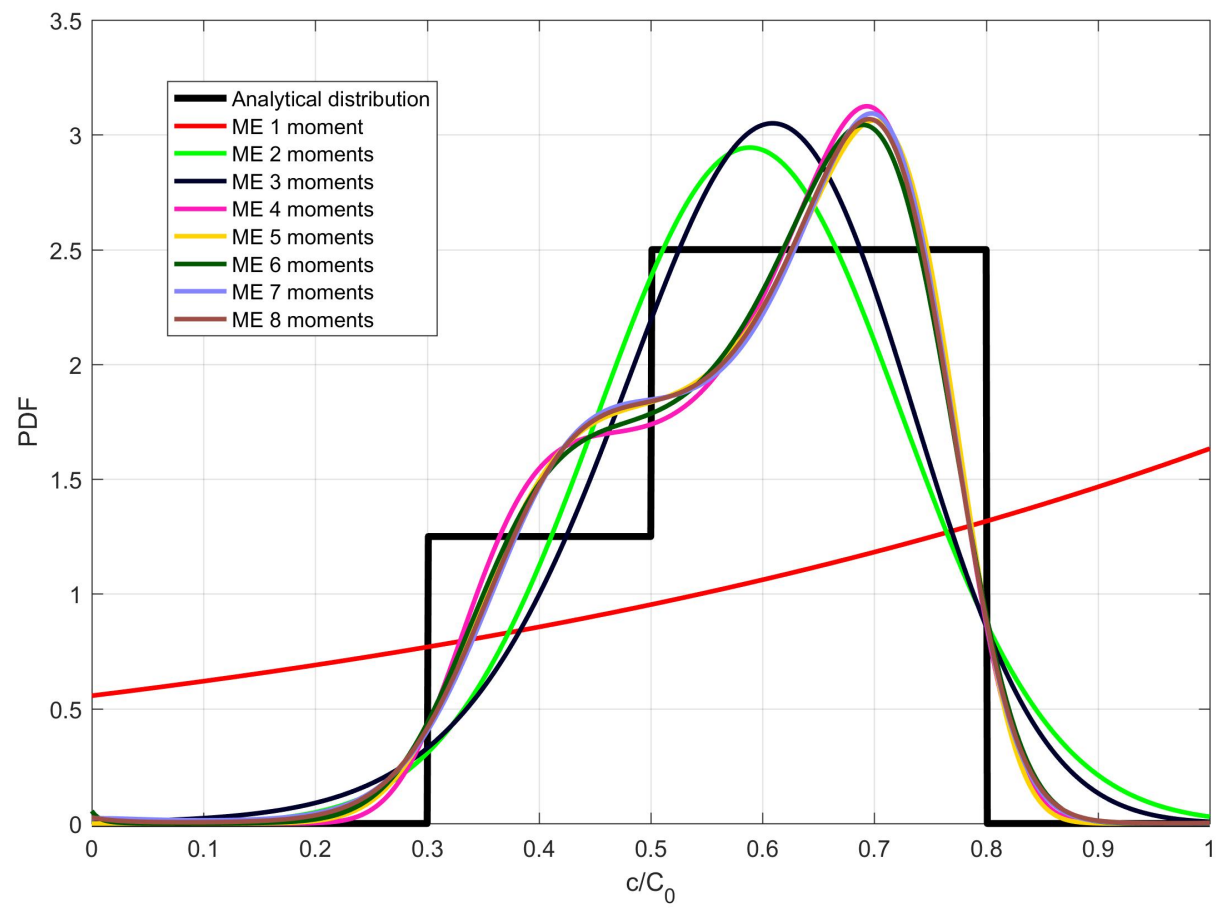

FIGURE 2.5: An example of the MaxEnt distribution estimate for the case of step function; the effect of using a higher number of moments.

The solution of the system is to be found by solving the next $(k+1)$ set of nonlinear equations:

$$
\begin{aligned}
& \int_{0}^{C_{0}} e^{\lambda_{0}+\lambda_{1} c+\ldots \lambda_{k} c^{k}} d c=m_{0} \\
& \int_{0}^{C_{0}} c e^{\lambda_{0}+\lambda_{1} c+\ldots \lambda_{k} c^{k}} d c=m_{1} \\
& \int_{0}^{C_{0}} c^{k} e^{\lambda_{0}+\lambda_{1} c+\ldots \lambda_{k} c^{k}} d c=m_{k}
\end{aligned}
$$

The system defined that way is subsequently solved by the trusted-region algorithm [100]. System integration is performed using the Gauss-Legendre quadrature (Eq. (2.43)): 


$$
\int_{0}^{C_{0}} c^{k} e^{\lambda_{0}+\lambda_{1} c+\ldots \lambda_{k} c^{k}} d c-m_{k} \approx \sum_{j=1}^{N} \omega_{j} c_{j}^{k} \cdot e^{\lambda_{0}+\lambda_{1} c+\ldots \lambda_{k} c^{k}}-m_{k}
$$

where $c_{j}$ are Legendre nodes. For the MaxEnt calculation in this thesis, 1000 nodes were used, remapped from the classical $[-1,1]$ to $[a, b]$ where $[a, b]=\left[0, C_{0}\right]$ :

$$
\int_{a}^{b} f(x) d x \approx \frac{b-a}{2} \sum_{i=1}^{n} w_{i} f\left(\frac{b-a}{2} x_{i}+\frac{a+b}{2}\right)
$$

Chosen initial values of $\lambda$ are:

$$
\lambda_{0}=-0.5, \lambda_{k}=0 ; \quad k=1, \ldots N
$$

Whilst multiple MaxEnt algorithms are developed ([101, 131, 129]) and they present the least biased and robust methods, many of them are constrained by the number of moments they can deal with.

A higher number of moments in general increases the accuracy of the estimate as it can be seen in Fig. 2.5. However, to reach that level of accuracy, one needs to tackle the issue of instable numerical calculation where the optimization issue becomes ill-conditioned [9]. Another issue that occurs is the computational time. As described in Fig. 2.6, by adding additional moments, the corresponding calculation time (blue dots in the figure) for each added moment increases exponentially in relation to the time needed for two moments.

To demonstrate the MaxEnt method, Fig. 2.7 shows PDFs obtained with MaxEnt using a different number of moments and compared to the corresponding Beta distribution for all the same parameters and with constant mean velocity $(\nu=0)$. The moments are obtained for constant mean velocity $(U=0.4 \mathrm{~m} / \mathrm{s})$ by a recursive equation (Eq. (2.15)) with the source width $b=10 \mathrm{~m}$ and unit steady mass loading. 


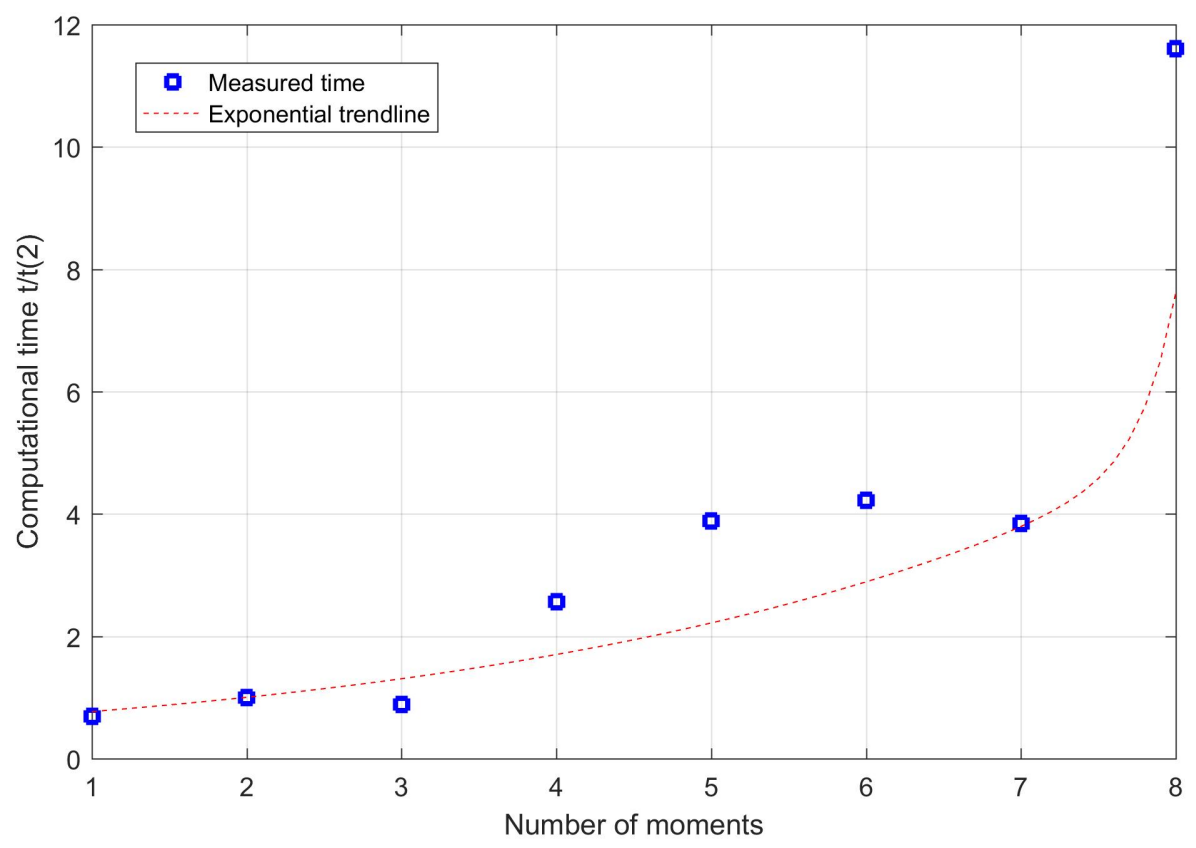

FIGURE 2.6: Computational time for the MaxEnt algorithm for a different number of used moments, normalized with two moments' calculation time.

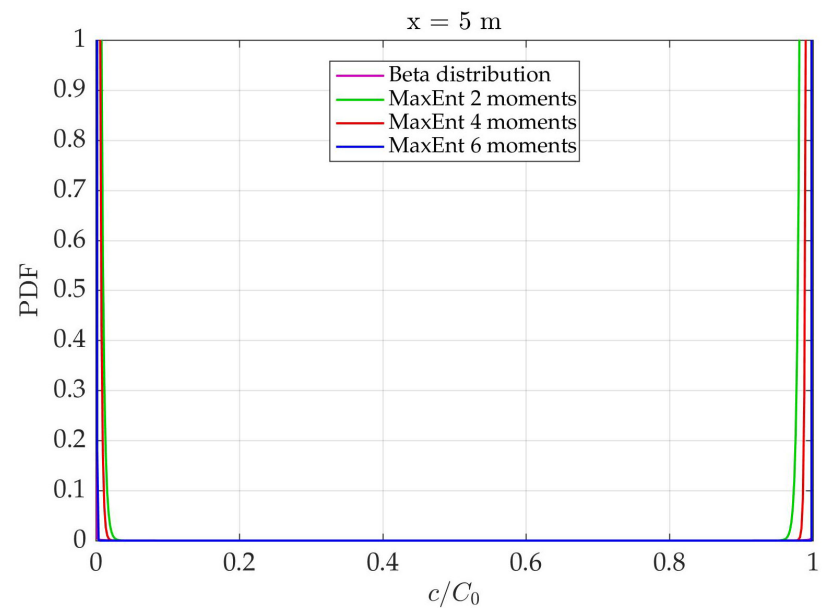

FIGURE 2.7: The concentration PDF at $x=5 \mathrm{~m}, y=0 \mathrm{~m}$, obtained by the MaxEnt method using a different number of moments $(n=$ $2,4,6)$ and compared to the corresponding Beta distribution.

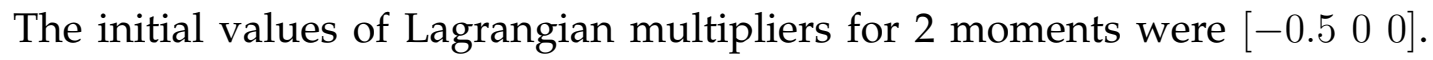
The calculation time and accuracy were slightly improved by using multipliers obtained in the 2 moments' case as the initial values of multipliers in the 4 moments' case, and so on for the 6 moments' case. Nevertheless, the calculation still took over half an hour per one point PDF. As it is demonstrated in the above 
figure, the PDF obtained by MaxEnt approaches the Beta distribution by increasing the number of moments. However, due to all of the aforementioned reasons, only the moment-matching method is further used to obtain PDFs and EMFs from their moments.

In conclusion, both the moment-matching and the MaxEnt algorithm represent potentially useful methods for obtaining PDFs from moments. MaxEnt is the least biased method, but it is burdened with several issues mentioned above, including numerical instability and high computational time. On the other hand, Beta distribution has been proven as a good assumption in multiple physical processes, and using it in the moment-matching method enables quick computation. Moment matching gives a more biased estimate but, when compared to measurements (in Chapter 5), they show an adequate overlap. These properties of moment matching, particularly quick calculation, are among the main reasons for its further use in this thesis and finally its implementation in the CPoRT computer application (Chapter 6). 



\section{Chapter 3}

\section{Spatially integrated concentration statistics}

This chapter is focused on a potential upgrade for describing the contaminant field dilution process in estuaries, using the concept of expected mass fraction EMF.

A part of methodology and scientific contribution of this chapter, with some additional development, is presented in the following paper which is currently under revision:

- Andricevic R., Galesic M. (2018). Dilution measure for the solute transport in an estuary, Advances in Water Resources.

The chapter consists of three sections. Section 3.1 lays out the background and foundation of the EMF concept (Section 3.1.1), while Section 3.1.2 applies its specific properties to the steady source of conservative contaminant in riverdominated estuaries. In Section 3.2, a connection is made between point statistics developed in Chapter 2 and EMF by applying direct integration to obtain the EMF. A different approach is applied in Section 3.3 by evaluating the integrated concentration moments $\left(K_{n}\right)$, which are subsequently used to obtain the EMF (similar to obtaining the PDFs from point moments in Section 2.3).

\subsection{Expected mass fraction - EMF}

When analyzing the pollution risk and the dilution measurement of a contaminant field in an environmental flow, obtaining the experimental statistical data is a very expensive and tedious process, whether they are laboratory or field measurements [133].

As already discussed in Chapter 2, environmental turbulent flows are intrinsically, to some extent, unsteady and spatially inhomogeneous, which causes variables such as velocity and scalar concentration to be random. However, 
the reduction of concentration values takes place only through molecular diffusion at contaminant microscale $(\lambda)$. It would be ideal to investigate the probability density function (PDF) of concentration as it evolves over time at each location in space to quantify the concentration field. However, the PDF is theoretically difficult since the equations that govern its evolution are intractable in even the simplest turbulent flows and it is challenging to measure. In the last two decades, many experimental possibilities have been developed and different measurements conducted with regard to hydrodynamic and pollution properties in rivers and estuaries $([65,89,153])$ due to technological developments. However, it is still a very expensive and tedious work [70], often followed by insufficient data when performing stochastic analysis and predictions, since those require a great number of realizations in any environmental flow $[23,13,72]$.

\subsubsection{EMF background}

In order to address this issue, authors have previously [26, 141] introduced the concept of cloud-averaged statistics which illustrated the advantage of using concentration statistics throughout the entire contaminant cloud in each realization, which would enable faster convergence of statistics. It was further developed as a less experimentally demanding measure of dilution [74, 75] and called the expected mass fraction [76, 143]. This concept was later used by several authors for further description of contaminant concentration field in the atmosphere [133, 126], and for exposure risk assessment of toxic chemicals dosage transported in aquifers $[6,136]$.

Generally, the EMF - $f(c ; t)$ is defined as:

$$
f(c ; t)=\frac{1}{M} \int_{V} c p(c ; \mathbf{x}, t) d V
$$

where $V$ denotes all space, $M$ is the conserved mass, $\int_{V} c(x, t) d v$, and $p$ is the corresponding point PDF. Hence, the expected fraction of total release mass $M$ between concentration $c_{1}$ and $c_{2}$ is:

$$
\operatorname{EMF}\left(c_{1}, c_{2}\right)=\int_{c_{1}}^{c_{2}} f(c ; t) d c
$$

For each cloud release in numerical or field tests, a mass fraction can be determined as follows:

$$
f^{\prime}\left(c^{\prime} ; t\right)=\frac{1}{M} \int_{V} c(\mathbf{x}, t) \delta\left(c(\mathbf{x}, t)-c^{\prime}\right) d V
$$




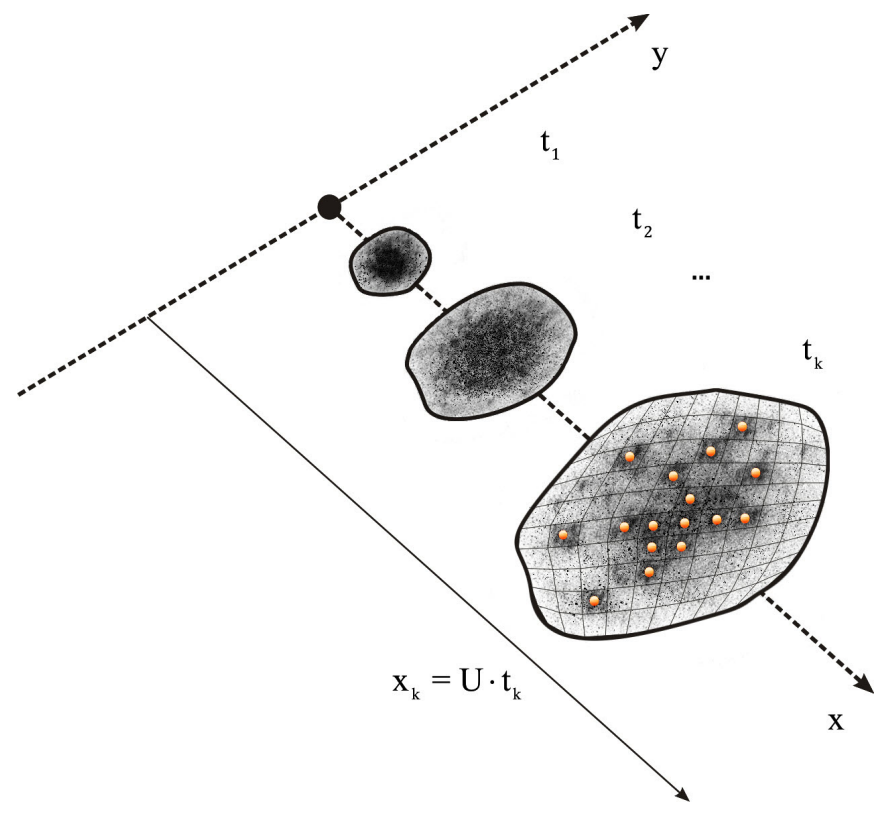

FIGURE 3.1: Schematic representation of the EMF concept for an arbitrary realization of contaminant cloud release.

such that ensemble average of $f^{\prime}\left(c^{\prime} ; t\right)$ equals $f(c ; t)$. The schematic representation of an arbitrary realization of cloud release is shown in Fig. 3.1. The mass fraction defined by Eq. (3.3) in one realization at $t_{k}$ can simply be obtained by counting $\left(N\left(\left|c-c^{\prime}\right| \leq \frac{\Delta c}{2}\right)\right)$ the cells containing the defined interval of concentration (the cells denoted by orange dots in Fig. 3.1). Hence, using the Iverson bracket for counting [82], the discrete form of Eq. (3.3) would be:

$$
f^{\prime}\left(c^{\prime} ; t\right)=\frac{\sum_{i=1}^{n}\left[c^{\prime} \in\left[c^{\prime}, c^{\prime}+d c^{\prime}\right]\right] \cdot c^{\prime} \cdot \Delta V}{M}
$$

Variations of $f^{\prime}\left(c^{\prime} ; t\right)$ about $f(c ; t)$ are expected to be reasonably small since, in each realization, concentration values throughout the entire cloud are used in the compilation of $f^{\prime}\left(c^{\prime} ; t\right)$ [154]. Furthermore, each mass interval $c(\mathbf{x}, t) d V(\mathbf{x})$ was found by summing up all of the volume elements $d v(\mathbf{x}, t)$ of the cloud with the concentration within a given interval $(\Delta c)$. As such, the defined sum of the mass in all intervals is equal to the release mass $M$, and by repeating that procedure for the release of many clouds, an average mass can be found, which then divided by the concentration interval defines a density function [154]. Furthermore, if intervals $(\Delta c)$ are made infinitesimally small and the integral is normalized by $M$, it is clear how the EMF resembles the PDF. Hence, the EMF has the same 
properties as the PDF, which may be easily demonstrated:

$$
\int_{0}^{\infty} f(c ; t) d c=\frac{1}{M} \int_{V} \int_{0}^{\infty} c p(c ; \mathbf{x}, t) d c d V=\frac{1}{M} \int_{V} \bar{c}(\mathbf{x}, t) d V=\frac{M}{M}=1
$$

Where $m_{n}=\int_{0}^{\infty} c^{n} p(c ; \mathbf{x}, t) d c$ are absolute moments of the point concentration PDF. The $f(c ; t)$ can account for any particular flow conditions (e.g. buoyancy effects, unsteadiness, or irregular boundaries) or the type of scalar contaminant. The only requirement is that the release mass $M$ be conserved. In a case where the release mass is not conserved, for example, where continuous release or chemical reactions are present, one would simply replace $M$ with $M(t)$ [133].

The corresponding cumulative distribution function $F(c ; t)$ is used to define the fraction of mass consisting of concentrations equal or higher than the limit value $c^{*}:$

$$
F\left(c^{*} ; t\right)=\int_{c^{*}}^{\infty} f(c ; t) d c
$$

The function $F\left(c^{*} ; t\right)$ has intuitive application to defining the loading of contaminant, i.e. the mass of contaminant which has the concentration above the $c^{*}$, in the observed and/or available volume, often defined as all space. That interpretation is very important in the environmental flow, where pollution is discharged into the environment (e.g. air pollution or discharge into the coastal zone and estuaries). Since the EMF has the same properties as the PDF, one may define its moments as follows:

$$
\begin{aligned}
& \widetilde{m_{n}}(t)=\int_{0}^{\infty} c^{n} f(c ; t) d c=\frac{1}{M} \int_{0}^{\infty} c^{n} \int_{V} c p(c ; \mathbf{x}, t) d V d c=\frac{1}{M} \int_{V} \int_{0}^{\infty} c^{n+1} p(c ; \mathbf{x}, t) d c d V \\
& \widetilde{m_{n}}(t)=\frac{K_{n+1}(t)}{M}
\end{aligned}
$$

where

$$
K_{n+1}(t)=\int_{V} m_{n+1}(\mathbf{x}, t) d V
$$

represents spatially integrated concentration moments and will be further discussed in Section 3.3. Evidently, EMF moments are related to specifically one order higher spatially integrated concentration moments $\left(K_{n+1}\right)$. 


\subsubsection{EMF for the case of contaminant plume with continuous line source}

In this thesis, the EMF concept follows a similar application to the one performed by [120] for the problems of contaminant plume in atmosphere; however, it is for the first time introduced as a potential dilution measure for concentration field reduction in surface waters, more precisely in estuaries. As the focus of this thesis is a river-dominated estuary with a steady, uniform mean flow velocity $U$ in the stream-wise direction $x$ and with the across plume direction $y$ (as described in Fig. 1.2), a modified definition of the EMF is introduced:

$$
\tilde{f}(c ; x)=\tilde{M}^{-1} \int_{-\infty}^{\infty} c p(c ; x, t) d y
$$

with $\tilde{M}=\int_{-\infty}^{\infty} \bar{c}(x, y) d y$. The Gaussian one-dimensional distribution is assumed for cross-sectional profiles commonly considered for wakes, jets, and plumes [53][4][124][140], and was also used for the river discharges to the estuary and the coastal zone [58]:

$$
\bar{c}(y, x)=\frac{L}{\sigma_{y} \sqrt{2 \pi}} e^{\frac{-y^{2}}{2 \sigma_{y}^{2}}}
$$

where $L=\frac{\dot{m}}{b U_{0}}$ is a steady mass loading of the solute through the plane normal to the flow direction $x$, defined by steady mass flux $(\dot{m})$, river mouth width $(b)$ and the initial velocity $\left(U_{0}\right)$. The spatial variance is defined by $\sigma_{y}^{2}=2 e_{t} t+\sigma_{y_{0}}^{2}, x=$ $U t$, where $e_{t}$ is the constant spatial variance growth rate (equivalent to turbulent diffusivity) across the flow in the direction $y$ [140], and $\sigma_{y_{0}}^{2}$ represents the initial variance at $x=0$.

To represent a comparison between cloud and plume cases of contaminant release in the EMF concept, an arbitrary realization of contaminant plume release is shown in Fig. 3.2. The concept of mass fraction defined by Eq. (3.3) and Eq. (3.4) is equivalent in the plume case too, except for the definition of mass, which is in this case represented by a steady mass loading $(L)$. The discrete form of mass fraction for an arbitrary plume realization at a chosen slice $\left(x_{k}\right)$ defined by $t=x_{k} / U$ is therefore defined by an equivalent form of Eq. (3.4), by changing $M$ to $L$. All cells exhibiting concentration in the interval defined by $\left(\left|c-c^{\prime}\right| \leq \frac{\Delta c}{2}\right)$ are counted for (orange dots in Fig. 3.2), and the mass of contaminant at each cell is defined by $c^{\prime} \cdot \Delta V$. Therefore, modified Eq. (3.9) defines the mass fraction of the steady mass loading $(L)$ at the cross section $x_{k}$.

The following two sections present different ways of obtaining the EMFs, with an emphasis on the problem of contaminant plume release. 


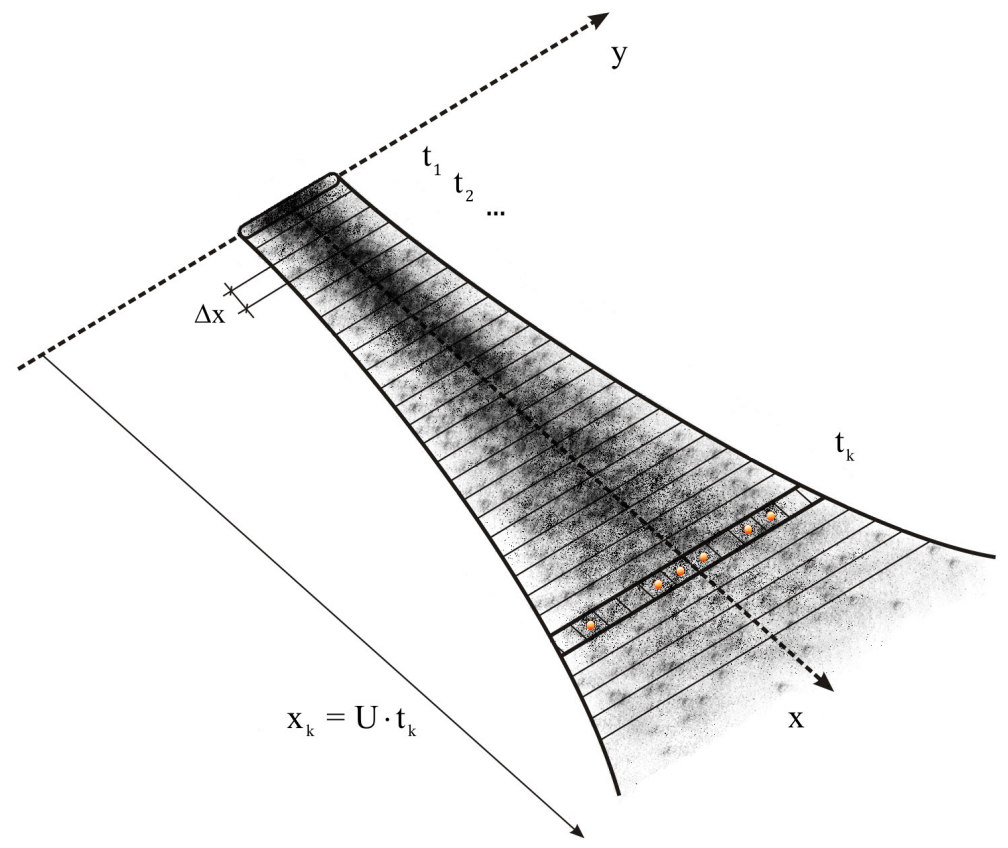

FIGURE 3.2: Schematic representation of EMF concept for an arbitrary realization of contaminant plume release.

\subsection{Direct integration of PDFs}

Point statistics give adequate description of contaminant concentration reduction throughout the space and time domains; however, here they are upgraded to integrated statistics where all space is represented by the perpendicular slice of the downstream estuary (as described in Section 3.1.2). Hence, the approach follows the problem formulation from Section 1.1 represented visually by Fig. 1.2, and now the results from Chapter 2 are used in the form of obtained PDFs. The procedure is a straightforward application of the EMF definition by Eq. (3.1), where a numerical integration is applied to calculate the discrete representation of the EMF [55]:

$$
f_{i}\left(c ; x_{i}\right)=\frac{1}{L} \sum_{c} \sum_{y} c p\left(c ; x_{i}, y\right) \Delta c
$$

where $i$ represents each downstream slice with the thickness defined as $d x$. Therefore, the volume from Eq. (3.1) represents the available volume considered to be conserved and the constant mass input $L$ due to the continuous steady state being observed (Section 3.1.2). Following the Eq. (3.11) and the schematic representation given in Fig. 3.3, it can be concluded that the given available volume at an arbitrary slice is defined as:

$$
\tilde{V}_{c}(i)=2 \kappa \sigma_{y} d x
$$




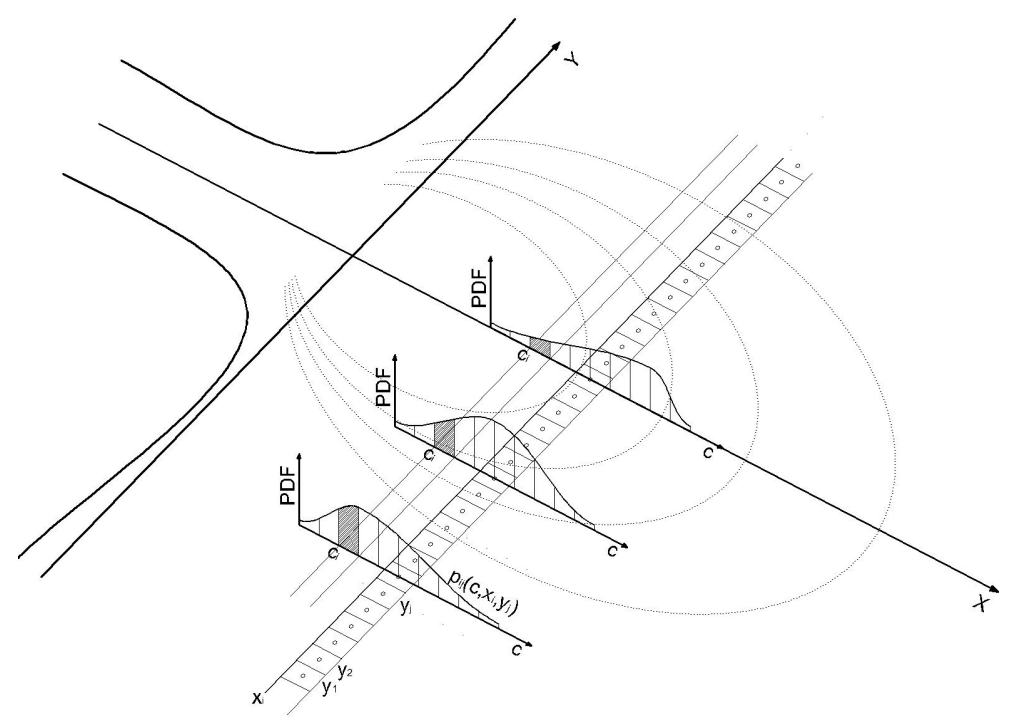

FIGURE 3.3: Schematic representation of spatially integrated pollution concentration PDFs for a chosen downstream slice.

where $\kappa$ is the number of spatial transversal concentration variance $\sigma_{y}$ taken into account.

By implementing the Eq. (3.11) continuously along the downstream slices, the corresponding EMFs are obtained. The example results are seen in Fig. 3.4, and the rest are shown in Chapter 5. While observing the EMF functions (Fig. 3.4), it can be noticed that initially there is a mass of pollutant with a higher concentration, but then it is systematically shifted to lower values of concentration in further profiles.

\subsection{Spatially integrated concentration moments $-K_{n}$}

Spatially integrated concentration moments were introduced in Eq. (3.8), where it is obvious that they are literally obtained from point PDF moments. Therefore, a straightforward way of calculating them would be to simply use the point moments derived in Chapter 2 and integrate them over all space equivalent of volume, which is shown in Section 3.3.1. However, if an analogy is to be built with point PDF moments and their recursive evaluation as described in Chapter 2, one is encouraged to try a similar approach in an integrated formulation, which is what is demonstrated in Section 3.3.2. Depending on the level of computational integration possibilities, the results for both approaches should be very similar. The final application of spatially integrated moments, regardless of the 


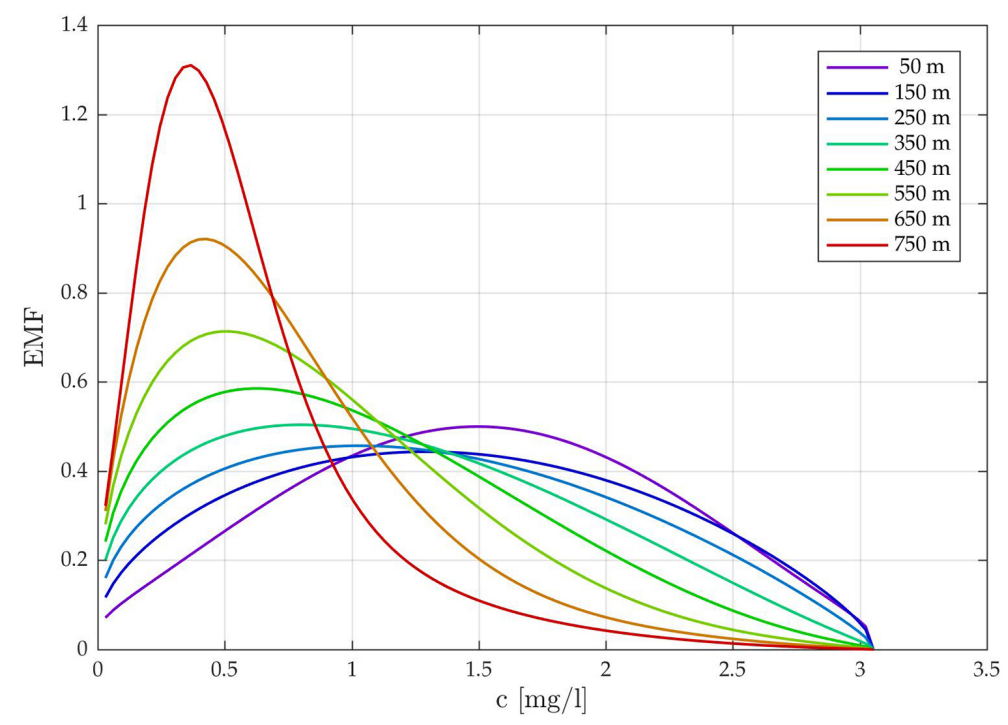

FIGURE 3.4: Example EMFs obtained by direct integration of point PDFs.

manner in which they were obtained, is certainly the EMF itself, which is presented in Section 3.3.3 in several examples, while additional results are shown in Chapter 5 .

\subsection{1 $\quad K_{n}$ obtained from point moments}

Direct integration of absolute concentration moments is applied in order to obtain the spatially integrated moments following the Eq. (3.8) for the case of contaminant plume. It is clear that the first two spatially integrated moments deliver the volume $\tilde{V}_{c}$ and the steady mass loading $L$ (see Fig. 3.5), where volume is a function of time $(t=t(x))$, while mass loading is conserved from step to step.

Hence, volume and mass loading as the functions of $x$ are written as:

$$
\begin{aligned}
& K_{0}(x)=\int_{V} \overline{c^{0}} d V=\int_{0}^{\Delta x} \int_{0}^{2 \kappa \sigma_{y}} \int_{0}^{z} \delta(z) d z d y d x=2 \kappa \sigma_{y}(x) \Delta x=\tilde{V}_{c}(x) \\
& K_{1}(x)=\int_{V} m_{1} d v=\int_{0}^{\Delta x} \int_{0}^{2 \kappa \sigma_{y}} \bar{c} d y d x=L(x)=\text { const. }
\end{aligned}
$$



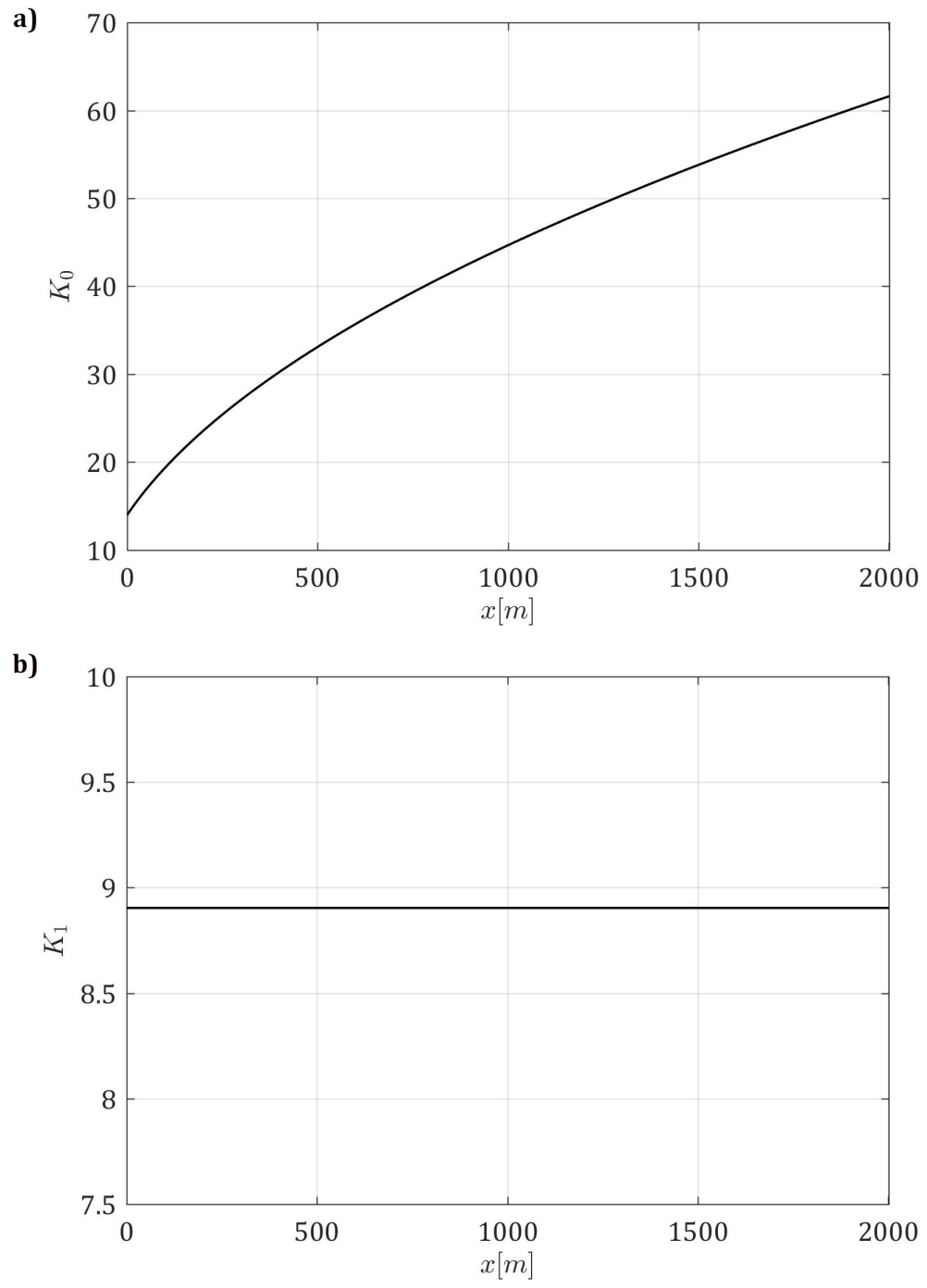

FIgURE 3.5: a) Volume $\left(K_{0}(x)\right)$; b) mass $\left(K_{1}(x)\right)$ as a function of the section.

Whereas the additional three spatially integrated moments as functions of $x$ are written as:

$$
\begin{aligned}
& K_{2}(x)=\int_{V} m_{2} d v=\int_{0}^{\Delta x} \int_{0}^{2 \kappa \sigma_{y}} m_{2} d y d x \\
& K_{3}(x)=\int_{V} m_{3} d v=\int_{0}^{\Delta x} \int_{0}^{2 \kappa \sigma_{y}} m_{3} d y d x \\
& K_{4}(x)=\int_{V} m_{4} d v=\int_{0}^{\Delta x} \int_{0}^{2 \kappa \sigma_{y}} m_{3} d y d x
\end{aligned}
$$




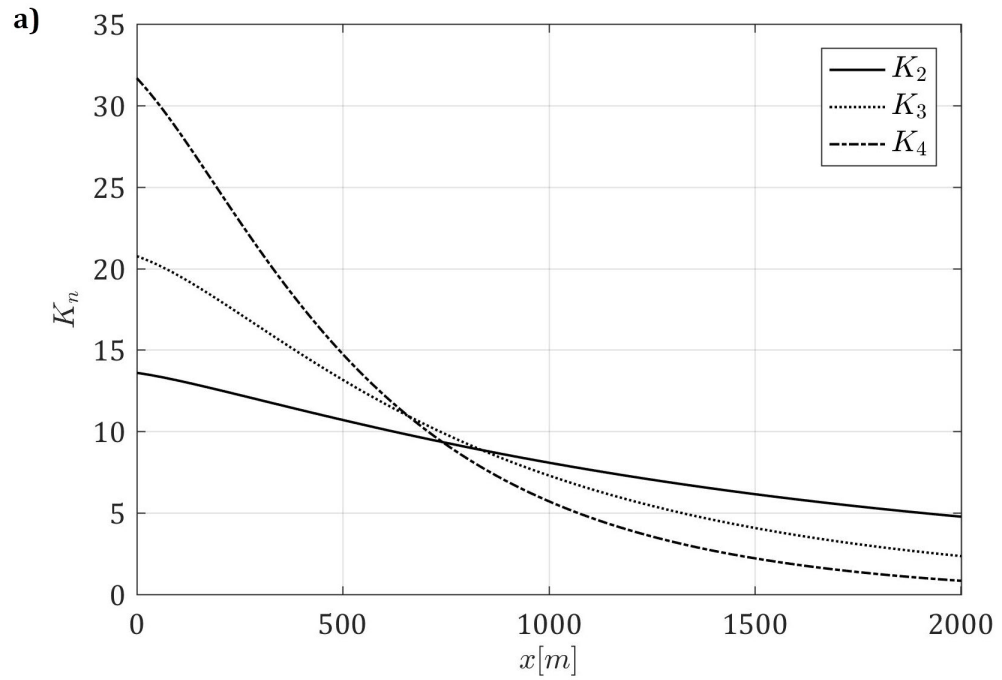

b)

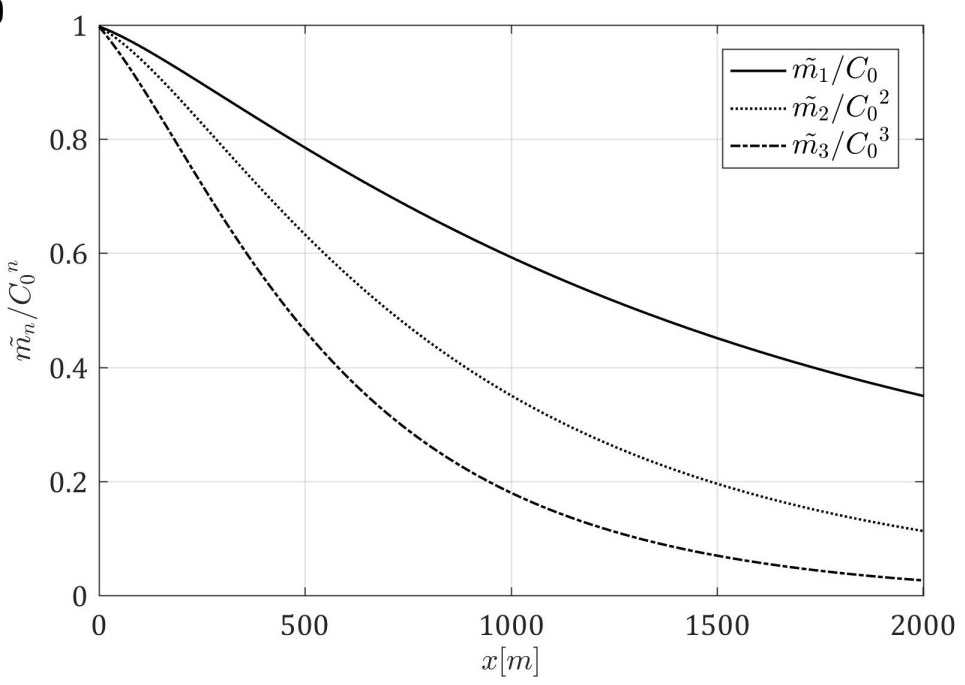

FIGURE 3.6: a) Spatially integrated moments $\left(K_{n}(x)\right)$; b) corresponding EMF moments $\left(\tilde{m}_{n}(x)\right)$ as a function of the section and obtained by integration of point moments.

The examples of such obtained moments for $K_{2}, K_{3}$, and $K_{4}$ are shown in Fig. 3.6 a. The corresponding EMF moments defined by Eq. (3.4) and normalized by $C_{0}$ are given in Fig. 3.6 b. Both representations of spatially integrated statistics exhibit the expected diminishing behavior as one moves downstream. These examples are calculated using absolute moments for the specific case of constant mean velocity as described by Eq. (2.15). 


\subsection{2 $K_{n}$ obtained using a recursive model}

Spatial integration is a convenient solution for dealing with closure problems that are present in the evaluation of point concentration statistics, particularly the point PDF. It requires fewer realizations since it eliminates the spatial reference. Following an approach similar to recursive formation of the point PDF moments, Eq. (2.5) is integrated over all space:

$$
\frac{\partial}{\partial t} \int_{V} m_{n+1}(\mathbf{x}, t) d V=-n(n+1) e_{m} \int_{V} \overline{c^{n-1}(\mathbf{x}, t)[\nabla c(\mathbf{x}, t)]^{2}} d V
$$

for $n \geq 1$, and the dependence on $(\mathbf{x}, t)$ is hereafter omitted for the sake of simplicity. Every part of the expression has previously been explained in Chapter 2 . The integral on the right side is intrinsically positive and it clearly shows that molecular diffusion, represented by the coefficient $e_{m}$, is the only means by which the concentration is reduced and the contaminant field is diluted. It can be noticed that the integrated form of Eq. (2.11), i.e. the Eq. (3.15), lacks the advective term $\nabla \cdot \overline{v c^{n+1}}$ which is diminished by integration over all space. When the concentration gradient from Eq. (2.9) is introduced to Eq. (3.15), it becomes:

$$
\frac{\partial}{\partial t} \int_{V} m_{n+1} d V=-n(n+1) \frac{e_{m}}{\lambda^{2}} \int_{V} \overline{c^{n+1}-2 c^{n} c_{t}+c^{n-1} c_{t}^{2}} d V
$$

Using previously defined $K_{n+1}(t)=\int_{V} m_{n+1}(\mathbf{x}, t) d v$ by Eq. (3.8) and introducing the dimensionless timescale $\tau=\frac{e_{m}}{\lambda^{2}} t$, Eq. (3.16) becomes a first-order differential equation for spatially integrated concentration moments:

$$
\frac{\partial K_{n+1}(\tau)}{\partial \tau}+k K_{n+1}(\tau)=k\left[2 K_{n}(\tau) c_{t}-K_{n-1}(\tau) c_{t}^{2}\right]
$$

where $k=n(n+1)$. Such an equation provides a recursive solution for spatially integrated moments:

$$
K_{n+1}(\tau)=e^{-k \tau}\left(k \int_{0}^{\tau}\left[2 K_{n}(\zeta) c_{t}-K_{n-1}(\zeta) c_{t}^{2}\right] e^{k \zeta} d \zeta+\mathrm{K}_{n+1}(0)\right)
$$

and for $n \geq 1$. The recursive solution defined by Eq. (3.18) can be used in any case of the contaminant dilution process for obtaining spatially integrated moments if one is aware of the initial solutions, i.e. $K_{n+1}(0)$. 
For the case of contaminant plume in a river-dominated estuary, where time is expressed as a function of $x$, with a continuous steady line source, two cases are explored, following the characterization of the mean flow velocity described in Section 2.1.

\section{Concentration moments for the specific case of constant mean velocity}

The specific case of constant mean flow velocity $U$ dependent on the distance ( $x$ ) from the river mouth or source, causes the Eq. (3.17) to be written:

$$
\frac{\partial K_{n+1}(x)}{\partial x}+\frac{k \alpha}{U} K_{n+1}(x)=\frac{k \alpha}{U}\left[2 K_{n}(x) c_{t}-K_{n-1}(x) c_{t}^{2}\right]
$$

which then has a recursive solution in the following form:

$$
\begin{aligned}
K_{n+1}(x) & =e^{-\frac{k \alpha}{U} x} \\
& \times\left(\frac{k \alpha}{U} \int_{0}^{x}\left[2 K_{n}(\xi) c_{t}-K_{n-1}(\xi) c_{t}^{2}\right] e^{\frac{k \alpha}{U} \xi} d \xi+K_{n+1}(0)\right)
\end{aligned}
$$

\section{Concentration moments for a non-uniform mean velocity}

This case shows a more general behavior with a non-uniform mean velocity in the $x$ direction $U(x)=U_{0} e^{-\nu x}$ (Eq. (2.17)), where $U_{0}$ is the cross-sectionally averaged velocity at the river mouth and $\nu\left[\mathrm{m}^{-1}\right]$ is an attenuation coefficient representing different mechanisms that, combined, progressively reduce the mean velocity in the seaward direction [58]. Using $t=t(x)$, as described in Appendix B.2, the equation Eq. (3.17) becomes:

$$
\frac{\partial K_{n+1}(x)}{\partial x}+\frac{k \alpha}{U_{0} e^{-\nu x}} K_{n+1}(x)=\frac{k \alpha}{U_{0} e^{-\nu x}}\left[2 K_{n}(x) c_{t}-K_{n-1}(x) c_{t}^{2}\right]
$$

which, again, has a recursive solution in the form:

$$
\begin{aligned}
K_{n+1}(x) & =e^{-\frac{k \alpha}{U_{0} \nu}\left(e^{\nu x}-1\right)} \\
& \times\left(\frac{k \alpha}{U_{0}} \int_{0}^{x}\left[2 K_{n}(\xi) c_{t}-K_{n-1}(\xi) c_{t}^{2}\right] e^{\left[\frac{k \alpha}{U_{0} \nu}\left(e^{\nu \xi}-1\right)+\nu \xi\right]} d \xi+K_{n+1}(0)\right)
\end{aligned}
$$




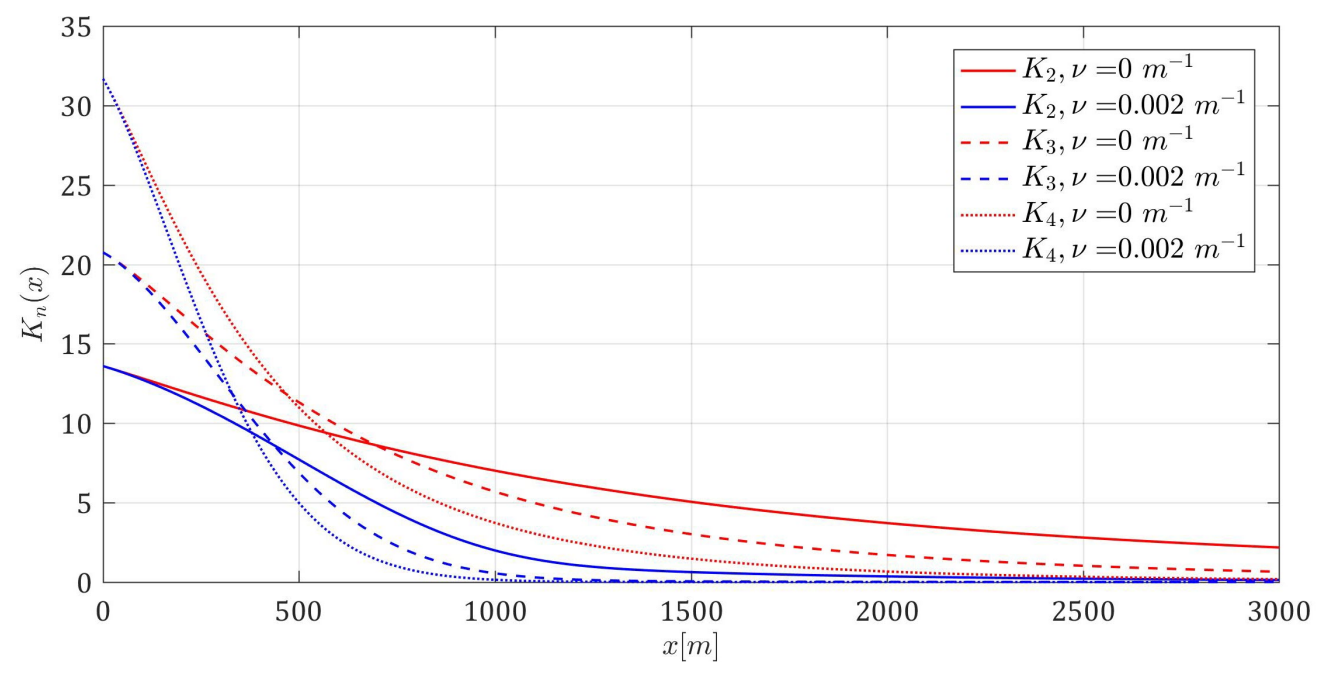

FIGURE 3.7: Spatially integrated moments $K_{n}(x)$, obtained by a recursive solution for constant mean velocity (red) and non-uniform mean velocity (blue).

Several examples of spatially integrated moments for the first case are provided in Appendix C.1 and for the second case in Appendix C.2. For both cases, $\alpha=e_{m} / \lambda^{2}$ and initial values for cross-sectionally integrated concentration moments for $n \geq 1$ are defined as (see Appendix C.3 for details):

$$
K_{n+1}(0)=\frac{L^{n+1}}{\left(\sigma_{y_{0}} \sqrt{2 \pi}\right)^{n}} \operatorname{erf}\left(\frac{\kappa}{\sqrt{2}}\right)
$$

In both Eq. (3.20) and Eq. (3.22) the integrated concentration moments $K_{n}(x)=$ $\int_{V} m_{n}(\mathbf{x}, t(x)) d V$ are defined cross-sectionally and $d V=d x d y d z$ is the available volume as a depth-integrated surface slice which is $\Delta x$ wide in the stream flow direction $(x)$ and $2 \kappa \sigma_{y}$ wide in a transversal direction $(y)$. The comparison between those moments can be seen in Fig. 3.7, which shows that velocity attenuation causes moments to diminish more quickly at smaller distances from the source, contrary to the constant velocity case.

What is important to notice is that both ways of obtaining spatially integrated concentration moments $K_{n}(x)$, direct integration of point moments (Section 3.3.1), or the recursive solution in Section 3.3.2 deliver similar results. However, direct integration is time-consuming and requires more computational resources, while the recursive solution delivers results within seconds. Hence, the recursive solution is used hereafter as a means of obtaining spatially integrated moments, from which the EMF is then obtained using the moment inversion procedure. 


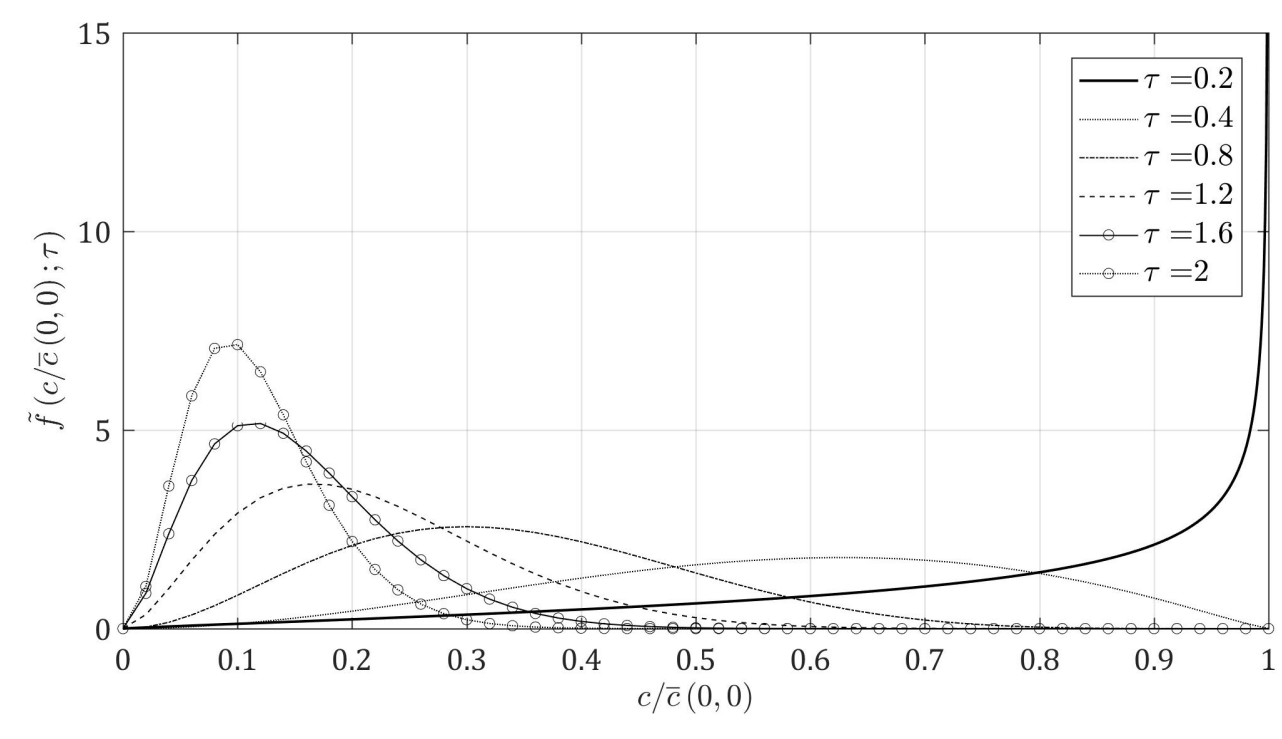

FIGURE 3.8: EMF examples for contaminant plume release as a function of dimensionless time $\tau$.

\subsubsection{Moment inversion to obtain the EMF}

Since it has been shown how the EMF is similar to the PDF (Eq. (3.7), it is expected to use the same methodology for obtaining one by inversion of its lower moments. In Section 2.3.1, Beta distribution is represented as a convenient and rewarding way of obtaining the PDF from its lower moments [105][13][133],[124][140].

In Fig. 3.8, for the more general case where $\tilde{f}$ depends on dimensionless time $\tau$, it shows a shift from the initial time (near source cross-sections) where most of the mass is around the source maximum concentration toward lower concentration values as a result of a stronger dilution process when $\tau$ increases. For instance, the cross-section corresponding to $\tau=2.0$ has most of the mass with the concentration values around one tenth of the initial value.

In order to represent the effect of velocity attenuation along with the potential application of the EMF as a dilution measure, Fig. 3.9 is given for a hypothetical nutrient plume release in the Žrnovnica estuary. A detailed description of that case study is produced in Chapter 4, while additional results can be found in Chapter 5. However, the example in Fig. 3.9 a. exhibits an interesting effect of the attenuated velocity field defined by the coefficient $\nu=0.002 \mathrm{~m}^{-1}$. Again, it contracts the plume closer to the source, but due to its definition given by Eq. (2.17), it has a very small to non-existent effect close to the river mouth. Hence, the EMFs for $x=50 \mathrm{~m}$ are almost the same for both the constant and the non-uniform mean velocity. However, moving in the seaward direction, other physical processes (e.g. wind-induced currents) reduce the velocity, which contributes to the 

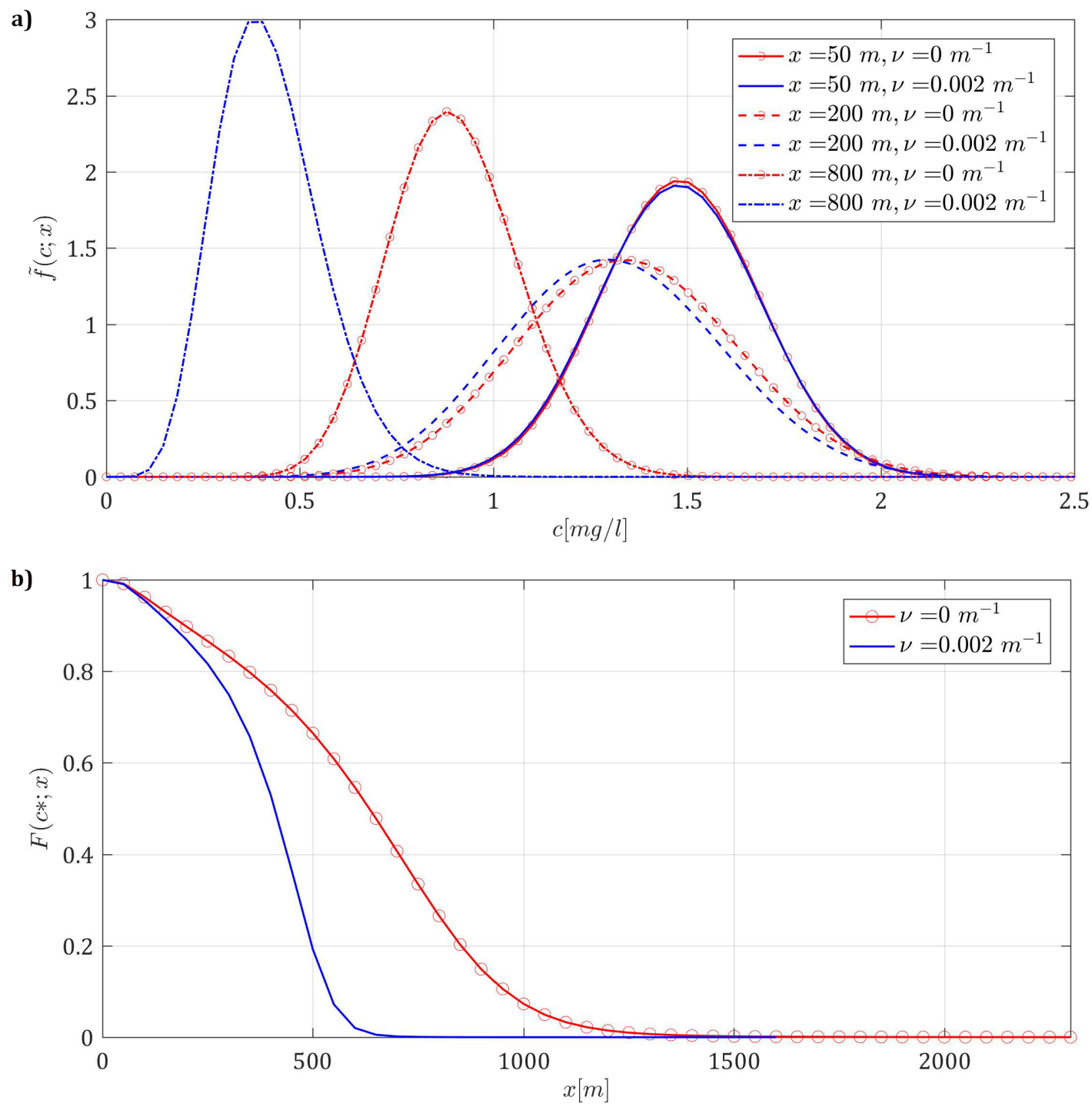

FIGURE 3.9: a) EMF examples as a function of distance from the source $x ; \mathrm{b})$ The fraction of the release mass above the chosen concentration limit of $1 \mathrm{mg} / 1$, for constant mean velocity (red), and nonuniform mean velocity (blue).

dilution process by further lowering concentration values at farther cross-sections in contrast to the uniform mean velocity solution.

The mass fraction containing the concentration above an arbitrary value (in this case for the total nitrogen loading $c^{*}=1 \mathrm{mg} / 1$ [89]), as defined by Eq. (3.6), now implies the potential measure of dilution which can be seen in Fig. $3.9 \mathrm{~b}$. The fact that the dilution process is increasingly moving away from the river mouth was not surprising, but the way in which different mechanisms may affect the dilution process is very important to understand. Molecular diffusion 
is a slow-acting process and mean velocity attenuation, as already mentioned, requires some distance from the river mouth for its action to begin. Due to the fact that hydrodynamics of the open waters take over and further increase the dilution mechanism at further cross-sections, the mass fraction above the considered limit is rapidly reduced in the non-uniform mean velocity case. Finally, it can be expected that no mass with concentration above $c^{*}$ will be found at distances beyond $1500 \mathrm{~m}$ for constant mean velocity, or at distances beyond $800 \mathrm{~m}$ for non-uniform mean velocity. This type of information might have a major impact when considering different extensions of anthropological activities in the coastal area surrounding an estuary of interest. 


\section{Chapter 4}

\section{Field measurements and numerical modeling}

In order to test and validate methodologies for obtaining the concentration point and spatially integrated statistics which were developed in Chapter 2 and Chapter 3, several different procedures are conducted in this chapter. Several measurement campaigns (Section 4.2) and two different numerical models are implemented (Section 4.3) at the chosen case study location. Since the measurements and modeling that were used in the verification process are applied to the Žrnovnica River estuary, the first section provides their detailed description. Furthermore, Section 4.1 presents sources for additional data in this area that was required for the modeling and measuring procedures. A set of illustrative examples for these procedures in comparison to developed methodologies will be presented in Chapter 5.

\subsection{Case study - Žrnovnica River estuary}

The Žrnovnica River is a relatively small river which flows into Stobreč Bay (see Fig. 4.1), near the city of Split, the second largest city in Croatia. The geological background of this area is mostly karstic, occasionally with flysch layers. A large uncontrolled landfill is located less than $2 \mathrm{~km}$ away from the Žrnovnica River. The local municipality has implemented a wastewater power plant which only works on primary treatment. Possible contaminant influx from different sources and riverine pollutants represent a severe hazard for the Žrnovnica estuary, especially given a rather small river outflow (annual average $2 \mathrm{~m}^{3} / \mathrm{s}$ ) and a limited depth of the bay. This area already has records of pollution [99] as a result of anthropogenic activity creating a vulnerable ecosystem. Nevertheless, the nearby communities of Stobreč, Žrnovnica, and Podstrana have a highlydeveloped tourist industry, which ensures that even more pressure will be put on the estuary and the bay. Even the initial water quality at the Žrnovnica spring 


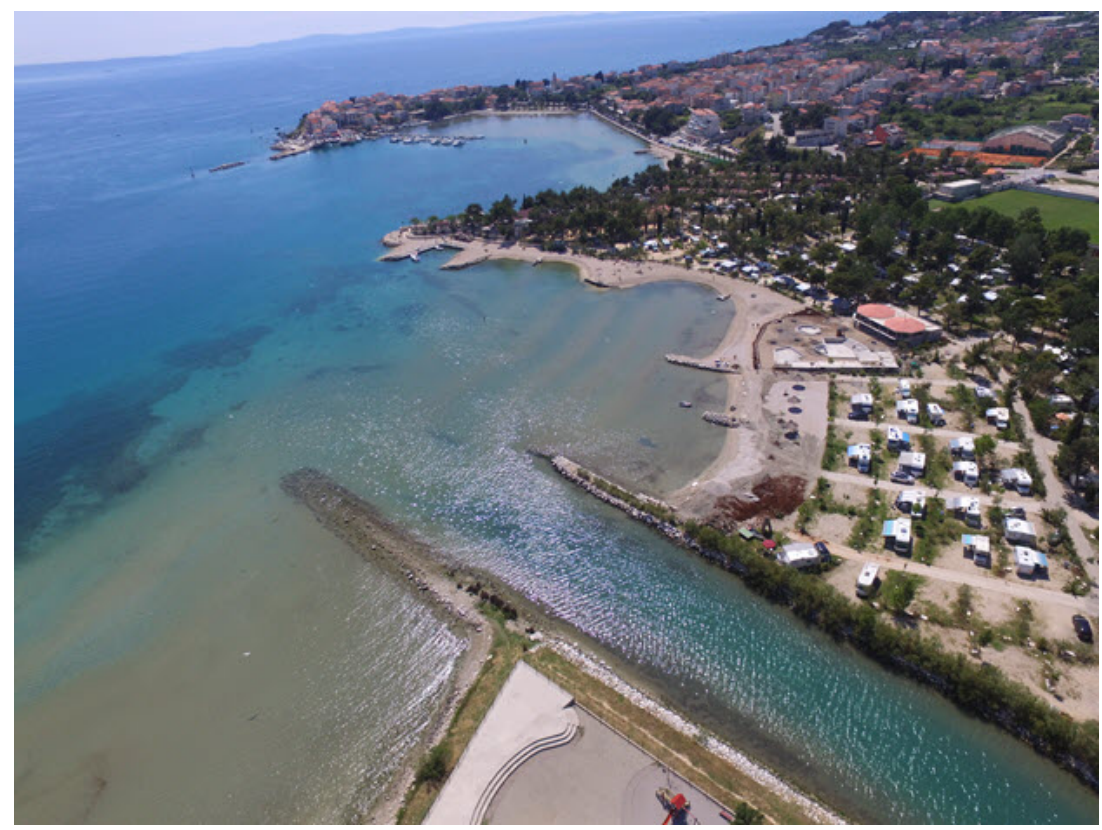

FIGURE 4.1: Aerial view of the Žrnovnica River estuary (courtesy of Flickr).

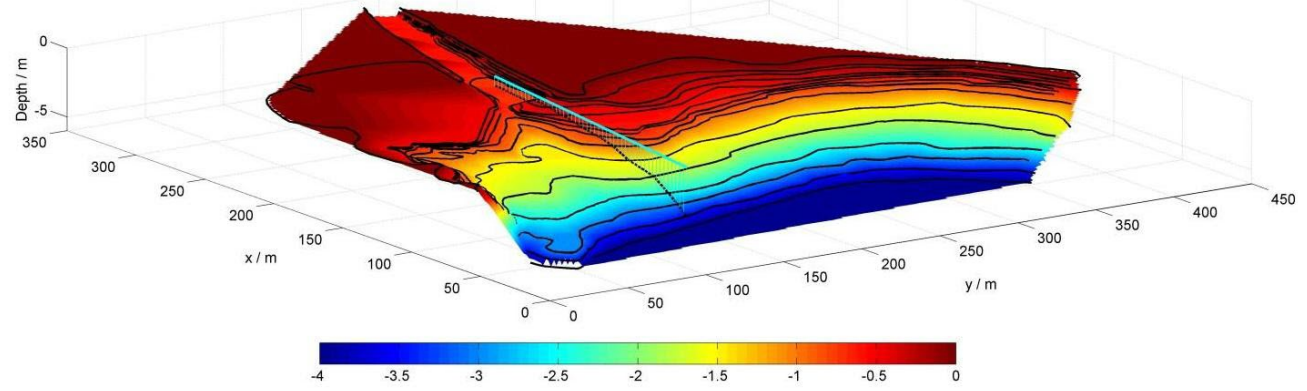

FIGURE 4.2: 3D depth distribution in the investigated area.

is compromised by inadequate sewage systems in the region [91].

Several key pieces of information were necessary for measurements and modeling:

- bathymetry

- wind speed

- high and low water

- weather forecast 


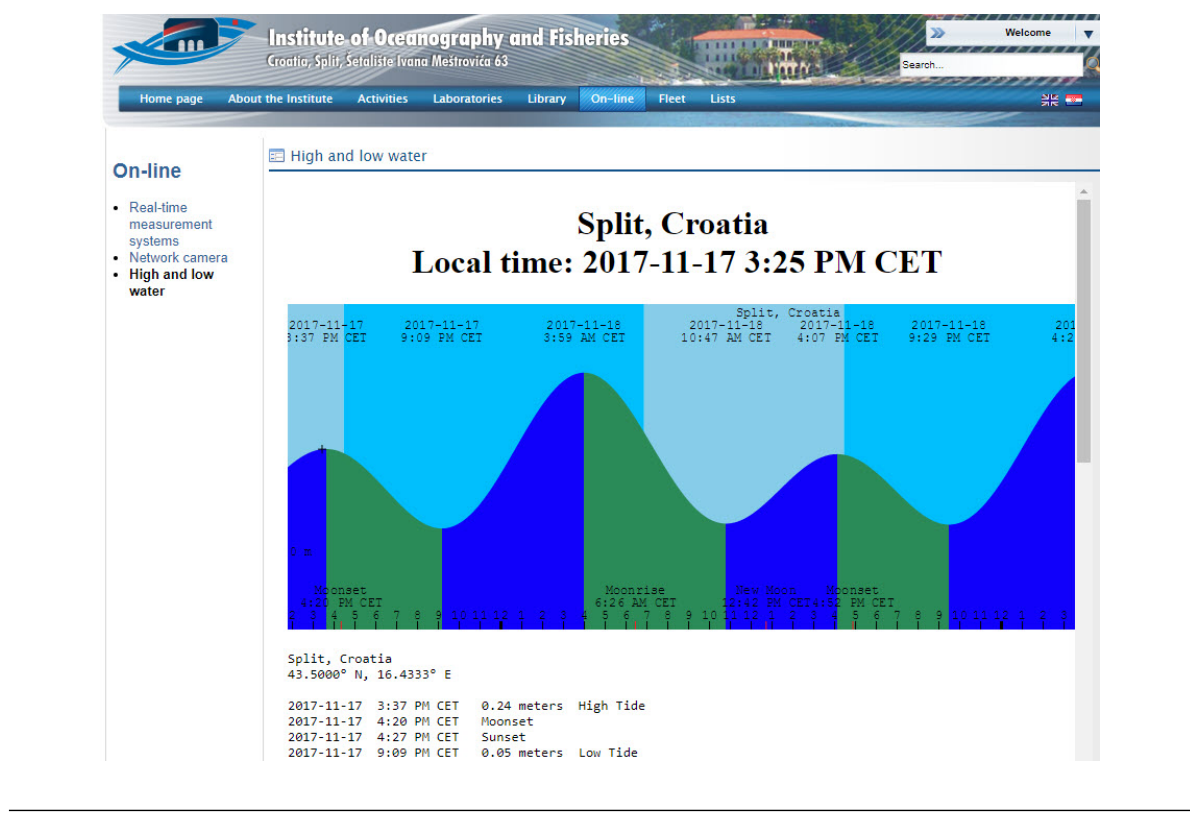

FIGURE 4.3: Example of online data for high and low water (courtesy of IOF).

- river flow

Following that list, the sources for each item of information are presented below.

The bathymetry data (see Fig. 4.2) was obtained from the coastal design project for the municipality of Podstrana, which was previously done by the Faculty of Civil Engineering, Architecture and Geodesy in Split ([49]).

For wind speed and water levels data (example given in Fig. 4.3), the services of a local institute, namely the Institute of Oceanography and Fisheries (IOF) were used [111], since their measuring station is very close to the case study site (approximately $10 \mathrm{~km}$ ). Weather forecast was needed when measurement campaigns were planned, which is why online data from an official Croatian agency (Meteorological and Hydrological Service, DHMZ) was used [103]. The hydrological branch of that agency provided the river flow data for the Žrnovnica [102]. The flow dataset for the year 2015 is shown in a screenshot of the official site (see Fig. 4.4), along with other attribute data for the measuring station.

This section presented all the relevant data with regard to the case study of the Žrnovnica estuary. The following two sections refer to and explain in more detail, if needed, any additional data specifically needed for measurement or modeling. 


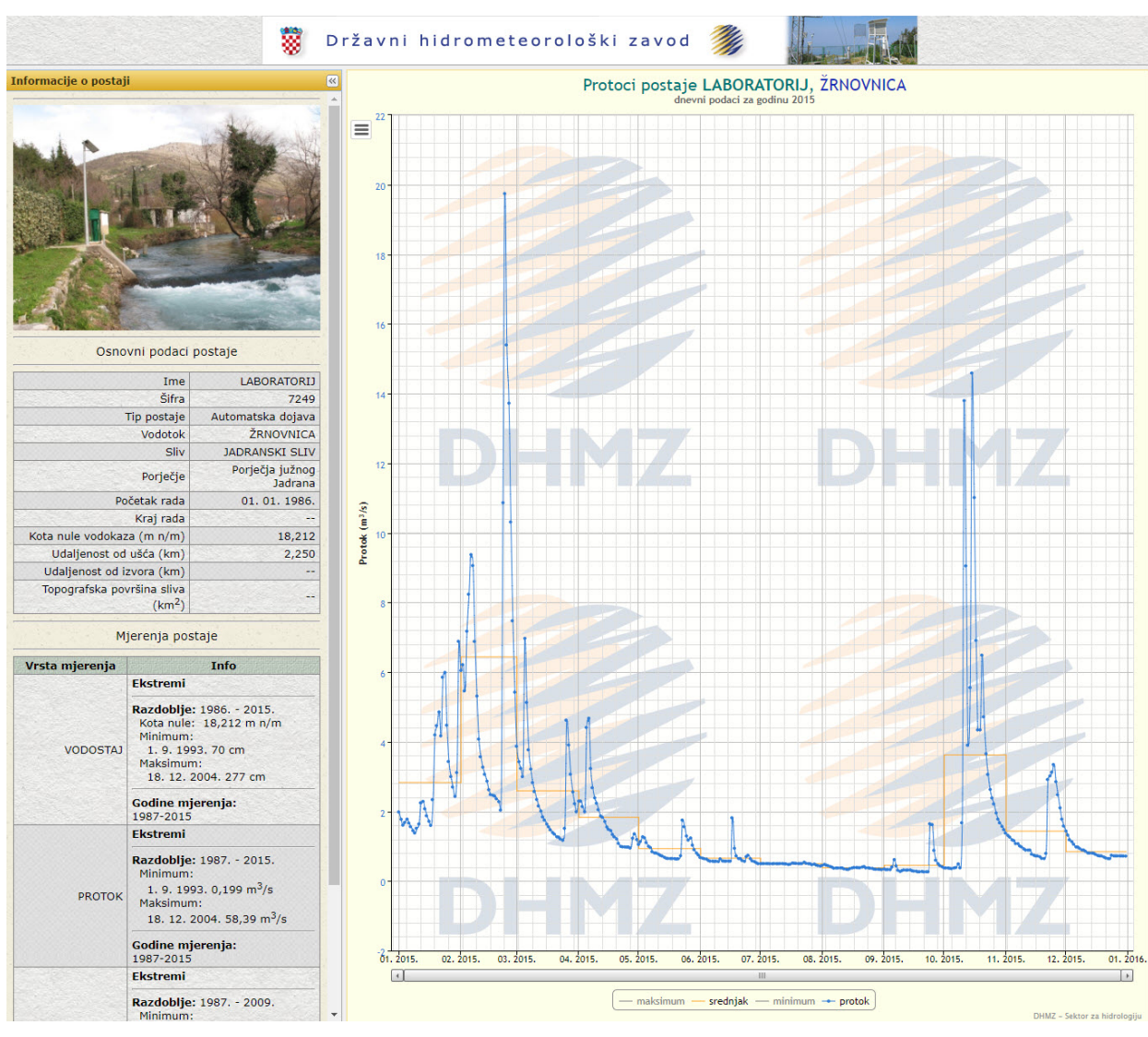

FIGURE 4.4: Žrnovnica flow dataset for the year 2015 (courtesy of the Croatian Meteorological and Hydrological Service).

\subsection{Field measurements}

While investigating the options for validating the proposed methodologies, different approaches came to light. Tracer tests were significantly complicated and expensive due to the requirement of a large number of realizations. Moreover, the area's sensitivity (tourism, social interests, etc.) asked for a less intrusive approach. Thus, the initial idea was to only measure water velocities (Section 4.2.1) in order to gain insight into the hydrodynamic properties of the system, and then to apply them to a random walk particle tracking model (Section 4.3.1). Afterwards, within the CPoRT project (Chapter 6) realization, a new idea emerged. The advection and diffusion processes of a potential contaminant entering the sea via the river are similar to those of sea salinity reduction caused by freshwater input; therefore, a proxy concentration approach using salinity data is implemented in Section 4.2.2. 

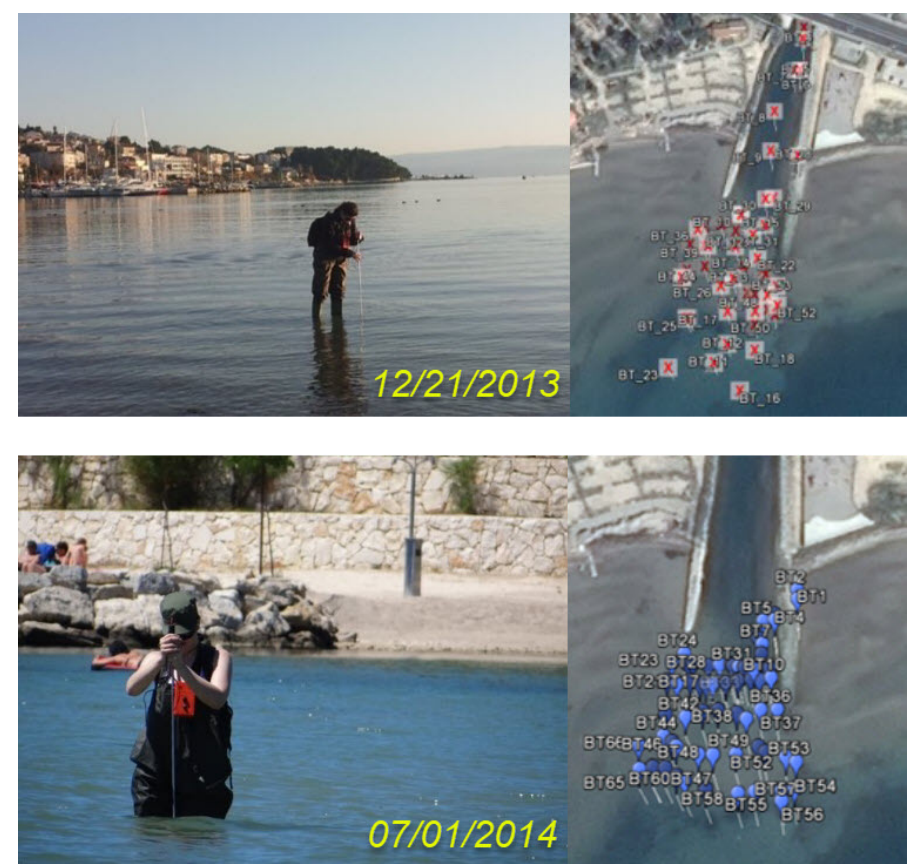

FIGURE 4.5: Velocity measurements procedure and the recorded positions for the winter (up) and summer (down) measuring campaigns.

\subsubsection{Setup for velocity measurements}

Velocity measurements were used as a preliminary validation tool, whereby the concept of mean velocity attenuation presented in Chapter 2 was observed and mathematically defined afterwards.

Two series of velocity measurements were performed in the winter and in the summer, respectively. The first set of measurements was taken on December 21,2013 , for low tide conditions (0.05 m of amplitude), wind speed of $1.4 \mathrm{~m} / \mathrm{s}$, and a river discharge flow rate of $1.6 \mathrm{~m}^{3} / \mathrm{s}$. The second set of measurements was taken on July 1, 2014, at low tide ( $0.15 \mathrm{~m}$ of amplitude), wind speed of 2.2 $\mathrm{m} / \mathrm{s}$, and a river flow rate of $2.4 \mathrm{~m}^{3} / \mathrm{s}$ due to intense rainfall. Measuring was in both cases performed mostly on foot, with the exception of the first several ones in the winter, which were conducted on a small boat (courtesy of Hrvoje Stipančević). The positions of recorded data along with the example photos are shown in Fig. 4.5.

At each measurement point, velocity was measured using a hydrometric wing 
TABLE 4.1: The properties of velocity measurement campaigns.

\begin{tabular}{lll}
\hline Date & Number of points & River flow \\
\hline $12 / 21 / 2013$ & 43 & $1.6 \mathrm{~m}^{3} / \mathrm{s}$ \\
$07 / 01 / 2014$ & 59 & $2.4 \mathrm{~m}^{3} / \mathrm{s}$ \\
\hline
\end{tabular}
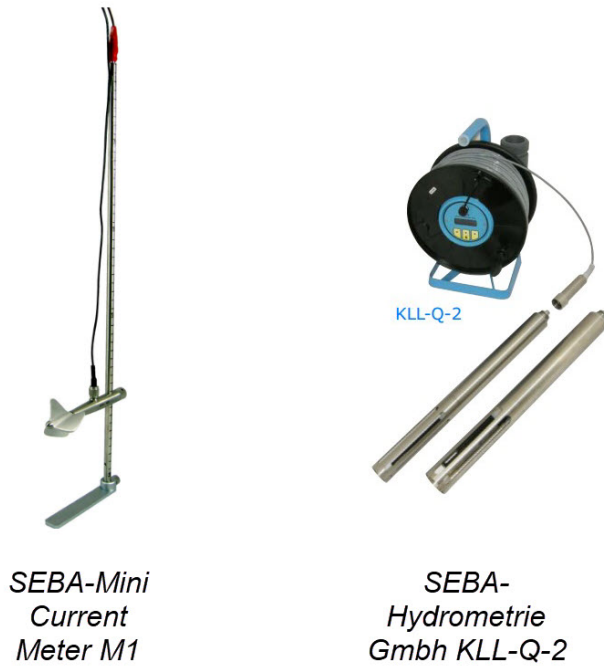

FIGURE 4.6: Hydrometric wing (left) and salinity probe (right).

(SEBA-Mini Current Meter M1 with an initial velocity of $0.025 \mathrm{~m} / \mathrm{s}$ ) with an averaging interval of $60 \mathrm{~s}$, and georeferenced by GPS (PerformanceBox with an accuracy of $5 \mathrm{~m}$ ). The obtained data showed the dominant role of stream flow velocity, as the velocities measured perpendicular to the plume axis $x$ were negligible. Most of the flow was carried in an upper shallow layer of freshwater $(10-30 \mathrm{~cm})$. Velocities for points deeper than $30 \mathrm{~cm}$ with respect to the water surface were undetectable, and therefore neglected. The properties of these two velocity measurement campaigns are presented in Table 4.1. There were more GPS records (see Fig. 4.5), but only effective points with accurately obtained data were taken into account.

In order to detect a stratified flow, salinity was measured using a SEBA Hydrometrie Gmbh KLL-Q-2 probe in ten randomly chosen points in front of the river mouth, at three different depths. Both instruments used for the velocity and salinity data are shown in Fig. 4.6.

The hydrometric wing was used due to low velocities and because brackish water had shown disruptive effects on the ADV (Acoustic Doppler Velocimeter, SonTek), while ADCP (Acoustic Doppler Current Profiler) was unusable due to 
shallows causing too big a shadow in the measuring profile. In general, velocity measurements have shown to be consistent with the proposed models used in Chapter 2 and Chapter 3. Nevertheless, the results and the implementation of measured data in models are presented in Chapter 5, along with different comparisons and a discussion.

\subsubsection{Setup for salinity measurements}

Within the preliminary verification process set out in the previous section, some initial salinity measurements were used as support data to justify the stratified conditions in the estuary. However, sea water dilution (supported by salinity data) may be considered as an inverse process to river transport by freshwater discharge. There are some studies using salinity as a measure for detecting different properties of hydrodynamic behavior in rivers and estuaries. Hence, salinity measurements were used for an estimate of the dispersion coefficient by $[148,78]$. Cross-sectionally averaged salinity over a representative time period was used as a proxy for a continuous record of mean salinity by [62]. In a study carried out by [152], salinity was used as a critical factor in understanding and predicting physical and biogeochemical processes in the Chesapeake Bay, USA. More recently, the change in surface sea salinity (SSS) was used as an indicator of extreme freshwater outflow when modeling the potential flood hazard due to typhoons in the Japan coastal area [146]. In the research covered by this thesis and the CPoRT project, salinity is introduced as a proxy for a normalized concentration in the surface layer as follows:

$$
c_{p}(\mathbf{x}, t)=1-\frac{S(\mathbf{x}, t)-S_{\min }}{S_{\max }-S_{\min }}
$$

where $S(\mathbf{x}, t)$ is the measured salinity at sampling points, $S_{\min }$ is the salinity measured at the river mouth, and $S_{\max }$ represents the sea salinity in the estuary. Such utilization of salinity data is possible only when the steady-state assumption is valid, such as when wind currents and tides have a minimal effect on the estuary [148]. To ensure a steady flow and water levels of the river, an adequate time period from the most recent precipitation was required. Therefore, weather forecast and tide predictions (Fig. 4.3) were taken into account when choosing dates for the measurement campaigns.

Since steady state with most of the transport happening in the upper layer of the stratified flow is assumed, there was a need for a system of keeping the salinity probe at the same depth for all measurements. The Seahawk SBE37 probe 


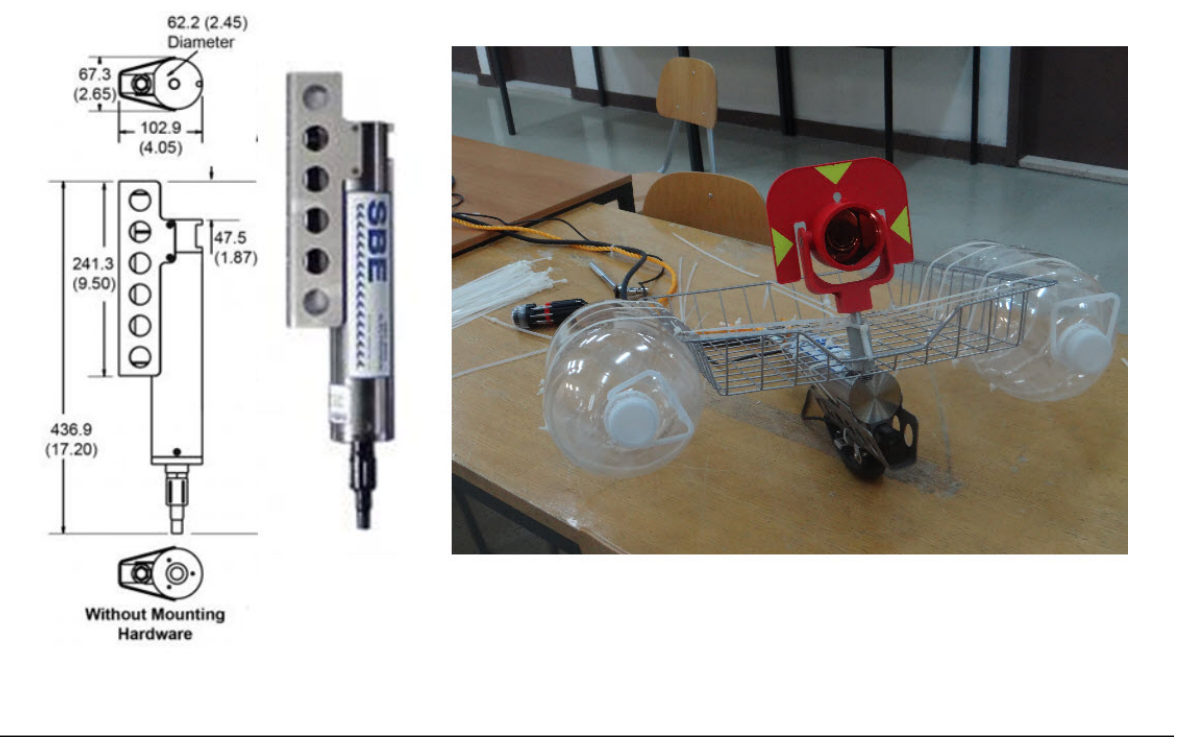

FIGURE 4.7: Salinity probe (left) and the measurement setup in the laboratory (right).

was used in a special floating cage in order to ensure the same depth continuously (see Fig. 4.7). These measurement campaigns were part of the CPoRT project, which enabled the procurement of some of the necessary equipment and services, including an inflatable boat with an outboard engine, a combined GPS and sonar device (Garmin EchoMap42dv), a portable computer and geodetic surveying as support. A prism positioned on the top of the floating cage (Fig. 4.7) was used to pinpoint the location from land by surveyors, and the ultrasonic depth probe was positioned just below it. Geodetic surveying was used in two campaigns in order to determine the level of accuracy of the GPS device. It showed a circular deviation of $3.71 \mathrm{~m}$, which is considered adequate for this kind of measurement, especially due to a small sampling period of $6 \mathrm{~s}$ which allows for the estimation of the representative volume within which the probe was operating. In general, before the field trip, a plan for covering the area was designed and the probe was set to a certain recording frequency. Furthermore, the system as a whole was tested in the lab (e.g. Fig. 4.8). The weather and river flow conditions were monitored using online data specified in the previous section. Each time, the objective was to use the 5-8 hours period of a relatively steady state with minimal tide effects.

Once in the field, the floating cage was kept slightly detached from the boat itself as can be seen in Fig. 4.9. The probe was powered by a $12 \mathrm{~V}$ battery in the boat, and also connected to an ultraportable PC. It had to be initiated by the PC, but afterwards it was autonomous thanks to the internal memory. Occasionally, data gathering would be interrupted due to the misplacement of cords or the probe 


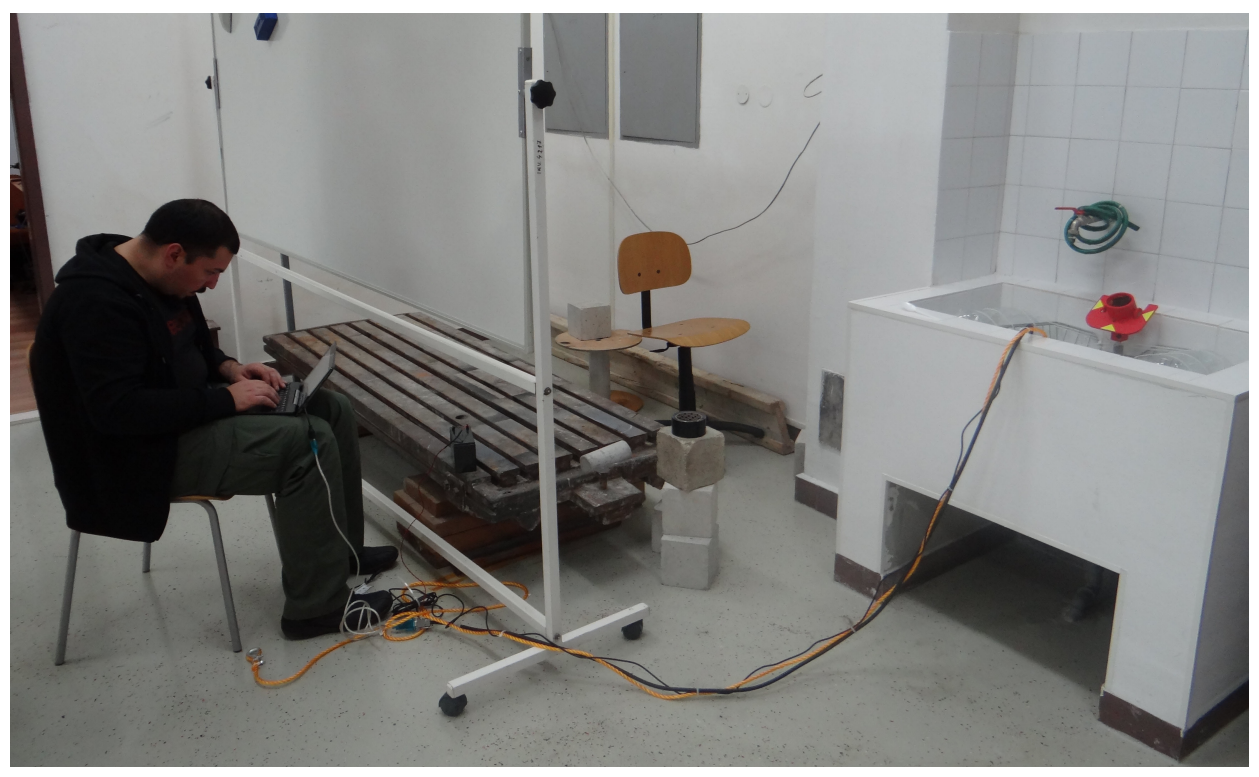

FIGURE 4.8: Salinity probe test in the laboratory.

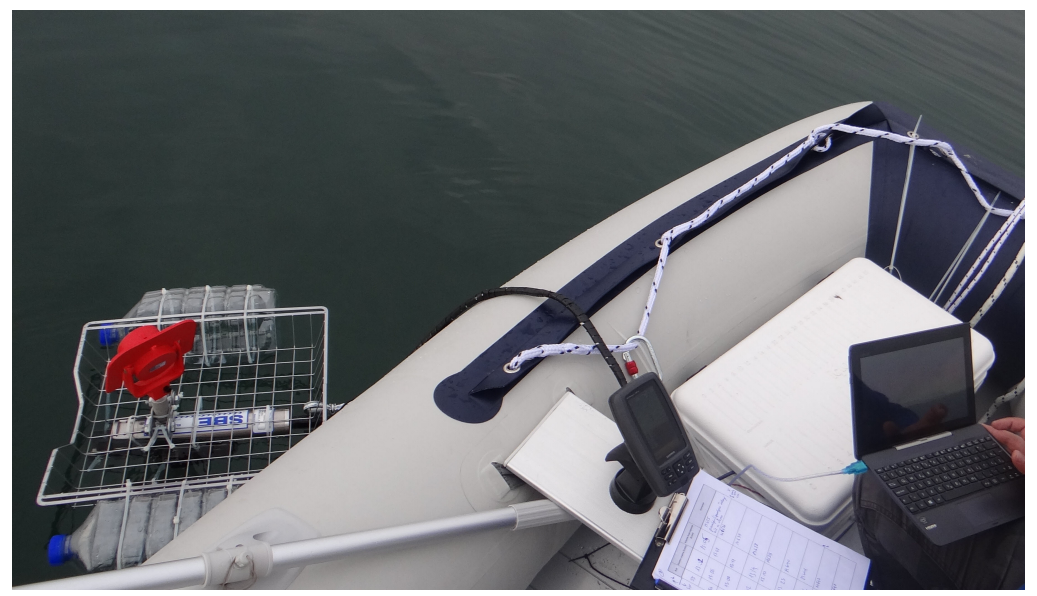

FIGURE 4.9: Salinity measurement setup in the field.

not being fully submerged. Therefore, data records were frequently monitored on the PC display to control the input. The probe was continuously collecting data on the temperature, conductivity, and depth, even when the boat was moving; however, only the selected points when the surface was calmed down after anchoring and settling the boat at the position were taken into account. Those points were marked by manual pinpointing on the GPS device at the beginning and at the end of the measuring interval. Those two GPS mark IDs per point and the corresponding time were specified in the data sheet manually as minimal data redundancy.

Since measurement options and conditions were investigated in order to meet optimum outcomes, four different measurement campaigns were conducted within 
the CPoRT project (Table 4.2):

- $1^{\text {st }}$ campaign: salinity data was gathered for two minutes per point with a 10-second sampling period; records were obtained effectively for 55 points. Since this was the first measuring process, insufficient points were successfully recorded, due to getting used to all the technical settings. The river flow was $1.08 \mathrm{~m}^{3} / \mathrm{s}$, wind speed for the 6-hour morning period (08:00 am to $02: 00 \mathrm{pm}$ ) of measurements was between 0 and $0.8 \mathrm{~m} / \mathrm{s}$ (mostly from the south-east) and it was low tide with an amplitude of less than $0.2 \mathrm{~m}$.

- $2^{\text {nd }}$ campaign: since more spatially distributed data points were needed, the sampling period was set to the probe's factory limit of $6 \mathrm{~s}$. Remaining at a recording interval of two minutes, and due to the steady state of eight hours (08:00 am to 04:00 pm) with mostly low tide conditions (amplitude of 0.07 $\mathrm{m})$, the number of recorded points increased to 105. More area was covered as can be seen in Fig. 4.10. The river flow was $0.9 \mathrm{~m}^{3} / \mathrm{s}$ and wind speed was on average $1 \mathrm{~m} / \mathrm{s}$ (mainly from the west) for that period. Just after 04:00 $\mathrm{pm}$, the wind drastically increased to $6 \mathrm{~m} / \mathrm{s}$, but all the measurements had been taken before that.

- $3^{\text {rd }}$ campaign: both of the two first campaigns focused on covering the area with as much points as possible. Since those measurements enabled only 12 data items per point in the first case, and 20 data items per point in the second case, the third campaign was conducted with up to 30 minutes per point. Naturally, that enabled a bigger set of data (about 300 records per point), but it also enabled the definition of the time interval needed for the data to converge, which is specifically shown in Chapter 5. However, this time interval drastically decreased the number of collected points to only nine; hence, they were chosen in centerline positions so that velocity can be measured there too (the same as in Section 4.2.1). These additional velocity measurements were used to verify the attenuation model from Chapter 2, with the data now obtained from positions at a bigger distance from the source, and also for comparison with the results from the MOHID model. Measurements were taken from 10:00 am to 03:00 pm in low tide conditions (amplitude of $0.04 \mathrm{~m}$ ), wind speed ranging from 0.4 to $0.9 \mathrm{~m} / \mathrm{s}$ (mostly from the north and from the north-east). The river flow was $0.72 \mathrm{~m}^{3} / \mathrm{s}$.

- $4^{\text {th }}$ campaign: the third campaign discovered that most of the data converges within 10 minutes of the recording interval (Fig. 5.29); therefore, this 
TABLE 4.2: The properties of salinity measurement campaigns.

\begin{tabular}{llll}
\hline Date & Number of points & Recording interval & River flow \\
\hline $11 / 06 / 2015$ & 55 & $2 \mathrm{~min}$ & $1.08 \mathrm{~m}^{3} / \mathrm{s}$ \\
$11 / 14 / 2015$ & 105 & $2 \mathrm{~min}$ & $0.88 \mathrm{~m}^{3} / \mathrm{s}$ \\
$12 / 29 / 2015$ & 9 & $30 \mathrm{~min}$ & $0.72 \mathrm{~m}^{3} / \mathrm{s}$ \\
$12 / 30 / 2015$ & 13 & $10 \mathrm{~min}$ & $0.72 \mathrm{~m}^{3} / \mathrm{s}$ \\
\hline
\end{tabular}

one focused on obtaining cross-sectional data (perpendicular to the centerline) if possible, while keeping the interval at 10 minutes. This time, 14 effective points were collected among which six per two cross-sectional profiles. Each point had around 100 records which were afterwards used to obtain point and spatially integrated statistics of proxy concentration for comparison with analytical models developed in Chapter 2 and Chapter 3. Since it took place just a day after the third campaign, the river flow was still 0.72 $\mathrm{m}^{3} / \mathrm{s}$, the wind mostly from the east (it had the characteristics of the local Bora wind) was stronger and demonstrated fluctuating velocities between 2 and $4 \mathrm{~m} / \mathrm{s}$. During the measurement period from 10:00 am to 02:00 pm, the tide was low with an amplitude of $0.06 \mathrm{~m}$.

All campaigns with basic data are listed in Table 4.2 while the area covered by each field trip is presented in Fig. 4.10.

In conclusion, velocity measurements gave the opportunity to verify the proposed mean velocity field defined by $U=U_{0} e^{-\nu x}$ in Eq. (2.17), and they were generally needed for analytical or numerical modeling. Salinity, on the other hand, if used as proxy, defined with Eq. (4.1), gives an innovative and potentially useful substitution for obtaining conservative solute concentration statistics with an emphasis on the requirement of a steady and stratified state of the estuary.

\subsection{Numerical modeling}

Since velocity measurements were only input data for the analytical or the numerical model, the first idea for testing the methodologies was to create an independent numerical model which could simulate the problem. This approach is presented in Section 4.3.1, and its results along with a comparison can be found in Chapter 5. Later on, after the salinity measurements had been taken within the CPoRT project, and a more complex model (MOHID) had become available, the next idea consisted of simulating the Žrnovnica hydrodynamic system in MOHID. The Žrnovnica model in MOHID is presented in Section 4.3.2, while the 

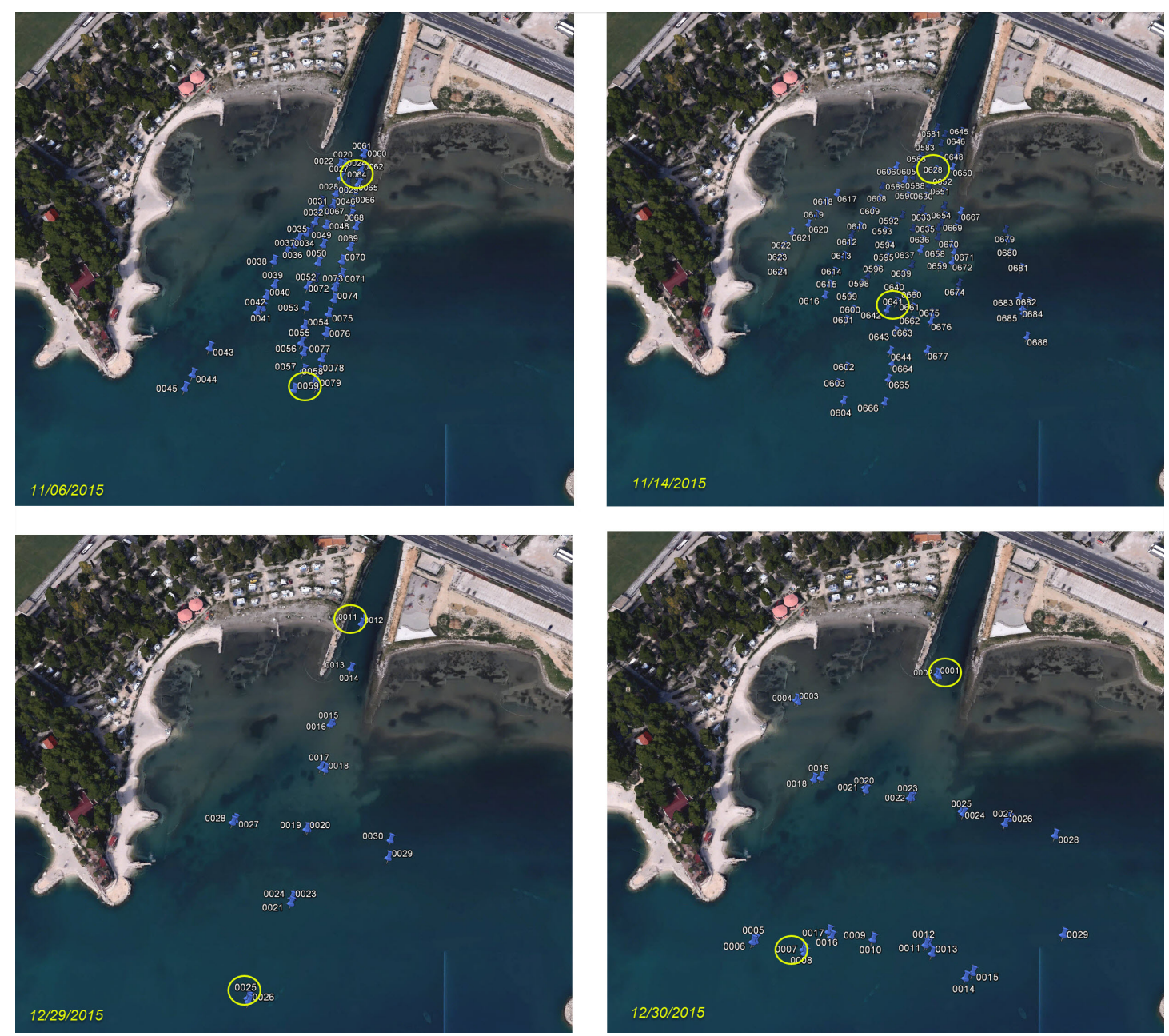

FIGURE 4.10: Recorded positions for different salinity measurement campaigns with indicated dates and points represented in the results.

results and comparisons are correspondingly given in Chapter 5.

\subsubsection{Random walk particle tracking approach}

Random walk particle tracking (RWPT) models are typical examples of the Lagrangian approach, which became an alternative to Eulerian transport models due to their common issues of numerical dispersion and artificial oscillations, especially for advective-dominated problems. Lagrangian treats the transport of solute mass using a large number of particles, whereby each particle is observed separately (Fig. 4.11) and the concentration is calculated by summing up the particles at a certain location. Such an approach allowed the RWPT model to avoid 


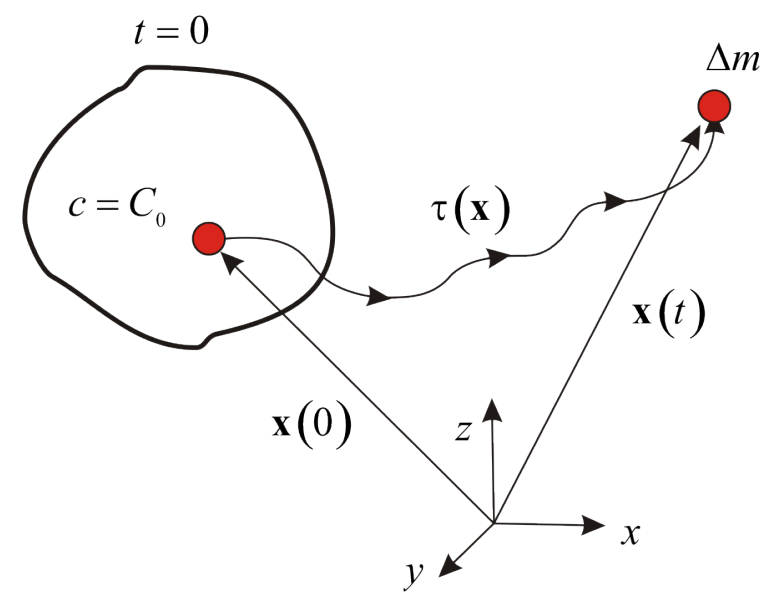

FIGURE 4.11: General random walk particle tracking scheme.

solving the transport equation directly, which results in an absence of numerical dispersion and also increases computational efficiency [118]. RWPT was frequently used in groundwater for modeling complex, high-resolution transport problems, along with inverse modeling and uncertainty assessment for contaminant transport (e.g. [145, 1, 51, 68, 135, 6, 137], and many others).

This approach was also used in coastal applications to display residual circulation [31], dispersion in shallow waters [77], and to simulate transport in vertically well-mixed estuaries and coastal waters [44]. Subsequently, it was used for modeling mixing effects in water bodies of coastal areas [145], for estimation of connectivity characteristics regarding the circulation fields [34], and, more recently, for evaluating the flushing potential in coastal zones [92].

In general, RWPT can be illustrated by Fig. 4.11, where one particle trajectory is followed and its displacements are driven by advection and diffusion. Each particle has a mass $(\Delta m)$, and its position at each time is defined by the space vector $\mathbf{x}(t)$, and $\tau(\mathbf{x})$ presents the traveling time for that particle. By multiplying that process by the number of particles used, the concentration at the chosen detection volume can be obtained:

$$
c(\mathbf{x}, t)=\frac{\sum_{i=1}^{N} \Delta m_{i} \cdot \omega_{i}}{V_{\operatorname{det}}}
$$

where $N$ equals the number of particles within the relevant detection volume $\left(V_{\mathrm{det}}\right), \Delta m_{i}$ represents the mass of a particle, and $\omega_{i}$ is the weighting coefficient for the level of involvement of each particle $i$. The concept of relevant detection volume is presented with a circle of a radius $r$ in Fig. 4.12, and the concentration for each cell is defined by Eq. (4.2). 


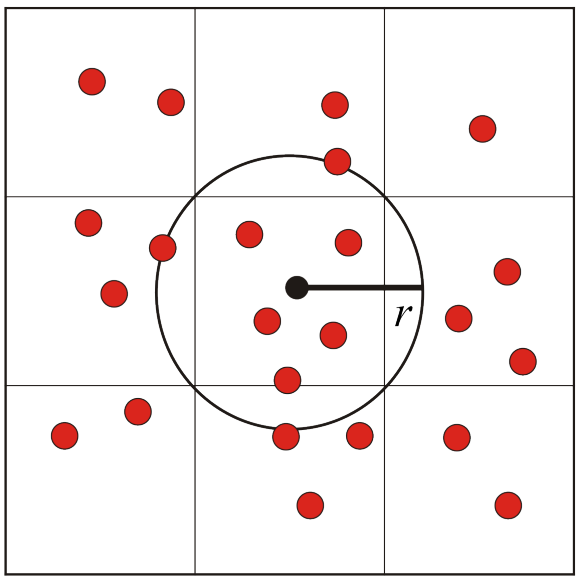

FIGURE 4.12: Concentration calculation in the RWPT model.

The numerical random walk particle tracking model used herein follows the approach developed by [57] for calculating groundwater age, yet in this case it is adjusted for surface riverine solute transport in the estuary in accordance with guidelines from [63]. The domain is set as a rectangle (30 $\mathrm{m} \times 100 \mathrm{~m})$ and discretized by 12,000 elements $(0.5 \mathrm{~m} \times 0.5 \mathrm{~m})$.

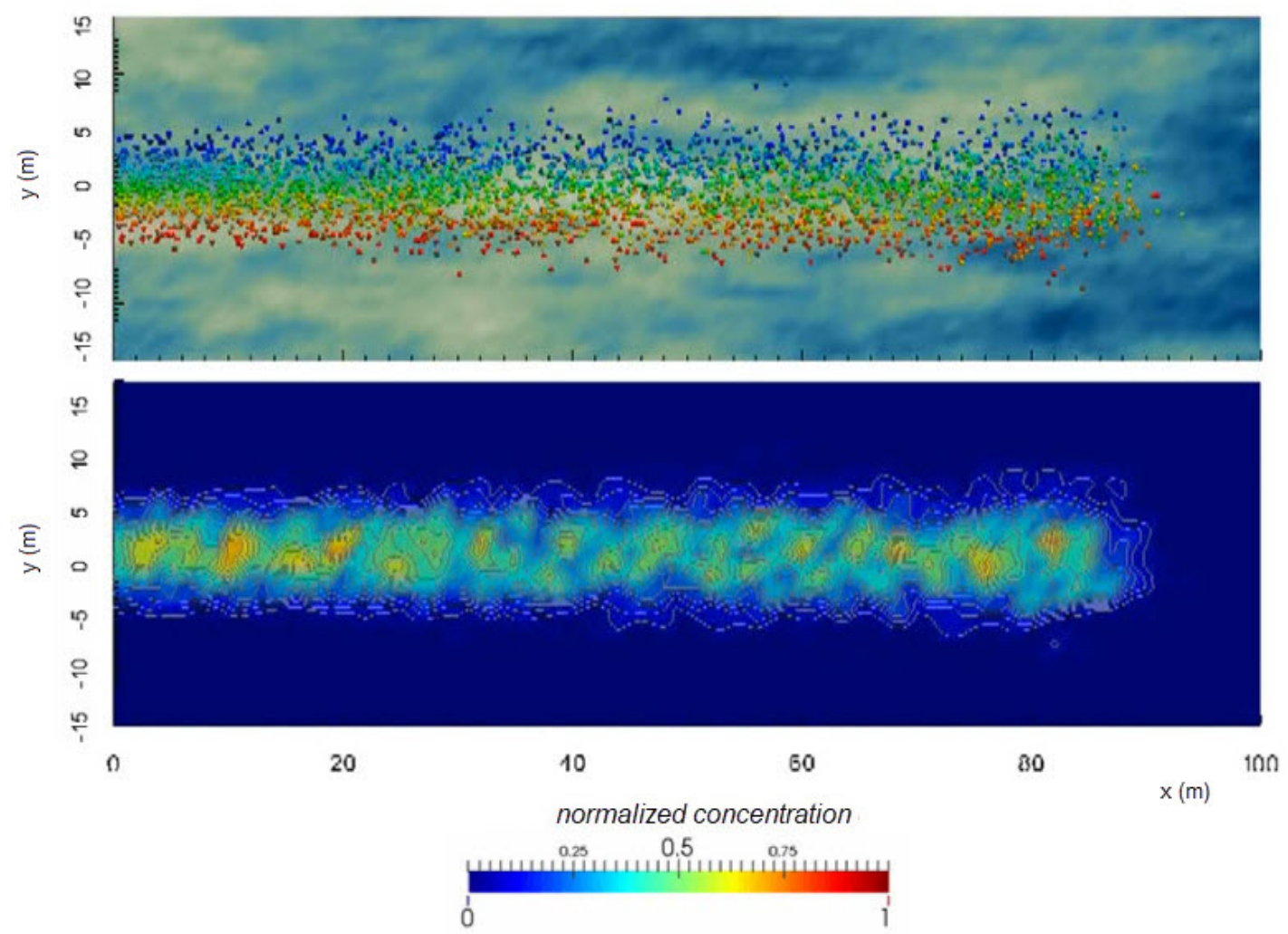

FIGURE 4.13: An example of a RWPT simulation. 
The model introduces a constant number of particles at the source (i.e. the river mouth) distributed following the Gaussian function. That is accomplished by setting the starting positions of particles at source ( $y$ axis) according to Gaussian distribution with the mean value set to 0 , and variance set to $\sigma_{y_{0}}^{2}$ or $(b / 6)^{2}$. To simulate a continuous release, we introduce 30 particles at each time step. The number of steps for each simulation is determined by the condition of reaching a steady state. In each step the particles occupy the position defined by a Gaussian probability distribution with parameters equivalent to those used in the analytical model. A steady state is reached when the difference between the number of particles entering and exiting the observed domain is smaller than $2 \%$ of those counted over the last ten time steps. Particles move in time increments $\Delta t$, and the position of each particle is defined as:

$$
\begin{aligned}
& x_{(t+\Delta t)}=x_{t}+u_{x_{t}} \Delta t+Z_{1} \sqrt{2 D \Delta t} \\
& y_{(t+\Delta t)}=y_{t}+Z_{2} \sqrt{2 D \Delta t}
\end{aligned}
$$

where $x_{t}$ and $y_{t}$ are the coordinates of the particle from the previous step, $u_{x_{t}}$ is local particle velocity at previous step $t, D$ is the dispersion coefficient, equivalent to the spatial variance growth rate $e_{t}$, and $Z_{1}, Z_{2}$ are random numbers generated from the Gaussian standard distribution. The numerical model uses $D=0.01$ $\mathrm{m}^{2} / \mathrm{s}$ assumed to be equivalent to $e_{t}$ defined in Gaussian spatial transversal concentration variance $\left(\sigma_{y}^{2}=2 e_{t} t+\sigma_{y_{0}}^{2}, \sigma_{y_{0}}=b / 6\right)$, a river mouth width $b=14 \mathrm{~m}$, and a time step of $1 \mathrm{~s}$. An example of the simulation done by this RWPT model is given in Fig. 4.13.

For each element, the concentration is calculated as a sum of particles using weighting coefficients calculated by a modified Gaussian Kernel smoother [54]. Each weighting coefficient $\omega_{i}$ was calculated by:

$$
\omega_{i}=e^{-\frac{d_{i}^{2}}{2 l^{2}}}
$$

where $d_{i}$ represents the absolute distance of each (i) particle from the center of the cell, and $l$ is the length scale for the input space. In order to reduce the computational time, the length scale $l$, was defined by limiting the entire input space to the detection volume defined by a circle of a radius $r$ (see Fig. 4.12), such that $\omega_{i}$ for $d=r$ is set to 0.001 resulting in $l=0.19 \mathrm{~m}$. As expected, the highest contributing weighting coefficient $\omega_{i}=1$ is obtained for $d=0$, which can be seen in Eq. (4.4).

Convergence tests with regard to the number of particles used in each step and the number of performed realizations in order to obtain the first two moments 
are shown in Chapter 5 (Fig. 5.33). The selected number of particles was 30 and the number of realizations was 1000. Normalized concentration in each cell $\left(c_{i}\right)$ was calculated by:

$$
c_{i}=\frac{N_{i}}{C_{0}}
$$

where $N_{i}$ is the number of summed up weighted particles within the detection volume defined by $r$ (see Fig. 4.12), and $C_{0}$ is defined as maximum mean sum in all 1000 realizations:

$$
C_{0}=\max \left(\overline{N_{i}}\right), \quad \overline{N_{i}}=\frac{\sum N_{i}}{1000}
$$

The mean and variance were obtained using the Eq. (4.6) for calculating cell concentrations in each of the 1000 realizations. Finally, results are given and compared to the proposed analytical model in Chapter 5.

\subsubsection{Complex modeling approach - MOHID}

In the course of the CPoRT project, access was granted to a more sophisticated and complex model. The model of the Žrnovnica was created accordingly during the research conducted at MARETEC (Marine Environment and Technology Center) in Lisbon, Portugal.

\section{MOHID Water Modeling System background}

MOHID or Modelo hidrodinâmico is a three-dimensional water modeling system developed by MARETEC (Marine Environment and Technology Center) at Instituto Superior Técnico (IST) which is part of the Universidade de Lisboa in Portugal [97]. It was initially developed as a 2D hydrodynamic model to be used for studying estuaries and coastal areas by the implementation of a finite difference approach [109]. During the recent several decades, the model has evolved to an object-oriented water modeling system, programmed in ANSI FORTRAN 95 and comprising over 40 modules which consist of more than 240,000 lines of code. The present version of MOHID (it is continuously upgraded) is a modeling system, based on a finite volume approach, which allows the adoption of an integrated modeling philosophy, not only of processes (physical and biochemical), but also of different scales (allowing the use of nested models) and systems (estuaries and watersheds). So far, MOHID has been applied to different case studies, coastal and estuarine areas, and oceanic processes and reservoirs, since it is able 
to simulate the complex properties of the flows [95].

The main features of MOHID are [97]:

- Possibility to run different applications simultaneously

- Easily adaptable to new application areas

- Spatial discretization using the finite volume approach technique (the spatial coordinates are independent and any geometry can be chosen for every dimension), allows for more flexibility in the subdivision of the vertical and horizontal domains

- Enables a model to be used in 1D, 2D, or 3D

- It can use different time steps for different modules

- The nested models may be used and are only limited by the available computational power

- Possibility of parallel execution of different models on separate processors, communicating between themselves using the MPI protocol (Message Passing Interface), thus allowing a very meaningful gain in calculating time

MOHID has three main different tools: MOHID Water, MOHID Land and MOHID Soil. These tools are based on the same framework and can therefore be coupled and used to study the water cycle in an integrated approach. For the purpose of comparing the results from the analytical methodology developed in this thesis, velocity and salinity measurements, and numerical modeling, the MOHID Water tool was used. MOHID Water is a three-dimensional numerical program for simulating surface water bodies including oceans, estuaries, and reservoirs. It has already been applied to several coastal and estuarine environments in Portugal, Spain, France, Brazil, and India (e.g. [119, 149, 66, 40, 43, 110]). The framework of the modeling system is illustrated in Fig. 4.14 and the key scientific areas covered by MOHID Water are listed below:

- Hydrodynamics - covers the calculation of circulation patterns and velocity fields that transport the water properties, which are available in the model (sediments, phytoplankton, nitrate, arsenic, ammonia, etc.)

- Waves (the impacts of wave refraction and diffraction on the sediment)

- Sediment dynamics (vertical and horizontal transport)

- Water quality (modeling of nutrient cycles) 


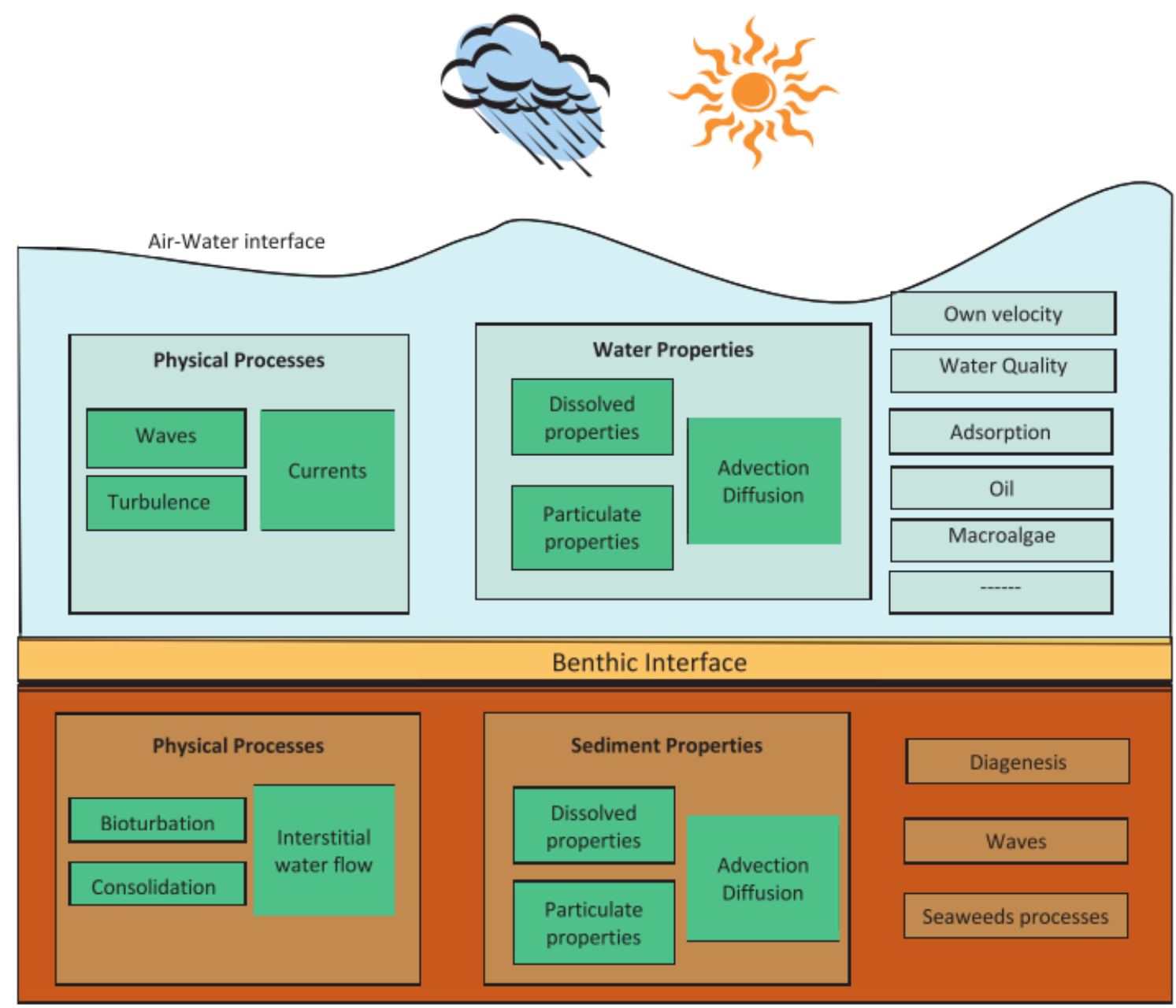

FIGURE 4.14: Schematic representation of the MOHID Water framework (Courtesy of [95]).

- Oil spills

- Jet modeling

- Modeling of turbulent mass, heat, and momentum transport

The above-listed processes are simulated using modules that are included in the simulation by a user, according to a user's modeling requirements. MOHID Water has numerous different modules, but the ones that cover water physics [98] are:

- Hydrodynamic - the basic required module that solves primitive continuity and momentum equations for surface elevation and the 3D velocity field for incompressible flows, in orthogonal horizontal coordinates and generic vertical coordinates, assuming hydrostatic equilibrium and Boussinesq approximations. 
- AdvectionDiffusion - defines the relations and behavior of both the advection and the diffusion transport process.

- WaterProperties - responsible for computing the properties evolution in the water column, including temperature, salinity, cohesive sediments, phytoplankton, nutrients, contaminants, etc., depending on other modules involved, such as AdvectionDiffusion and WaterQuality

- Turbulence - calculates the turbulent transport of mass, heat, and momentum.

- InterfaceWaterAir - defines the conditions at the water-air boundary by implementing air-water fluxes (they can be specified by the meteorological model or computed using air temperature, moisture, wind speed, cloud cover, and solar radiation and coefficients provided by the user)

- InterfaceSedimentWater - defines the conditions at the bottom-water boundary by computing the fluxes, but is also the owner of state variables having negligible mobility (filter feeders, microphytobenthos, decomposers, etc.)

- Waves - calculates the impact of the waves

- Lagrangian - includes tracking for a set of chosen particles

- Lagrangian Global - also includes tracking for a set of chosen particles, but for the global model in case the user requires a nested model

Among the many different modules, MOHID Water Modeling System also possesses a large variety of support tools which can help create simulations, prepare (preprocess) and process input and output data files.

As explained by [7, 95], in general, MOHID is based on an integral approach which equals the rate of accumulation inside a control volume to the sum of input and output fluxes, plus sources minus sinks:

$$
\frac{\partial}{\partial t}\left(\iiint_{C V} \beta d V\right)=-\iint_{\text {surface }}[(\beta \vec{u} \cdot \vec{n})+(-\vartheta \vec{\nabla} \beta \cdot \vec{n})] d A+\iiint_{C V}\left(S_{0}-S_{I}\right) d V
$$

where $C V$ is the Control Volume, $u$ is the fluid velocity relative to the surface $A, \vartheta$ is the diffusivity, $\beta$ is a generic water property (such as phytoplankton and nutrients), and $S_{O}-S_{I}$ is the difference between sources and sinks of the property $\beta$ per unit volume. If Gauss's theorem is applied to Eq. (4.7), the differential 
conservation equation is defined by:

$$
\frac{\partial \beta}{\partial t}=-\frac{\partial}{\partial x_{j}}\left(u_{j} \beta-\vartheta \frac{\partial \beta}{\partial x_{j}}\right)+\left(S_{0}-S_{I}\right)
$$

which states that the rate of change for an arbitrary property in a point balances the divergence of the advection plus diffusion fluxes plus the production minus the consumption rate per unit of volume. This can further be written in Lagrangian reference where the time derivative states that the rate of change in an elementary volume of fluid balances diffusion across its boundaries plus sources minus sinks:

$$
\frac{d \beta}{d t}=\frac{\partial}{\partial x_{j}}\left(\vartheta \frac{\partial \beta}{\partial x_{j}}\right)+\left(S_{0}-S_{I}\right)
$$

This equation is obtained if the surface of the control volume from Eq. (4.7) is moving at the fluid velocity, which is then a foundation for the Lagrangian module to compute dilution through a volume increasing rate, as a result of the entrainment of the surrounding fluid. The change of concentration for property $\beta$ is obtained as the difference between the concentration inside the volume and the concentration of the entrained fluid.

The velocity vector $\left(\vec{u}(\mathbf{x})=\left[u_{x}, u_{y}, u_{z}\right]\right)$ used in Eq. (4.7) and Eq. (4.8) are obtained by solving the 3-D incompressible primitive equations where the assumptions of hydrostatic equilibrium and Boussinesq approximation are applied to Reynolds averaged Navier-Stokes equations (RANS). Therefore, the momentum balance equations for mean flow horizontal velocities are given in the next form [116]:

$$
\begin{aligned}
\frac{\partial u_{x}}{\partial t} & =-\frac{\partial\left(u_{x} u_{x}\right)}{\partial x}-\frac{\partial\left(u_{x} u_{y}\right)}{\partial y}-\frac{\partial\left(u_{x} u_{z}\right)}{\partial z}+f u_{y} \\
& -\frac{1}{\rho_{0}} \frac{\partial p_{a t m}}{\partial x}-g \frac{\rho(\eta)}{\rho_{0}} \frac{\partial \eta}{\partial x}-\frac{g}{\rho_{0}} \int_{z}^{\eta} \frac{\partial p^{\prime}}{\partial x} d z \\
& +\frac{\partial}{\partial x}\left(A_{H} \frac{\partial u_{x}}{\partial x}\right)+\frac{\partial}{\partial y}\left(A_{H} \frac{\partial u_{x}}{\partial y}\right)+\frac{\partial}{\partial z}\left(A_{V} \frac{\partial u_{x}}{\partial z}\right) \\
\frac{\partial u_{y}}{\partial t} & =-\frac{\partial\left(u_{x} u_{y}\right)}{\partial x}-\frac{\partial\left(u_{y} u_{y}\right)}{\partial y}-\frac{\partial\left(u_{z} u_{y}\right)}{\partial z}+f u_{x} \\
& -\frac{1}{\rho_{0}} \frac{\partial p_{a t m}}{\partial y}-g \frac{\rho(\eta)}{\rho_{0}} \frac{\partial \eta}{\partial y}-\frac{g}{\rho_{0}} \int_{z}^{\eta} \frac{\partial p^{\prime}}{\partial y} d z \\
& +\frac{\partial}{\partial x}\left(A_{H} \frac{\partial u_{y}}{\partial x}\right)+\frac{\partial}{\partial y}\left(A_{H} \frac{\partial u_{y}}{\partial y}\right)+\frac{\partial}{\partial z}\left(A_{V} \frac{\partial u_{y}}{\partial z}\right)
\end{aligned}
$$


Where $u_{x}, u_{y}$ and $u_{z}$ are the components of the velocity vector in the $x, y$ and $z$ directions respectively, $f$ is the Coriolis parameter, $A_{H}$ and $A_{V}$ are the eddy viscosities in the horizontal and vertical directions and $p_{a t m}$ is the atmospheric pressure. Furthermore, $\rho$ is the specific mass, $\rho^{\prime}$ is the specific mass anomaly and $\rho_{0}$ is the reference density $\left(\rho=\rho_{0}+\rho^{\prime}\right)$, while $\rho(\eta)$ represents the density at the free surface. The temporal evolution of velocities (term on the left hand side) is the balance of advective transports (first three terms on the right hand side), Coriolis force (forth term), pressure gradient (next three terms) and turbulent diffusion (last three terms). The vertical velocity $\left(u_{z}\right)$ is then calculated from the incompressible continuity equation (mass balance equation):

$$
\frac{\partial u_{x}}{\partial x}+\frac{\partial u_{y}}{\partial y}+\frac{\partial u_{z}}{\partial z}=0
$$

by integrating between bottom $(-h)$ and the depth $z$ :

$$
u_{z}=\frac{\partial}{\partial x} \int_{-h}^{z} u_{x} d x+\frac{\partial}{\partial y} \int_{-h}^{z} u_{y} d y
$$

And the free surface equation is obtained by integrating the equation of continuity over the whole water column (between the free surface elevation $\eta(x, y)$ and the bottom):

$$
\frac{\partial \eta}{\partial t}=-\frac{\partial}{\partial x} \int_{-h}^{\eta} u_{x} d z-\frac{\partial}{\partial y} \int_{-h}^{\eta} u_{y} d z
$$

The hydrostatic equilibrium $\left(\frac{\partial p}{\partial z}+g \rho=0\right)$ with above references is introduced in both Eq. (4.10) and Eq. (4.11) (three pressure terms on the right side of equation) from:

$$
p(z)=p_{a t m}+\rho_{0} g(\eta-z)+g \int_{z}^{\eta} p^{\prime} d z
$$

Eq. (4.15) relates pressure at any depth with the atmospheric pressure at the sea surface, the sea level and the anomalous pressure integrated between that level and the surface.

There are numerous possibilities of simulating using the MOHID systems, specifically MOHID Water for the purposes of this research, and coupling with other modules if a more integrated approach is required. All modules communicate with each other if called properly within, and if the right syntax is adhered 
to. In general, MOHID's code is open source; however, its usage is made significantly easier by using the graphical user interface, namely MOHID Studio, which requires a license from ActionModulers [2], which is a spin-off company originating from MARETEC and continuously working with them [50]. However, the license for academic usage is available for free upon request, hence the application being used in this work.

\section{MOHID setup for the Žrnovnica estuary}

For the case of Žrnovnica estuary only the MOHID Water tool is used with an emphasis on Hydrodynamic and WaterProperties modules, using the required accompanying ones, including InterfaceWaterAir, InterfaceSedimentWater, Turbulence, etc. Although the sources for input data were previously described in Section 4.1, some specifics required for the model of the Žrnovnica estuary are presented here. The basic data necessary for the geometry setup consists of bathymetry (see Fig. 4.2) and coastline obtained by geodetic surveying (see Fig. 4.15).

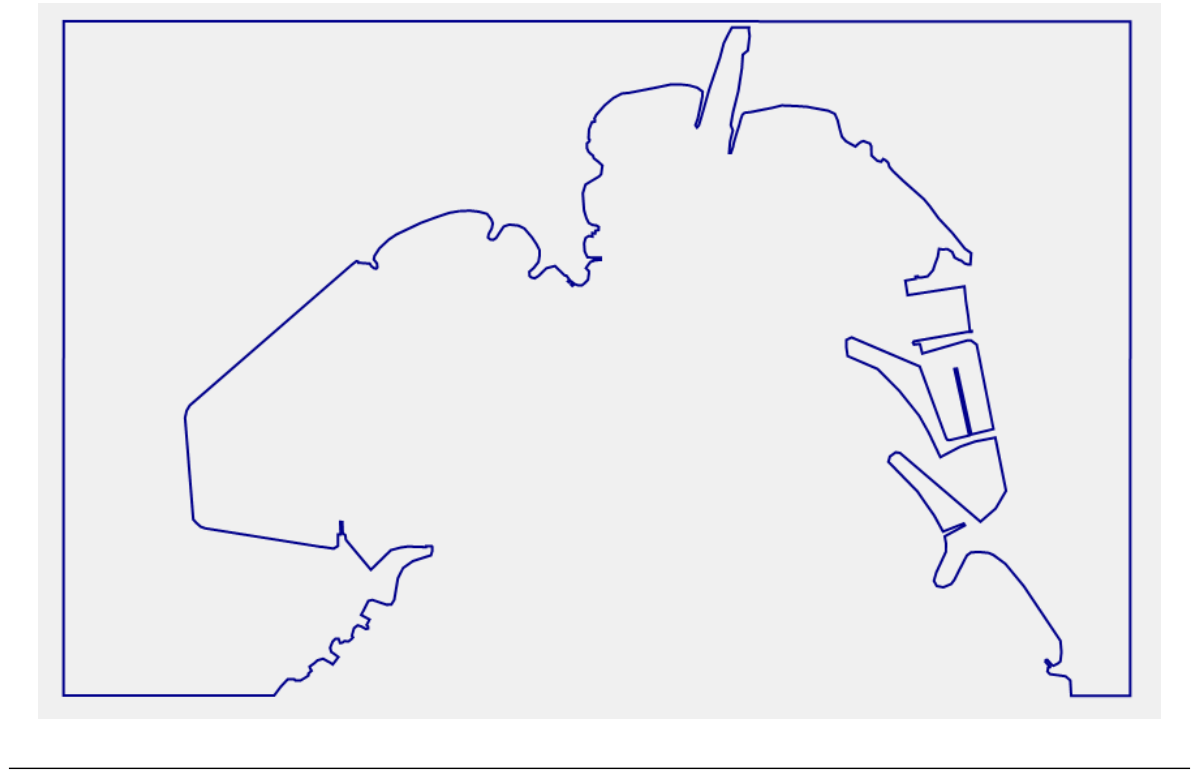

FIGURE 4.15: Coastline for the case study of the Žrnovnica estuary obtained by geodetic surveying.

The rest of the input data depends on the level and characteristics of conducted simulations. In this case, wind data, tidal data, and flow input are included. Wind and flow data had to to be adjusted in specific input files as per MOHID requirements. The tidal information can be applied by using the finite elements solution (FES) [93] and in this case FES2004 was used, which is already 


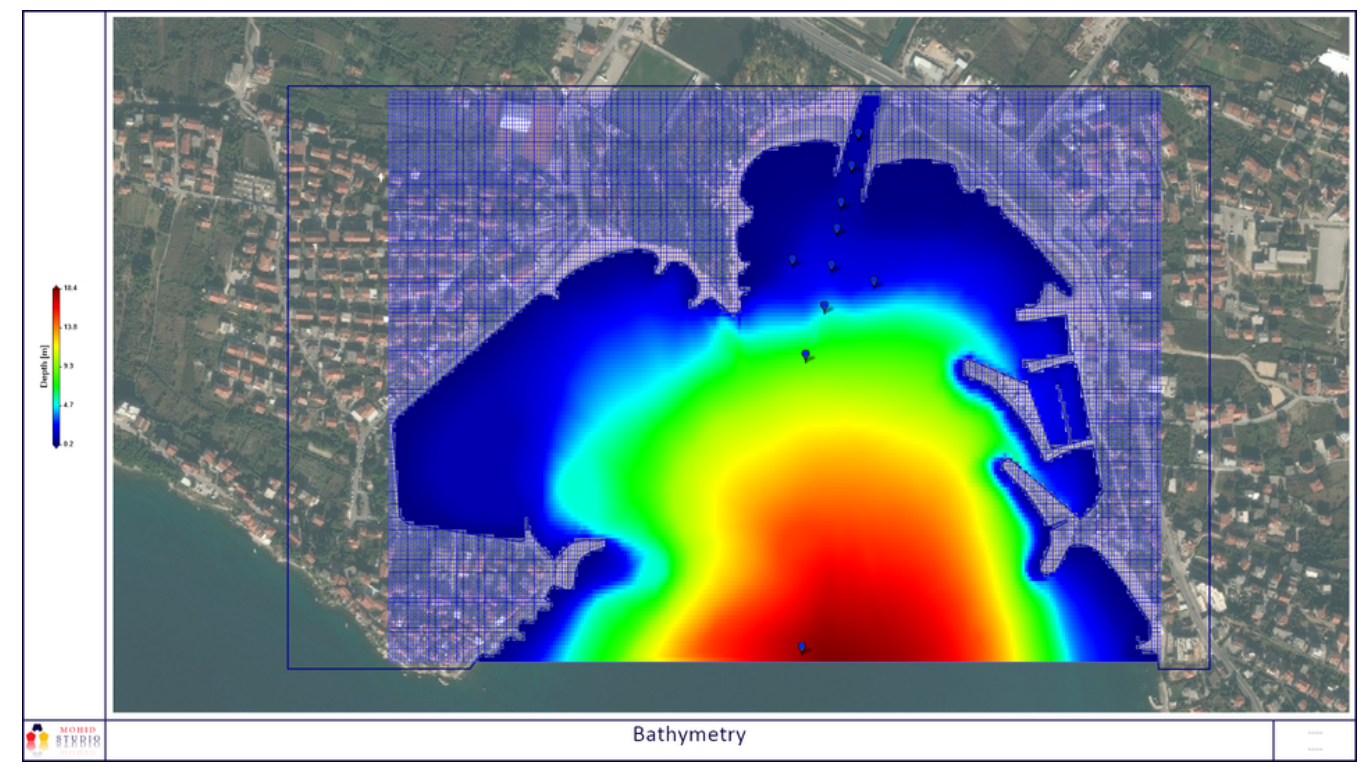

FIGURE 4.16: Bathymetry and defined grid for the Žrnovnica estuary in MOHID.

implemented in the MOHID user interface as a preprocessing tool. By investigating the possibilities of MOHID for the purposes of this research, two different variations of MOHID models for the Žrnovnica estuary were developed in the CPoRT project, one in 2D and the other one in 3D. Since it was a progression of ideas, firstly a basic 2D hydrodynamic model was developed by including wind, tidal data, and setup of the river flow which was discretized in accordance with a global numerical grid. The 10 minutes' wind data [111] were linearly interpolated to match the time step of the simulation which was set to $5 \mathrm{~s}$. The wind stresses were then calculated within the model itself. The whole process included the following procedures:

- Adjusting the bathymetry by choosing adequate cell size and then smoothing if necessary (smoothing is enabled as a MOHID Studio tool). The final bathymetry which is geometrically limited by the coastline, along with the implemented grid ( $3.5 \mathrm{~m} \times 3.5 \mathrm{~m})$, is shown in Fig. 4.16.

- Setting one control point at the most open border of the grid (the southernmost point in Fig. 4.16), which was used to compare the time series of water levels calculated by the model (using FES2004) to those measured at the station of IOF [111] nearby.

- Setting up the properties in InterfaceSedimentWater and InterfaceWaterAir 
modules which define the boundary conditions (see Fig. 4.14). In both modules, rugosity was set with constant and model default values. For the sediment-water boundary this was justified due to a predominately continuous and smooth sea bottom, while the assumptions of a steady state with small amplitudes in water level (due to tides and wind-generated waves) justified the constant water-air boundary conditions.

- The WaterProperties module was needed in order to make a corresponding comparison with salinity measurements, and for the verification of results from the analytical model. However, the initial water properties values (before the river flow is entered into simulation), namely salinity (38 PSU, PSU $\cong \% \mathrm{~g} \cong \mathrm{gg})$ and temperature $\left(18^{\circ} \mathrm{C}\right)$ were set to those measured in the field.

- The additional points that can be seen in Fig. 4.16 represent the points of measurements from the campaign on December 29, 2015, as visible in Fig. 4.10. These points are used to compare the velocities obtained by the proposed analytical model (Eq. (2.17)), measured data, and the mean values of velocities in those same points for the MOHID hydrodynamic simulation at the time of the measuring campaign. These results are provided in Chapter 5.

- The Turbulence module defines viscosity as constant in the horizontal domain, while vertical viscosity is not used for the 2D model (no vertical movement). This viscosity represents a mixture or diffusion associated to processes not taken into account in cell average velocity (called turbulence), and so it depends on the mesh used, the units are $\mathrm{m}^{2} / \mathrm{s}$ and is defined as velocity $(\mathrm{m} / \mathrm{s})$ multiplied by mixture length $(\mathrm{m})$, i.e. the size of the cell. If cells are relatively small in comparison to the entire domain, the viscosity may be assumed to be $10 \%$ of the grid size.

The general hydrodynamic model was set to run for a period of three days starting on December 27 at midnight until December 29 at midnight. The initial two days were given in order to stabilize the model so that on the $29^{\text {th }}$, when the measurements were taken, a steady state could be achieved as much as possible.

As opposed to the 2D model, there are some key differences when implementing a 3D model:

- In the geometry module, what represented a single layer in a 2D model is now a set of a user-defined number layers with different types of discretization available (sigma, cartesian, or combined domain). In this case, 


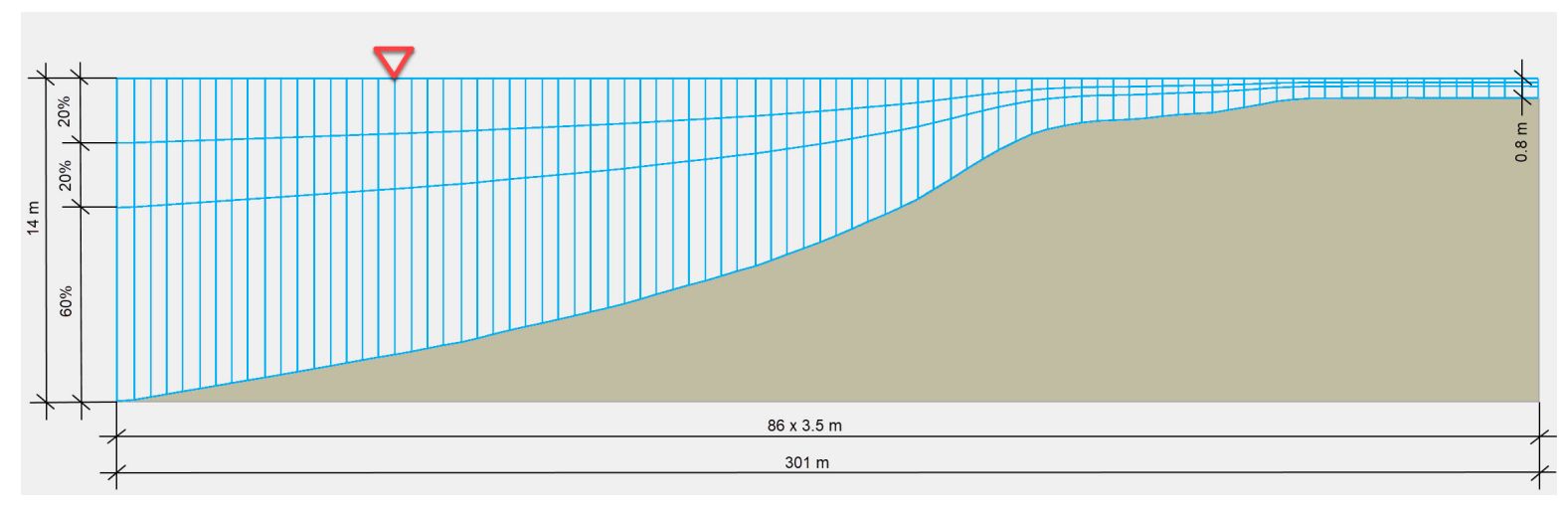

FIGURE 4.17: An example of vertical discretization for the MOHID $3 \mathrm{D}$ model given by a vertical cut in the $\mathrm{Y}-\mathrm{Z}$ plane.

the sigma domain was preferable and an example of a vertical cut ( $\mathrm{Y}-\mathrm{Z}$ plain, a length of $300 \mathrm{~m}$, starting from the middle of the river mouth) showing the defined discretization is given in Fig. 4.17.

- When defining discharge for simulating river inflow, the source is distributed over the horizontal discretization in the top layer of the water column.

- The Turbulence module defined by previously explained constant viscosities is not enough anymore and thus the 3D model requires a GOTM (General Ocean Turbulence Model) module for simulating vertical turbulence. GOTM is a one-dimensional water column model for studying hydrodynamic and biogeochemical processes in marine and limnic waters [8].

For the purpose of the Žrnovnica estuary simulation, within the GOTM module, a turbulence model which includes calculation of the total kinetic energy, length scale, and stability functions was used. Total kinetic energy is obtained by a dynamic equation for the $k-\varepsilon$ model [88], length scale was defined by a dynamic dissipation rate equation, and stability functions are obtained according to [20]. These options vary from case to case, and the rest of the properties in the GOTM module are mostly default settings. Nevertheless, if a special case occurs, they can be changed. Since MOHID is open-source, one may model their own turbulence settings. GOTM, however, covers most options and it is under continuous development by numerous users worldwide.

In both cases, the Lagrangian module was included, with 10 particles entering the system each $10 \mathrm{~s}$, starting at the river mouth $(20 \mathrm{~cm}$ deep). The particles were introduced into the system at the start of December 29, after a hydrodynamic steady state had been achieved. Since the idea with MOHID was to simulate the behavior of a proxy substance, i.e. salinity, only the salinity and temperature 
properties were set for Lagrangian particles corresponding to freshwater properties (2.0 PSU, and $\left.14^{\circ} \mathrm{C}\right)$. Particle movement within the module is modeled after random walk particle tracking methodologies defined by [138] and [3]. However, when the Lagrangian module was included, the calculation took two and a half days to complete for the $2 \mathrm{D}$ model, and approximately five days for the $3 \mathrm{D}$ model, on a standard desktop PC (Intel i5 processor, 8 GB of RAM). Different approaches were then considered, including the implementation of a nested model where the global (bigger) grid would solve the tides and wind stress effects, but then it would be transferred to nested one (smaller and closer area to the river mouth) with a denser grid. However, it has demonstrated many problems when defining the boundary conditions between the main model and the smaller one (daughter grid), especially when the Lagrangian module was included. Hence, in general, for the Stobreč Bay area, a single-grid model (without nesting) was considered adequate.

To conclude with the MOHID research, both the 2D and the $3 \mathrm{D}$ model have shown interesting aspects of this potential approach to verification. However, there are some specific differences which will explain the results in Chapter 5. For instance, the 2D model cannot simulate the stratification that is present in the estuary, since it only recognizes different depths as different permeability for the water flow. In such a case, bathymetry is expected to have much more influence that it should. The 2D model is simpler and enables easier implementation of the Lagrangian module. Nevertheless, the 3D model will produce more realistic results since it will manifest stratification due to vertical discretization (see Fig. 4.17) and decrease the influence of bathymetry. Furthermore, by including vertical mixing and stratification, the river flow becomes more dominant as it slides over the denser sea wedge. These features are all exhibited in the MOHID results in Chapter 5.

While conducting research for this thesis, different approaches to verification were considered. The final concept, as can be witnessed throughout Chapter 4 , is a somewhat mixed system of measurements and modeling which has gradually evolved. Nevertheless, there are still ideas and potential actions to be undertaken in both measurements and modeling in order to achieve better verification. Some of those are mentioned in Chapter 7. 


\section{Chapter 5}

\section{Results}

This chapter presents the results of all the research conducted during the development of the thesis. Since the research process was organized using two main analytical methodologies (Chapter 2 and Chapter 3), followed by field measurements and numerical modeling (Chapter 4), the results follow the same order. The first two sections will demonstrate illustrative examples for using the developed analytical methodologies for point concentration statistics in Section 5.1 and for spatially integrated concentration statistics in Section 5.2. Key examples of field measurements and numerical modeling are shown in Section 5.3 , while in Section 5.4 the most important comparisons are given and discussed.

\subsection{Analytical modeling}

All details regarding the analytical modeling applied to this thesis are presented in Chapter 2 and Chapter 3. Illustrative examples used for the demonstration of the developed methodologies are mostly based on the conditions for the case study estuary, which is the Žrnovnica River estuary. In order to emphasize key influential processes, such as molecular diffusion and mean velocity attenuation, various illustrative examples are provided.

For the development of analytical, point, and spatially integrated statistics in the near- field zone of an estuary for a steady source in a steady flow, the mean concentration on a plane normal to the mean flow direction is assumed to follow a Gaussian one-dimensional distribution as described in Chapter 3 and defined by Eq. (3.10). Fig. 5.1 shows an example of implemented Gaussian distribution for unit steady mass loading $(L)$ of the solute through a plane normal to the flow direction $x$. The initial velocity used in this example is $0.4 \mathrm{~m} / \mathrm{s}$ and the attenuation coefficient $(\nu)$ is 0.0 . These values are part of the spatial variance defined by $\sigma_{y}^{2}=2 e_{t} t+\sigma_{y_{0}}^{2}, x=U t$, where $e_{t}$ is the constant spatial variance growth rate of $10^{-2} \mathrm{~m}^{2} / \mathrm{s}$. The initial value of $\sigma_{y_{0}}$ was set to $b / 6$, where $b$ is $10 \mathrm{~m}$. 


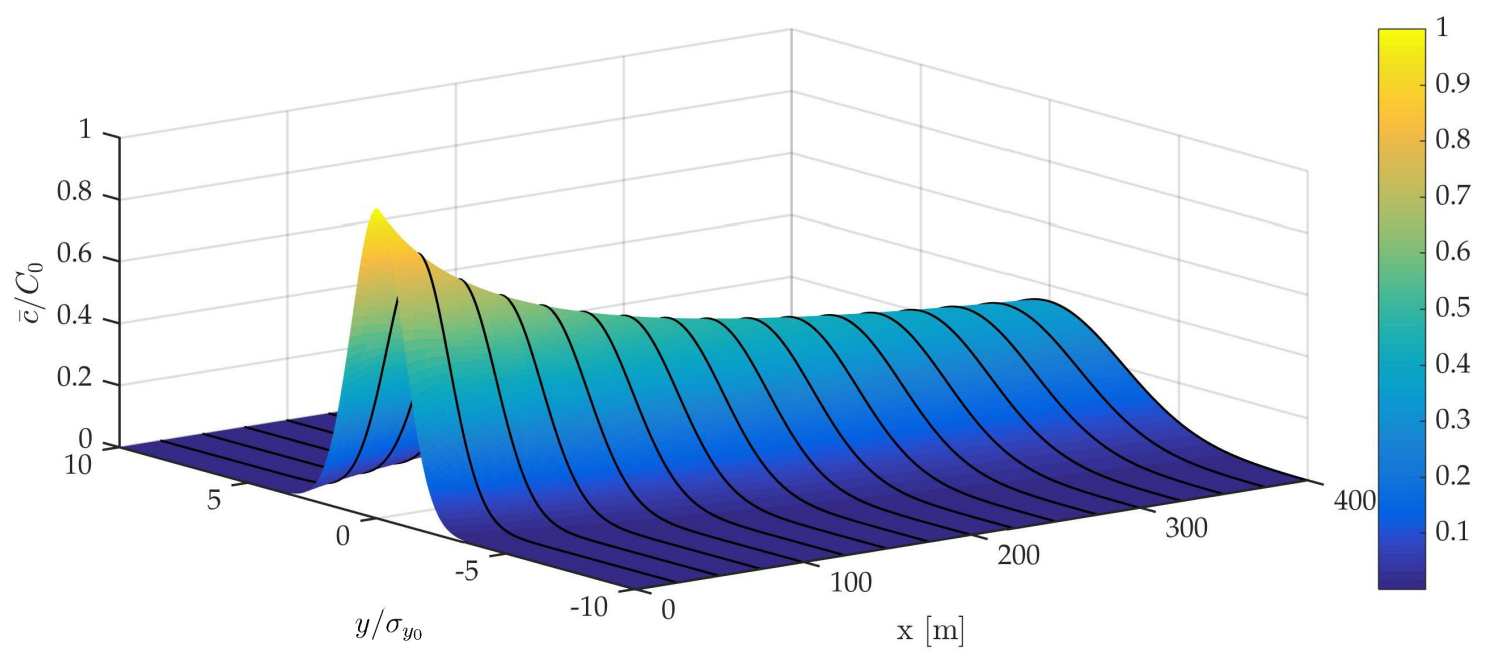

FIGURE 5.1: Gaussian one-dimensional distribution of mean concentration.

The mean distribution will subsequently be used as input data for the analytical models, but for different parameters, including either a steady mass flux, molecular diffusion, or velocity attenuation. However, the mean, by its very definition (Eq. (3.10)) is not sensitive to molecular diffusion, and even the effect of velocity attenuation is rather small in the near-field area and not easy to notice on the surface plot, which is used in Fig. 5.1. Hence, the effect of decreasing velocity is going to be presented through several transversal profiles of concentration mean below.

\subsubsection{Single point concentration statistics}

The first illustrative example shows the obtained concentration central moments for arbitrary conditions similar to those explained above (Fig. 5.1). However, no velocity attenuation is included here. The constant mean velocity is set to $0.4 \mathrm{~m} / \mathrm{s}$, and it is later used as the initial mean velocity value $\left(U_{0}\right)$. Molecular diffusion is rather small for surface water flow, but nevertheless present with the value of $10^{-9} \mathrm{~m}^{2} / \mathrm{s}$. In order to observe the downstream evolution of moments, several transversal profiles are selected and presented in Fig. 5.2.

An expected behavior can be observed in the mean, but also, more importantly, in other moments starting with variance $\left(\mu_{2}\right)$, whose initial bimodal nature is transformed into a unimodal distribution. For profiles close to the source (e.g. for $x=5,10,100 \mathrm{~m}$ ), variance exhibits lower values at $y / \sigma_{y_{0}}=0$ due to the zero mean gradient of the defined Gaussian distribution at source. Thus, the highest 

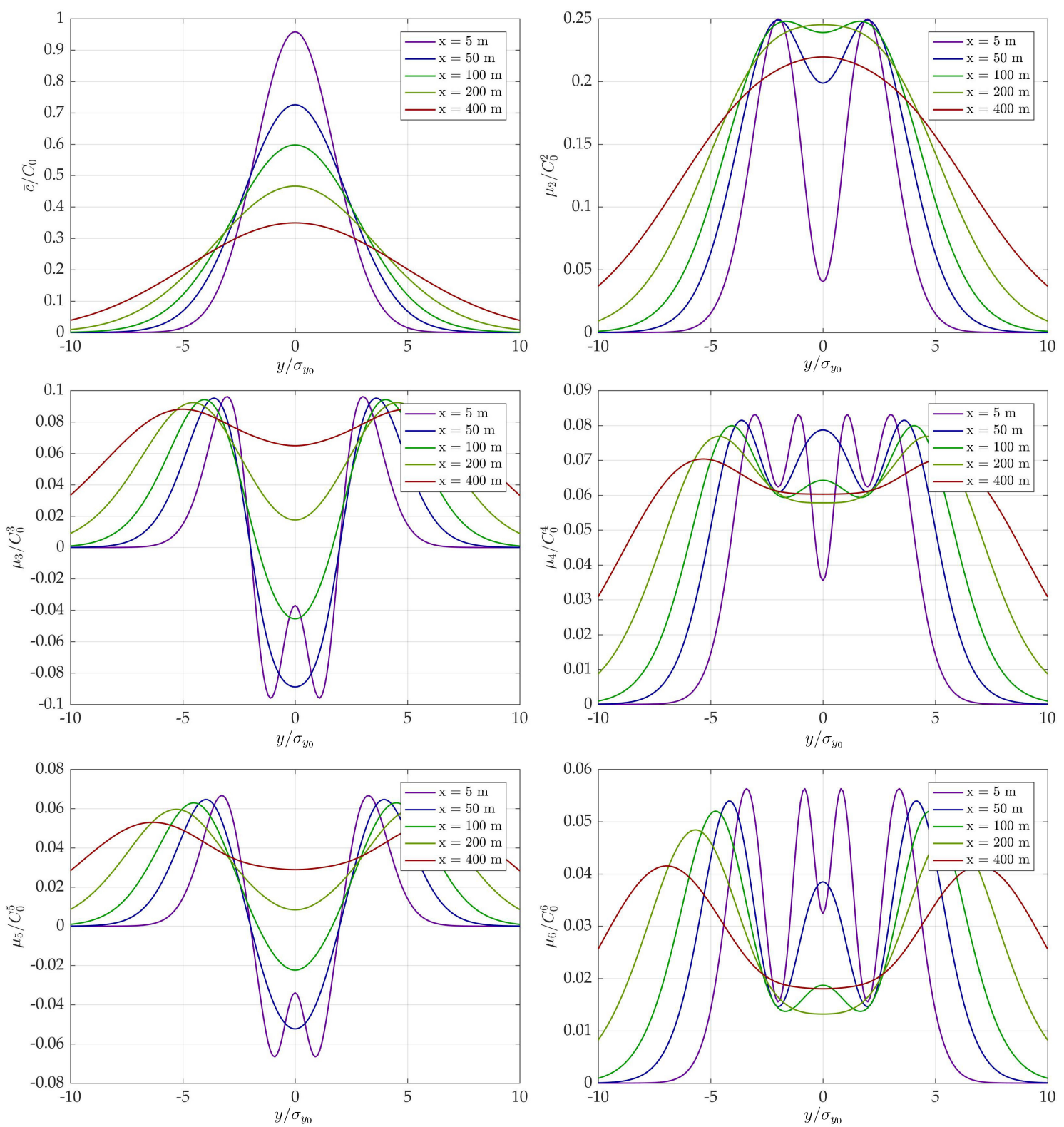

FIGURE 5.2: Illustrative example of normalized concentration mean and corresponding central moments $\mu_{2}, \mu_{3}, \mu_{4}, \mu_{5}$, and $\mu_{6}$ at different distances from the source.

values of variance are present at $y / \sigma_{y_{0}}=2.0$, following the largest mean gradient. The initial bimodal shape of variance is also affected by the source size whose impact is, as expected, stronger in the close profiles. For instance, if the source size (the width of the river mouth), were significantly smaller, the variance would be unimodal from the beginning, since the mean distribution would be narrower, and the higher gradient would be around $y / \sigma_{y_{0}}=0$. As one goes downstream, these extremes are diminishing due to a more emphasized effect of 
physical processes, particularly molecular diffusion, as investigated in the following paragraph (see Fig. 5.3). It was already discussed in Chapter 2 that the most influential indicator for PDF shape is variance $\left(\mu_{2}\right)$, since it defines the width and variability of the distribution around the mean. Higher central moments offer slightly more complex insight into the shape of the PDF. For instance, the third and the fourth one are used in a standardized form as skewness and kurtosis, which is in relative relationship to variance defined as:

$$
S=\frac{\mu_{3}}{\mu_{2}^{3 / 2}}
$$

and

$$
K=\frac{\mu_{4}}{\mu_{2}^{2}}
$$

Skewness and kurtosis offer physical meaning in the description of the PDF, where $S$ defines the degree of asymmetry of a distribution around its mean, while $K$ measures relative flatness of a distribution [133]. In Fig. 5.2, it can be noticed that the negative values of the third moment indicate skewness of the distribution to the right. This behavior is quite expected for PDFs defined at centerline near the source where the initial concentration $\left(C_{0}\right)$ still dominates due to weak influence of molecular diffusion and, as diffusion takes over, the distribution is gradually shifted to the left (Figs. 5.10 to 5.12). Lower values of the fourth moment indicate steepness of the PDF and, as expected, it is again found at the centerline near the source, due to two state distribution being focused in $C_{0}$, while the other minimums correspond to maximum variance in the near-field profiles. This is explained by the dissipation of concentration values (due to the source geometry) to mostly 0 and $C_{0}$, which explains the high variance and local minimums of the fourth moment since those two peaks are steep. For higher moments including the fifth one and above, no direct meaning to PDF geometry is available. They do, however, participate in the definitions of the distribution tails and bring valuable information when PDF inversion is performed using the MaxEnt formulation (Chapter 2). They may also cause an issue in numerical stability when using maximum entropy algorithms, since they add additional dimensions when calculating Lagrangian multipliers (2.3.2). Therefore, usually only the first four are used, and when applying moment matching to a 2-parameter distribution (e.g. Beta distribution), it is acceptable to use only the first two.

Furthermore, the impact of molecular diffusion and the velocity attenuation coefficient on concentration moments was analyzed. 


\section{The influence of molecular diffusion on concentration central moments}

Figs. 5.3 to 5.5 show the impact of molecular diffusion on the second, third, and fourth concentration central moments, respectively. Molecular diffusion acts as a slow process as compared to turbulent advection, once again supporting the assumption that these two competing processes act on different timescales. Thus, by going further from the river mouth, the impact of molecular diffusion becomes more important, leading to a significant reduction in the peak of concentration moments.
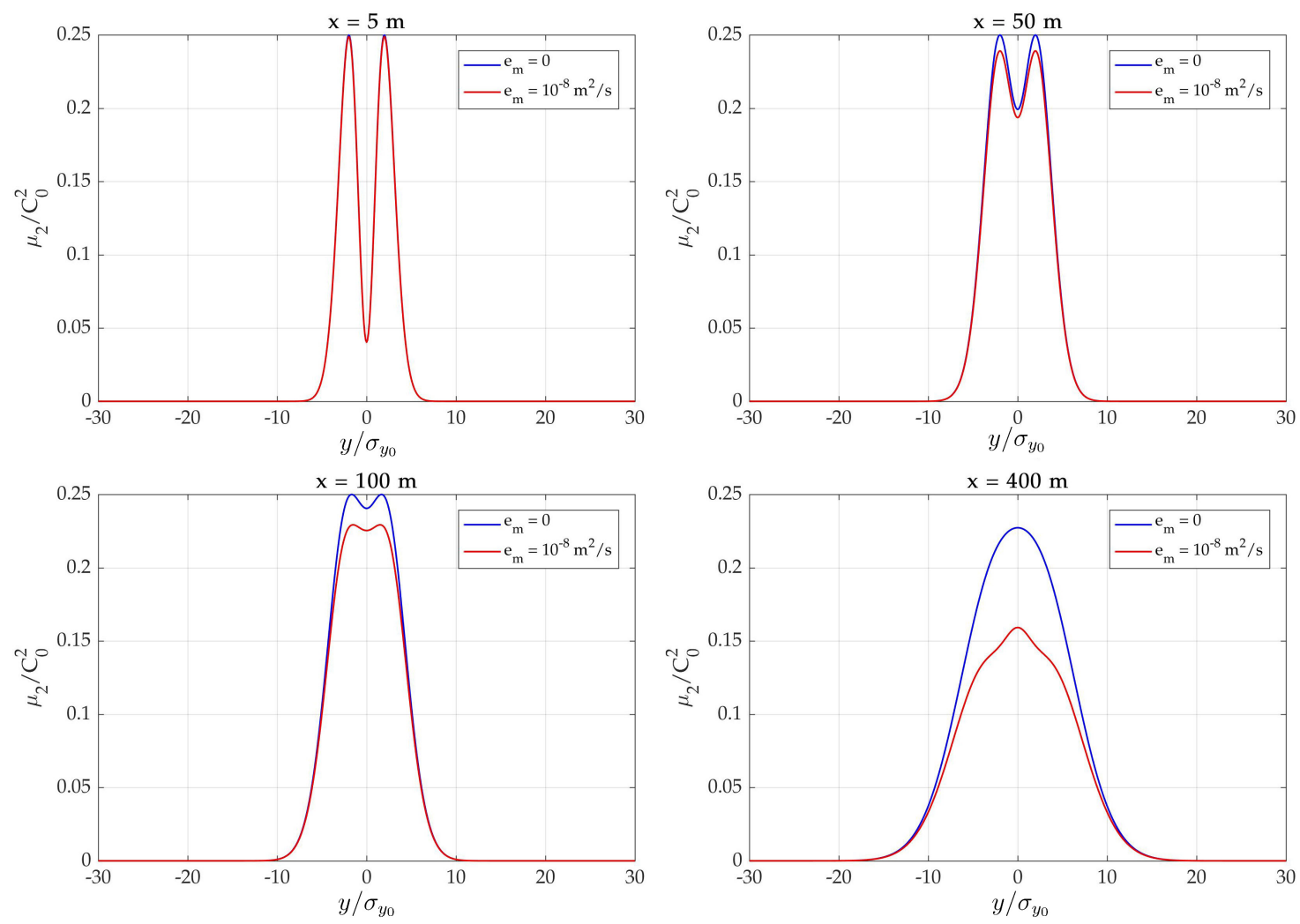

FIGURE 5.3: The influence of molecular diffusion on the second central moment $\mu_{2}$, at different distances from the source.

In the case of concentration variance, the shape changes from a bimodal to a unimodal one at large enough distances from the source (Fig. 5.3), as already stated above and previously observed by [4, 127, 140, 5, 137]. 

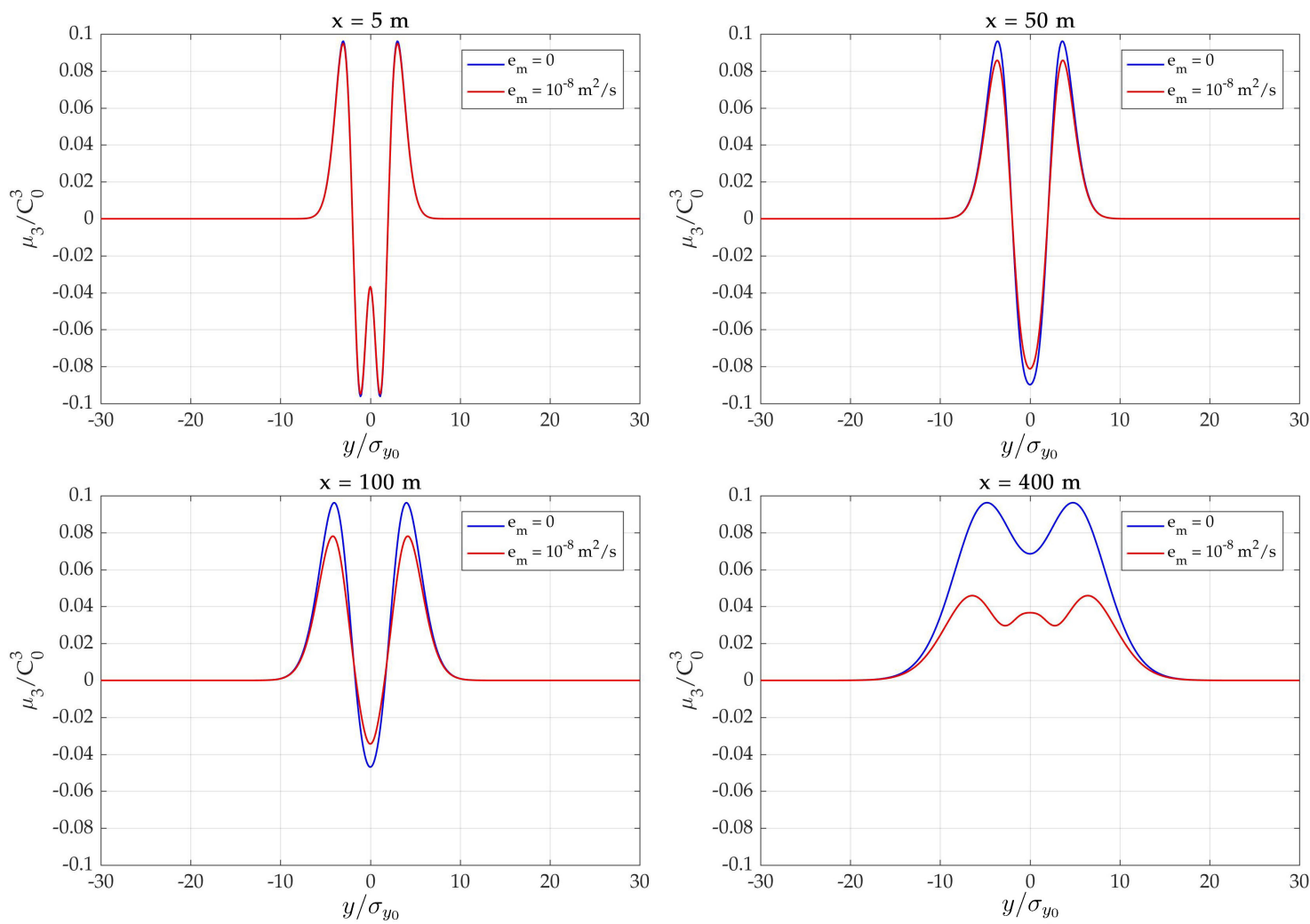

FIGURE 5.4: The influence of molecular diffusion on the third central moment $\mu_{3}$, at different distances from the source.
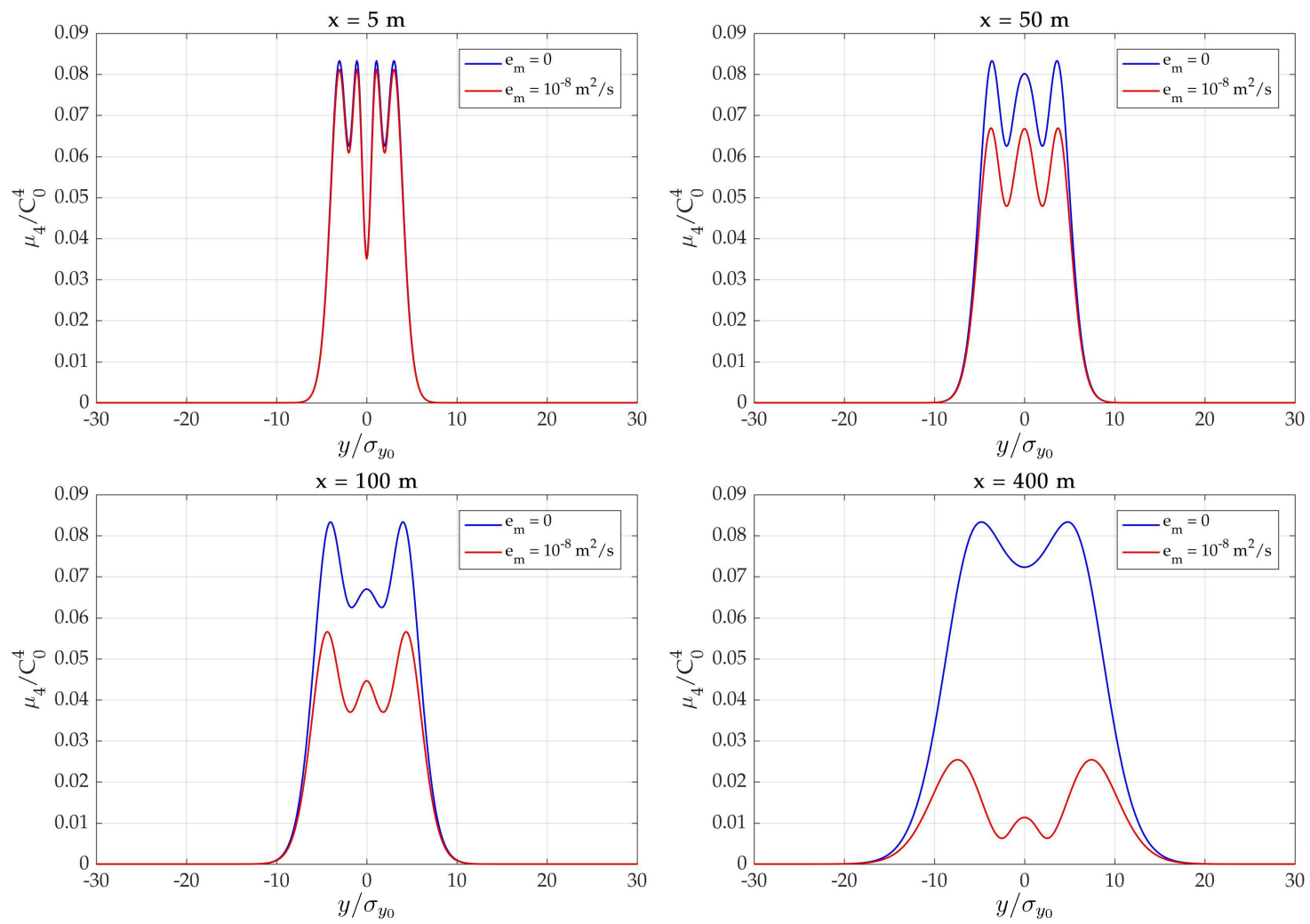

FIGURE 5.5: The influence of molecular diffusion on the fourth central moment $\mu_{4}$, at different distances from the source. 


\section{The influence of mean velocity attenuation on concentration central moments}

Additionally, the influence of the velocity attenuation coefficient is investigated. For very small absolute values of the attenuation coefficient (e.g. $\nu<0.002 \mathrm{~m}^{-1}$ ), velocity may be considered to be constant, yielding minor effects on concentration moments, which is easily observed in the figures below, and emphasized in the near source profiles.
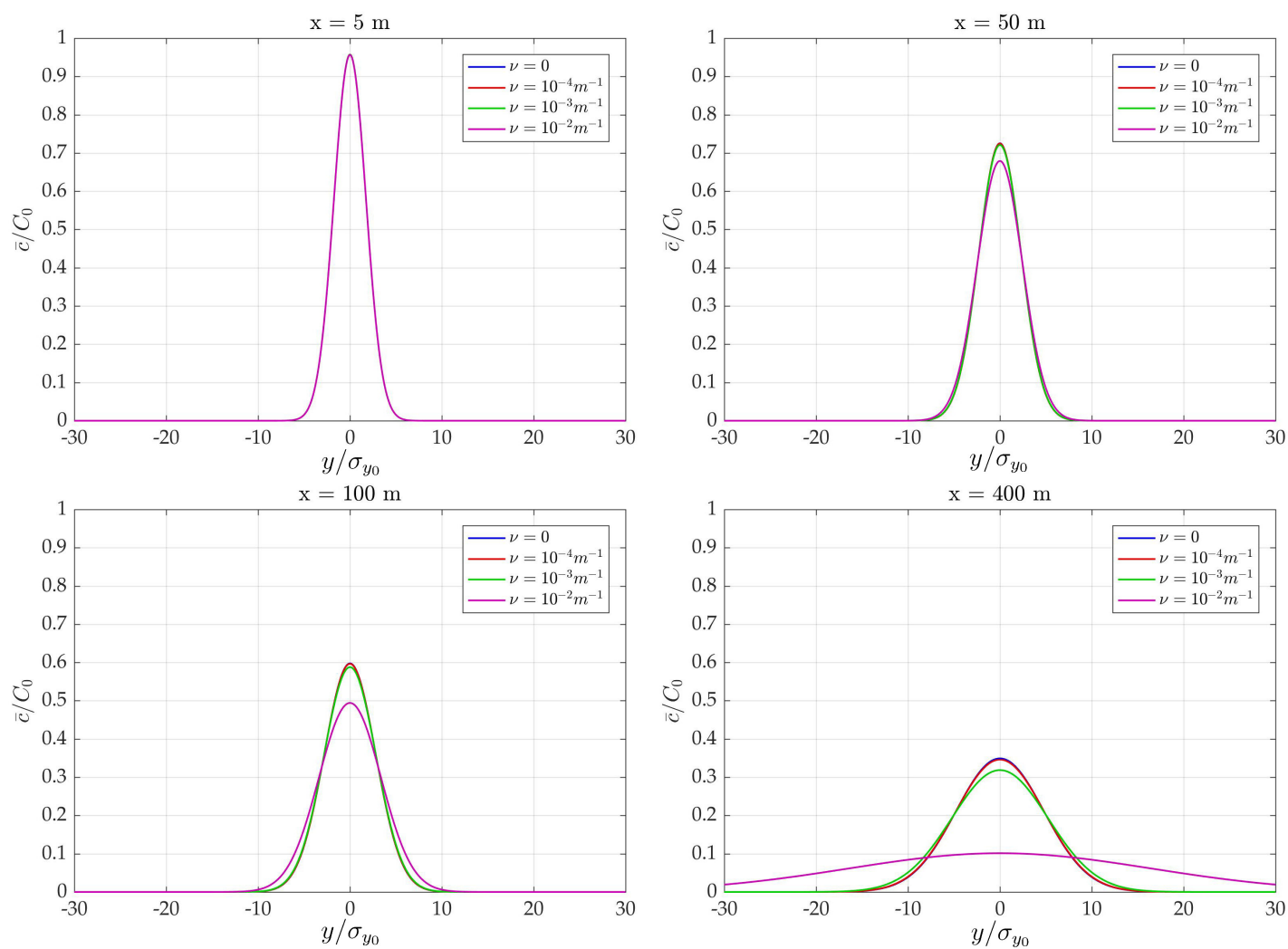

FIGURE 5.6: The influence of mean velocity attenuation on mean concentration $\bar{c}$, at different distances from the source.

Fig. 5.6 illustrates the effect of velocity attenuation on mean concentration. As expected, in the first two panels $(x=5 \mathrm{~m}$ and $x=50 \mathrm{~m})$, the differences are negligible. From $400 \mathrm{~m}$ onward, considering a strong attenuation coefficient $\nu=0.01$ $\mathrm{m}^{-1}$, a noticeable reduction in the mean concentration can be observed, since the effect of $\nu$ is increasing with downstream distance. The river discharge creates a plume of buoyant freshwater which is slowly dispersed due to the mixing processes with ambient seawater along its path. Thus, this proposed attenuation coefficient may represent a convenient parameter to cover the downstream effects on the plume. 
The second, third, and fourth concentration moments for three different velocity attenuation coefficients are presented in Figs. 5.7 to 5.9, respectively. The key finding is that the value of the attenuation coefficient resulting from field velocity measurements $\left(\nu=0.002 \mathrm{~m}^{-1}\right)$ does not have a significant impact on concentration moments $(n=2,3,4)$ up to $400 \mathrm{~m}$ from the source.
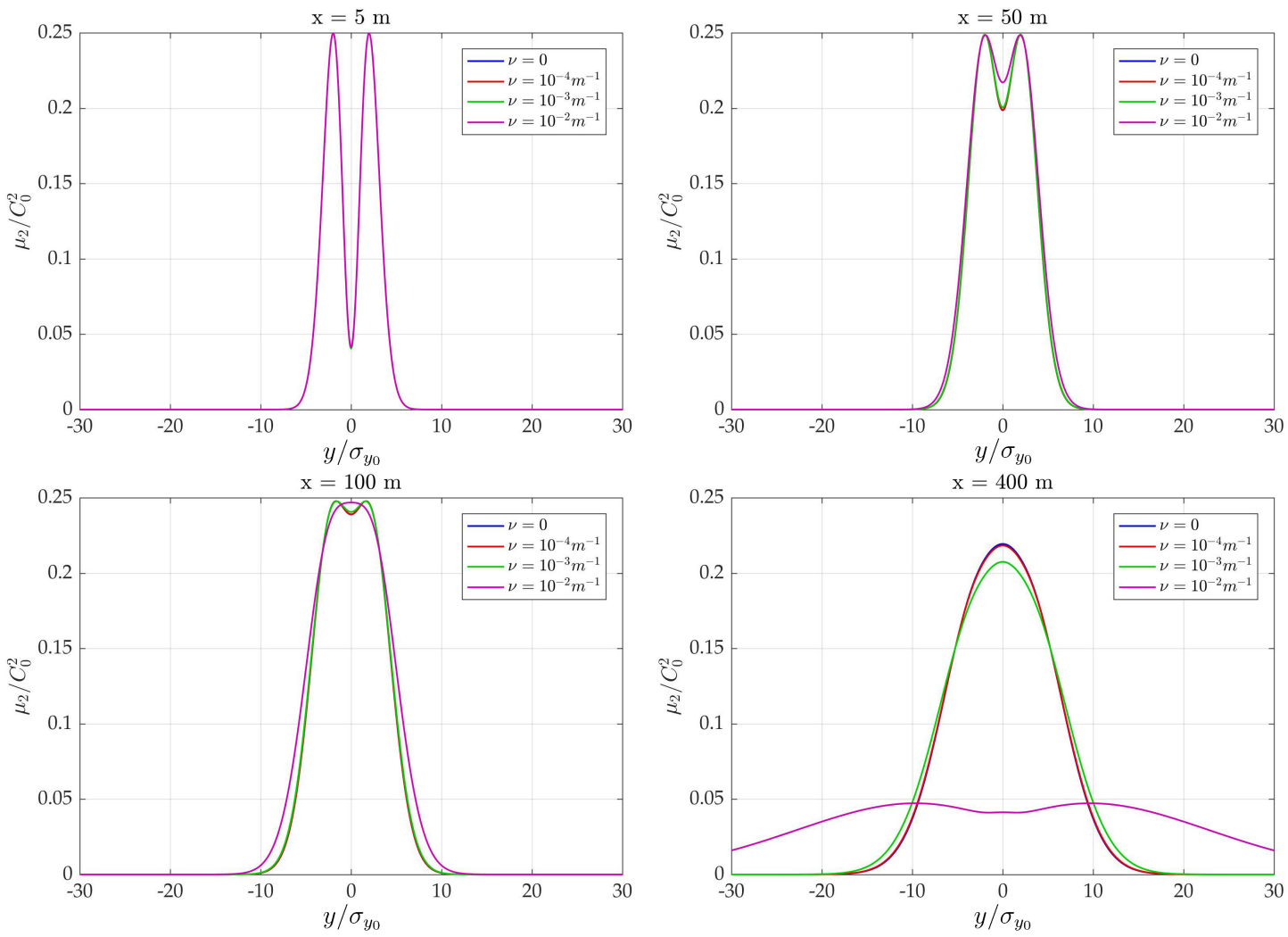

FIGURE 5.7: The influence of mean velocity attenuation on the second central moment $\mu_{2}$, at different distances from the source.

Higher values of the attenuation coefficient (e.g. $\nu=0.01 \mathrm{~m}^{-1}$ ), which could indicate conditions of a strong sea tide and wind-induced currents, show a significant reduction in concentration moments for $x=400 \mathrm{~m}$, and the fourth concentration moment even exhibits non-physical negative values around the centerline. Similar behavior of the fourth concentration moment was also observed in the atmospheric diffusion problem by Sullivan [140], as a result of the limitation embodied by the assumption (Eq. (2.7)). 

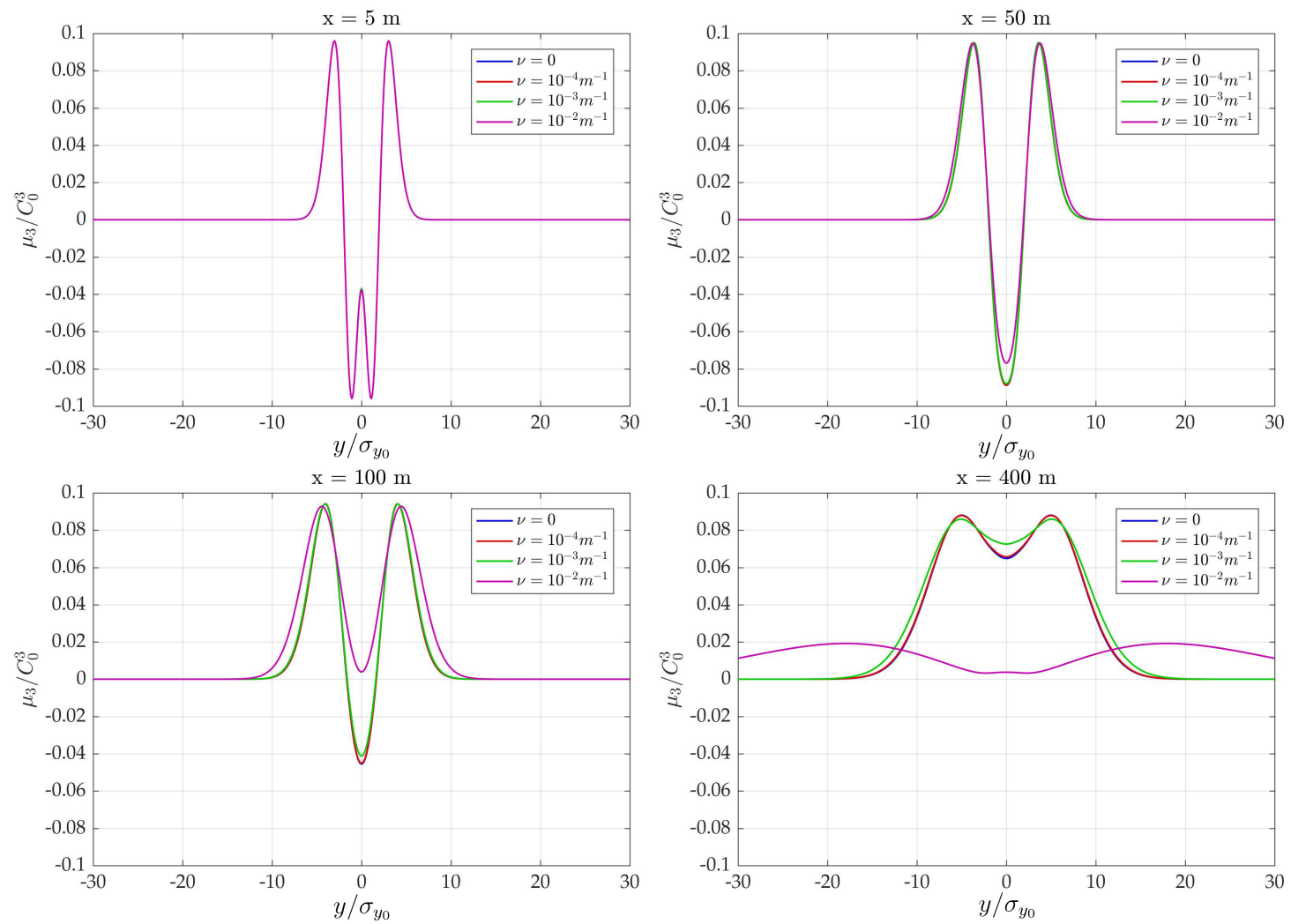

FIGURE 5.8: The influence of mean velocity attenuation on the third central moment $\mu_{3}$, at different distances from the source.
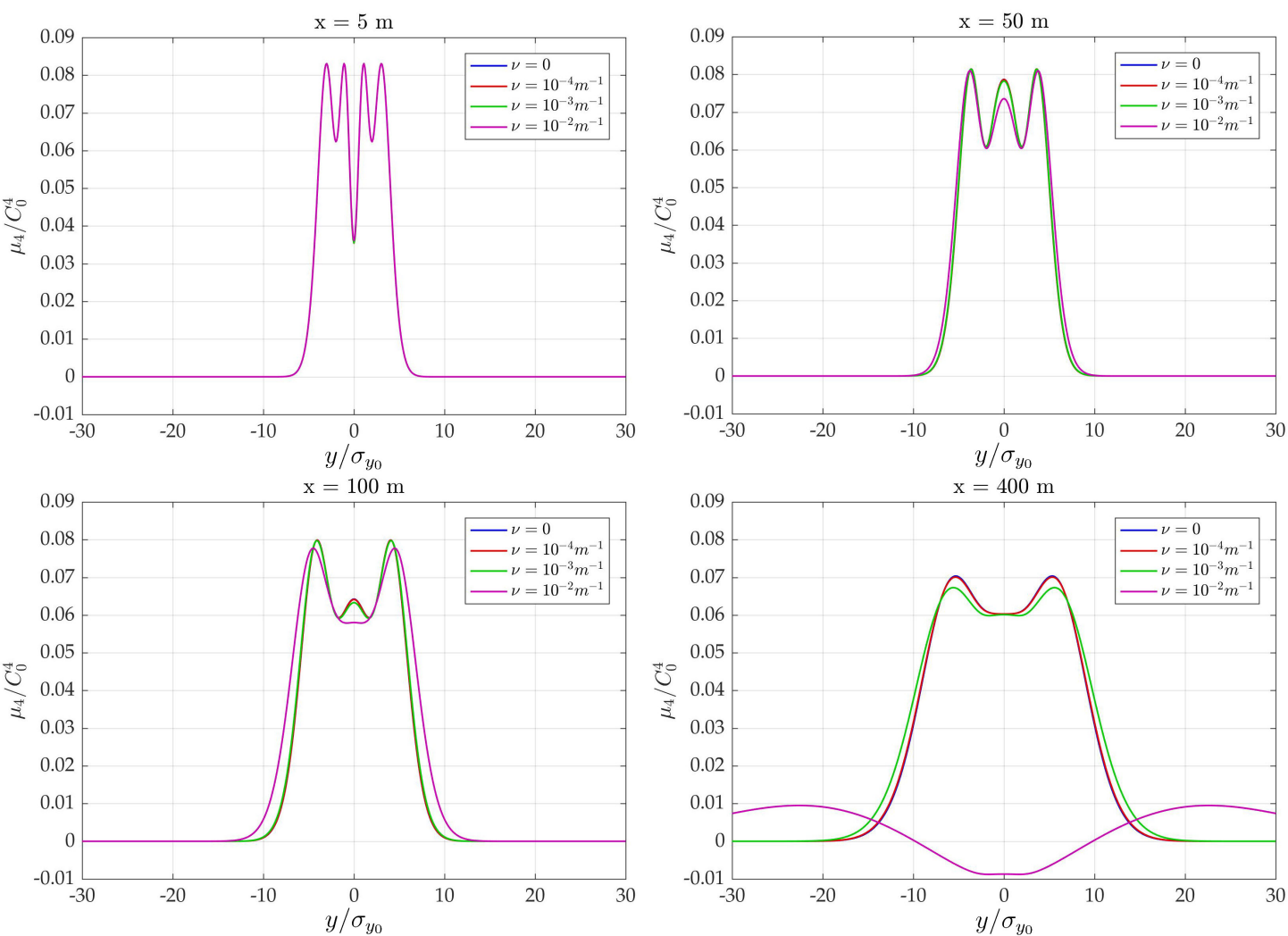

FIGURE 5.9: The influence of mean velocity attenuation on the fourth central moment $\mu_{4}$, at different distances from the source. 
A strong attenuation coefficient, in fact, reduces turbulent advection and speeds up the relative impact of molecular diffusion. The strength of the attenuation coefficient may thus control the acceptable size of the near-field zone where approximation (Eq. (2.7)) holds, as further discussed in Section 5.4.4 and shown in Fig. 5.45.

\section{PDFs and CDFs obtained from concentration moments}

Following Section 2.2 and Section 2.3, PDFs and CDFs are evaluated by inversion of concentration moments. In Figs. 5.10 to 5.12, PDFs (left panel) and CDFs (right panel) are presented for five different distances from the source $(x=$ $5,50,100,200,400 \mathrm{~m}$ ), with an emphasis on different cases of mean velocity attenuation.
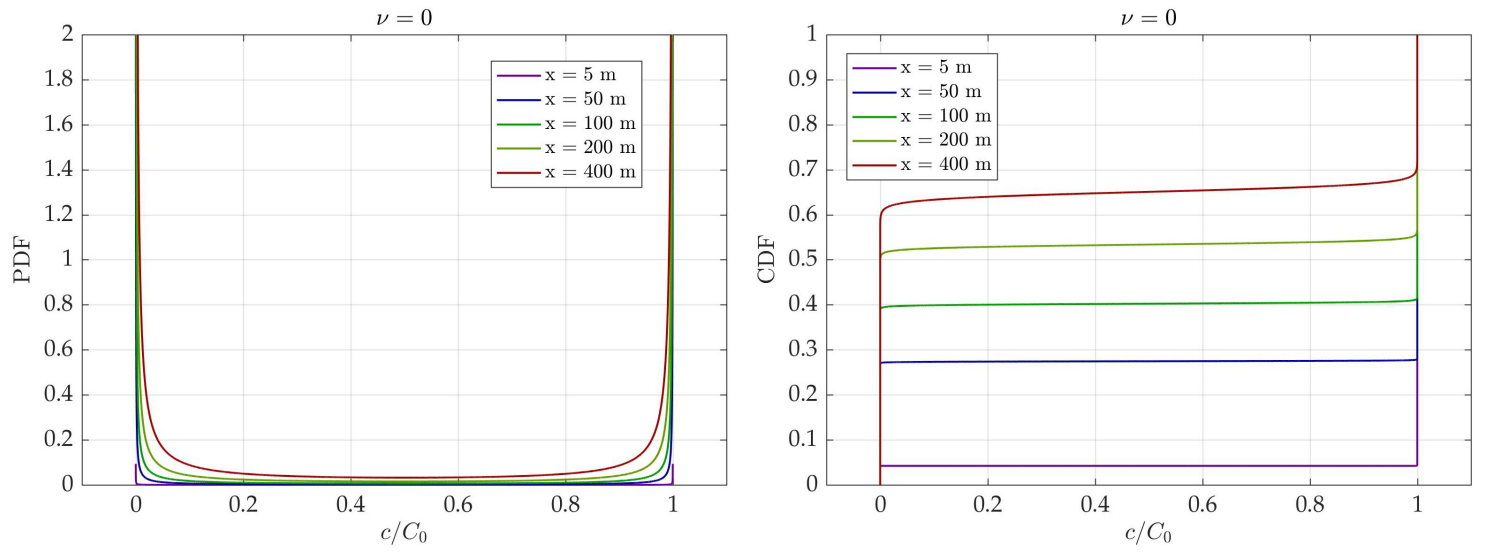

FIGURE 5.10: The concentration Beta distribution PDFs (left) and CDFs (right) for the velocity attenuation coefficient $\nu=0$, and for five different distances $(x=5,50,100,200,400 \mathrm{~m})$ from the source at centerline.

The structure of concentration moments with and without molecular diffusion differs slightly in the near-field zone before molecular diffusion takes over as a dominating process. In the case of pure advection of a constant concentration source $C_{0}$, the probability density function is a two-state distribution described by the double Dirac function at 0 and $C_{0}$. However, as the distance from the source increases, molecular diffusion gains momentum, which is reflected by lowering impulses and increasing the surface of the concentration PDF between 0 and $C_{0}$. That surface predominately represents intermittency, since intermittency is the 
probability that a fluid at certain $(\mathbf{x}, t)$ is coming from the source. This definition of intermittency was introduced by [28], and it conveys valuable information about the effect of the velocity field [105].
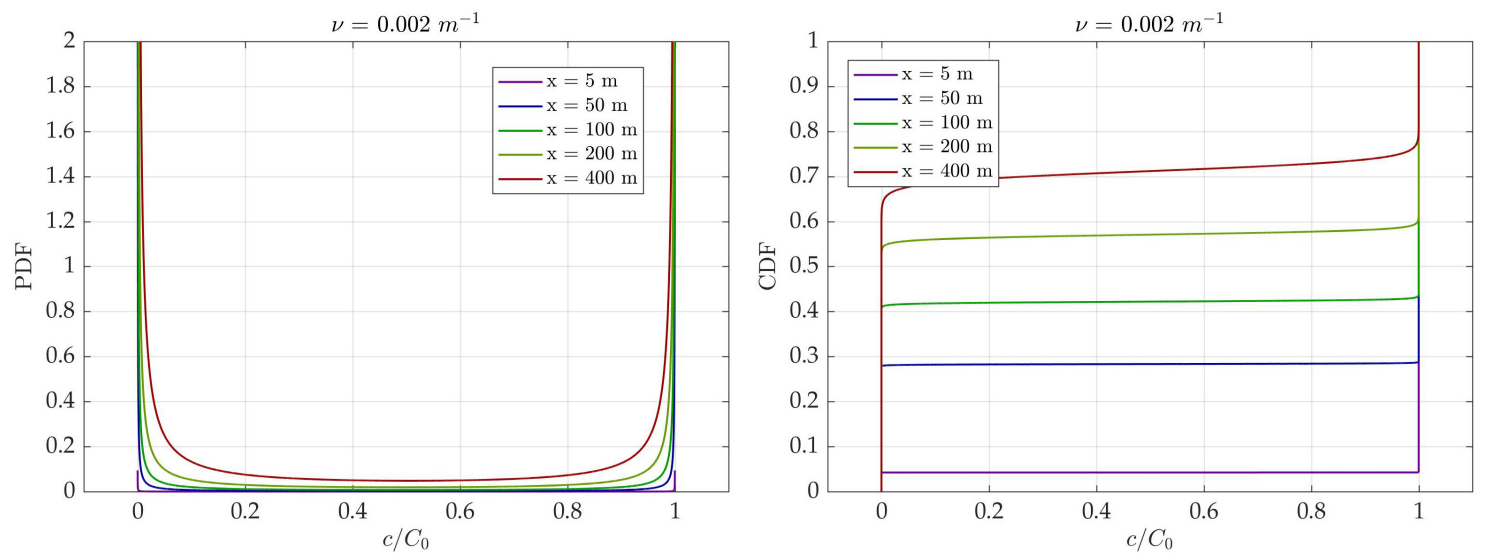

FIGURE 5.11: The concentration Beta distribution PDFs (left) and CDFs (right) for the velocity attenuation coefficient $\nu=0.002 \mathrm{~m}^{-1}$, and for five different distances $(x=5,50,100,200,400 \mathrm{~m})$ from the source at centerline.
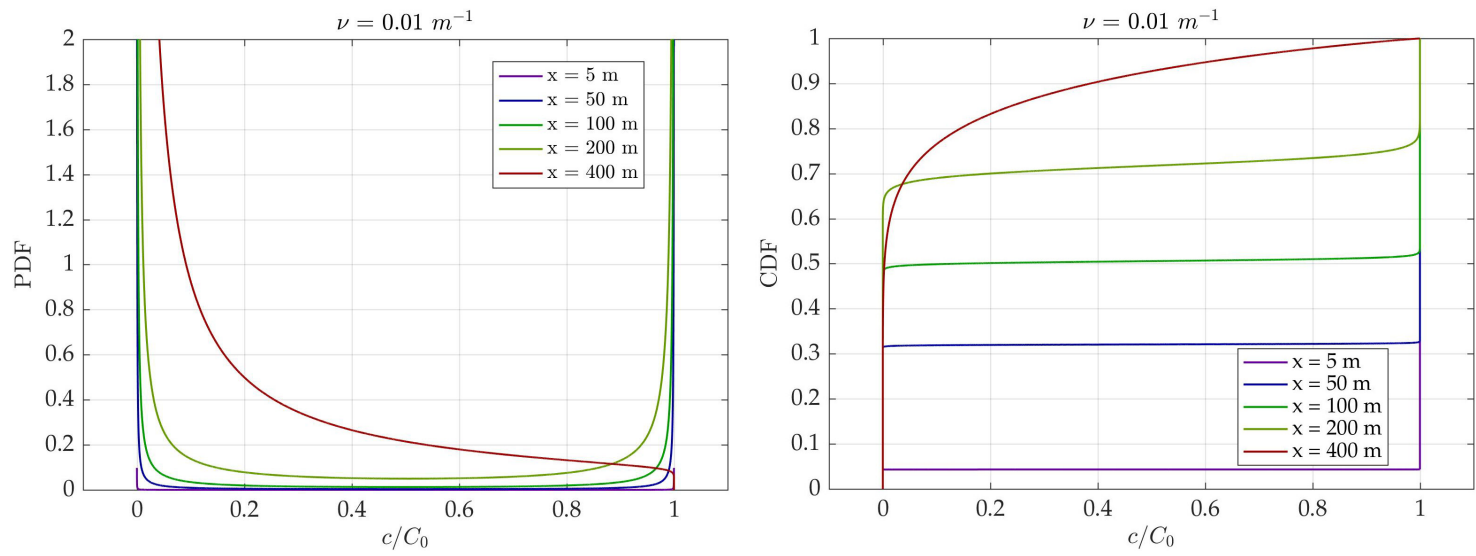

FIGURE 5.12: The concentration Beta distribution PDFs (left) and CDFs (right) for the velocity attenuation coefficient $\nu=0.01 \mathrm{~m}^{-1}$, and for five different distances $(x=5,50,100,200,400 \mathrm{~m})$ from the source at centerline.

The behavior of intermittency, as the probability of a fluid element coming from the source, can be seen in all three Figs. 5.10 to 5.12 (left panel), where the initial PDFs at a 5-meter distance from the source are very similar to the double Dirac functions. However, moving along the centerline, PDFs indicate intermittency growth and widening of the base as the impact of molecular diffusion is 
slowly increasing along the downstream direction. The corresponding concentration cumulative density functions (CDFs) are presented on the right-hand panels of the aforementioned figures. In CDFs plot, it can easily be noticed that the probability of having zero concentration is practically non-existent within a 5-meter distance from the source. Three different attenuation coefficients are investigated and their influence presented in these figures. It is interesting to observe that the attenuation coefficient of the mean velocity makes the concentration PDF positively skewed, which is more pronounced as one moves along the downstream direction. This is even more emphasized with larger velocity attenuation such as $\nu=0.01 \mathrm{~m}^{-1}$ (Fig. 5.12). This behavior, once again, indicates that the attenuation of mean velocity increases the lateral spreading of the concentration due to a quicker influence of the mixing processes on the river plume. Apparently, the mean velocity attenuation coefficient is an important parameter for the problem of concentration statistics in an estuary and indicates the necessity of velocity field measurements.

\subsubsection{Integrated concentration statistics}

Chapter 3 described the methodology for the application of the EMF concept in an estuary. In this section, different variations of results are presented in order to demonstrate the potential of the EMF as a concentration dilution measure in the particular estuary of the Žnovnica River. Therefore, parameters used in calculations are similar to the setup used for the demonstration of point statistics; however, the mouth width is set at $b=14 \mathrm{~m}$, the initial velocity is $0.4 \mathrm{~m} / \mathrm{s}$, and mean velocity attenuation is tested only for the measured one of $0.002 \mathrm{~m}^{-1}$ (Fig. 5.20) in comparison to constant mean velocity. Moreover, different intensities of the initial mass loading in the form of a steady flux $(\dot{m})$ are investigated and compared. The results are presented for the method of obtaining the EMF directly by spatial integration of Beta PDFs (Section 3.2) and for the EMFs obtained by inversion of its moments.

\section{Direct integration of PDFs}

As represented by Fig. 3.3, a direct integration over the perpendicular downstream slice with the width $\Delta x=1 \mathrm{~m}$ is performed by Eq. (3.11) for the case of constant mean velocity and the attenuated one $\left(\nu=0.002 \mathrm{~m}^{-1}\right)$. The EMFs are obtained for an estimated worst-case scenario of mass loading with a steady flux 
of $\dot{m}=50 \mathrm{~g} / \mathrm{s}$ (Fig. 5.13) at different distances from the source $x=5,50,100,200$, $400,600 \mathrm{~m}$.
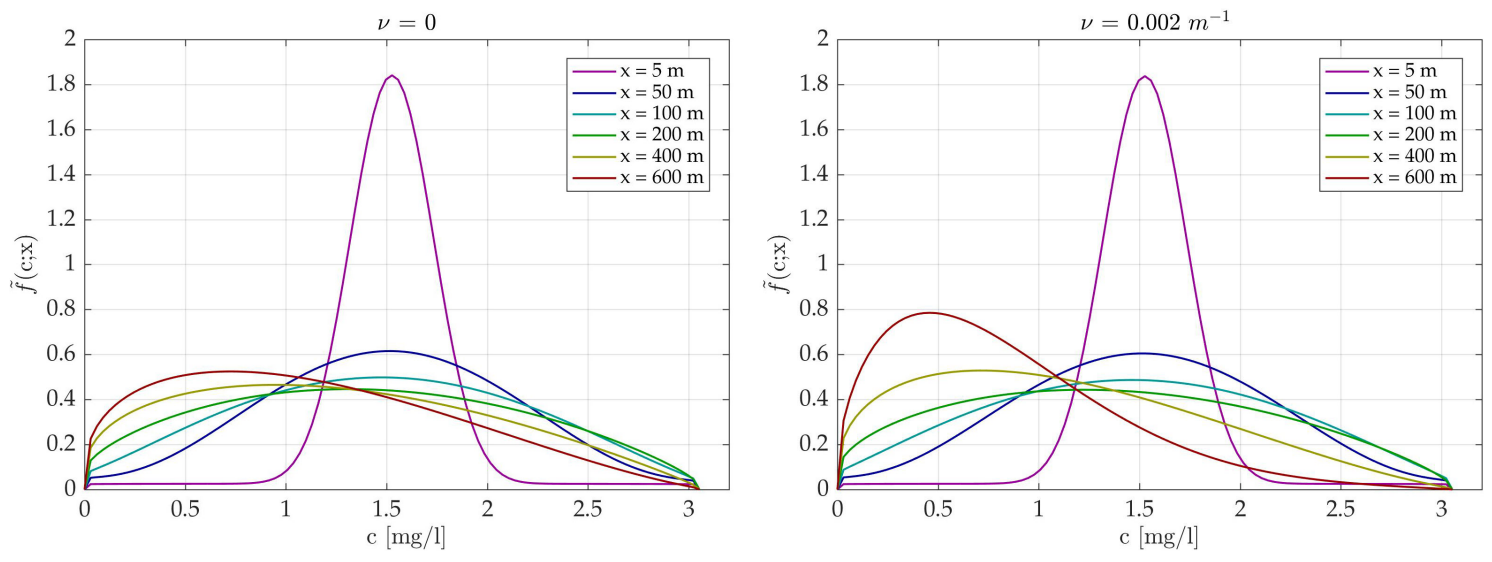

FIGURE 5.13: EMFs obtained at different distances from the source, by direct spatial integration of point Beta distribution PDFs, for constant mean velocity (left) and for a decreasing mean velocity with the attenuation factor of $\nu=0.002 \mathrm{~m}^{-1}$ (right).

Again, the effect of mean velocity attenuation is more pronounced at larger distances (e.g. for $x=400$ or $600 \mathrm{~m}$ ), where more expected mass exhibits lower values of concentration in comparison to constant mean velocity. Molecular diffusion takes momentum and reduces the concentration more quickly since the advection process is decreased by the attenuated velocity. The effect of the different mass loading in the term of a steady flux $(\dot{m})$ is investigated and presented in Fig. 5.14.
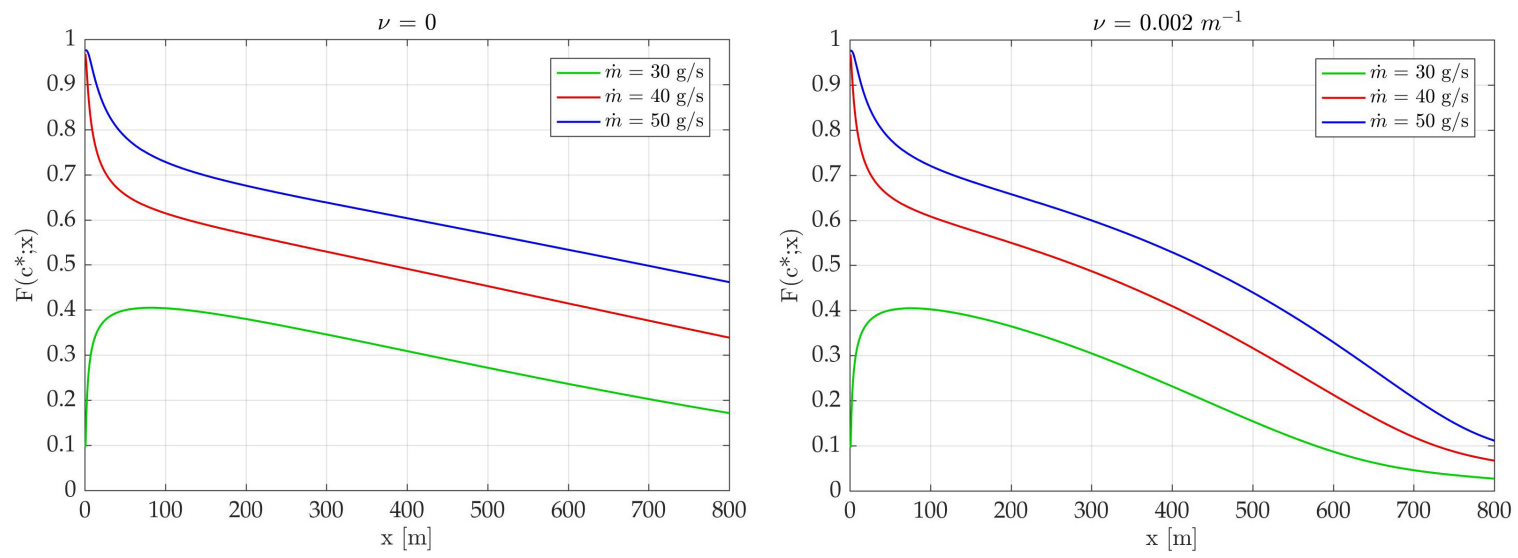

FIGURE 5.14: Contaminant mass fraction exhibiting concentration levels above the limit value $\left(c^{*}=1 \mathrm{mg} / \mathrm{l}\right)$, for constant mean velocity (left) and for a decreasing mean velocity with the attenuation factor of $\nu=0.002 \mathrm{~m}^{-1}$ (right), with different loadings. 
An arbitrary limit value of the concentration is chosen, $c^{*}=1 \mathrm{mg} / \mathrm{l}$, which corresponds to the limit value of total nitrogen for the best quality water as defined by Croatian regulations and based on the WFD. The evolution of mass fraction, along the downstream direction, exhibiting concentration above $c^{*}$ for different contaminant steady fluxes delivers a potential estimate of concentration dilution. For instance, if $20 \%$ of steady mass loading $(L)$ with concentration values above $c^{*}$ may be considered as acceptable, then the distances at which they are achieved may indicate the area needed for the dilution process to be mostly completed. In case of attenuated mean velocity (see Fig. 5.14, right panel), such distances are:

- $x=450 \mathrm{~m}$ for $\dot{m}=30 \mathrm{~g} / \mathrm{s}$

- $x=600 \mathrm{~m}$ for $\dot{m}=40 \mathrm{~g} / \mathrm{s}$

- $x=700 \mathrm{~m}$ for $\dot{m}=50 \mathrm{~g} / \mathrm{s}$

However, in the case of a constant mean velocity (the left panel of Fig. 5.14), where molecular diffusion acts later, those distances are all greater than $700 \mathrm{~m}$. These effects are going to be further discussed and compared to EMFs obtained by inversion from its moments in Section 5.4.

\section{Spatially integrated concentration moments and their application for obtain- ing the EMF}

Different cases of mass loading $(\dot{m})$ and the effect of velocity attenuation (measured value of $\nu=0.002 \mathrm{~m}^{-1}$ ) are investigated when obtaining spatially integrated moments $K_{n}$ and the corresponding EMF moments $\tilde{m}_{n}$. Since these moments produce very similar results when obtained by integration of point PDF absolute moments $\left(m_{n}\right)$ or by using a recursive solution (Eq. (3.22)) as explained in Section 3.3, the results presented here correspond to both Section 3.2 and Section 3.3. $K_{0}$ and $K_{1}$ are equal to the all space volume (the perpendicular profile with the area defined $2 \kappa \sigma_{y}(x) \Delta x$ ) and a steady mass loading $(L)$ as shown in Fig. 3.5. Here, $K_{2}, K_{3}$, and $K_{4}$ are shown with respect to different loading and mean velocity attenuation (Fig. 5.15). All three moments show an exponential decrease along the downstream direction as previously observed by [120]. The EMF moments $\left(\tilde{m}_{n}\right)$ are directly related to one order higher spatially integrated moments (Eq. (3.7)), which is observed in their similar shape (Fig. 5.16).

As opposed to examples shown in Chapter 3, both $K_{n}$ and $\tilde{m}_{n}$ are shown in a non-normalized form to properly demonstrate the difference caused by the mass loading input. Since an actual steady mass flux $[\mathrm{g} / \mathrm{s}]$ or, in general, dimensional 

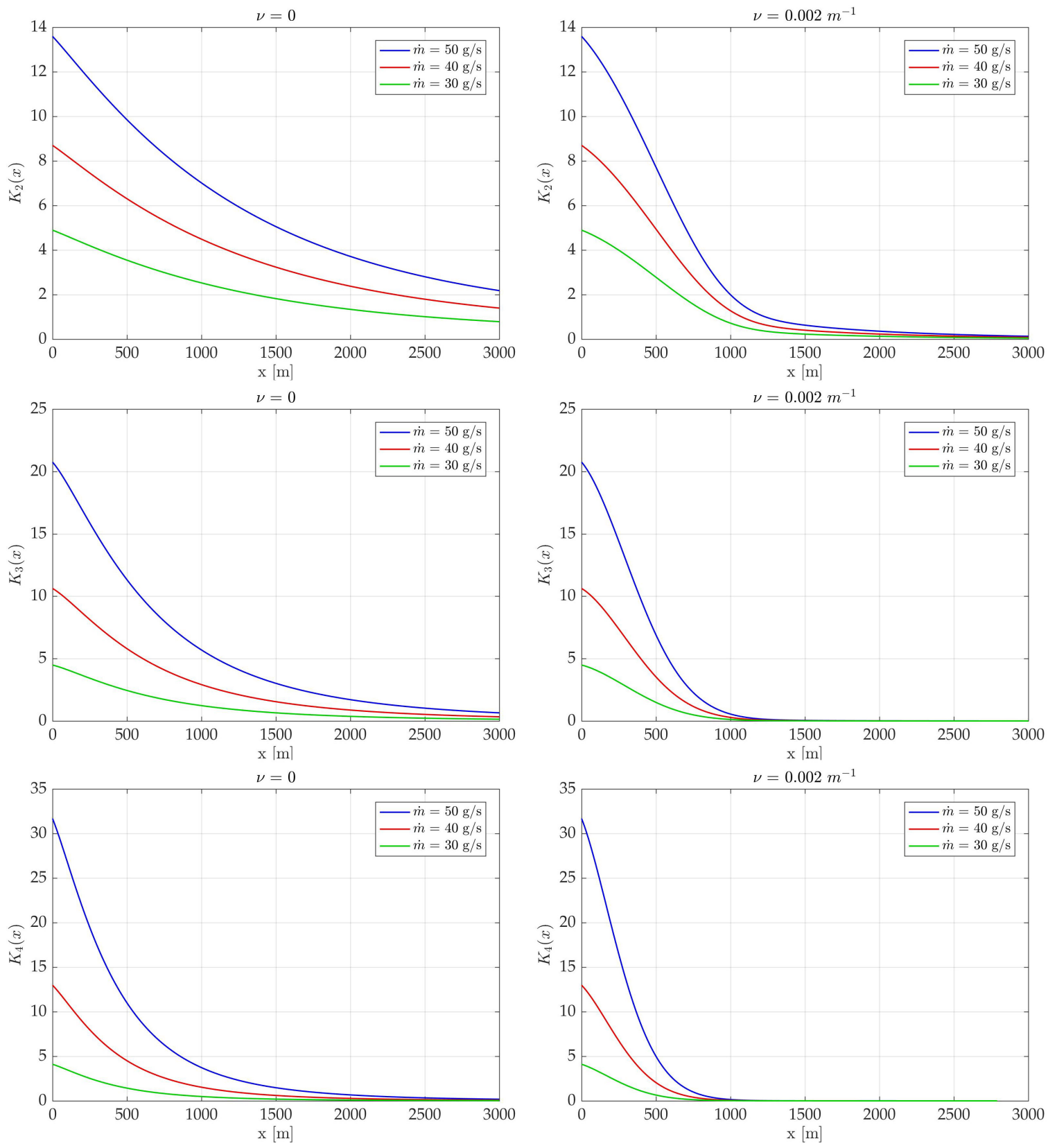

FIGURE 5.15: Spatially integrated moments - $K_{2}\left[\mathrm{mg}^{2} / \mathrm{l}\right], K_{3}$ $\left[\mathrm{mg}^{3} / \mathrm{l}^{2}\right]$, and $K_{4}\left[\mathrm{mg}^{4} / 1^{3}\right]$, for constant mean velocity (left panel) and for a decreasing mean velocity with the attenuation factor of $\nu=0.002 \mathrm{~m}^{-1}$ (right panel), with different loadings.

analysis [M/T] was used in these examples, the corresponding measure units for spatially integrated moments of the $\mathrm{n}^{\text {th }}$ order are $\mathrm{mg}^{\mathrm{n}} / \mathrm{l}^{\mathrm{n}-1}\left(\mathrm{M}^{\mathrm{n}} / \mathrm{L}^{3(\mathrm{n}-1)}\right)$. Hence, the EMF moments of the $\mathrm{n}^{\text {th }}$ order have $\mathrm{mg}^{\mathrm{n}} / \mathrm{l}^{\mathrm{n}}\left(\mathrm{M}^{\mathrm{n}} / \mathrm{L}^{3 \mathrm{n}}\right)$ as measure units, according to Eq. (3.7), which indicates direct similarities of point PDF moments to EMF moments. Therefore, the first EMF moment $\left(\tilde{m}_{1},[\mathrm{mg} / \mathrm{l}]\right.$ or $\left.\left[\mathrm{M} / \mathrm{L}^{3}\right]\right)$ corresponds to mean concentration, representing a spatial mean concentration over all space, or 

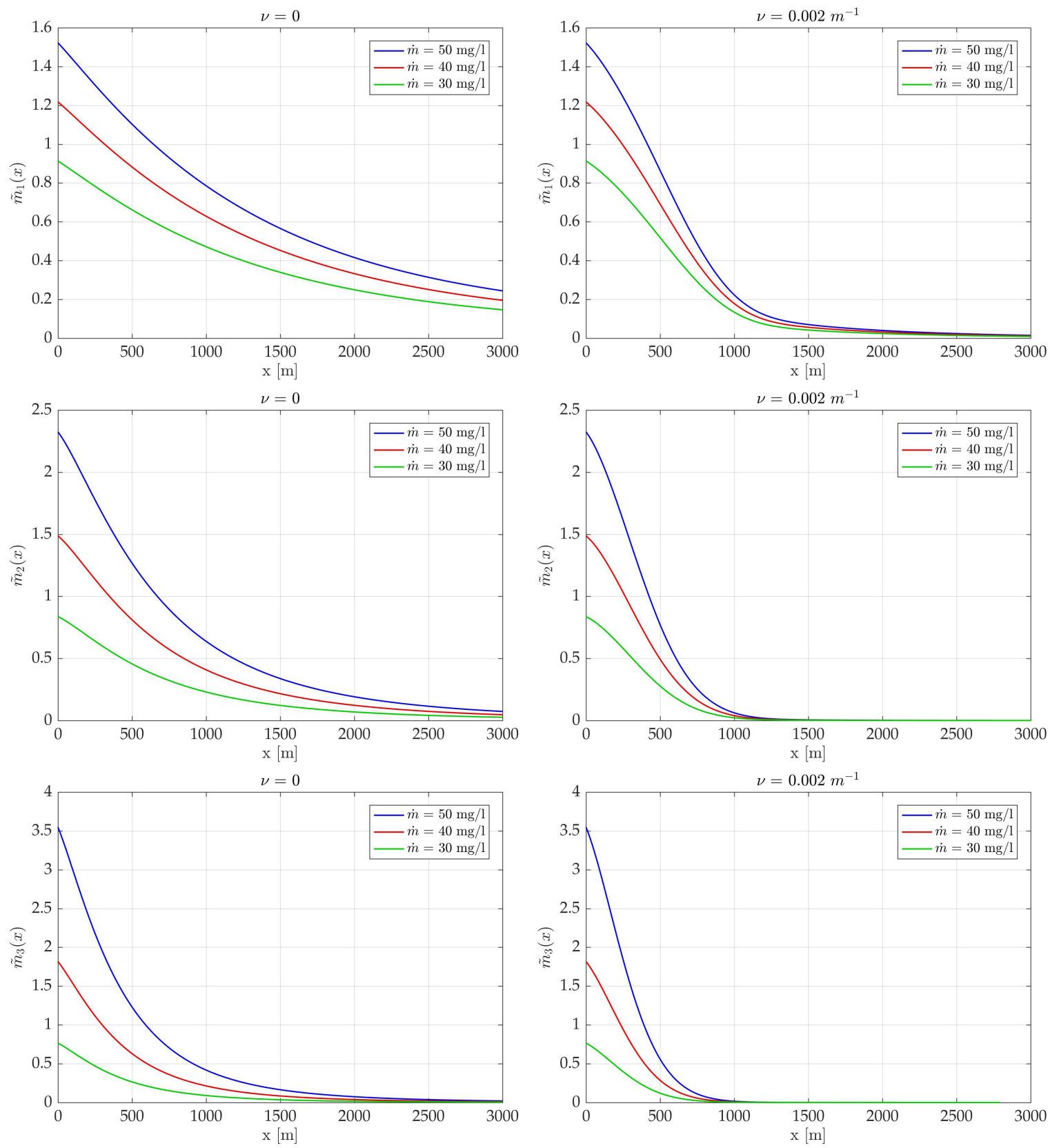

FIGURE 5.16: EMF moments $\tilde{m}_{1}[\mathrm{mg} / \mathrm{l}], \tilde{m}_{2}\left[\mathrm{mg}^{2} / \mathrm{l}^{2}\right]$, and $\tilde{m}_{3}$ $\left[\mathrm{mg}^{3} / \mathrm{l}^{3}\right]$, for constant mean velocity (left panel) and for a decreasing mean velocity with the attenuation factor of $\nu=0.002 \mathrm{~m}^{-1}$ (right panel), with different loadings.

a specific surface of perpendicular profile in these examples. Similarly, the second $\left(\mathrm{M}^{2} / \mathrm{L}^{6}\right)$ and the third $\left(\mathrm{M}^{3} / \mathrm{L}^{9}\right)$ EMF moment would indicate spatial variation of concentration and skewness of the corresponding EMF function, respectively.

This inference makes EMF moments very useful when describing concentration field reduction along the downstream direction. As expected, higher values of a steady mass flux cause initial disparities between each of the moments, but 

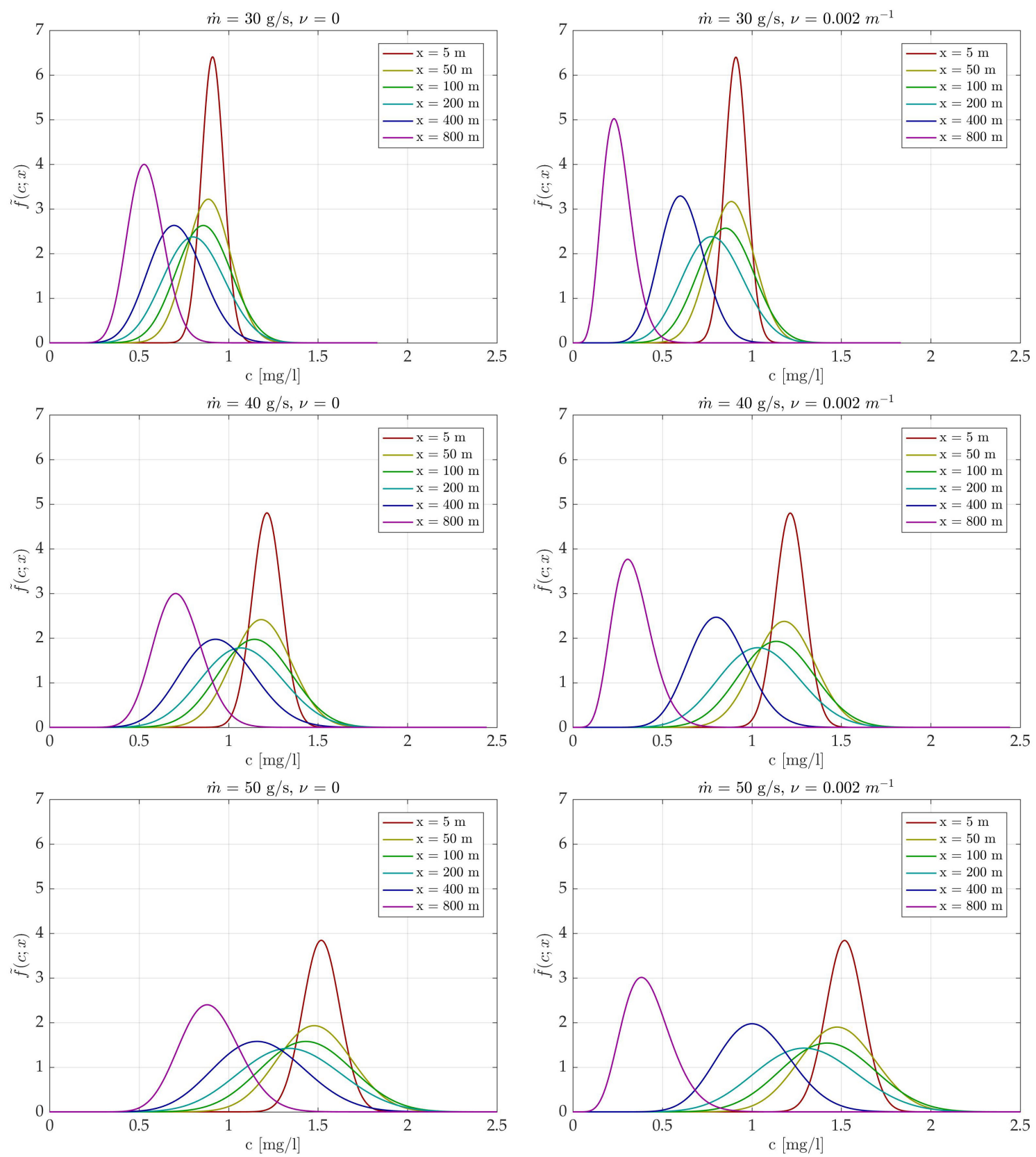

FIGURE 5.17: EMFs obtained by inversion of their moments at different distances from the source, for constant mean velocity (left panel) and for a decreasing mean velocity with the attenuation factor of $\nu=0.002 \mathrm{~m}^{-1}$ (right panel), with different loadings.

those tend to drastically decrease further from source.

At the larger distances, the influence of the source is diminished and molecular diffusion takes over, causing approximate convergence of moment values. This behavior is pronounced after $3000 \mathrm{~m}$ for all moments $\left(K_{n}\right.$ and $\left.\tilde{m}_{n}\right)$ in case of constant mean velocity (both Fig. 5.15 and Fig. 5.16, left panel). Mean velocity attenuation, once again, delivers the results, which indicate earlier advancement 
of molecular diffusion (both Fig. 5.15 and Fig. 5.16, right panel). In the attenuated case, convergence and total reduction of all moments is achieved already at $1000 \mathrm{~m}$. Although spatially integrated moments and the corresponding EMF moments are quite interesting to analyze, their inversion for obtaining EMF functions enables even better understanding of the dilution process characteristics (Fig. 5.17). Following the Beta distribution EMFs along the downstream direction, the changes in mass fraction exhibiting ever-decreasing levels of concentration can easily be observed. It is particularly interesting to see the impact of different steady mass flux inputs and the impact of mean velocity attenuation (the left panel as opposed to the right one in Fig. 5.17). Unsurprisingly, the higher value of steady mass flux results in longer presence of higher values of concentration.
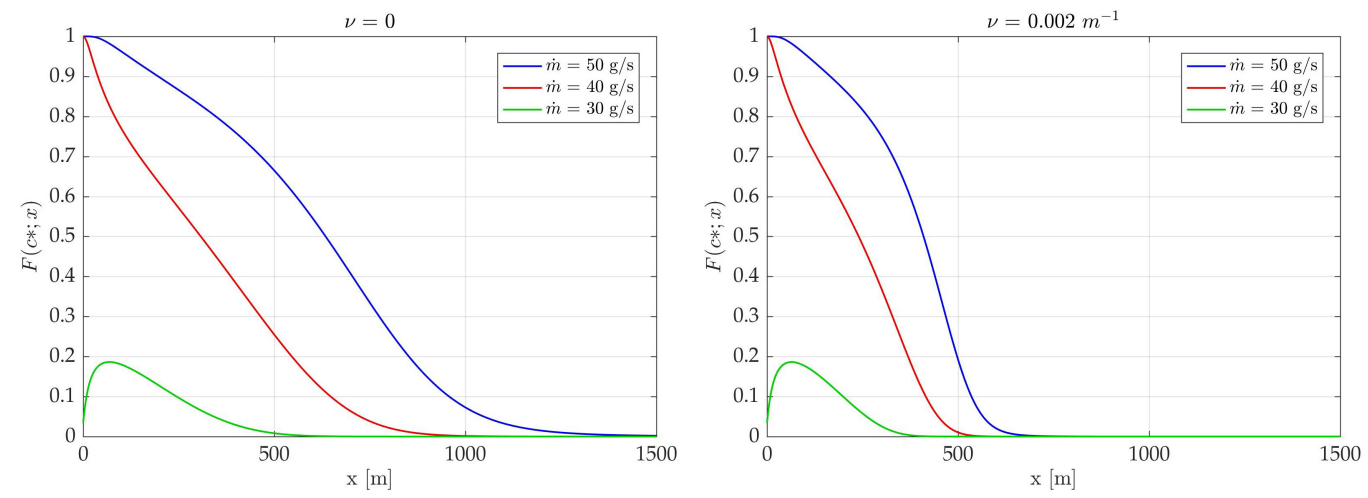

FIGURE 5.18: Contaminant mass fraction exhibiting concentration levels above the limit value $\left(c^{*}=1 \mathrm{mg} / \mathrm{l}\right)$, obtained by spatially integrated moments, for constant mean velocity (left) and for a decreasing mean velocity with the attenuation factor of $\nu=0.002 \mathrm{~m}^{-1}$

(right), with different loadings.

Mean velocity attenuation, as expected, reduces the dilution domain due to a relatively accelerated influence of molecular diffusion. That is presented by exhibiting lower concentrations at the same distances from the source, e.g. for the third row of Fig. 5.17, most of the mass still exhibits concentration values greater than $0.5 \mathrm{mg} / 1$ for the case of constant mean velocity (left), while the attenuated case has already switched most of the mass below $0.5 \mathrm{mg} / 1$. These properties are even clearer when cumulative distribution of the corresponding EMFs is given for the chosen limit value of concentration $c^{*}=1 \mathrm{mg} / 1$.

The contaminant mass fraction exhibiting concentration above limit value, as shown in Fig. 5.18, directly indicates dilution intensity since it delivers the distance (or area) at which an arbitrary acceptable level of concentration reduction is achieved.

It is interesting to comment on the behavior of the curve defined for the lowest 
steady mass flux $(30 \mathrm{~g} / \mathrm{s})$. The most of the initial concentration values for that case are below the limit $\left(c^{*}\right)$, but in the near source distances the concentration variance growth is faster than concentration mean reduction, causing spreading of the Beta EMF (see Fig. 5.17 left panel, up, for $x=50,100$, and $200 \mathrm{~m}$ ). This causes the cumulative distribution (Fig. 5.18) to have a slight growth in the near source area, but after that it follows an exponential reduction almost parallel to the curves of higher values of mass loading. It can be concluded that a lower steady mass flux is more influenced by source characteristics at the river mouth, but the final behavior is not compromised. Hence, the contaminant mass fraction exhibiting concentration levels above the limit value (Fig. 5.18) presents a potentially useful indication of the level of achieved dilution. That might be developed into a specific application discussed in Section 5.4.

\subsection{Measurements}

The procedures used for velocity and salinity measurements were previously described in detail in Chapter 4. This section presents several characteristic results.

\subsubsection{Velocity measurements}

The focus of velocity measurements, as explained in Section 4.2, was to determine the mean velocity profile in the downstream direction. Since an assumption of depth integrated velocity and its dominating downstream direction was made in analytical modeling, it was tested in the field. The proposed exponential form of mean velocity attenuation in dependence to the $x$ axis (2.17) was a result of measured velocities. The taken measurements were analyzed and organized in a separated form to distinguish those with positions on or off the centerline. (Fig. 5.19). Those off the centerline were more influenced by bathymetry and riverbanks, which were converted into an unregulated type of beach groynes. Afterwards, the centerline values are fitted with the proposed exponential velocity model and shown in Fig. 5.20. The first set of velocity data demonstrates a good agreement with the proposed depth-averaged velocity model (2.17) with the attenuation coefficient $\nu=0.002 \mathrm{~m}^{-1}$, while the second set suggests that a nearly constant mean velocity $(\nu=0.0)$ is established near the river mouth due to the higher river flow rate, which was present in that measurement campaign. Also, the proposed mean velocity model was further tested in Section 5.4 against the data obtained by the other analytical model and MOHID numerical data. 


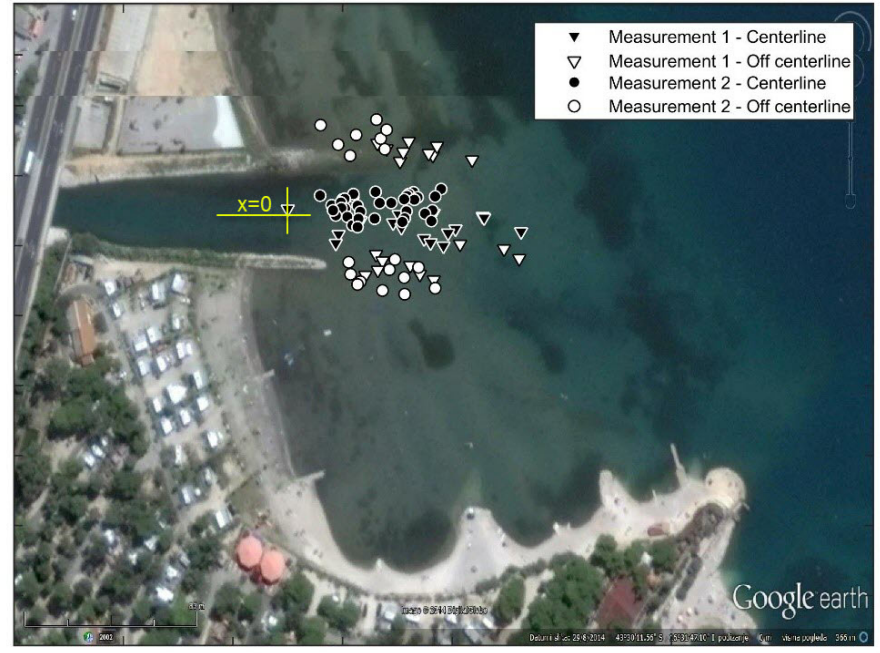

FIGURE 5.19: The positions of velocity measurements used for the evaluation of the velocity attenuation coefficient.

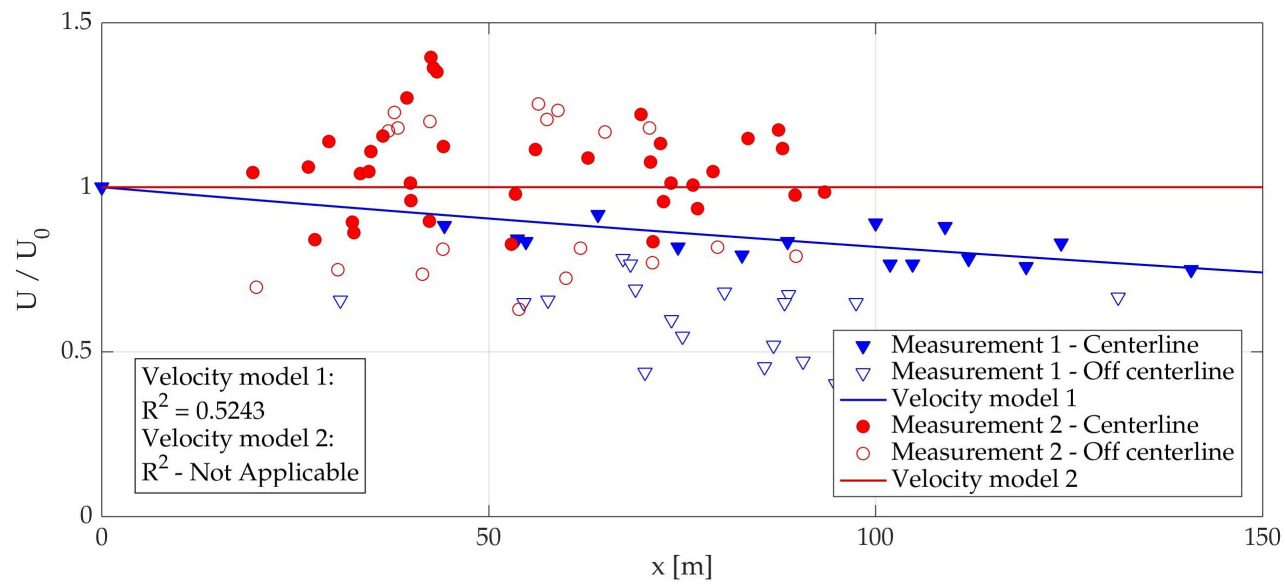

FIGURE 5.20: Measured velocities fitted with the mean velocity model provided by (2.17); model1: $U(x)=0.4 e^{-0.002 x} \mathrm{~m} / \mathrm{s}$, model2:

$$
U(x)=0.6 \mathrm{~m} / \mathrm{s} \text {. }
$$

The first two field measurement campaigns focused on obtaining only mean velocity; however, salinity was also measured at several points to determine whether stratification was present.

Salinity measurements clearly show a salt-wedge stratification (Fig. 5.21), with the less dense freshwater contained in the upper, 30-centimeter thin layer, while salt water was confined in the underlying salt wedge, as typically occurs in similar estuaries $[123,86]$. The salinity stratification data is in accordance with the measured velocities, suggesting that pollutants are mainly transported in the upper layer that can be characterized by a spatial field of depth-averaged velocities. 


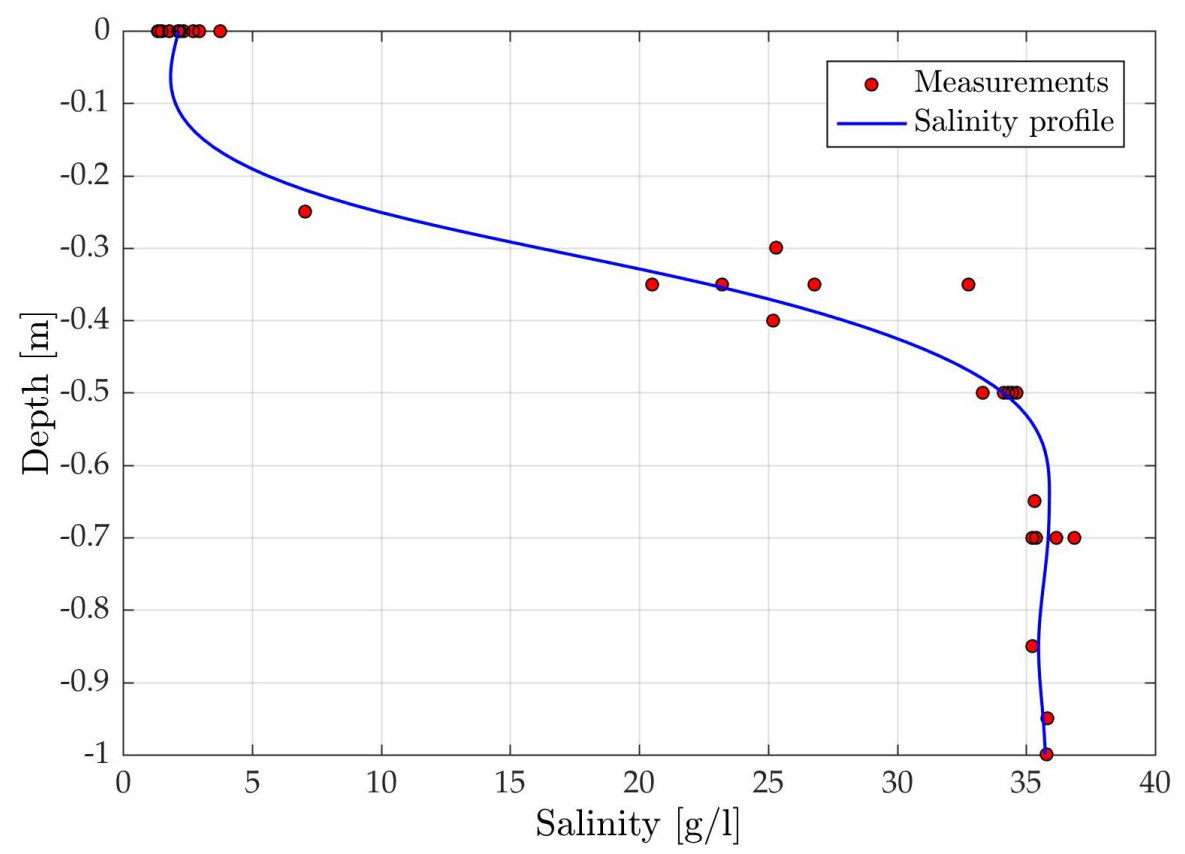

FIGURE 5.21: Estimated mean from salinity measurements taken at 10 locations near the river mouth.

The obtained velocity data showed a dominant role of stream flow velocity, as velocities measured perpendicular to the plume axis $x$ were negligible. Most of the flow was carried in an upper shallow layer of freshwater $(10-30 \mathrm{~cm})$, which is confirmed by the detected salt wedge (Fig. 5.21). Velocities for points deeper than $30 \mathrm{~cm}$ with respect to the water surface were undetectable and thus neglected.

\subsubsection{Salinity measurements}

When the CPoRT project was launched in 2015, an inflatable boat and a more accurate GPS device were purchased as additional equipment, which enabled more extensive measurement campaigns. The idea of validation switched from velocity to salinity measurements as explained in Section 4.2 , where salinity is used as a proxy for normalized solute concentration in the flow surface layer (Eq. (4.1)). To give an adequate representation of measurement results, example data was given for each measurement campaign and for chosen measurement points, which were annotated in Fig. 4.10. All salinity data ( $S$ [PSU]) was converted to nondimensional proxy concentration defined by Eq. (4.1) $\left(c_{p}\right)$.

\section{First measurement campaign}

The first measurement campaign was conducted using a 10-second sampling period for 2 minutes of recording per point, resulting in a rather small number of 
samples. However, the 55 recorded points managed to deliver a part of surface mean proxy concentration and surface variance when linear interpolation was applied (Fig. 5.23). In Fig. 5.22, two characteristic points (one close to the river mouth and one further downstream) are presented with the corresponding proxy concentration measurements and the probe position fluctuations during the measuring time.
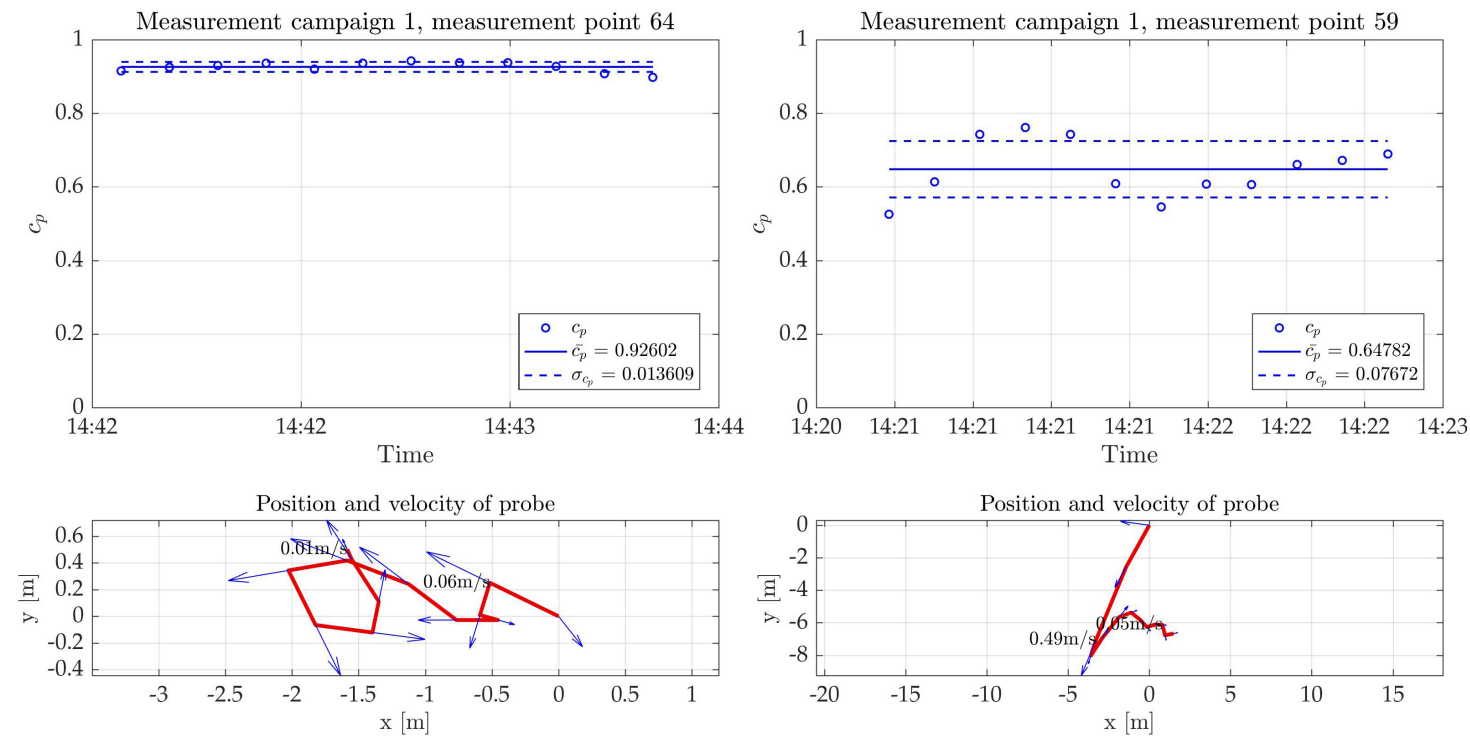

FIGURE 5.22: Proxy concentration $\left(c_{p}\right)$ measured at two characteristic points for the first measurement campaign with annotated probe movement.
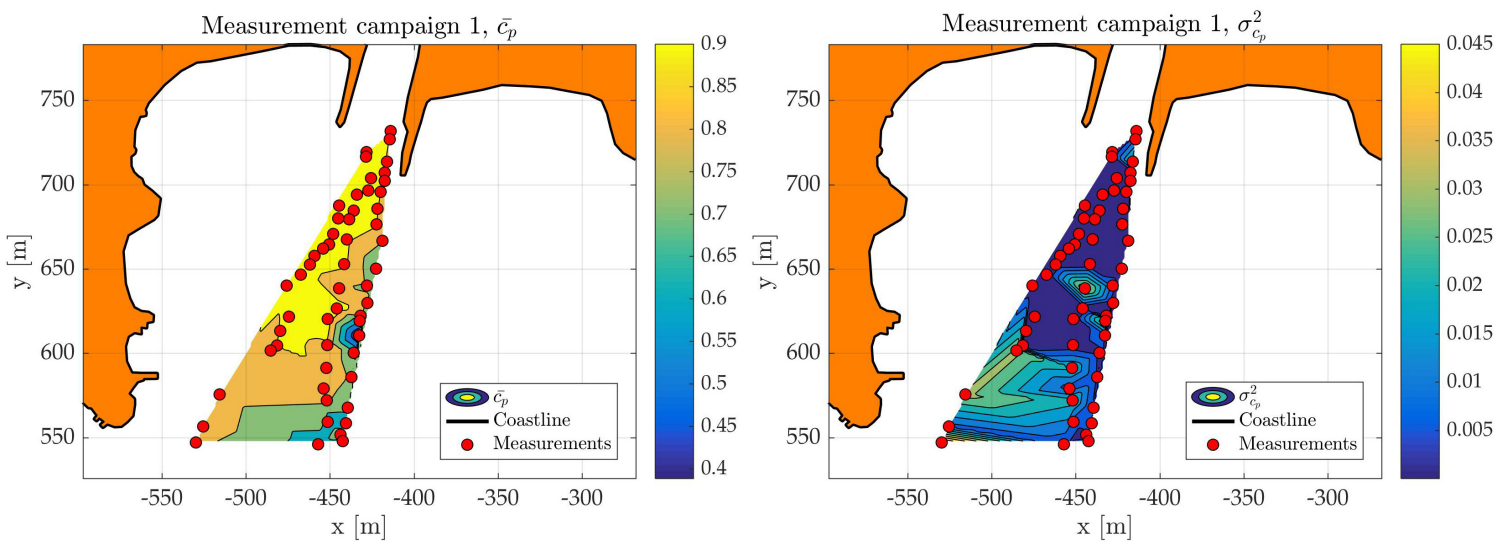

FIGURE 5.23: Proxy mean concentration and variance field for the first measurement campaign obtained by linear interpolation of measurement points.

\section{Second measurement campaign}

The second measurement campaign was conducted using a 6-second sampling 
period again for 2 minutes of recording per point, resulting in a rather small number of samples. Since field conditions were better and the procedure was more optimized than the first time, a larger number of points (105) was recorded which enabled a better qualitative description of proxy mean concentration and variance field (Fig. 5.25). Again, two example points are given to represent the proxy concentration behavior at the river mouth and at a more distant position in Fig. 5.24.
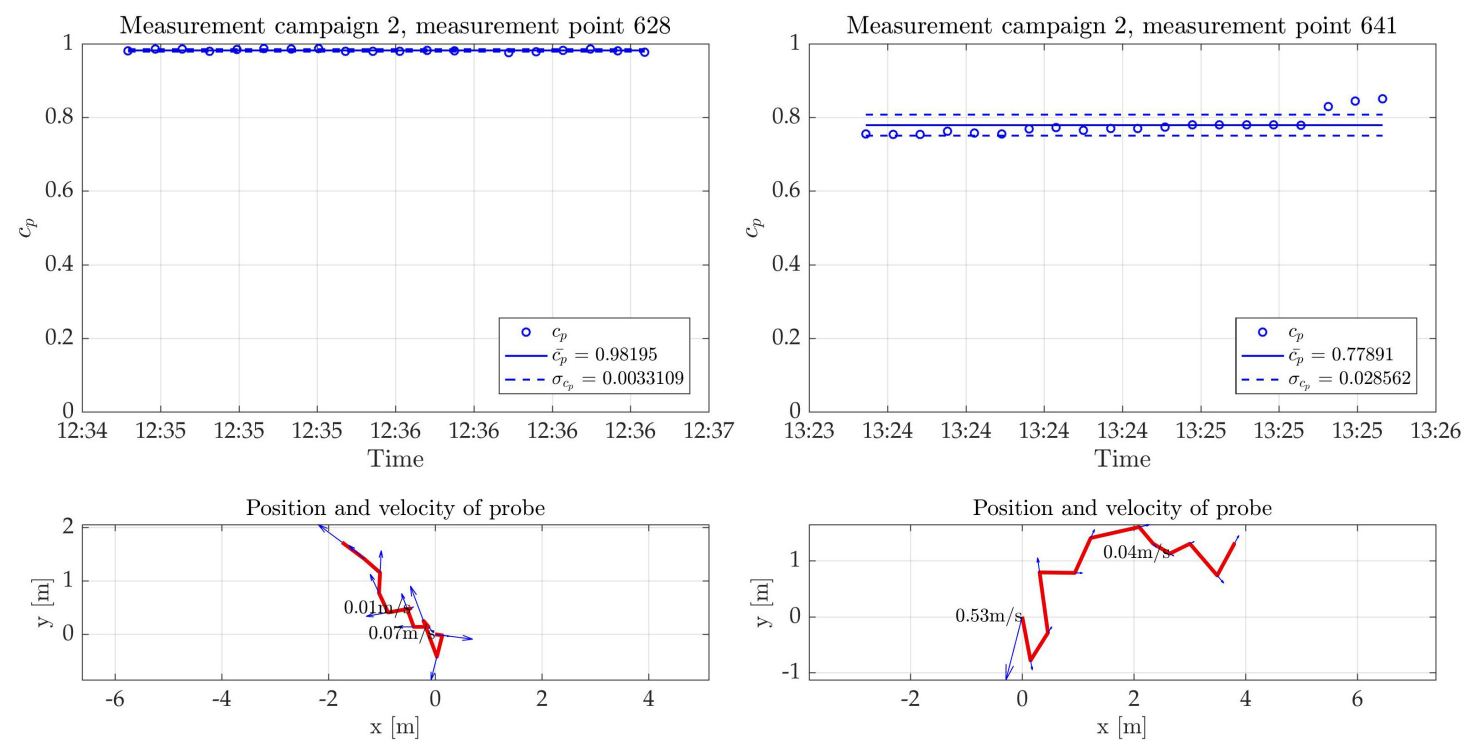

FIGURE 5.24: Proxy concentration $\left(c_{p}\right)$ measured at two characteristic points for the second measurement campaign with annotated probe movement.
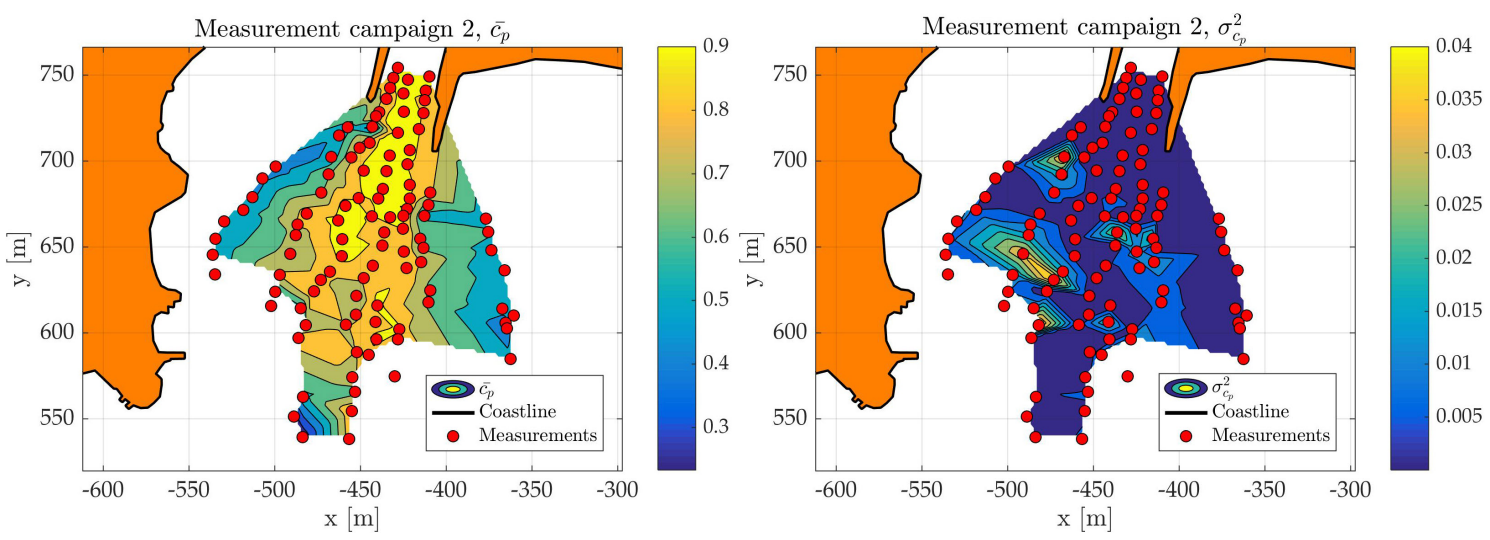

FIGURE 5.25: Proxy mean concentration and variance field for the second measurement campaign obtained by linear interpolation of measurement points. 


\section{Third measurement campaign}

The first and particularly the second measurement campaigns were more focused on obtaining a general qualitative description of proxy concentration mean and variance field in the area of interest. However, the level of statistical analysis which may be achieved with only 12 samples per point in the first campaign, and 20 samples in the second one, was questionable. Therefore, the third measurement campaign (Fig. 5.26 and Fig. 5.27) was conducted also with a 6-second sampling period, but with a recording interval of 30 minutes per point.
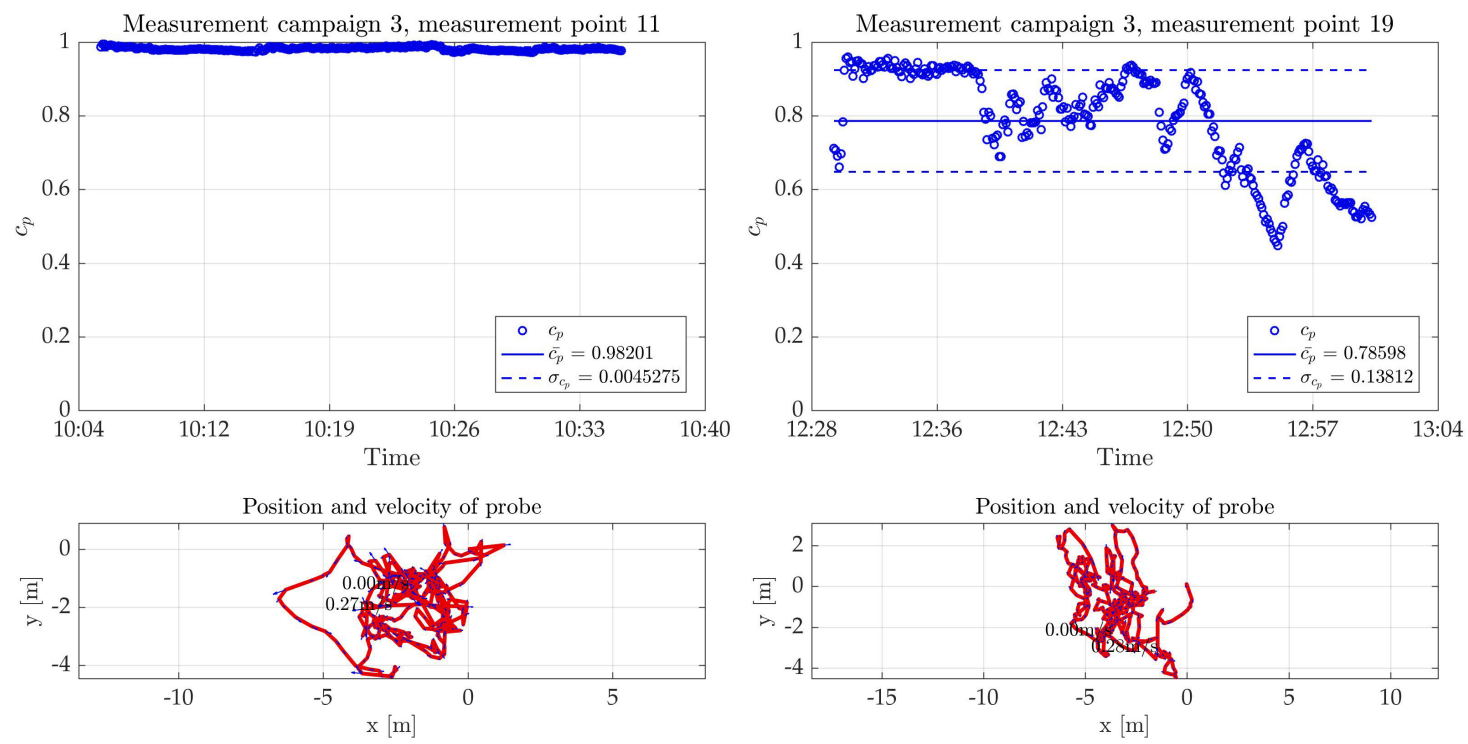

FIGURE 5.26: Proxy concentration $\left(c_{p}\right)$ measured at two characteristic points for the third measurement campaign with annotated probe movement.
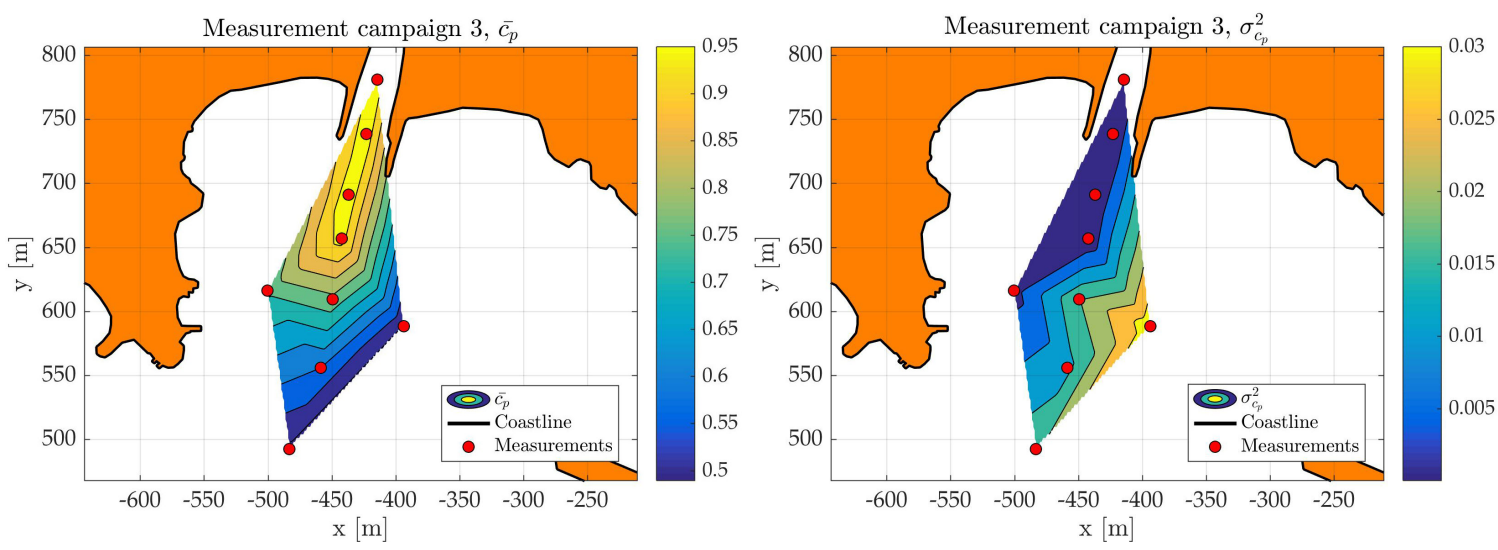

FIGURE 5.27: Proxy mean concentration and variance field for the third measurement campaign obtained by linear interpolation of measurement points. 
Naturally, a small number of points was obtained (only 9), but the 300 samples per point have shown good statistical inference, which is represented by the obtained PDFs defined at three different points. These PDFs demonstrated expected behavior, which can be seen in Fig. 5.28. For instance, the PDF at the initial point (measurement point 11) is still practically inside the river and has almost only freshwater or, to put it differently, $c_{p} \approx 1.0$. The values become more scattered along the downstream distance, which is why a bell shaped Beta distribution is present at measurement point 19 due to increased variance. However, measurement point 17 exhibits lower values of variance and, although the concentration is lower off the centerline, the distribution shape is narrower.
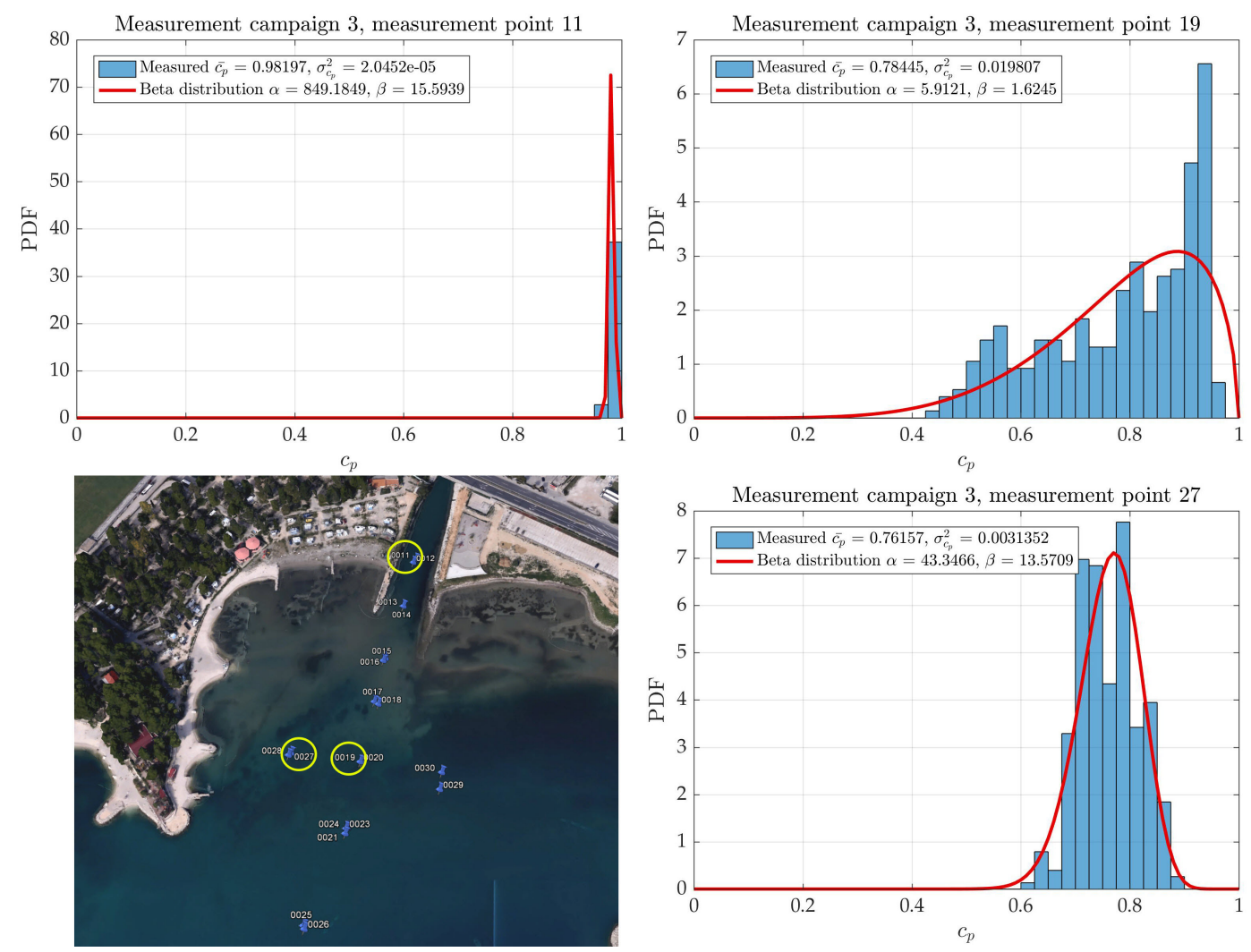

FIGURE 5.28: PDFs of proxy concentration for 3 example points from the third measurement campaign shown on a corresponding map.

The data from the third measurement campaign was also used to deliver an estimate of an acceptable measuring interval required for obtaining convergence of proxy concentration and variance. In accordance with the presented convergence (Fig. 5.29), a 10-minute interval was chosen to be applied in the fourth measurement campaign, since it had shown an acceptable level of convergence 
while preserving the possibility of covering at least 15 points per measurement campaign.
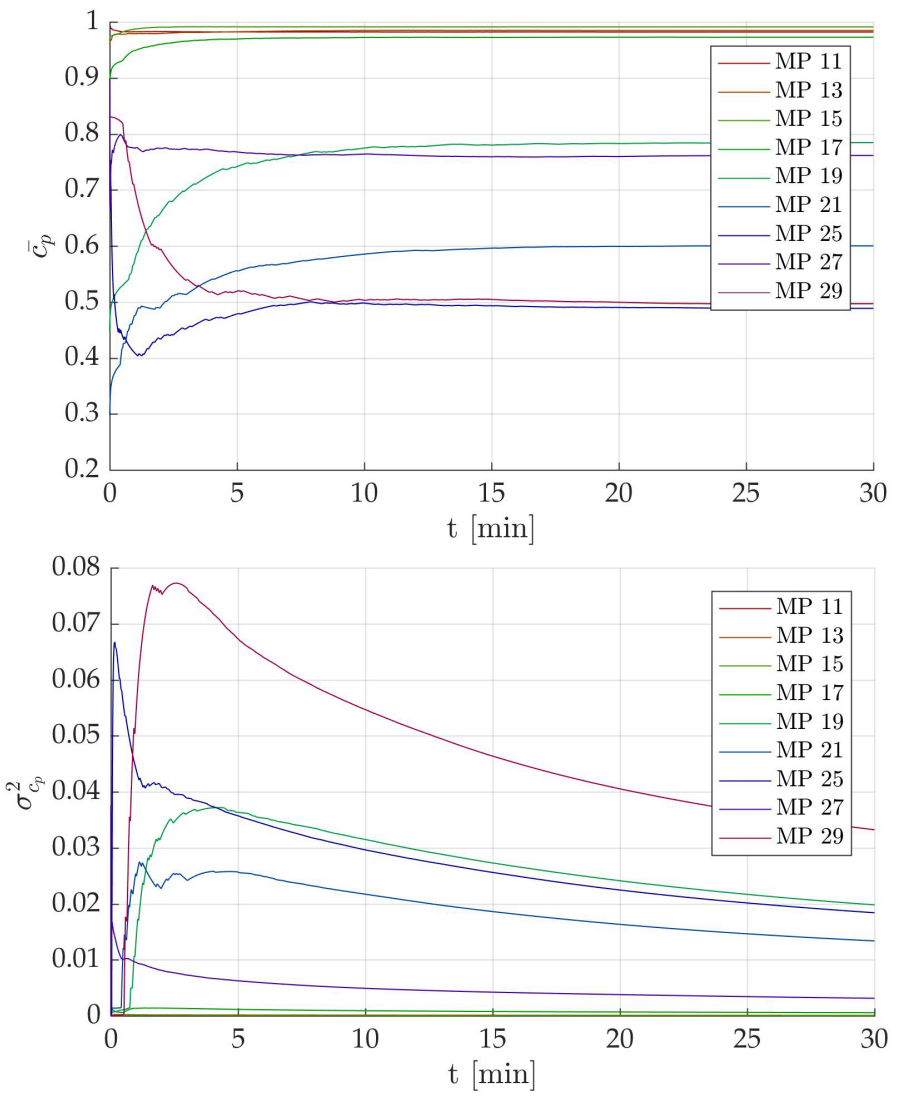

FIGURE 5.29: The convergence of proxy concentration statistics relative to the measuring interval.

One may notice the mean concentration being converged even somewhat before 10 minutes, while variance still tends to fluctuate. The convergence diagrams for all measurement points in Fig. 5.29 are obtained by listing the mean and variance values in a descending order for all 300 samples obtained within 30 minutes of the measuring interval.

\section{Fourth measurement campaign}

As mentioned above, these measurements used a 10-minute recording interval resulting in approximately 100 samples per point. Since this kind of measurement could not provide many points, it was used to obtain transversal profiles in the best possible way. These profiles are presented in Fig. 5.32, while a similar structure of two example points and surface proxy mean concentration and variance, as defined for other measurement campaigns, are shown in Fig. 5.30 and Fig. 5.31, respectively. 

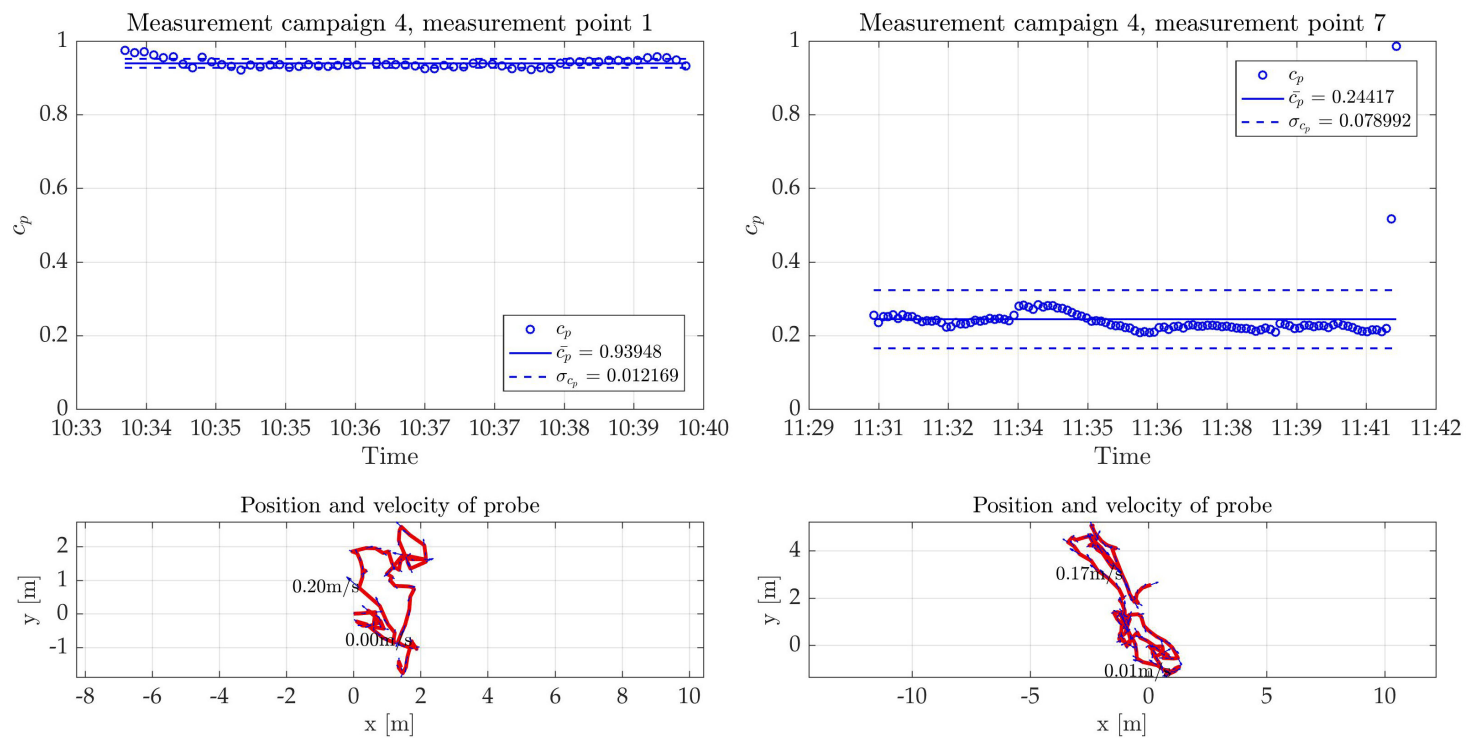

FIGURE 5.30: Proxy concentration $\left(c_{p}\right)$ measured at two characteristic points for the fourth measurement campaign with annotated probe movement.
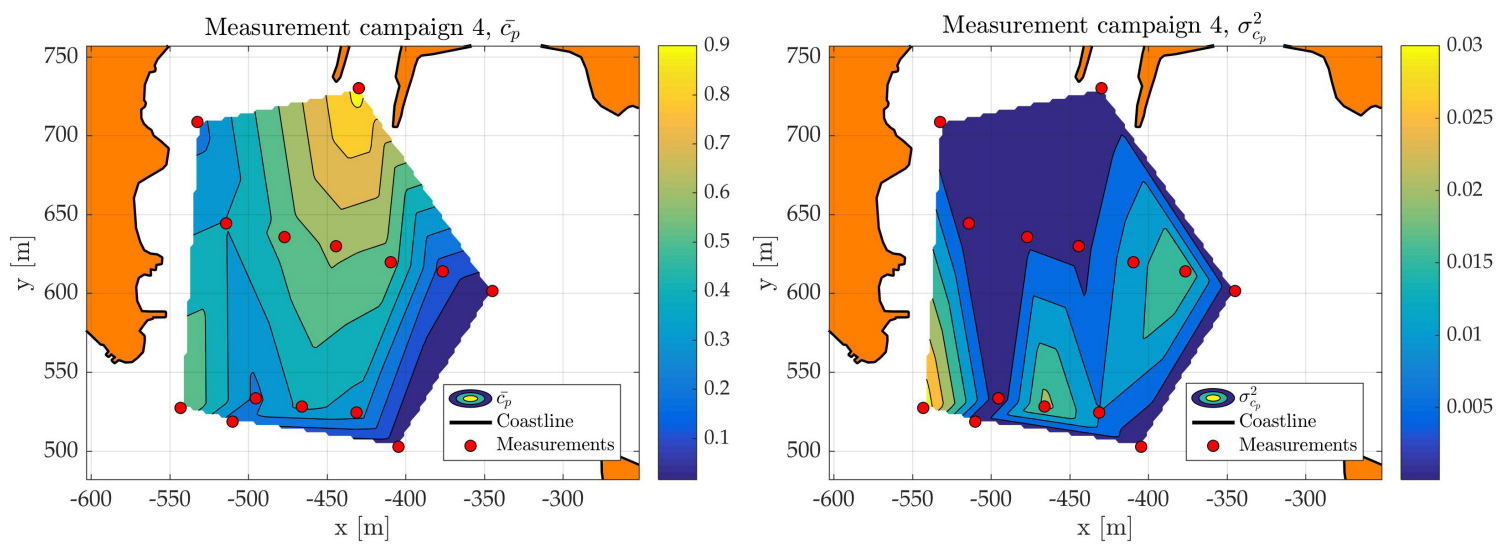

FIGURE 5.31: Proxy mean concentration and variance field for the fourth measurement campaign obtained by linear interpolation of measurement points.

Also, the fourth campaign is important since the proxy concentration moments defined in points for these approximately transversal profiles will subsequently be used for defining the corresponding EMFs when a comparison is given in Section 5.4 . 

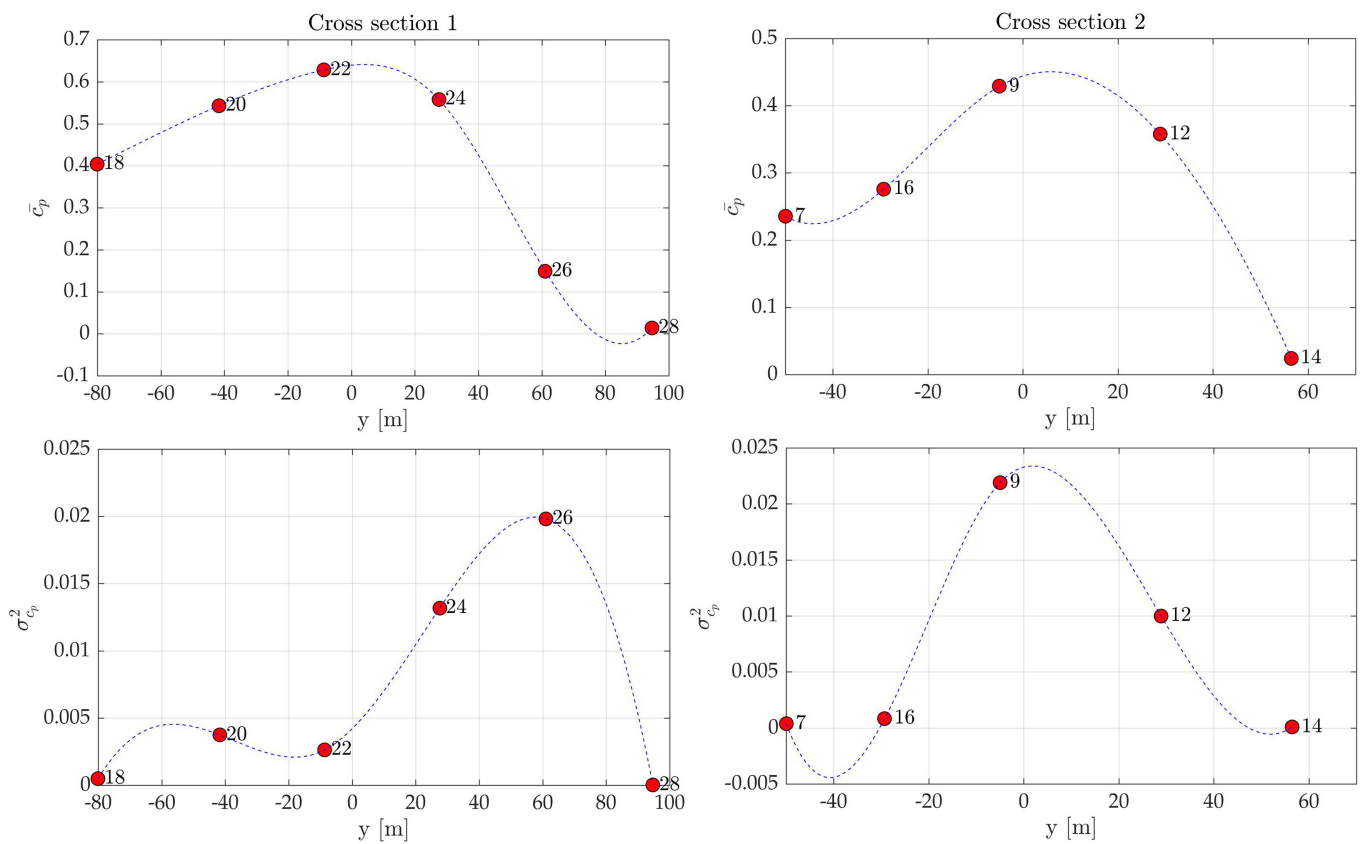

FIGURE 5.32: Proxy mean concentration and variance at two downstream distances from the source; $x=93 \mathrm{~m}$ (left) and at $x=200 \mathrm{~m}$

(right).

In conclusion, each measurement campaign had its own specific goal, where the first one was mostly preparation, the second one was used to obtain good qualitative representation of the proxy concentration field in the area of interest, while the third and fourth campaigns gave better statistical inference due to a higher number of samples per point. For instance, the surface plots for proxy mean concentration and variance were obviously not well represented with these few points for both the third and the fourth measurement campaign. However, additional velocity measurements, which were conducted simultaneously with salinity measurements in the third campaign, were compared to MOHID data and the proposed analytical model (Section 5.4). Furthermore, proxy concentration statistics obtained in the third campaign were used in combination with the qualitative layers of the second campaign to produce a comparable surface plot with the results of analytical and numerical (MOHID) modeling (Section 5.4).

\subsection{Numerical modeling}

As explained in Chapter 4, the numerical modeling was used in order to achieve certain level of verification for developed analytical methodology. This section 
delivers the example results for using RWPT model and MOHID model. However, the different approaches for comparisons of results from analytical and numerical modeling are part of the Section 5.4.

\subsubsection{Random walk particle tracking model}

The implementation of RWPT for the case of steady conservative transport which resembles that of an analytical model was explained in Chapter 4.
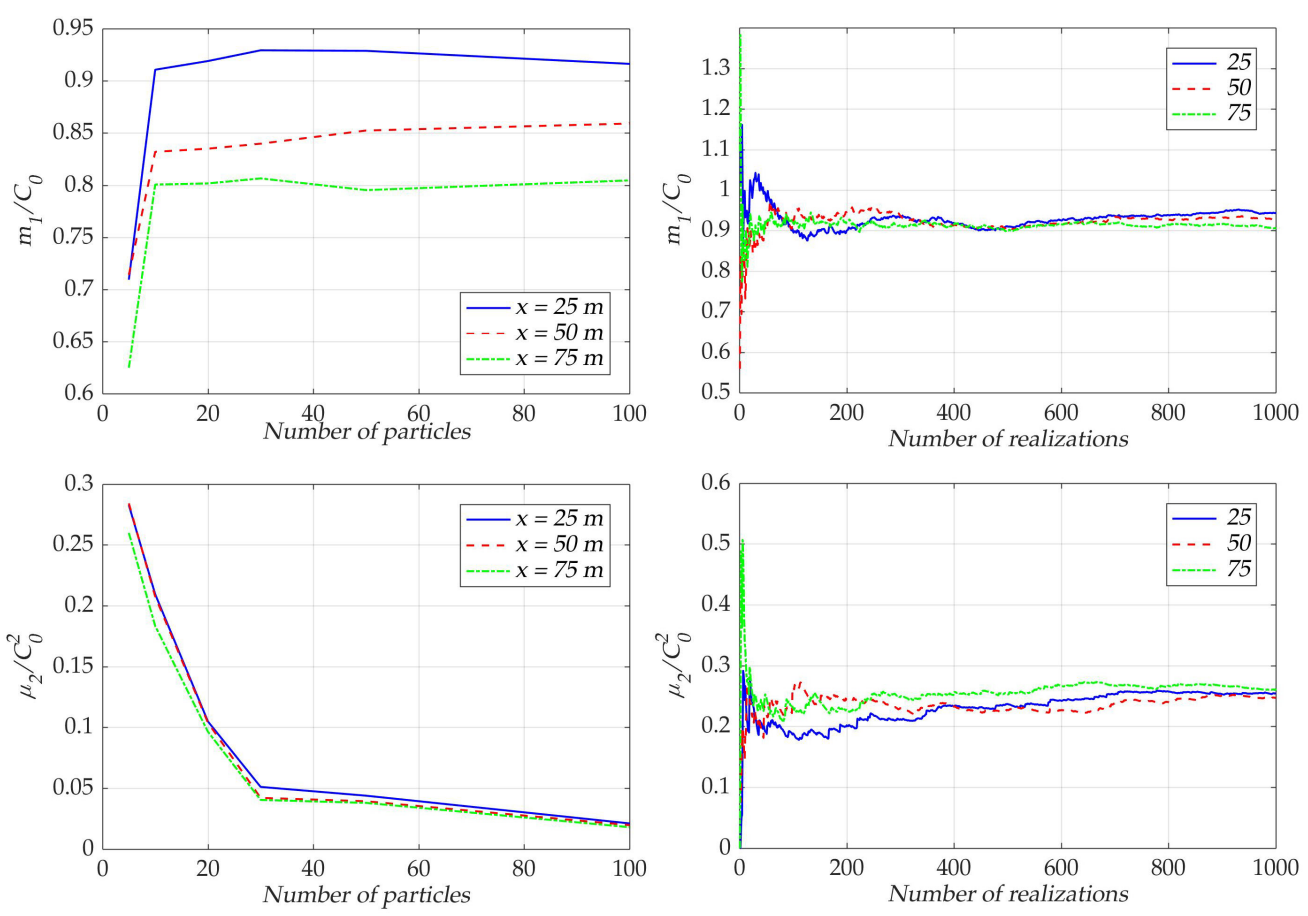

FIGURE 5.33: Convergence tests for the RWPT model with regard to the number of particles introduced in each time step (left) and the number of realizations (right).

The RWPT model was tested for convergence levels, with regard to the number of particles introduced in each time step and the number of realizations. Hence, the first two moments, which were obtained for normalized concentration values defined by Eq. (4.5), were calculated at different cross sections downstream (see Fig. 5.33). It may be noticed that the mean is quite converged at even 20 particles or less; however, variance exhibits more stable behavior at 30 particles and above. That is even more pronounced when considering the number of realizations, since higher moments always require more realizations. Both mean and variance get stabilized at around 600 realizations. Nevertheless, since the computational time of the model was more influenced by the number of particles than the number of realizations, results were obtained with 1000 realizations. 

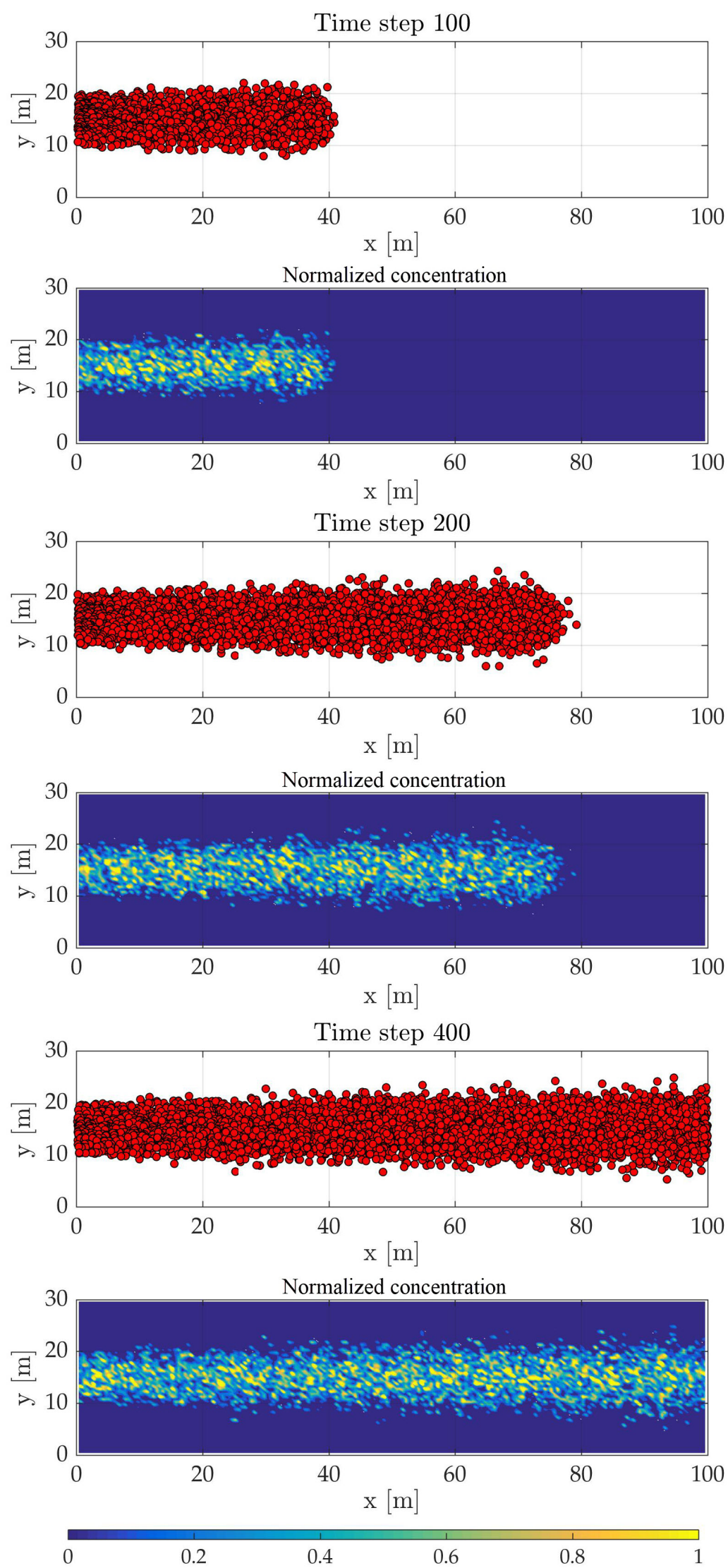

FIGURE 5.34: Different snapshots (for $t=100,200,400 \mathrm{~s}$ ) of an arbitrary RWPT realization. 
A visualization of three different time steps of an arbitrary realization is given in Fig. 5.34. The duration of each realization was defined by the condition of reaching a steady state, when the monitored difference between the number of particles entering and exiting the system is below the defined limit (Section 4.3.1). However, most realizations reached those conditions at approximately 400 steps.
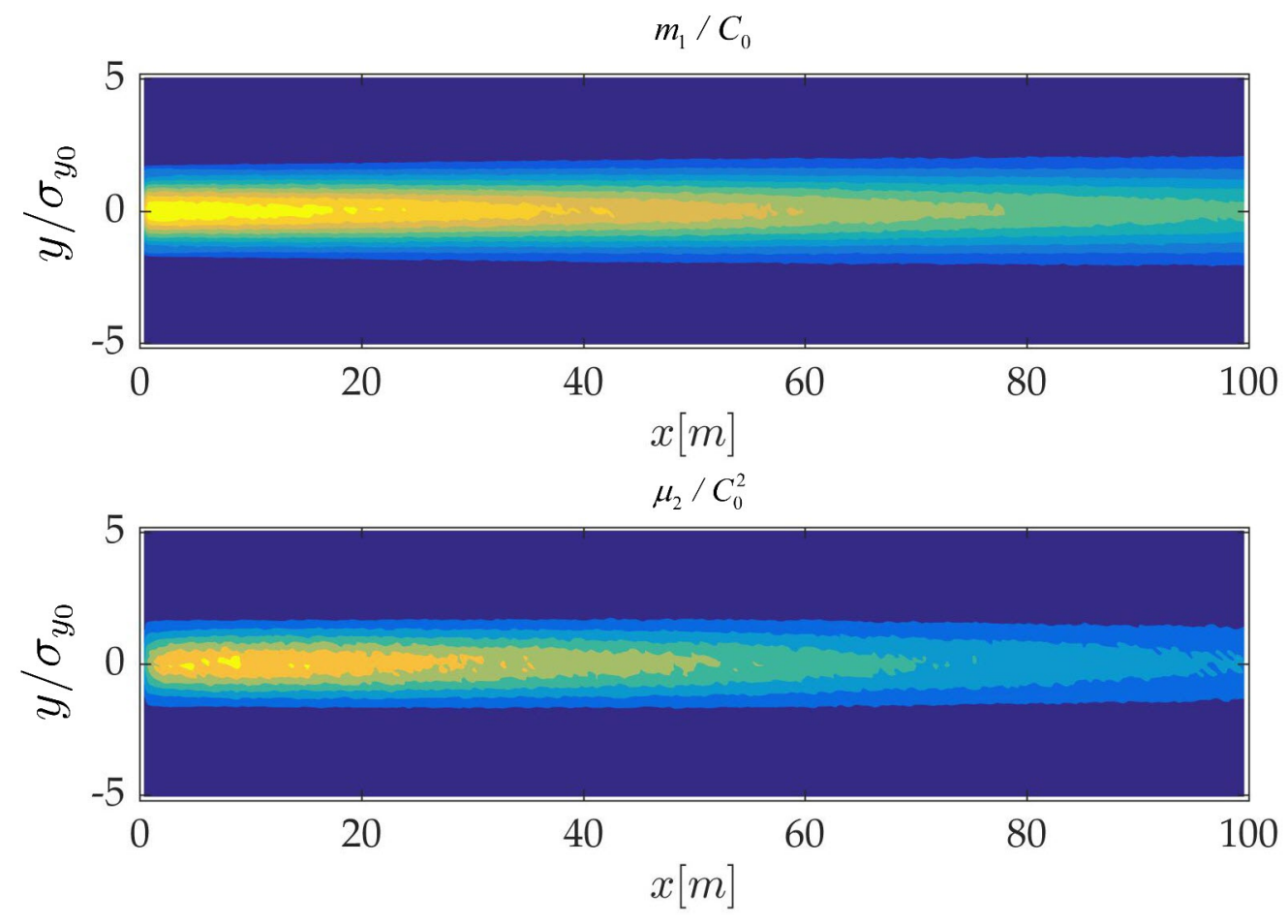

FIGURE 5.35: Final concentration mean and variance for all domains after 1000 realizations.

Finally, surface plots for both the concentration mean and variance are presented in Fig. 5.35.

\subsubsection{MOHID}

The MOHID model was implemented in cooperation with the MARETEC research team [96] from Lisbon in order to investigate further potential validation of the analytical model. It was used for the simulation of the field conditions at the time of third measurement campaign (December 29, 2015) which enabled verification of proposed mean velocity model (Eq. (2.17)) and increased the spatial resolution of data. As previously described in Chapter 4, both the 2D and 

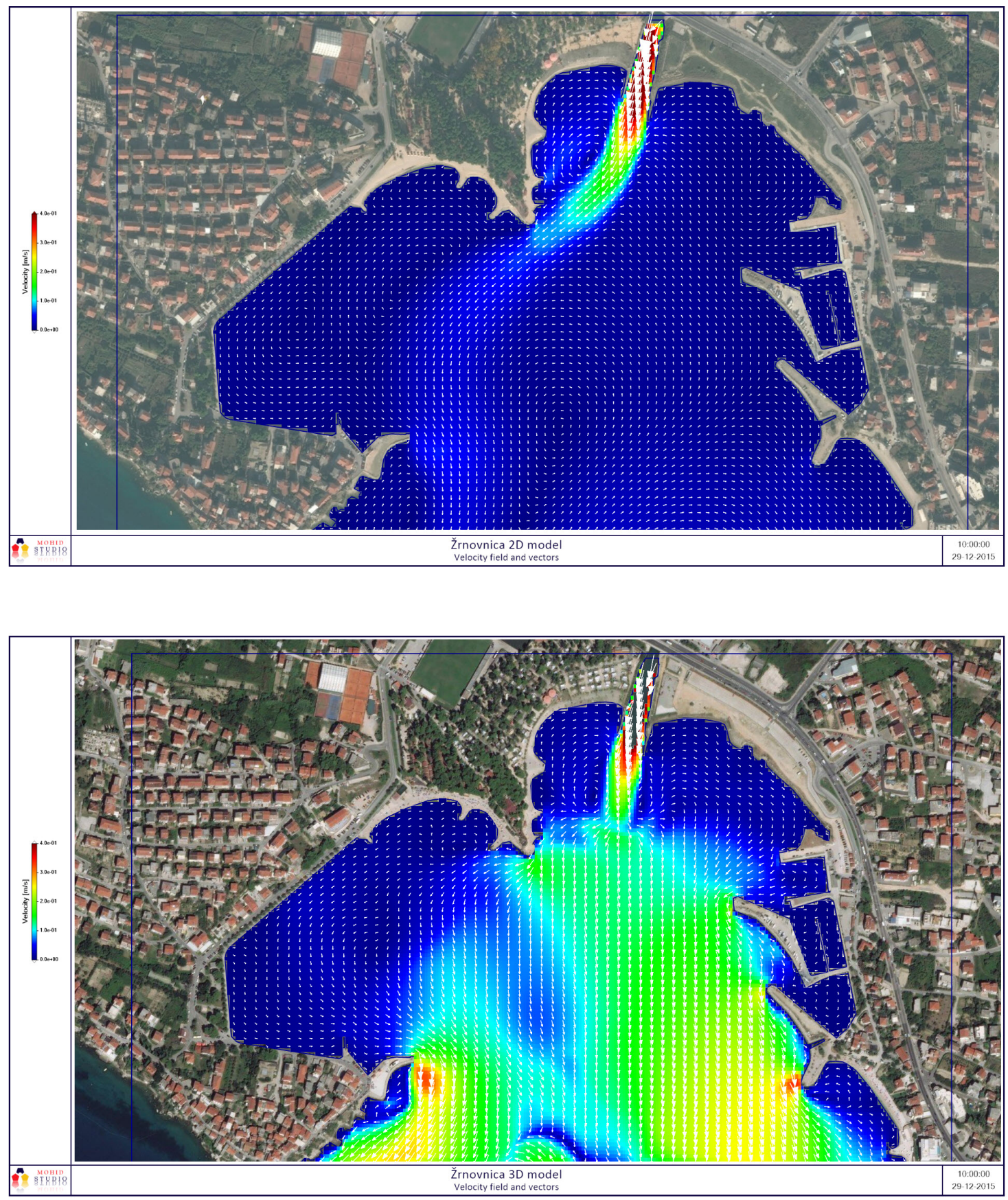

FIGURE 5.36: A snapshot of MOHID results for the velocity field using a 2D model (up) and a 3D model, top layer (down).

the 3D model were made for the study case of the Žrnovnica River and the compared results are presented here for the velocity field (Fig. 5.36), the salinity field (Fig. 5.37), and the results of Lagrangian transport (Fig. 5.38).

For the initial modeling step, a simple 2D model was developed, which cannot simulate stratification. Due to the stated nature of the 2D model, the results for the velocity field, salinity, and Lagrangian transport were all dominated by 

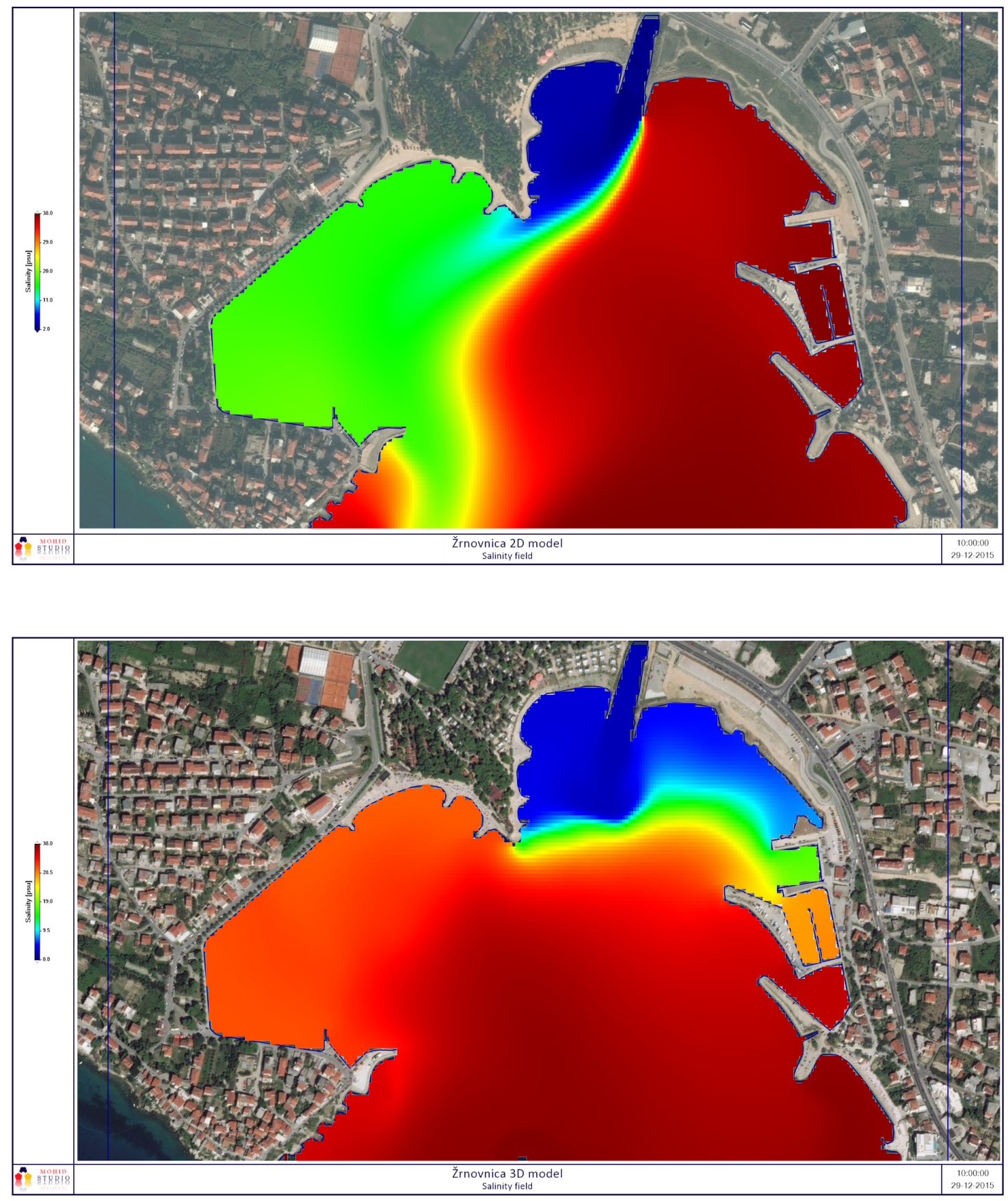

FIGURE 5.37: A snapshot of MOHID results for the salinity field using a 2D model (up) and a 3D model, top layer (down).

bathymetry. This effect is directly seen in the 2D part of all presented figures, where all characteristics are swayed away in the left part of the bay, which follows the deeper area (Fig. 4.2).

When a 3D model was implemented, the salinity wedge (see Fig. 5.39) was simulated and it demonstrated more accurate behavior, since a similar wedge was detected by measurements (Fig. 5.21). 

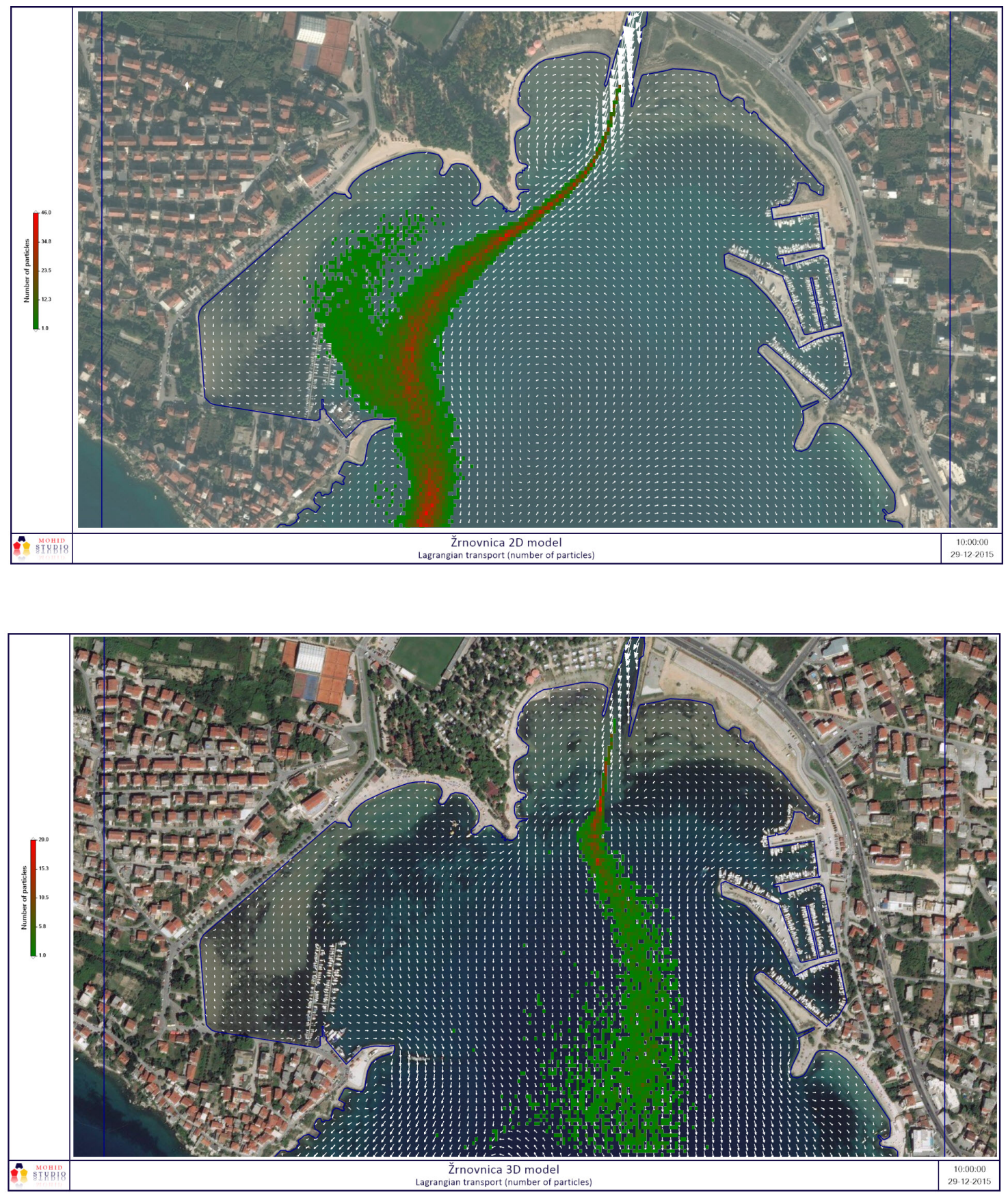

FIGURE 5.38: A snapshot of MOHID results for Lagrangian transport using a 2D model (up) and a 3D model, top layer (down).

The centerline of the flow coming to the river is much less affected, since it slides in the upper layer of the stratified flow. However, due to the inertia of the denser body of salt water and a more intense depth change, the river flow tends to get stuck in approximately the first $300 \mathrm{~m}$ from the coastline. Salt water in the lowest layer does not get to fully intrude that area, which indicates a combined effect of river flow domination and bathymetry properties. All results are given 

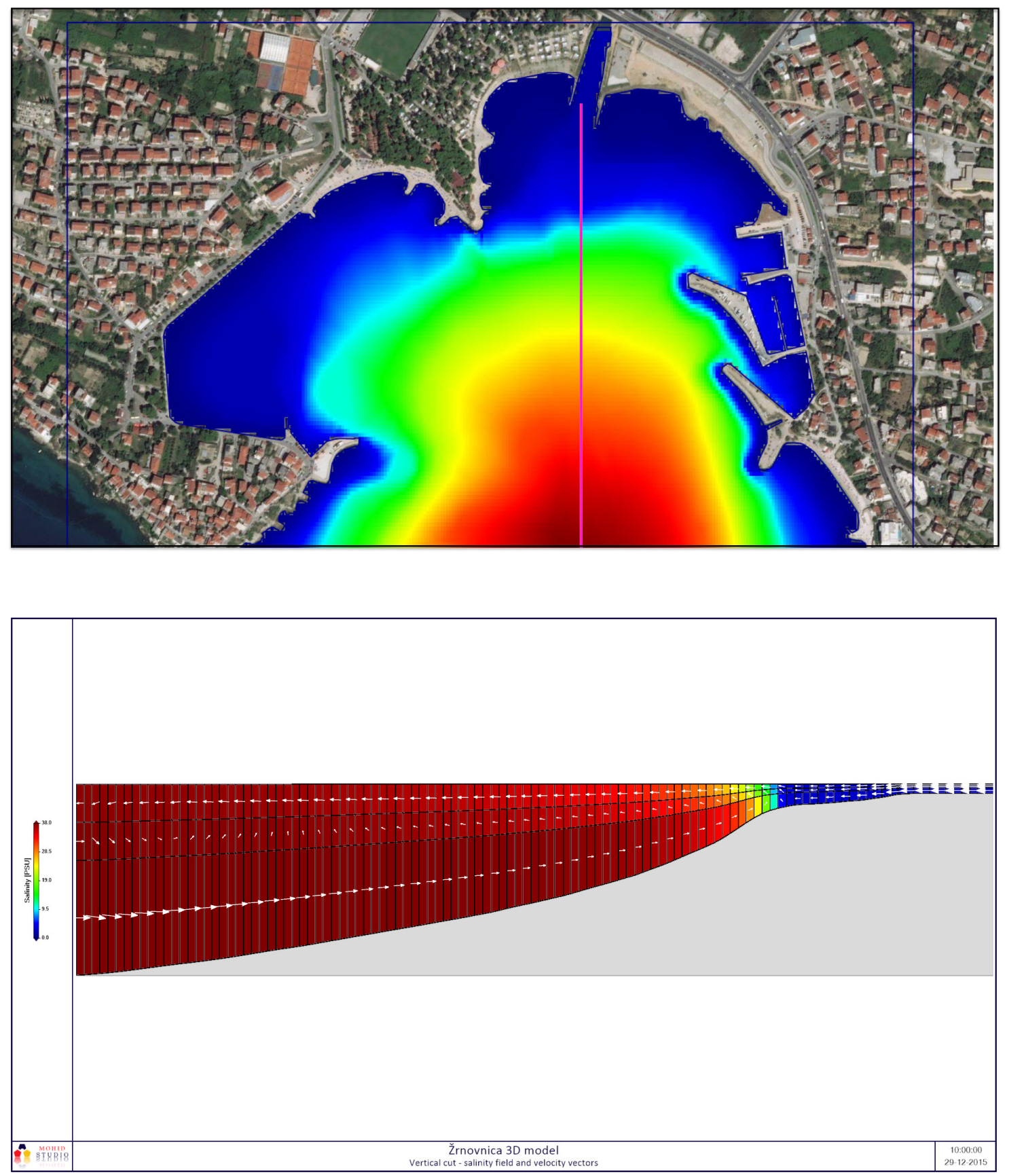

FIGURE 5.39: A snapshot of the vertical profile (down) and the position of the profile (up).

in the same snapshot (December 29, 2015, $10 \mathrm{am}$ ). The results of the 3D model matched well with the measurements for the most of the area that was covered by measurement points, since that area in fact belonged to these first $300 \mathrm{~m}$ (see Fig. 4.10).

However, all MOHID results shown in above figures only represent one realization, and in order to produce an adequate comparison, mean and variance 
should be calculated, which is done in Section 5.4.

\subsection{Comparisons and discussion}

Additional comparisons between analytical and other potential verification methods are investigated in this section, accompanied by a discussion about the significance of the obtained results. A potential application of the EMF concept as a dilution measure in an estuary is highlighted.

\subsubsection{Mean velocity attenuation model}

The proposed exponential attenuation model for mean velocity (Eq. (2.17)) was tested and compared to the hydrodynamic model MOHID, as well as to the existing theoretical model for jet flow [112]. The velocity measurements conducted as part of the third salinity measurement campaign at nine different locations (see Fig. 4.10 and points annotated in MOHID in Fig. 4.16). MOHID results are laid out for eight hours of simulation with a temporal resolution of $5 \mathrm{~s}$. In Figure (Fig. 5.40), both MOHID multiple results and the jet theory model (a solution with constant average depth) are compared to the proposed attenuation model (Eq. (2.17)) for six points at the jet axis or the centerline.

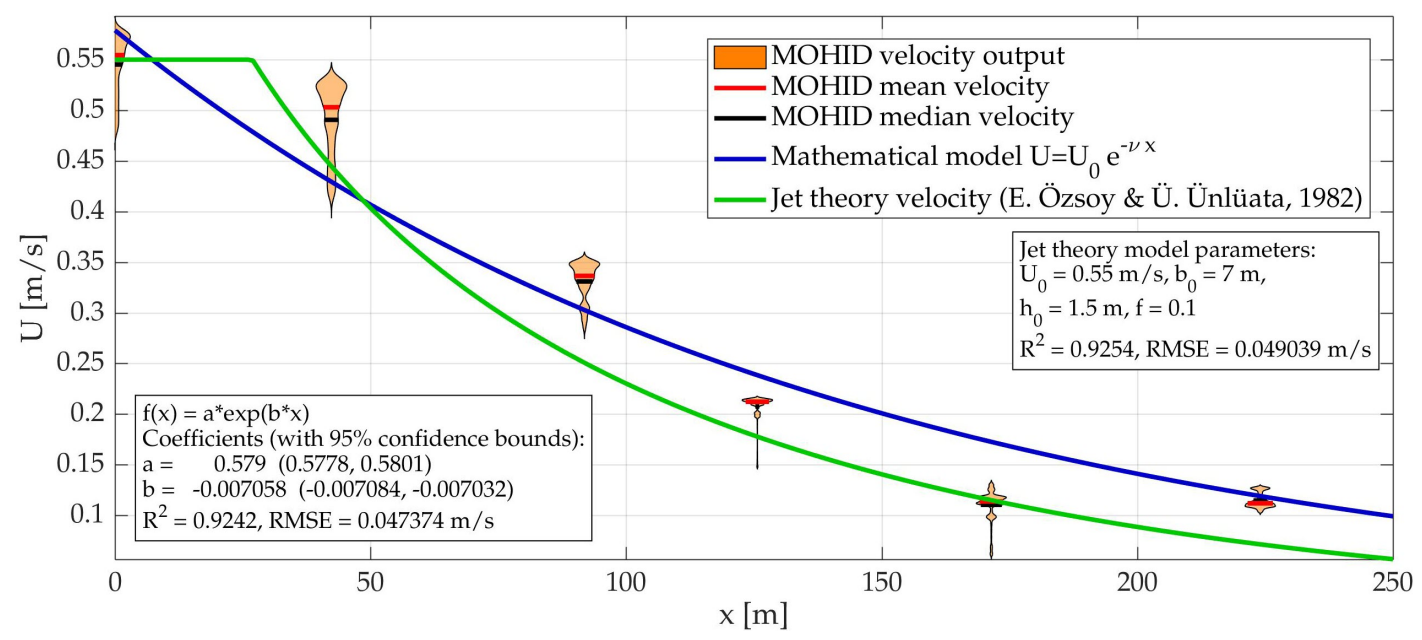

FIGURE 5.40: Comparison between the proposed attenuation velocity model, the jet theory solution, and the MOHID numerical model.

The results presented in Fig. 5.40 suggest that the proposed exponential attenuation mean velocity is a suitable assumption, since it delivers a solution similar 

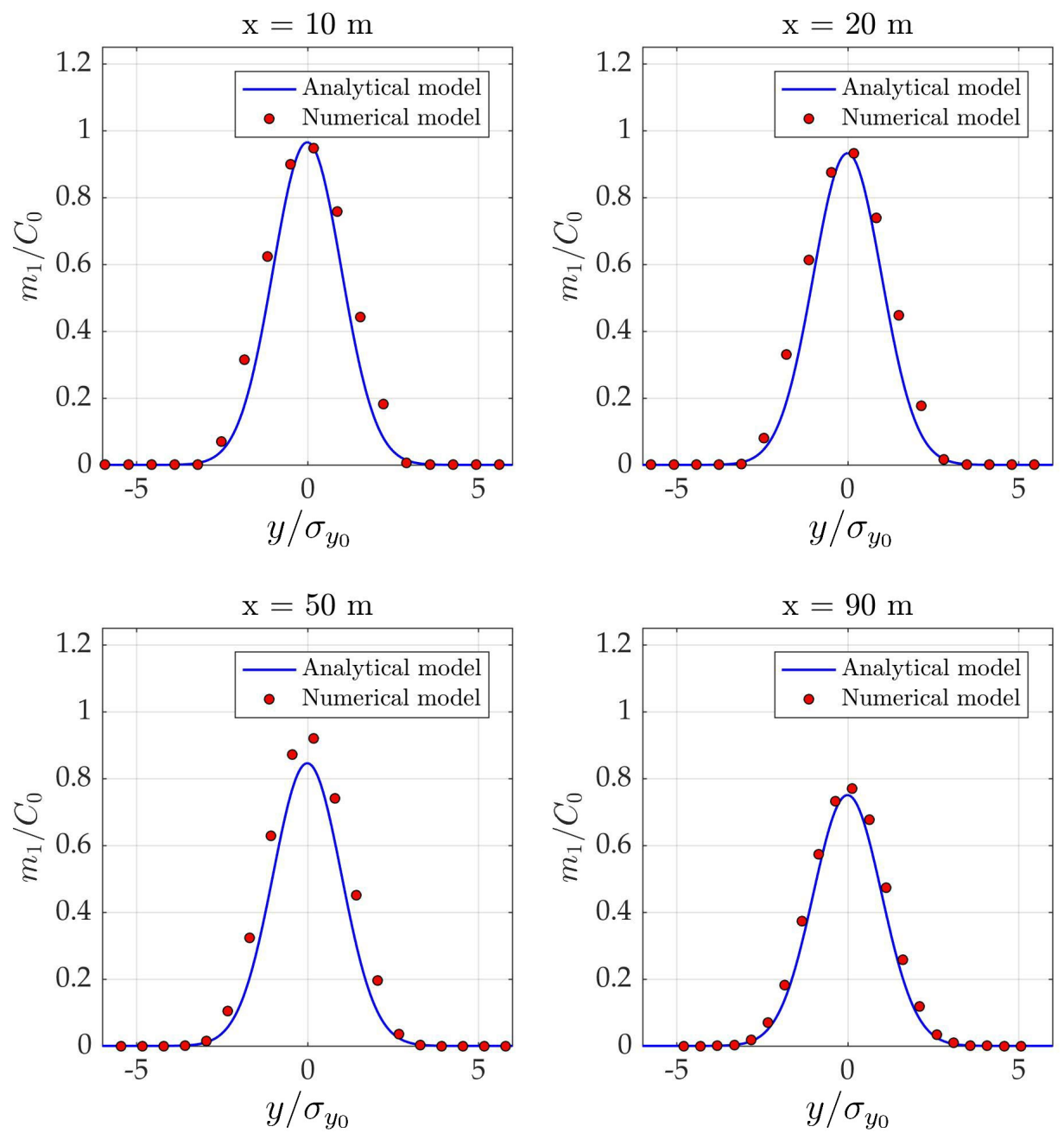

FIGURE 5.41: Comparison of the mean concentration yield by a proposed Gaussian distribution and the numerical model.

to MOHID results and measurements, while obtaining a simple expression to be easily used within the recursive equation for concentration standard moments.

\subsubsection{Comparison of the first two concentration moments between the analytical and the RWPT numerical models}

After 1000 realizations performed by the RWPT numerical model (Section 4.3.1), the concentration mean and variance were obtained on the plane normal to the mean flow and compared to the proposed analytical model.

Fig. 5.41 shows good agreement between numerical and analytical transverse mean concentration distributions at four distances from the river mouth. These results also demonstrate that the concentration mean does not depend on the spatial scale on which the concentration mean is defined, which is supported by 

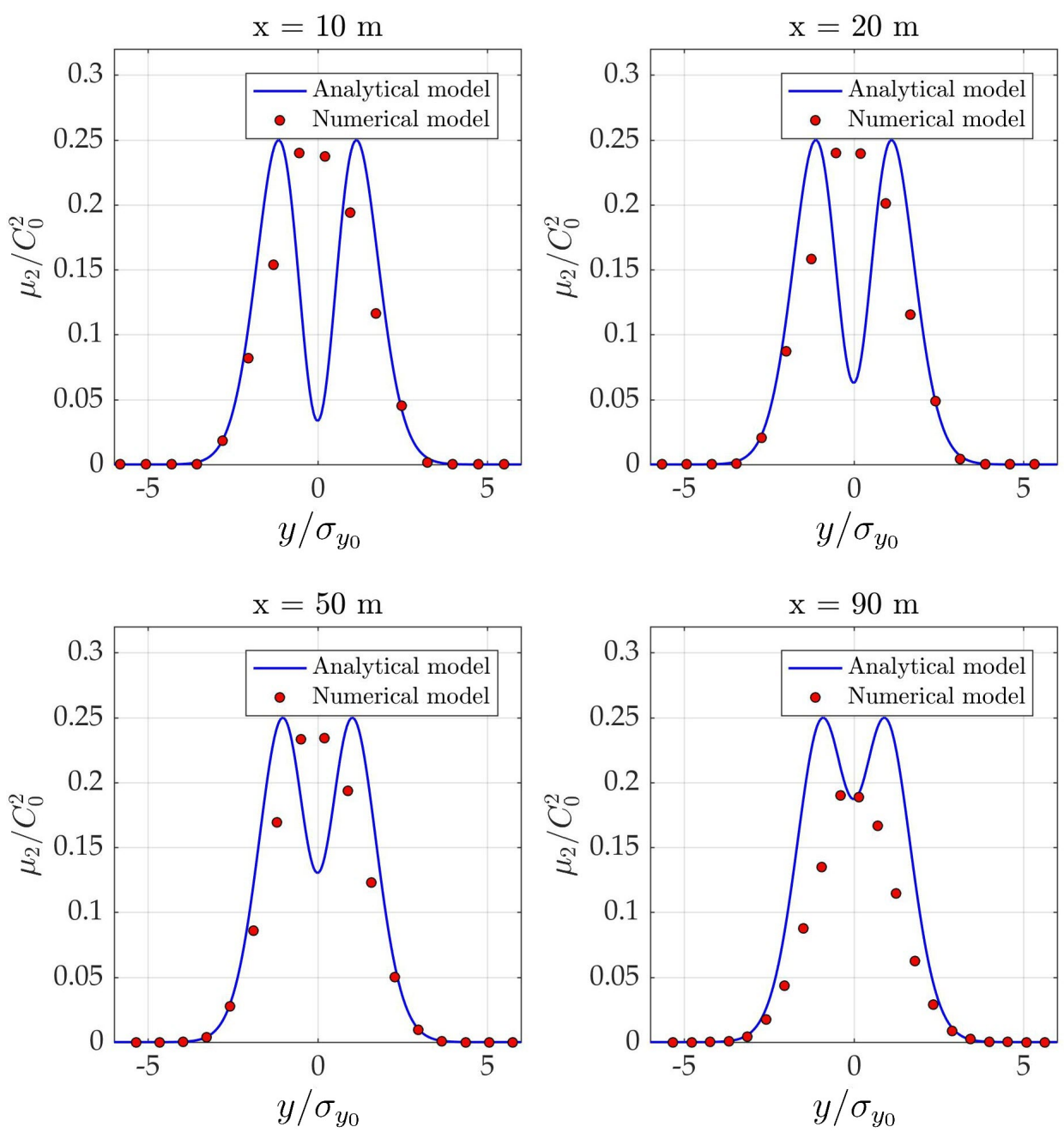

FIGURE 5.42: Comparison of the concentration variance yield by the analytical model (Eq. (2.22)) and the one computed using the RWPT numerical model.

a very good match between the numerically and analytically obtained concentration means in Figure Fig. 5.41. Additional results confirming this statement can be found in [137] and [12]. Compared to the concentration mean, variance has shown to be sensitive on a spatial scale (averaging scale), hence the differences between numerically and analytically obtained concentration variance values shown in Figure Fig. 5.42, due to different spatial scales used. The comparison exhibits an overall good match and the values of variance are within the upper limit of 0.25 , except for the bimodality present in the analytical solution.

The bimodal behavior of variance in analytical model, as discussed in Section 5.1.1, is mostly influenced by the shape of the mean concentration (the chosen one-dimensional Gaussian distribution) and the source size. However that influence is reduced in the downstream direction due to more intense effect of 
the physical processes, mainly molecular diffusion. Hence, the variance obtained by analytical model slowly progresses to a unimodal shape. Such behavior in the near-field zone was thoroughly discussed by [124, 140, 4, 5, 137]. On the other hand, the resolution of numerical model introduced an additional smoothing over the grid size causing the variance to be unimodal from the beginning. Nevertheless, this verification showed that proposed analytical model could be used as a screening tool for determining the concentration moments.

\subsubsection{Comparison between salinity measurements, MOHID re- sults, and the analytical solution}

The different results obtained by analytical modeling, measurements and MOHID modeling are described above. Nevertheless, here they are compared to each other in the most accurate way possible [59].
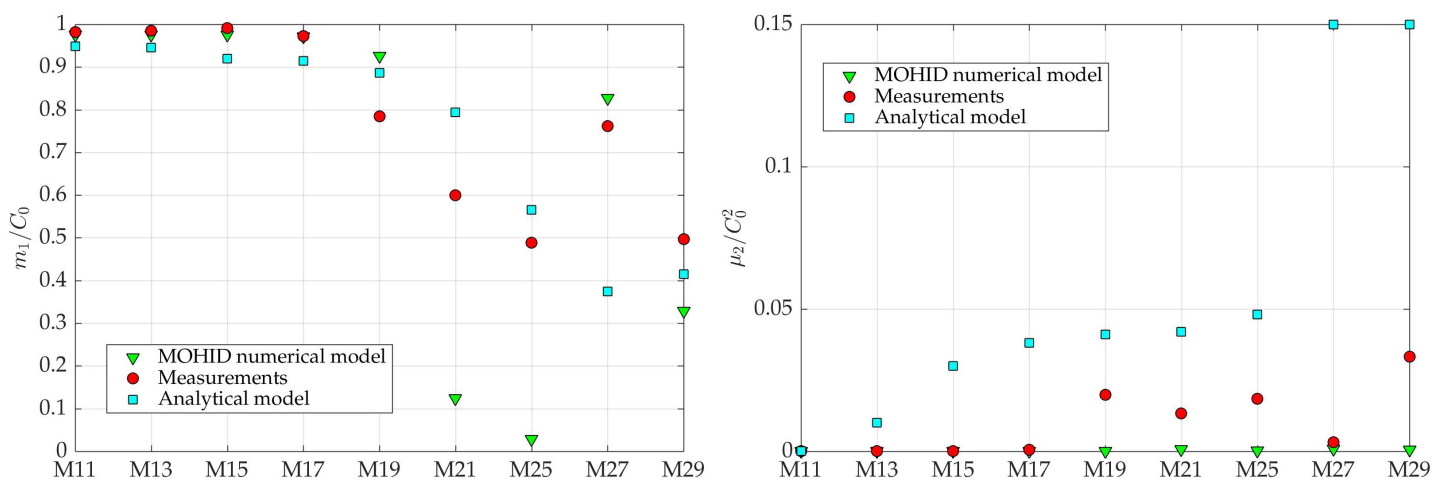

FIGURE 5.43: Comparison of the concentration mean and variance between analytical, measured and MOHID results at measurement points' positions.

Both the analytical and the MOHID model were set up using parameters from the field, including geometry, the initial velocity value, and additional data needed for MOHID (such as wind velocities, river flow setup, initial temperature and salinity input data, as explained in Section 4.3.2). The measurement data from the third campaign was used, since it provided the most data per point (approximately 300). The MOHID model simulated real conditions recorded at the day of measurements (December 29, 2015) and two previous days to allow for numerical stabilization. 

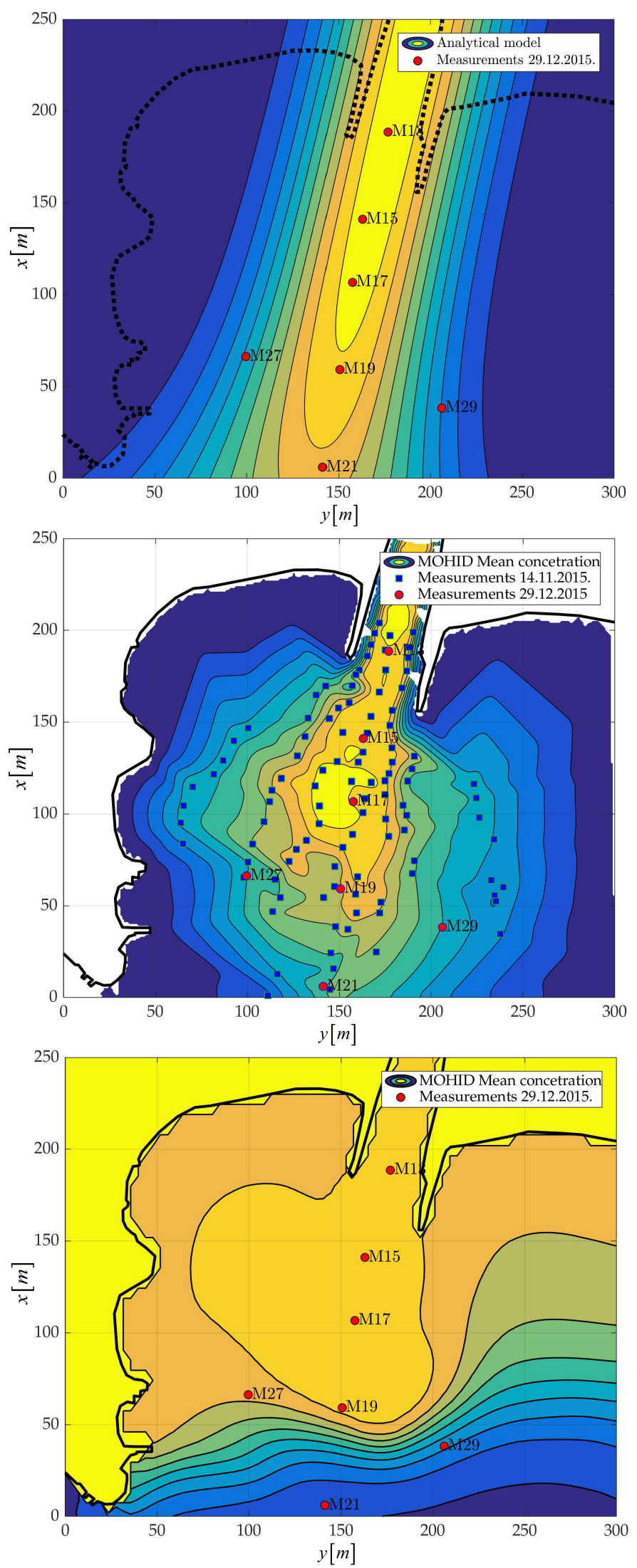

FIGURE 5.44: Comparison of the mean concentration field between the analytical model (up), proxy concentration measurements (middle), and the MOHID numerical model (down). 
Finally, it provided sets of salinity data (in the HDF file type) with a temporal resolution of $5 \mathrm{~s}$. Furthermore, only the data for eight hours of simulation was used, whereby those hours corresponded to the duration of the third measurement campaign including a few hours before and after the measurement. The salinity data was then transferred to Matlab to perform a transformation into proxy concentration by implementing the same formulation as for the measurements (Eq. (4.1)). Therefore, the comparisons between the concentration mean and variance at nine measurement points are given in Fig. 5.43.

The results are differently affected by the scale effect, as the analytical model is developed at the point, while both numerical and measured values are smoothed over the grid size ( $3.5 \mathrm{~m} \times 3.5 \mathrm{~m}$ for MOHID) and over the sampling volume (varying from 10 to $30 \mathrm{~m}^{2}$ ), respectively. This effect can easily be seen in Fig. 5.43, where the variance of the numerical model and measurements is much smaller than in the case of the analytically obtained one. However, concentration proxy moments (obtained from the measured salinity) showed more resemblance to the shape of analytical concentration moments. What the numerical model lacks in accuracy, it compensates with a larger spatial resolution, bigger volume of sampling points, and the ability to simulate different scenarios. Field work is limited due to the conditions and time required for taking reliable measurements and thus the numerical model, once thoroughly calibrated, might be used as a substitute for detailed field measurements. The initial input data should always be measured or obtained from official monitoring services $([111,102,103])$.

The cell size variability in the numerical model enables one to shift focus to a different scale of details. The qualitative comparison of the mean concentration field obtained by all three methods is given in Fig. 5.44. The measurement concentration field is obtained by combining quantitative data from the third campaign (with only nine measurement points, but 300 samples per point) with qualitative spatial data obtained in the second campaign (105 points, but only 20 samples per point). Nevertheless, the similarity between measurements and the analytical model is more emphasized as opposed to MOHID data. However, there are numerous possibilities within MOHID which might enable a better match. For instance, further research should include creating a denser mesh, especially in vertical discretization in order to better simulate salt wedge, whether that is provided by a nested model or simply a smaller global domain. There is also the potential usage of the Lagrangian module, where a more detailed parameter analysis should be implemented prior to its usage. 
For now, the provided comparisons have shown an acceptable level of matching for the analytical model derived in Chapter 2 to be used as a simple screening tool, and it is therefore implemented in an application in Chapter 6. Nevertheless, more ideas in the area of measurements and MOHID modeling are mentioned in Chapter 7.

\subsubsection{Potential limitation of the near-field approximation}

A strong mean velocity attenuation, as a result of different physical processes present in an estuary (e.g. flood, strong wind stress in upstream direction), may indicate the limitation of the near field assumption (Eq. (2.7)). That has been demonstrated by the non-physical behavior of the fourth concentration moment at large enough distances from the source (Fig. 5.9). In order to test this limiting distance, the behavior of the fourth concentration moment was defined as a function of the attenuation coefficient and outflow river velocity.

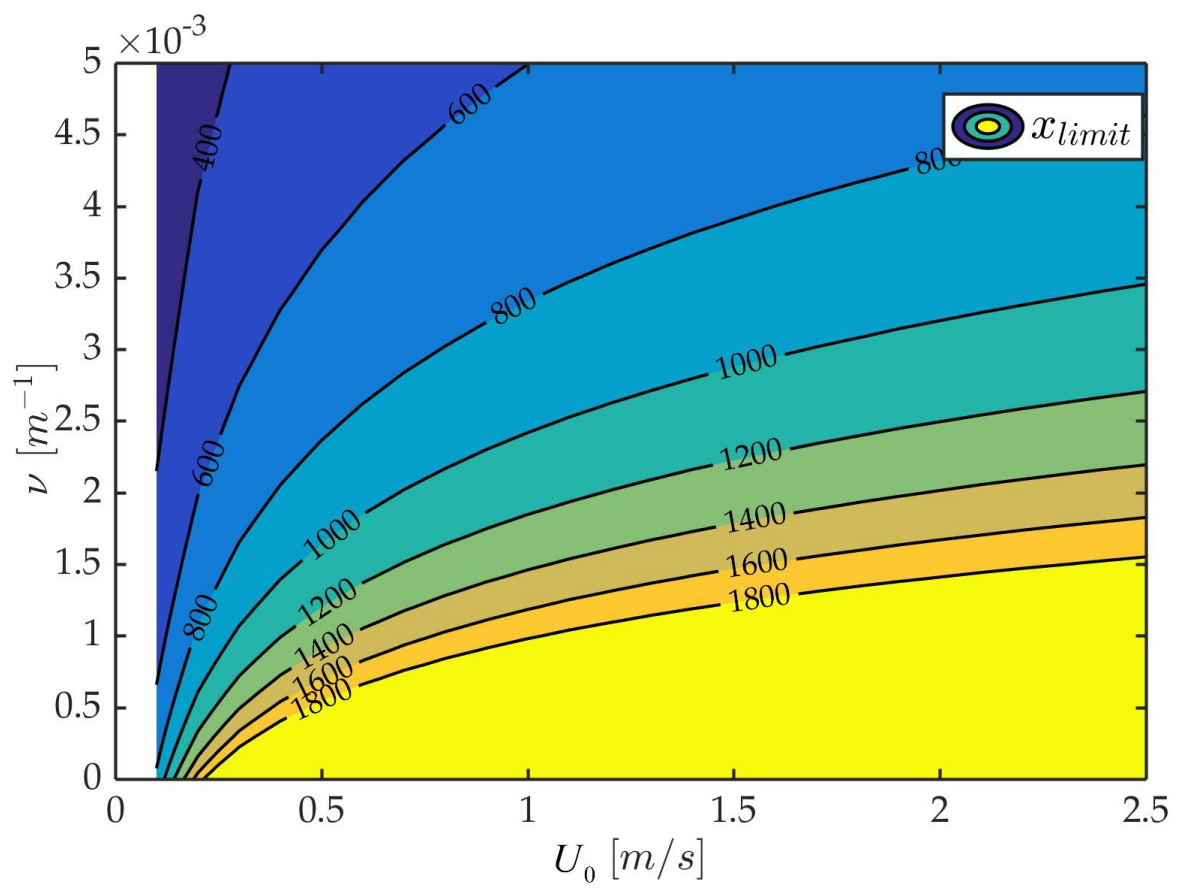

FIGURE 5.45: Limiting longitudinal distance from the river mouth as a function of outflow velocity and the attenuation coefficient as a potential definition of the limitation for the near field approximation.

Fig. 5.45 shows the limiting distance as being generally larger than $400 \mathrm{~m}$ even for a low outflow velocity and an attenuation coefficient up to $0.005 \mathrm{~m}^{-1}$. Hence, in most cases this limiting distance will even surpass $1000 \mathrm{~m}$, which is more than 
enough for most practical purposes, since other physical processes are expected to increase their impact after 1000 m anyway (e.g. sea currents).

\subsubsection{Comparison between beta distribution EMFs obtained by inversion of their measured and analytically defined mo- ments}

In the fourth measurement campaign the points formed two sections approximately perpendicular to the centerline (Fig. 4.10) at $93 \mathrm{~m}$ and $200 \mathrm{~m}$ from the river mouth. Using the Eq. (3.8), spatially integrated moments were obtained by integrating point statistics of proxy concentration data with approximately defined volumes as linearly distributed areas between measurement points at each section.
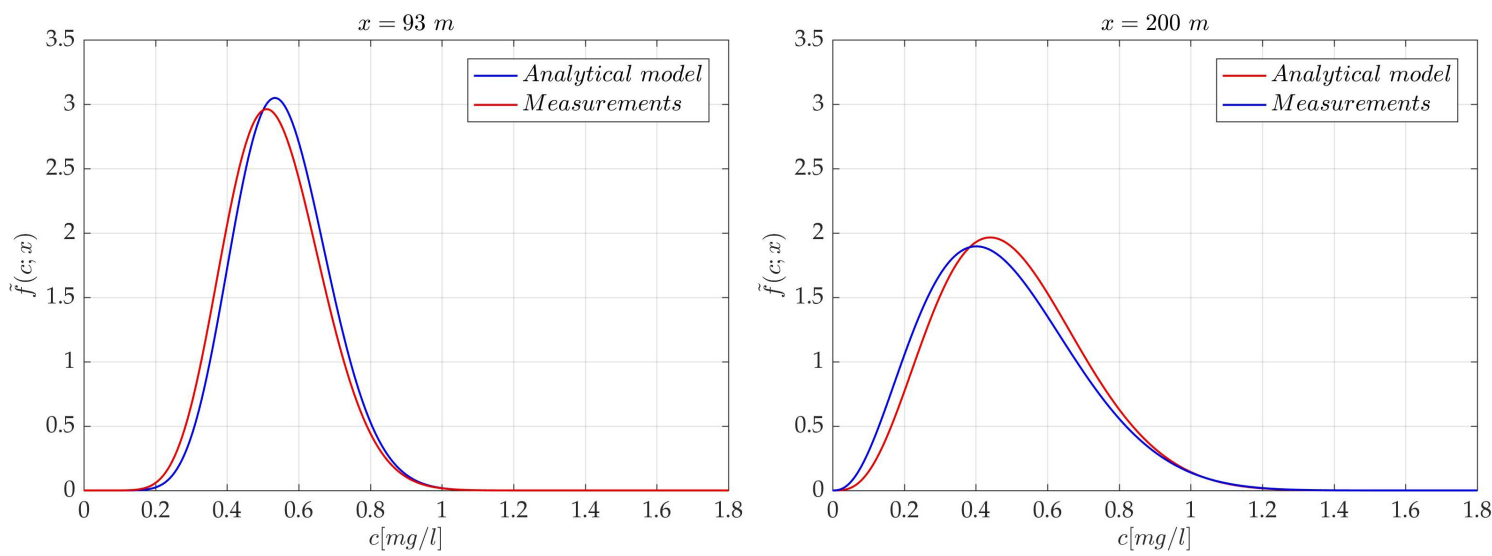

FIGURE 5.46: Comparison of analytical EMF and EMF obtained from proxy concentration measurements at $x=93 \mathrm{~m}$ (left) and $x=200 \mathrm{~m}$ (right) from the river mouth $\left(C_{0}=1 \mathrm{mg} / \mathrm{l}\right)$.

Since EMF moments are directly defined from those spatially integrated moments by Eq. (3.7), the first and second EMF moments were obtained for those sections. Although many more transversal points of salinity, i.e. proxy concentration data, would be preferable, these results indicate very good agreement on the expected mass fraction PDF. However, there are some ideas for upgrading the measurement procedure, specifically by applying simultaneous data acquisition at more points in an arbitrary direction (see Chapter 7). 


\subsubsection{The EMF concept as a potential contaminant dilution mea- sure in an estuary}

When presenting the EMF concept in Chapter 3, there is an idea of using it as a dilution measure in an estuary. This section attempts to deliver a practical application of the idea. Following the results presented in Fig. 5.18, the distances at which most of the dilution process has already been achieved $\left(F\left(c^{*} ; x\right)=0\right)$ are shown directly on the map of the Žrnovnica case study (Fig. 5.47).

It is, however, interesting to notice the differences in results when EMFs were obtained by direct integration of Beta distribution point PDFs versus EMFs obtained by inversion of their moments. That is particularly visible in the contaminant mass fraction exhibiting concentration above an arbitrary value $\left(c^{*}\right)$. In directly obtained $F\left(c^{*} ; x\right)$, presented in Fig. 5.14 , it is difficult to obtain results when the function approaches the zero, whereas the same function easily reaches zero when obtained from EMF moments (Fig. 5.18). The main reason for this behavior lies in numerical inaccuracies occurring when many Beta distribution PDFs are integrated over all concentration intervals for all points in a cross section. This issue increases with cross-sectional discretization, i.e. the more points at a section are used, the more inaccurate it becomes. Since those point PDFs tend to exhibit very low values as the distances from the source increase, it becomes inaccurate to calculate the EMFs by their integration. In conclusion, the EMFs obtained from their moments are a better solution that is more accurate and more quickly calculated.

This chapter has delivered many results obtained as part of the research for this thesis. It has covered different illustrative examples of analytical modeling, measurement results, and results obtained by numerical modeling. Some of the results showed good application and matching with measured or numerically modeled data (e.g. attenuation velocity model, the first two moments, EMF obtained by moment inversion, etc.), while others indicated limitations of the developed methodologies or verification procedures (part of MOHID and measurement results). Nevertheless, all of them have brought a lot of insight into the physical processes in this type of estuary and their potential descriptions, as well as posed new questions that might be investigated in the future (Chapter 7). 

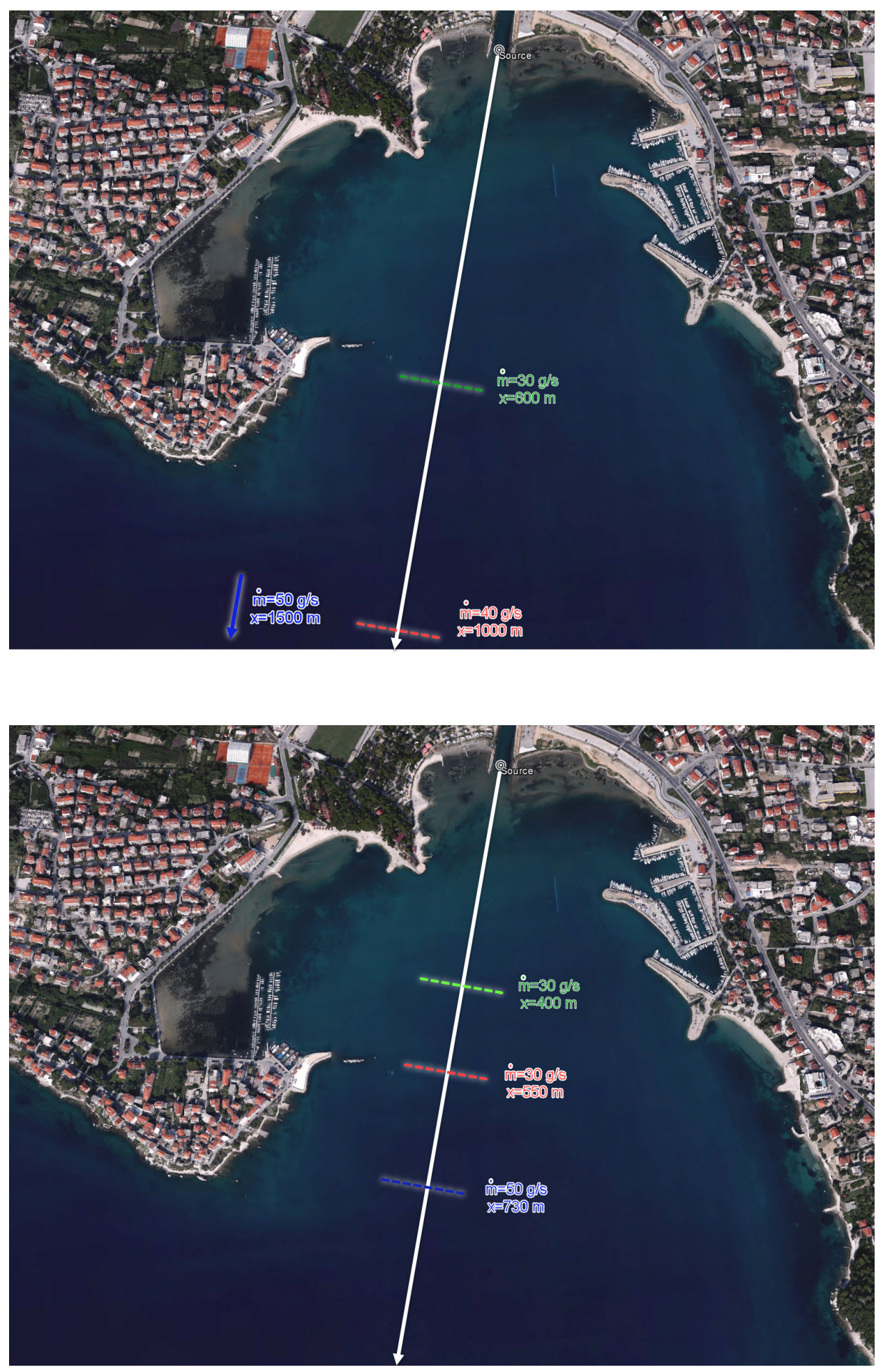

FIGURE 5.47: The seaward extension of the dilution process for $c^{*}=1 \mathrm{mg} / 1$ as a function of different estimated river loadings for constant mean velocity (up) and for a decreasing mean velocity with the attenuation factor of $\nu=0.002 \mathrm{~m}^{-1}$ (down). 



\section{Chapter 6}

\section{Practical application of the research conducted}

An attempt of utilizing the developed methodologies in a practical context has been made. This was enabled through a particular project which was implemented during the development of this thesis.

In 2014, the Croatian Ministry of Science and Education issued a call for project proposals in a form of research grants for young researchers within the Human Resources Development 2007-2013 Program. This program was fully funded by the European Social Fund and the Croatian State Budget. The main goal of the program was the professional development of young researchers and advancement of their competences by performing state-of-the-art research along with project management and increased international visibility. The project proposal was titled Risk assessment of the pollution caused by rivers and discharges in coastal areas (HR.3.2.01-0262) with an acronym CPoRT which stands for Coastal Pollution Risk Tool [36]. Since it was desirable to have a public domain product in the proposal, the CPoRT application was made as an implementation of the developed analytical methodologies into a user-friendly graphical interface, which was free to use. The proposal was selected for funding and the project was launched in the summer of 2015, lasting for 15 months and delivering many research results in this thesis. In the following several sections, a short overview of the project is outlined (Section 6.1), along with a description of the application (Section 6.2). Potential advantages of a more active collaboration with stakeholders are discussed in Section 6.3.

\subsection{Project synopsis}

The project was structured in six work packages, which were all connected to the project goals in some way (Fig. 6.1). 


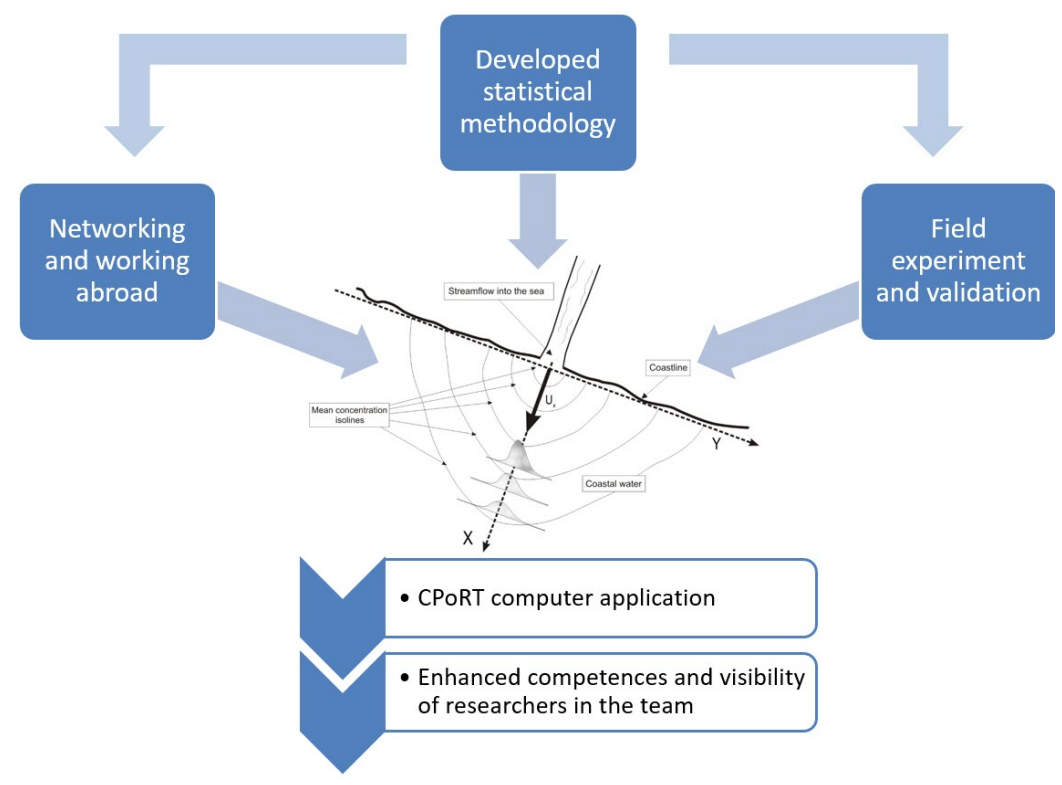

FIGURE 6.1: Schematic of project workflow.

Two of the working packages were strictly scientific and focused on the development of the analytical methodology covered by this thesis and the validation procedures (measurements and numerical modeling). The CPoRT application was one of the deliverables of these packages, along with the testing procedures.

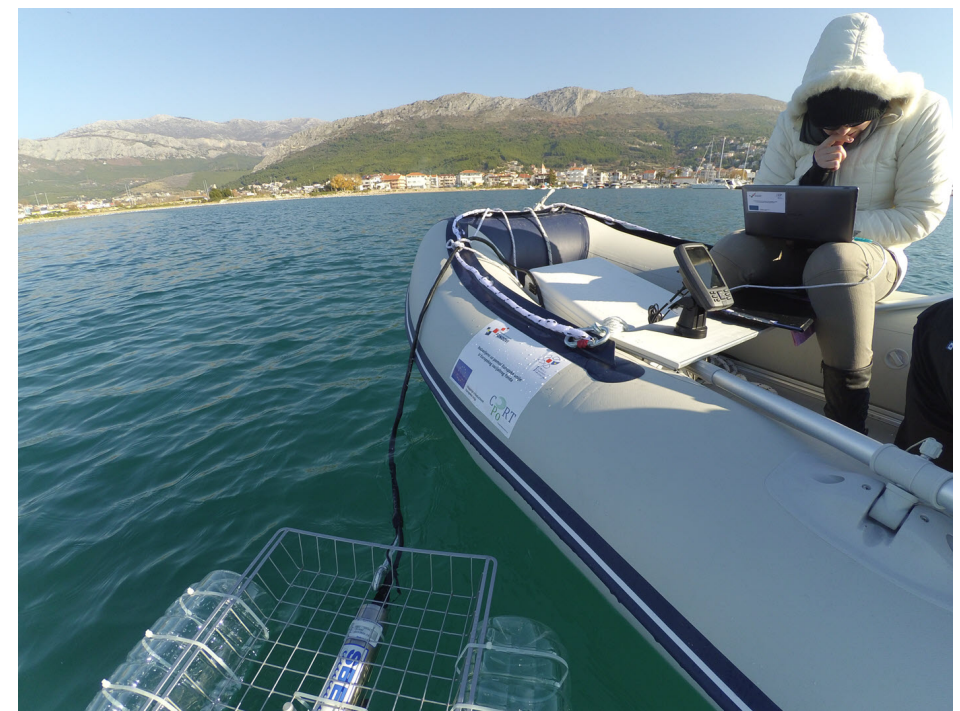

FIGURE 6.2: Field measurements using the procured equipment (with the mandatory project visibility items).

Measurement campaigns described in Section 4.2.2 were also part of the research packages. An inflatable boat with an outboard engine, a GPS device and a 
portable PC, procured by the project's funding, facilitated the measurement procedure (Fig. 6.2).

The third package included mobility and networking activities which enabled the CPoRT team (two researchers) to network with scientists from a similar area of research interest by conducting a part of the research in Lisbon, Portugal, at the Instituto Superior Técnico with the MARETEC research group (Section 4.3.2) and participating at the European Geosciences Union conference in 2016 ([59]). This package was very important for both the project and the thesis, since it enabled access to the MOHID numerical model. The fourth package mostly focused on knowledge transfer activities and the dissemination of research results. These activities were accomplished by publications while carrying out the project and afterwards $[58,59,56,55]$, by organizing workshops with stakeholders (more in Section 6.3), and launching an online forum for CPoRT users [35].

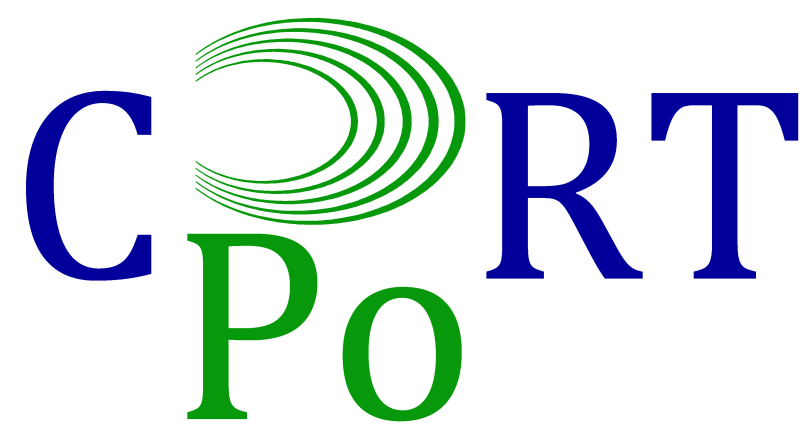

FIGURE 6.3: CPoRT project logo.

The fifth and sixth work packages were obligatory under the program and included standard activities related to the visibility of funding and project management. The visibility activities consisted of creating an official website for the project, designing the project logo (Fig. 6.3) and other official promotional materials (leaflets, posters), and finally the related media coverage.

Project management activities included coordination and monitoring of activities, procurement management, and writing reports every three months. The lead researcher was also the project manager and the author of this thesis, since the results of the project have greatly contributed to this thesis. Nevertheless, project activities were supervised by an expert in the research area (the thesis supervisor) and by an expert in project management. 


\subsection{CPoRT application}

As described in the preceding section, one of the main outcomes of the project was the computer application - a simple screening tool named CPoRT. The application has implemented the analytical methodology covered by Chapter 2 . The CPoRT application aims to raise awareness of the importance of preserving water resources with a thematic emphasis on coastal waters that are burdened by the pollution from rivers. Due to its simplicity, it is supposed to be user-friendly and available to different stakeholders.

The coding and the application was developed in the Matlab integrated development environment [100]. Fig. 6.4 shows the main working interface, along with an example of mean concentration visualization. The application has a fully graphical interface, implemented as a single main window and a series of secondary windows for the calculation and visualization of data. To allow for a better spatial reference, there is an option of uploading a map which may in fact be a screenshot taken from Google Earth or an image from any other freely available spatial data service. However, the scaling and rotation factors in the working interface need to be adjusted in order to ensure a good spatial fit.

In general, a very limited amount of input data is required, which makes CPoRT quick and practical. The data is organized in two blocks: parameters and domain. An arbitrary rectangular domain may be set according to a user's interest when analyzing an area, and it is defined by the initial and final values of $X$ or $Y$, and an increment. The parameters block requires more data, some of which is measured data, but when testing different scenarios, a user may obtain outcomes for different values. It is necessary to know the initial riverine velocity $[\mathrm{m} / \mathrm{s}]$ at the source, contaminant source concentration $\left[\mathrm{g} / \mathrm{m}^{3}\right]$, and the source width $[\mathrm{m}]$ representing the effective part of the river mouth width. The source concentration may be calculated in an additional dialog box if the contaminant mass flux is known ( $\dot{m}\left[\mathrm{~kg} /\right.$ day]). The molecular diffusion coefficient $\left(e_{m}\right)$ is set to $10^{-9} \mathrm{~m}^{2} / \mathrm{s}$ by default, which is a regular value for surface waters [117], even though it is user-editable. The turbulent diffusion coefficient $e_{t}\left[\mathrm{~m}^{2} / \mathrm{s}\right]$ should be assessed for the estuarine system in question. However, if no data is available, different values can be tested in order to assess its potential effect on the pollution plume spreading, especially for the worst-case scenario. Velocity attenuation may be set to a measured value or calculated within a dialog box if downstream centerline velocity data is known.

Fig. 6.5 provides an example of results obtained by the CPoRT application where the user selected three points of interest to obtain the probability of exceeding the 

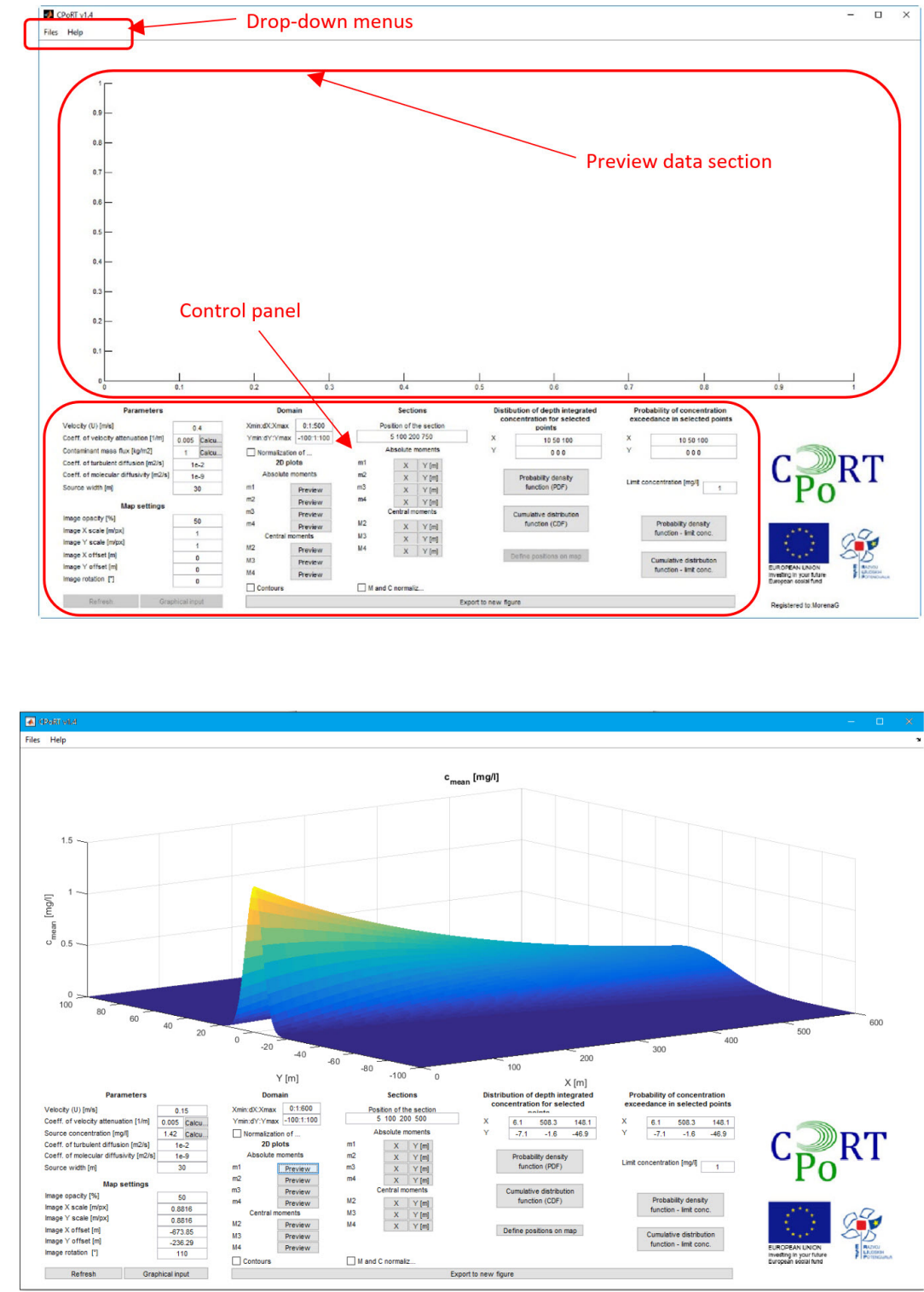

FIGURE 6.4: CPoRT application graphical interface in general (up) and an example of mean surface plot in CPoRT (down).

concentration limit ( $1 \mathrm{mg} / 1$ in this case). The plot is modified to emphasize the location of the case study and the positions of three chosen points where CDFs were calculated. Three points are chosen at different locations to describe the extents of the solution [56]. The first and the second point represent the longitudinal evolution of the expected concentration, while the third is set outside of the expected plume transversal spreading. 


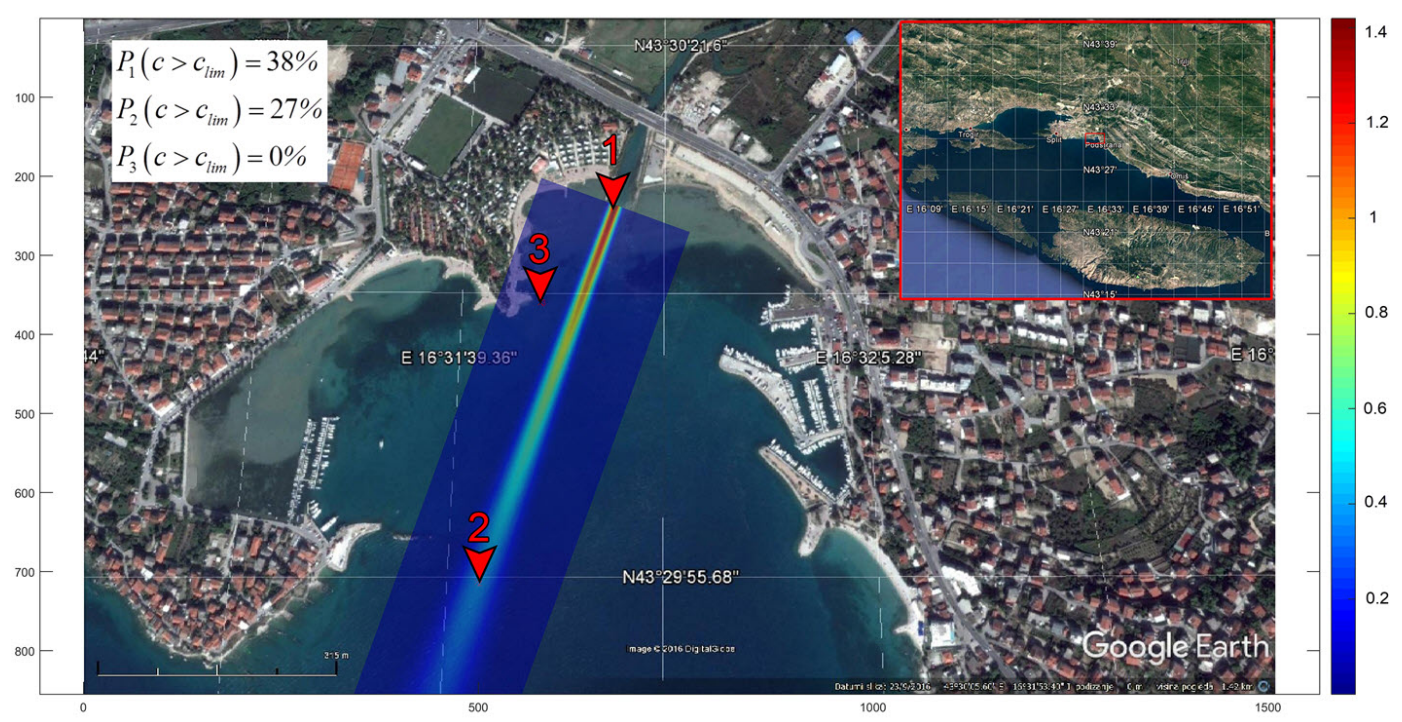

FIGURE 6.5: Illustrative results: the Žrnovnica estuary map with mean concentration results and the probability of exceeding the limit concentration $\left(c^{*}=1 \mathrm{mg} / \mathrm{l}\right)$ in chosen points.

The value of probability at the location of the third point is important when considering water quality at the beach of a local car campsite. Since the probability at the beach location is nearly zero, the area may be considered not threatened by the water quality reduction.

In conclusion, CPoRT implements a previously developed methodology for evaluation of concentration statistics for a conservative pollutant transported by a river into an estuary. It represents a simplified screening tool which can serve as a platform for raising awareness by grasping the idea of an estuarine ecosystem's resilience. Furthermore, it may be used to indicate potential hotspots which would require more detailed monitoring and/or complex numerical modeling. Its 2D solution is based on several approximations: near-field zone, steady and continuous river plume and conservative pollutant, and it lacks the details and calculation possibilities of common hydrodynamic numerical models. However, due to its simplicity, it generates results within seconds and preprocessing can be done in a few minutes. This simplicity represents an advantage in comparison to more complex models. CPoRT has its place as a tool for educational purposes, raising awareness, and helping stakeholders and decision makers to grasp a more general overview, while detailed analyses and integrated complex scenarios are still to be done by more sophisticated numerical models. Since CPoRT represents a simple tool for a decision support system, it may reveal additional potential when interfaced with the marine spatial data infrastructure [144] in its future development (see Section 7.2). 


\subsection{Stakeholder's involvement}

The stakeholder's involvement was part of the project package that involved knowledge transfer and dissemination activities. However, following some of the latest advancements toward public inclusion in the area of IWRM (Integrated Water Resources Management) as described by [15, 132, 73], or specifically for community-based ecosystem management [52], some ideas of participatory modeling have been attempted. Considering these aspects, the research problem covered by this thesis and the project, the CPoRT application finds its place within a broader framework (Fig. 6.6).

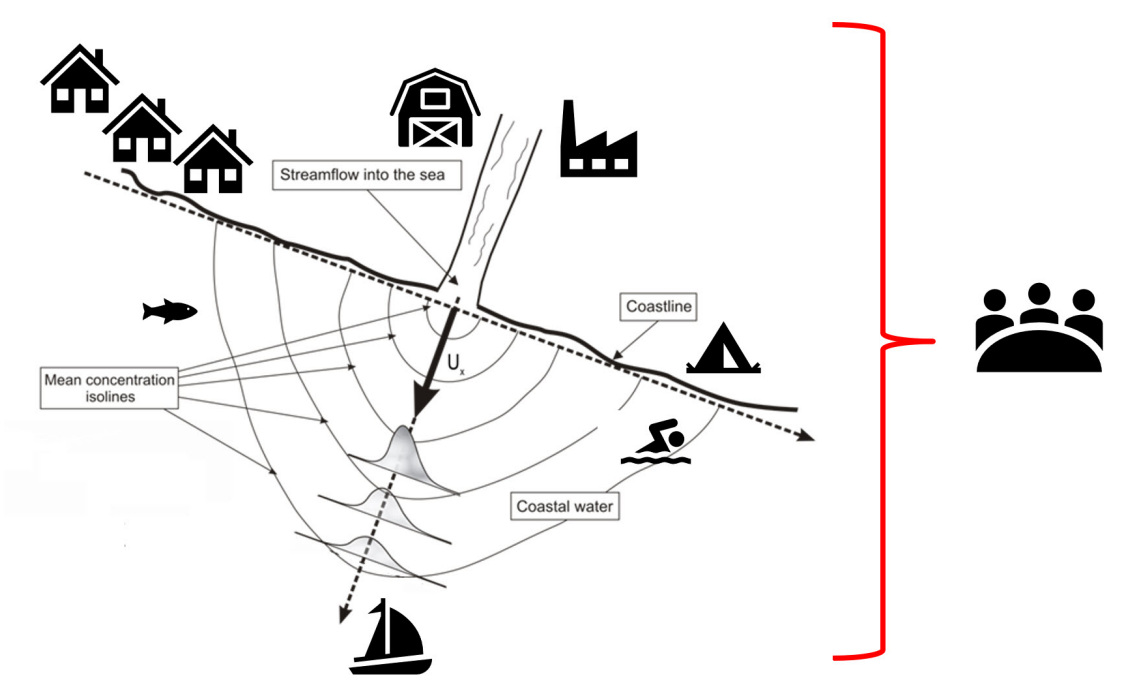

FIGURE 6.6: The research problem scheme within a broader framework.

In the early development stage (late spring of 2016), the use of the CPoRT application was tested on a group of 30 undergraduate civil engineering students at the University of Split. The initial assumption was that the participants had no significant knowledge of river-generated pollution in coastal seas. Nevertheless, the workshop was organized with the aim of gathering initial feedback, which would then be implemented in subsequent application development.

The second and more important test was the workshop (September 2016) which was part of project dissemination to which relevant stakeholders (i.e. end users of the screening tool) were invited. The group consisted of 44 participants from NGOs, universities (departments of science and technology, naval technology, institute for oceanography), SMEs, local water and sewerage companies, the banking sector, local government, and consulting agencies. The feedback from 

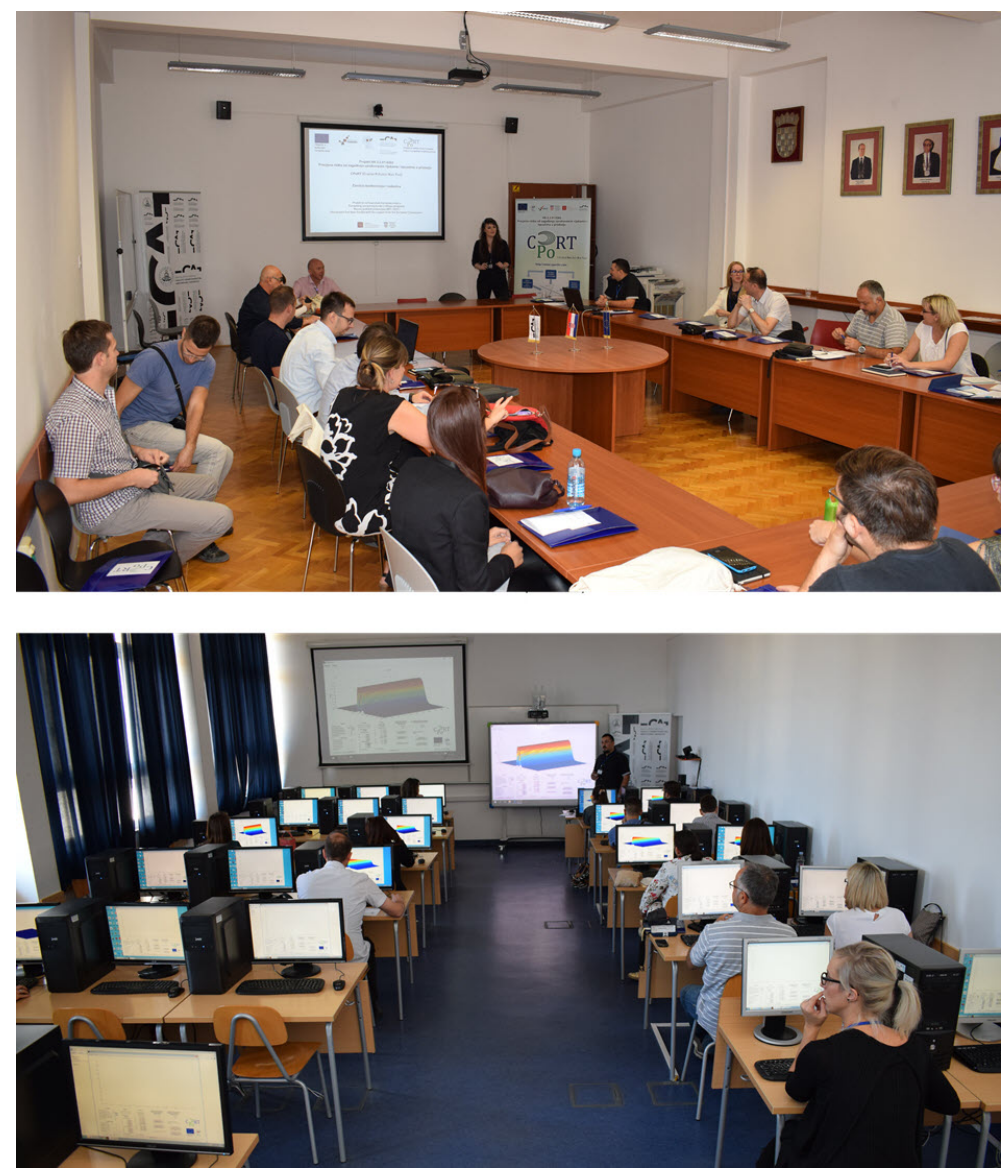

FIGURE 6.7: The stakeholders' workshop: presentation of project goals and CPoRT options (up) and a hands-on approach with potential users (down).

participants was received through both a written survey and simultaneous discussion during the workshop. The survey questions covered the topics of the participants' background knowledge, their effective daily usage of computers, the functionality and the design of the CPoRT application, and finally the effect of CPoRT on their understanding and potential decision-making in estuaries and coastal waters.

Both of the organized workshops delivered invaluable feedback regarding application functionality and its potential usefulness. For students, this was their first interaction with the issue of pollution in coastal zones. However, the survey results indicate that they happened to grasp the basic principles of the physical processes happening at the river-coastal sea interface in less than two hours. The students provided feedback regarding software functionality by detecting bugs and requesting a better explanation of the input parameters. In the stakeholders' workshop, most of the participants (30 out of 44) reported having achieved a better understanding of the issue. More than 50\% (25 out of 44 ) said that the 
application would be more user-friendly if it was web-based and GIS-integrated. Furthermore, all of them agreed that CPoRT may be fully or partially helpful in decision-making as a quick screening tool [56].

Finally, the key result of these activities is the idea of opening the door to collaborative modeling with stakeholders for future research directions and CPoRT versions. This approach [10] would facilitate a learning process through which stakeholders would inform the development and be part of the application coproduction, continuously in an interdependent communication and collaboration, in a joint effort for developing sustainable estuarine and coastal management. 



\section{Chapter 7}

\section{Conclusions and directions for future progress}

The following section outlines the most important outcomes of this thesis, along with newly revealed questions, which lead to future directions.

\subsection{Conclusions}

This thesis develops two analytical concepts for conservative solute transport in an estuary:

- Analytical recursive solution for concentration point PDF moments.

- Analytical recursive solution for the moments of the EMF function.

As mentioned in Chapter 2 and Chapter 3, the two concepts have already been applied, in a similar way, to other environmental flows (atmosphere and groundwater); however, in this case they are specifically applied to a river-dominated estuary and with an approximation of the near-field zone. The evaluated methodologies bring several key novelties:

- The developed point PDF moments are obtained by a recursive solution and do not depend on the Alpha-Beta formulation [26], unlike most similar research in other environmental flows [24, 140, 106]. This approach used a direct solution of the ADE written in terms of the concentration point moments Eq. (2.11) motivated by the work of [5].

- For the first time, the concentration point moments formulation used a nonuniform mean velocity (Eq. (2.17)) and delivered a recursive solution for that case (Eq. (2.21)). 
- Although spatially integrated statistics are a familiar concept ever since cloud average concentration statistics [25], there was no direct analytical solution for spatially integrated moments due to the problem of the "all space" volume definition. This thesis delivered an analytical recursive solution for spatially integrated moments and the EMF moments, since all space was defined as the volume of a cross-sectional profile (Chapter 3).

The above-specified points led to different applications in an estuary. For instance, due to the optimized and quick calculation of concentration point moments using the developed analytical solution, the concentration PDFs might be estimated at an arbitrary point in the estuary area. This was directly implemented in the CPoRT application as a screening tool available in the public domain [36]. On the other hand, the EMF concept developed for conservative solute transport in an estuary provided a potential dilution measure, which might also be used as a quick helping tool in decision support systems (see Fig. 5.18 and Fig. 5.47).

Furthermore, during the progression of ideas when validation procedures for the developed analytical models were investigated, two main concepts emerged. Due to similar dilution behavior of salinity attenuation in a stratified river-dominated estuary, the concept of using salinity as a concentration proxy was developed. Salinity was previously used as proxy for estimation of the dispersion coefficient in rivers and estuaries [78, 148]. However, this thesis brings a novelty by using salinity to obtain measured proxy concentration statistics, which resembles arbitrary conservative solute transport in an estuary.

Using a complex numerical model (MOHID) combined with salinity measurements has given an additional dimension to the validation procedure. It has also opened up many new ideas that might be investigated in further research (e.g. the influence of discretization, particularly vertical one, potential implementation of Lagrangian module and water quality module etc.).

Nevertheless, all developed methodologies have their limitations which have already been covered in the discussion section of Chapter 5. The near-field approximation or the zero molecular diffusion approximation becomes less applicable after relevant distance from the source (as covered by Section 5.4.4), but one might argue that most of the area of interest does fall in the near-field zone defined in this manner [105]. The application of developed analytical approach requires a river dominated and stratified estuaries since otherwise certain sea processes (e.g. tides, currents, sea upwelling...) become more significant. Moreover, the developed validation concepts of using combined salinity measurements and MOHID 
modeling should be further developed to achieve better quantitative comparison, since the obtained results are more in favor of qualitative matching. However, the EMF concept shows better matching since it is less sensitive to spatial reference. Finally, it is interesting to notice that almost all of the procedures and the research covered by this thesis has opened up more research questions and possibilities which are further discussed in future directions.

\subsection{Directions for future progress}

Key directions for future progress may be organized as follows:

1. Further development of the measurement procedure.

2. More extensive application of the MOHID numerical modeling system.

3. The implementation of the EMF concept in a versatile post-processing module which would enable its usage in different numerical models or in the analysis of measured data.

4. Creating a combined interface between the CPoRT and MSDI systems.

5. Including the key stakeholders in further model developments, hence engaging a more intense collaborative modeling process.

Ad 1.) One of the key findings during the development of this thesis is using salinity measurements for obtaining proxy concentration statistics. However, as mentioned in Section 5.4.5, it would be preferable to have more points at the same cross section, where salinity would be measured simultaneously. This approach would enable better comparison with analytical results and it would optimize the measurement procedure. The concept for this type of measurement is already being developed by the CPoRT team and is to be tested in the spring of 2018.

Ad 2.) Since there are numerous possibilities of using the MOHID modeling system, as described in Chapter 4, there are several directions where this research is aimed to continue afterwards. These directions include the implementation of a water quality module within MOHID, along with the setup for the Lagrangian module which would directly enable the simulation of different scenarios of pollution transport in estuaries or coastal waters in general, since a broader approach would be possible in that case. In the future work, the modeling in MOHID should be coupled with atmospheric model to obtain more realistic simulations with spatially and temporally variable atmospheric influences 
such as wind, pressures etc. By pairing up the EMF concept with this coupled ocean-atmosphere model, there are many possibilities to be achieved, especially if parallel processing is included. That approach would enable rather complex simulations to be executed on a cluster computing system, available at the University of Split.

Ad 3.) Following the idea of implementing the EMF concept in MOHID, a more versatile coding of the EMF module should be developed. The EMF concept should be a post-processing module which may be interfaced with any results of solute transport statistics whether they come from numerical modeling or from measurements. This module would be insensitive to the limitations defined in this thesis, since it would be evaluated directly from the data, instead of analytically. The development of both the EMF module and a new measurement procedure is an important part of a new research project of a broader scope, whose proposal is currently under review.

Ad 4.) The Marine Spatial Data Infrastructure is another thing yet to be developed in Croatia [144] and other similar EU countries in order to fulfill the guidelines of the INSPIRE Directive [33]. However, once such a service is put into operation, there is a lot of potential for developing the databases of estuaries and their characteristics along the Adriatic coast. This approach would engage monitoring services and, when connected to the CPoRT and EMF options, it would provide screening tools for multiple estuaries and coastal areas.

Ad 5.) And last but not least, the power of stakeholders' involvement cannot be emphasized enough, since their participation in an interactive learning process would enrich the modeling options and provide them with sustainable implementation of the developed methodologies. This direction would, however, serve in order to bridge the gap between the products of science and the work of policies and management by definition of collaborative learning [52]. Once active collaboration with stakeholders and the general public has been achieved, one may find that a slow but effective process of social changes has been launched. That type of social change is unavoidable in order to properly address the emerging challenges of climate changes, population growth, and different types of pressure requiring the implementation of sustainable management of all natural resources, which is quite emphasized in coastal zones. 


\section{Appendix A}

\section{ADE in terms of moments}

The fundamental ADE Eq. (1.1) is transformed in order to obtain the concentration mass balance in terms of standard moments $m_{n+1}$, starting with the following multiplication:

$$
\frac{\partial c(\mathbf{x}, t)}{\partial t}+\nabla \cdot[v(\mathbf{x}, t) c(\mathbf{x}, t)]=e_{m} \nabla^{2} c(\mathbf{x}, t) \mid \cdot c^{n}(n+1)
$$

Such equation is subsequently divided into three separate parts to enable a better representation of the conducted process:

$$
\underbrace{\frac{\partial c(\mathbf{x}, t)}{\partial t} \cdot c^{n}(n+1)}_{\text {part I.a }}+\underbrace{\nabla \cdot[v(\mathbf{x}, t) c(\mathbf{x}, t)] \cdot c^{n}(n+1)}_{\text {part I.b }}=\underbrace{e_{m} \nabla^{2} c(\mathbf{x}, t) \cdot c^{n}(n+1)}_{\text {part II. }}
$$

Part I.a describes why the function used to multiply the whole ADE is $f=c^{n}(n+$ 1):

$$
\begin{gathered}
\frac{\partial c}{\partial t} \cdot f=\frac{\partial c^{n+1}}{\partial t} \\
\frac{\partial c}{\partial t} \cdot f=(n+1) \cdot c^{n} \frac{\partial c}{\partial t} \\
f=c^{n}(n+1)
\end{gathered}
$$

And for part I.b:

$$
\begin{gathered}
\nabla(v c) \cdot c^{n}(n+1)=\nabla\left(v c^{n+1}\right) \\
(\nabla v \cdot c+v \cdot \nabla c) \cdot c^{n}(n+1)=\nabla v \cdot c^{n+1}+v(n+1) \cdot c^{n} \cdot \nabla c \\
\nabla v \cdot c^{n+1}(n+1)+v(n+1) \cdot c^{n} \cdot \nabla c=\nabla v \cdot c^{n+1}+v(n+1) \cdot c^{n} \cdot \nabla c
\end{gathered}
$$

where $\nabla v=0$ due to mass conservation in the observed turbulent flow of homogeneous incompressible fluid [85]. Part II. of the ADE is developed without the 
constant $e_{m}$ for the sake of simplicity:

$$
\begin{gathered}
\nabla^{2} c \cdot f=\nabla^{2}(c \cdot f)-c\left(\nabla^{2} f\right)-2(\nabla c)(\nabla f) \\
\nabla^{2} c \cdot c^{n}(n+1)=\nabla^{2}\left(c \cdot c^{n}(n+1)\right)-c\left(\nabla^{2} f\right)-2(\nabla c)(\nabla f) \\
\nabla^{2} c \cdot c^{n}(n+1)=(n+1)\left\{\nabla^{2} c^{n+1}-n\left[(n-1) c^{n-1}(\nabla c)^{2}+c^{n} \nabla^{2} c\right]-2(\nabla c) \cdot n \cdot c^{n-1}(\nabla c)\right\} \\
\nabla^{2} c \cdot c^{n}(n+1)=(n+1)\left\{\nabla^{2} c^{n+1}-n(n-1) c^{n-1}(\nabla c)^{2}-n c^{n} \nabla^{2} c-2 n c^{n-1}(\nabla c)^{2}\right\} \\
\frac{\nabla^{2} c \cdot c^{n}(n+1)=(n+1)\left\{\nabla^{2} c^{n+1}-n c^{n-1}(\nabla c)^{2}(n-1+2)-\underline{n \nabla^{2} c \cdot c^{n}}\right\}}{\nabla^{2} c \cdot c^{n}(n+1)(n+1)=(n+1)\left[\nabla^{2} c^{n+1}-n(n+1) c^{n-1}(\nabla c)^{2}\right]} \\
\nabla^{2} c \cdot c^{n}(n+1)=\nabla^{2} c^{n+1}-n(n+1) c^{n-1}(\nabla c)^{2}
\end{gathered}
$$

where derivatives of the chosen function $f=c^{n}(n+1)$ have been included:

$$
\begin{gathered}
\nabla f=n(n+1) c^{n-1} \nabla c \\
\nabla^{2} f=n(n+1)\left[(n-1) c^{n-2}(\nabla c)(\nabla c)+c^{n-1}(\nabla c)(\nabla c)\right]
\end{gathered}
$$

Finally, by connecting all parts (I.a+I.b=II.) of the above described transformations and by applying ensemble averaging, the initial ADE in the form of concentration has become ADE in the form of moments:

$$
\begin{gathered}
\frac{\partial c^{n+1}}{\partial t}+\nabla \cdot v c^{n+1}=e_{m} \nabla^{2} c^{n+1}-n(n+1) e_{m} c^{n-1}(\nabla c)^{2} \mid E \\
\frac{\partial \overline{c^{n+1}}}{\partial t}+\nabla \cdot \overline{v c^{n+1}}=e_{m} \nabla^{2} \overline{c^{n+1}}-n(n+1) e_{m} \overline{c^{n-1}(\nabla c)^{2}}
\end{gathered}
$$

which is then used in the rest of the analytical modeling starting from Eq. (2.4). 


\section{Appendix B}

\section{Detailed development of pollution concentration moments}

\section{B.1 The specific case of constant mean velocity}

Absolute moments $m_{n+1}$, following the Eq. (2.15) and central moments $\mu_{n+1}$ for the specific case of $\nu=0$, i.e. constant mean velocity, are derived for $n=$ $1,2,3,4,5$ and for the threshold concentration $c_{t}$. Moments are developed below. The second one with $n=1, k=2$ :

$$
\begin{aligned}
m_{2}(x) & =C_{0}^{n} \bar{c}(x)+\frac{2 \alpha}{U} e^{\frac{-2 \alpha}{U} x} \int_{0}^{x}\left[2 \bar{c}(\xi) c_{t}-c_{t}^{2}-C_{0} \bar{c}(\xi)\right] e^{\frac{2 \alpha}{U} \xi} d \xi \\
\mu_{2} & =m_{2}-m_{1}^{2} \\
\mu_{2}(x) & =\bar{c}(x)\left[C_{0}-\bar{c}(x)\right]+\frac{2 \alpha}{U} e^{\frac{-2 \alpha}{U} x} \int_{0}^{x}\left[2 \bar{c}(\xi) c_{t}-c_{t}^{2}-C_{0} \bar{c}(\xi)\right] e^{\frac{2 \alpha}{U} \xi} d \xi
\end{aligned}
$$

the third one with $n=2, k=6$ :

$$
\begin{aligned}
m_{3}(x) & =C_{0}^{2} \bar{c}(x)+\frac{6 \alpha}{U} e^{\frac{-6 \alpha}{U} x} \int_{0}^{x}\left[2 m_{2}(\xi) c_{t}-\bar{c}(\xi) c_{t}^{2}-C_{0}^{2} \bar{c}(\xi)\right] e^{\frac{6 \alpha}{U} \xi} d \xi \\
\mu_{3} & =m_{3}-3 m_{1} m_{2}+2 m_{1}^{3} \\
\mu_{3}(x) & =\bar{c}(x)\left[C_{0}-\bar{c}(x)\right]\left[C_{0}-2 \bar{c}(x)\right] \\
& +\frac{6 \alpha}{U} e^{\frac{-6 \alpha}{U} x} \int_{0}^{x}\left[2 m_{2}(\xi) c_{t}-\bar{c}(\xi) c_{t}^{2}-C_{0}^{2} \bar{c}(\xi)\right] e^{\frac{6 \alpha}{U} \xi} d \xi \\
& -\frac{6 \alpha}{U} \bar{c}(x) e^{\frac{-2 \alpha}{U} x} \int_{0}^{x}\left[2 \bar{c}(\xi) c_{t}-c_{t}^{2}-C_{0} \bar{c}(\xi)\right] e^{\frac{2 \alpha}{U} \xi} d \xi
\end{aligned}
$$


the fourth one with $n=3, k=12$ :

$$
\begin{aligned}
m_{4}(x) & =C_{0}^{3} \bar{c}(x)+\frac{12 \alpha}{U} e^{\frac{-12 \alpha}{U} x} \int_{0}^{x}\left[2 m_{3}(\xi) c_{t}-m_{2}(\xi) c_{t}^{2}-C_{0}^{3} \bar{c}(\xi)\right] e^{\frac{12 \alpha}{U} \xi} d \xi \\
\mu_{4} & =m_{4}-4 m_{3} m_{1}+6 m_{2} m_{1}^{2}-3 m_{1}^{4} \\
\mu_{4}(x) & =\bar{c}(x)\left[C_{0}-\bar{c}(x)\right]\left[C_{0}^{2}-3 C_{0} \bar{c}(x)+3 \bar{c}^{2}(x)\right] \\
& +\frac{12 \alpha}{U} e^{\frac{-12 \alpha}{U} x} \int_{0}^{x}\left[2 m_{3}(\xi) c_{t}-m_{2}(\xi) c_{t}^{2}-C_{0}^{3} \bar{c}(\xi)\right] e^{\frac{12 \alpha}{U} \xi} d \xi \\
& -\frac{24 \alpha}{U} \bar{c}(x) e^{\frac{-6 \alpha}{U} x} \int_{0}^{x}\left[2 m_{2}(\xi) c_{t}-\bar{c}(\xi) c_{t}^{2}-C_{0}^{2} \bar{c}(\xi)\right] e^{\frac{6 \alpha}{U} \xi} d \xi \\
& +\frac{12 \alpha}{U} \bar{c}^{2}(x) e^{\frac{-2 \alpha}{U} x} \int_{0}^{x}\left[2 \bar{c}(\xi) c_{t}-c_{t}^{2}-C_{0} \bar{c}(\xi)\right] e^{\frac{2 \alpha}{U} \xi} d \xi
\end{aligned}
$$

the fifth one with $n=4, k=20$ :

$$
\begin{aligned}
m_{5}(x) & =C_{0}^{4} \bar{c}(x)+\frac{20 \alpha}{U} e^{\frac{-20 \alpha}{U} x} \int_{0}^{x}\left[2 m_{4}(\xi) c_{t}-m_{3}(\xi) c_{t}^{2}-C_{0}^{4} \bar{c}(\xi)\right] e^{\frac{20 \alpha}{U} \xi} d \xi \\
\mu_{5} & =m_{5}-5 m_{4} m_{1}+10 m_{3} m_{1}^{2}-10 m_{3} m_{1}^{3}+4 m_{1}^{5} \\
\mu_{5}(x) & =\bar{c}(x)\left[C_{0}-\bar{c}(x)\right]\left[C_{0}^{3}-4 C_{0}^{2} \bar{c}(x)+6 C_{0} \bar{c}^{2}(x)-4 \bar{c}^{3}(x)\right] \\
& +\frac{20 \alpha}{U} e^{\frac{-20 \alpha}{U} x} \int_{0}^{x}\left[2 m_{4}(\xi) c_{t}-m_{3}(\xi) c_{t}^{2}-C_{0}^{4} \bar{c}(\xi)\right] e^{\frac{20 \alpha}{U} \xi} d \xi \\
& -\frac{60 \alpha}{U} \bar{c}(x) e^{\frac{-12 \alpha}{U} x} \int_{0}^{x}\left[2 m_{3}(\xi) c_{t}-m_{2}(\xi) c_{t}^{2}-C_{0}^{3} \bar{c}(\xi)\right] e^{\frac{12 \alpha}{U} \xi} d \xi \\
& +\frac{60 \alpha}{U} \bar{c}^{2}(x) e^{\frac{-6 \alpha}{U} x} \int_{0}^{x}\left[2 m_{2}(\xi) c_{t}-\bar{c}(\xi) c_{t}^{2}-C_{0}^{2} \bar{c}(\xi)\right] e^{\frac{6 \alpha}{U} \xi} d \xi \\
& -\frac{20 \alpha}{U} \bar{c}^{3}(x) e^{\frac{-2 \alpha}{U} x} \int_{0}^{x}\left[2 \bar{c}(\xi) c_{t}-c_{t}^{2}-C_{0} \bar{c}(\xi)\right] e^{\frac{2 \alpha}{U} \xi} d \xi
\end{aligned}
$$


the sixth one with $n=5, k=30$ :

$$
\begin{aligned}
m_{6}(x) & =C_{0}^{5} \bar{c}(x)+\frac{30 \alpha}{U} e^{\frac{-30 \alpha}{U} x} \int_{0}^{x}\left[2 m_{5}(\xi) c_{t}-m_{4}(\xi) c_{t}^{2}-C_{0}^{5} \bar{c}(\xi)\right] e^{\frac{30 \alpha}{U} \xi} d \xi \\
\mu_{6} & =m_{6}-6 m_{5} m_{1}+15 m_{4} m_{1}^{2}-20 m_{3} m_{1}^{3}+15 m_{2} m_{1}^{4}-5 m_{1}^{6} \\
\mu_{5}(x) & =\bar{c}(x)\left[C_{0}-\bar{c}(x)\right]\left[C_{0}^{4}-5 C_{0}^{3} \bar{c}(x)+10 C_{0}^{2} \bar{c}^{2}(x)-10 C_{0} \bar{c}^{3}(x)+5 \bar{c}^{4}(x)\right] \\
& +\frac{30 \alpha}{U} e^{\frac{-30 \alpha}{U} x} \int_{0}^{x}\left[2 m_{5}(\xi) c_{t}-m_{4}(\xi) c_{t}^{2}-C_{0}^{5} \bar{c}(\xi)\right] e^{\frac{30 \alpha}{U} \xi} d \xi \\
& -\frac{120 \alpha}{U} \bar{c}(x) e^{\frac{-20 \alpha}{U} x} \int_{0}^{x}\left[2 m_{4}(\xi) c_{t}-m_{3}(\xi) c_{t}^{2}-C_{0}^{4} \bar{c}(\xi)\right] e^{\frac{20 \alpha}{U} \xi} d \xi \\
& +\frac{180 \alpha}{U} \bar{c}^{2}(x) e^{\frac{-12 \alpha}{U} x} \int_{0}^{x}\left[2 m_{3}(\xi) c_{t}-m_{2}(\xi) c_{t}^{2}-C_{0}^{3} \bar{c}(\xi)\right] e^{\frac{12 \alpha}{U} \xi} d \xi \\
& -\frac{120 \alpha}{U} \bar{c}^{3}(x) e^{\frac{-6 \alpha}{U} x} \int_{0}^{x}\left[2 m_{2}(\xi) c_{t}-\bar{c}(\xi) c_{t}^{2}-C_{0}^{2} \bar{c}(\xi)\right] e^{\frac{6 \alpha}{U} \xi} d \xi \\
& +\frac{30 \alpha}{U} \bar{c}^{4}(x) e^{\frac{-2 \alpha}{U} x} \int_{0}^{x}\left[2 \bar{c}(\xi) c_{t}-c_{t}^{2}-C_{0} \bar{c}(\xi)\right] e^{\frac{2 \alpha}{U} \xi} d \xi
\end{aligned}
$$

\section{B.2 The case of non-uniform mean velocity}

When introducing the non-uniform mean velocity, absolute moments $m_{n+1}$ and central moments $\mu_{n+1}$ following the Eq. (2.22) are derived for $n=1,2,3,4,5$ and for the threshold concentration $c_{t}$. Depth integrated, mean velocity profile is given in:

$$
U(x)=U_{0} e^{-\nu x}
$$

When this kind of mean velocity profile is applied, due to kinematics ( $U=$ $d x / d t), t=t(x)$ is defined:

$$
t=\int_{0}^{x} \frac{d \xi}{U}=\int_{0}^{x} \frac{d \xi}{U_{0} e^{-\nu \xi}}=\int_{0}^{x} \frac{e^{\nu \xi}}{U_{0}} d \xi=\frac{1}{U_{0} \nu} e^{\nu \xi} \mid \begin{aligned}
& x \\
& 0
\end{aligned}=\frac{e^{\nu x}-1}{U_{0} \nu}
$$

thus, $x=\ln \left(U_{0} \nu t+1\right) / \nu$. 
In that way, for $\nu=0$, Eq. (B.17) transforms to $t=x / U_{0}$, which corresponds to the specific case of constant mean velocity. This is described in Eq. (B.18).

$$
t=\lim _{\nu \rightarrow 0} \frac{e^{\nu x}-1}{U_{0} \nu}=\left[\frac{0}{0}\right] \stackrel{L^{\prime} H}{\longrightarrow} \frac{\frac{d}{d \nu}\left(e^{\nu x}-1\right)}{\frac{d}{d \nu}\left(U_{0} \nu\right)}=\frac{x e^{0 \cdot x}}{U_{0}}=\frac{x}{U_{0}}
$$

Moments are further developed below.

The second one with $n=1, k=2$ :

$$
\begin{aligned}
m_{2}(x) & =C_{0} \bar{c}(x)+\frac{2 \alpha}{U_{0}} e^{\frac{-2 \alpha}{U_{0} \nu}\left(e^{\nu x}-1\right)} \\
& \times \int_{0}^{x}\left[2 \bar{c}(\xi) c_{t}-c_{t}^{2}-C_{0} \bar{c}(\xi)\right] e^{\left[\frac{2 \alpha}{U_{0} \nu}\left(e^{\nu \xi}-1\right)+\nu \xi\right]} d \xi \\
\mu_{2} & =m_{2}-m_{1}^{2} \\
\mu_{2}(x) & =\bar{c}(x)\left[C_{0}-\bar{c}(x)\right]+\frac{2 \alpha}{U_{0}} e^{\frac{-2 \alpha}{U_{0} \nu}\left(e^{\nu x}-1\right)} \\
& \times \int_{0}^{x}\left[2 \bar{c}(\xi) c_{t}-c_{t}^{2}-C_{0} \bar{c}(\xi)\right] e^{\left[\frac{2 \alpha}{U_{0} \nu}\left(e^{\nu \xi}-1\right)+\nu \xi\right]} d \xi
\end{aligned}
$$

the third one with $n=2, k=6$ :

$$
\begin{aligned}
m_{3}(x) & =C_{0}^{2} \bar{c}(x)+\frac{6 \alpha}{U_{0}} e^{\frac{-6 \alpha}{U_{0} \nu}\left(e^{\nu x}-1\right)} \\
& \times \int_{0}^{x}\left[2 m_{2}(\xi) c_{t}-\bar{c}(\xi) c_{t}^{2}-C_{0}^{2} \bar{c}(\xi)\right] e^{\left[\frac{6 \alpha}{U_{0} \nu}\left(e^{\nu \xi}-1\right)+\nu \xi\right]} d \xi \\
\mu_{3} & =m_{3}-3 m_{1} m_{2}+2 m_{1}^{3} \\
\mu_{3}(x) & =\bar{c}(x)\left[C_{0}-\bar{c}(x)\right]\left[C_{0}-2 \bar{c}(x)\right] \\
& +\frac{6 \alpha}{U_{0}} e^{\frac{-6 \alpha}{U_{0} \nu}\left(e^{\nu x}-1\right)} \int_{0}^{x}\left[2 m_{2}(\xi) c_{t}-\bar{c}(\xi) c_{t}^{2}-C_{0}^{2} \bar{c}(\xi)\right] e^{\left[\frac{6 \alpha}{U_{0} \nu}\left(e^{\nu \xi}-1\right)+\nu \xi\right]} d \xi \\
& -\frac{6 \alpha}{U_{0}} \bar{c}(x) e^{\frac{-2 \alpha}{U_{0} \nu}\left(e^{\nu x}-1\right)} \int_{0}^{x}\left[2 \bar{c}(\xi) c_{t}-c_{t}^{2}-C_{0} \bar{c}(\xi)\right] e^{\left[\frac{2 \alpha}{U_{0} \nu}\left(e^{\nu \xi}-1\right)+\nu \xi\right]} d \xi
\end{aligned}
$$


the fourth one with $n=3, k=12$ :

$$
\begin{aligned}
m_{4}(x) & =C_{0}^{3} \bar{c}(x)+\frac{12 \alpha}{U_{0}} e^{\frac{-12 \alpha}{U_{0} \nu}\left(e^{\nu x}-1\right)} \\
& \times \int_{0}^{x}\left[2 m_{3}(\xi) c_{t}-m_{2}(\xi) c_{t}^{2}-C_{0}^{3} \bar{c}(\xi)\right] e^{\left[\frac{12 \alpha}{U_{0} \nu}\left(e^{\nu \xi}-1\right)+\nu \xi\right]} d \xi \\
\mu_{4} & =m_{4}-4 m_{3} m_{1}+6 m_{2} m_{1}^{2}-3 m_{1}^{4} \\
\mu_{4} & =\bar{c}(x)\left[C_{0}-\bar{c}(x)\right]\left[C_{0}^{2}-3 C_{0} \bar{c}(x)+3 \bar{c}^{2}(x)\right] \\
& +\frac{12 \alpha}{U_{0}} e^{\frac{-12 \alpha}{U_{0} \nu}\left(e^{\nu x}-1\right)} \int_{0}^{x}\left[2 m_{3}(\xi) c_{t}-m_{2}(\xi) c_{t}^{2}-C_{0}^{3} \bar{c}(\xi)\right] e^{\left[\frac{12 \alpha}{U_{0} \nu}\left(e^{\nu \xi}-1\right)+\nu \xi\right]} d \xi \\
& -\frac{24 \alpha}{U_{0}} \bar{c}(x) e^{\frac{-6 \alpha}{U_{0} \nu}\left(e^{\nu x}-1\right)} \int_{0}^{x}\left[2 m_{2}(\xi) c_{t}-\bar{c}(\xi) c_{t}^{2}-C_{0}^{2} \bar{c}(\xi)\right] e^{\left[\frac{6 \alpha}{U_{0} \nu}\left(e^{\nu \xi}-1\right)+\nu \xi\right]} d \xi \\
& +\frac{12 \alpha}{U_{0}} \bar{c}^{2}(x) e^{\frac{-2 \alpha}{U_{0} \nu}\left(e^{\nu x}-1\right)} \int_{0}^{x}\left[2 \bar{c}(\xi) c_{t}-c_{t}^{2}-C_{0} \bar{c}(\xi)\right] e^{\left[\frac{2 \alpha}{U_{0} \nu}\left(e^{\nu \xi}-1\right)+\nu \xi\right]} d \xi
\end{aligned}
$$


the fifth one with $n=4, k=20$ :

$$
\begin{aligned}
m_{5}(x) & =C_{0}^{4} \bar{c}(x)+\frac{20 \alpha}{U_{0}} e^{\frac{-20 \alpha}{U_{0} e^{-\nu x}} x} \\
& \times \int_{0}^{x}\left[2 m_{4}(\xi) c_{t}-m_{3}(\xi) c_{t}^{2}-C_{0}^{4} \bar{c}(\xi)\right] e^{\left[\frac{20 \alpha}{U_{0} \nu}\left(e^{\nu \xi}-1\right)+\nu \xi\right]} d \xi \\
\mu_{5} & =m_{5}-5 m_{4} m_{1}+10 m_{3} m_{1}^{2}-10 m_{3} m_{1}^{3}+4 m_{1}^{5} \\
\mu_{5}(x) & =\bar{c}(x)\left[C_{0}-\bar{c}(x)\right]\left[C_{0}^{3}-4 C_{0}^{2} \bar{c}(x)+6 C_{0} \bar{c}^{2}(x)-4 \bar{c}^{3}(x)\right] \\
& +\frac{20 \alpha}{U_{0}} e^{\frac{-20 \alpha}{U_{0} \nu}\left(e^{\nu x}-1\right)} \int_{0}^{x}\left[2 m_{4}(\xi) c_{t}-m_{3}(\xi) c_{t}^{2}-C_{0}^{4} \bar{c}(\xi)\right] e^{\left[\frac{20 \alpha}{U_{0} \nu}\left(e^{\nu \xi}-1\right)+\nu \xi\right]} d \xi \\
& -\frac{60 \alpha}{U_{0}} \bar{c}(x) e^{\frac{-12 \alpha}{U_{0} \nu}\left(e^{\nu x}-1\right)} \int_{0}^{x}\left[2 m_{3}(\xi) c_{t}-m_{2}(\xi) c_{t}^{2}-C_{0}^{3} \bar{c}(\xi)\right] e^{\left[\frac{12 \alpha}{U_{0} \nu}\left(e^{\nu \xi}-1\right)+\nu \xi\right]} d \xi \\
& +\frac{60 \alpha}{U_{0}} \bar{c}^{2}(x) e^{\frac{-6 \alpha}{U_{0} \nu}\left(e^{\nu x}-1\right)} \int_{0}^{x}\left[2 m_{2}(\xi) c_{t}-\bar{c}(\xi) c_{t}^{2}-C_{0}^{2} \bar{c}(\xi)\right] e^{\left[\frac{6 \alpha}{U_{0} \nu}\left(e^{\nu \xi}-1\right)+\nu \xi\right]} d \xi \\
& -\frac{20 \alpha}{U_{0}} \bar{c}^{3}(x) e^{\frac{-2 \alpha}{U_{0} \nu}\left(e^{\nu x}-1\right)} \int_{0}^{x}\left[2 \bar{c}(\xi) c_{t}-c_{t}^{2}-C_{0} \bar{c}(\xi)\right] e^{\left[\frac{2 \alpha}{U_{0} \nu}\left(e^{\nu \xi}-1\right)+\nu \xi\right]} d \xi
\end{aligned}
$$


the sixth one with $n=5, k=30$ :

$$
\begin{aligned}
& m_{6}(x)=C_{0}^{5} \bar{c}(x)+\frac{30 \alpha}{U} e^{\frac{-30 \alpha}{U_{0}^{\nu}}\left(e^{\nu x}-1\right)} \\
& \times \int_{0}^{x}\left[2 m_{5}(\xi) c_{t}-m_{4}(\xi) c_{t}^{2}-C_{0}^{5} \bar{c}(\xi)\right] e^{\left[\frac{30 \alpha}{U_{0} \nu}\left(e^{\nu \xi}-1\right)+\nu \xi\right]} d \xi \\
& \mu_{6}=m_{6}-6 m_{5} m_{1}+15 m_{4} m_{1}^{2}-20 m_{3} m_{1}^{3}+15 m_{2} m_{1}^{4}-5 m_{1}^{6} \\
& \mu_{6}(x)=\bar{c}(x)\left[C_{0}-\bar{c}(x)\right]\left[C_{0}^{4}-5 C_{0}^{3} \bar{c}(x)+10 C_{0}^{2} \bar{c}^{2}(x)-10 C_{0} \bar{c}^{3}(x)+5 \bar{c}^{4}(x)\right] \\
& +\frac{30 \alpha}{U_{0}} e^{\frac{-30 \alpha}{U_{0} \nu}\left(e^{\nu x}-1\right)} \int_{0}^{x}\left[2 m_{5}(\xi) c_{t}-m_{4}(\xi) c_{t}^{2}-C_{0}^{5} \bar{c}(\xi)\right] e^{\left[\frac{30 \alpha}{U_{0} \nu}\left(e^{\nu \xi}-1\right)+\nu \xi\right]} d \xi \\
& -\frac{120 \alpha}{U_{0}} \bar{c}(x) e^{\frac{-20 \alpha}{U_{0} \nu}\left(e^{\nu x}-1\right)} \int_{0}^{x}\left[2 m_{4}(\xi) c_{t}-m_{3}(\xi) c_{t}^{2}-C_{0}^{4} \bar{c}(\xi)\right] e^{\left[\frac{20 \alpha}{U_{0} \nu}\left(e^{\nu \xi}-1\right)+\nu \xi\right]} d \xi \\
& +\frac{180 \alpha}{U_{0}} \bar{c}^{2}(x) e^{\frac{-12 \alpha}{U_{0} \nu}\left(e^{\nu x}-1\right)} \int_{0}^{x}\left[2 m_{3}(\xi) c_{t}-m_{2}(\xi) c_{t}^{2}-C_{0}^{3} \bar{c}(\xi)\right] e^{\left[\frac{12 \alpha}{U_{0} \nu}\left(e^{\nu \xi}-1\right)+\nu \xi\right]} d \xi \\
& -\frac{120 \alpha}{U_{0}} \bar{c}^{3}(x) e^{\frac{-6 \alpha}{U_{0} \nu}\left(e^{\nu x}-1\right)} \int_{0}^{x}\left[2 m_{2}(\xi) c_{t}-\bar{c}(\xi) c_{t}^{2}-C_{0}^{2} \bar{c}(\xi)\right] e^{\left[\frac{6 \alpha}{U_{0}^{\nu}}\left(e^{\nu \xi}-1\right)+\nu \xi\right]} d \xi \\
& +\frac{30 \alpha}{U_{0}} \bar{c}^{4}(x) e^{\frac{-2 \alpha}{U_{0} \nu}\left(e^{\nu x}-1\right)} \int_{0}^{x}\left[2 \bar{c}(\xi) c_{t}-c_{t}^{2}-C_{0} \bar{c}(\xi)\right] e^{\left[\frac{2 \alpha}{U_{0} \nu}\left(e^{\nu \xi}-1\right)+\nu \xi\right]} d \xi
\end{aligned}
$$

In conclusion, an arbitrary number of moments for both the uniform and the non-uniform case of the mean velocity field can be developed using Eq. (2.15) and Eq. (2.22). 



\section{Appendix C}

\section{Detailed development of spatially integrated moments}

\section{C.1 The specific case of constant mean velocity}

Spatially integrated moments $K_{n+1}$ for the specific case of $\nu=0$, i.e. constant mean velocity, are simply evaluated using a recursive solution (3.20) for any chosen $n$. Several examples are provided below.

For $n=1, k=2$ :

$$
K_{2}(x)=e^{-\frac{2 \alpha}{U} x}\left\{\frac{2 \alpha}{U} \int_{0}^{x}\left[2 K_{1}(\xi) c_{t}-K_{0}(\xi) c_{t}^{2}\right] e^{\frac{2 \alpha}{U} \xi} d \xi+K_{2}(0)\right\}
$$

For $n=2, k=6$ :

$$
K_{3}(x)=e^{-\frac{6 \alpha}{U} x}\left\{\frac{6 \alpha}{U} \int_{0}^{x}\left[2 K_{2}(\xi) c_{t}-K_{1}(x) c_{t}^{2}\right] e^{\frac{6 \alpha}{U} \xi} d \xi+K_{3}(0)\right\}
$$

For $n=3, k=12$ :

$$
K_{4}(x)=e^{-\frac{12 \alpha}{U} x}\left\{\frac{12 \alpha}{U} \int_{0}^{x}\left[2 K_{3}(\xi) c_{t}-K_{2}(\xi) c_{t}^{2}\right] e^{\frac{12 \alpha}{U} \xi} d \xi+K_{4}(0)\right\}
$$




\section{C.2 The case of non-uniform mean velocity}

Spatially integrated moments $K_{n+1}$ for the case of non-uniform mean velocity $\left(U(x)=U_{0} e^{-\nu x}\right)$ are simply evaluated using a recursive solution (3.22) for any chosen $n$. Several examples are provided below.

For $n=1, k=2$ :

$$
K_{2}(x)=e^{-\frac{2 \alpha}{U_{0} \nu}\left(e^{\nu x}-1\right)}\left\{\frac{2 \alpha}{U_{0}} \int_{0}^{x}\left[2 K_{1}(\xi) c_{t}-K_{0}(\xi) c_{t}^{2}\right] e^{\left[\frac{2 \alpha}{U_{0} \nu}\left(e^{\nu \xi}-1\right)+\nu \xi\right]} d \xi+K_{2}(0)\right\}
$$

For $n=2, k=6$ :

$$
K_{3}(x)=e^{-\frac{6 \alpha}{U_{0} \nu}\left(e^{\nu x}-1\right)}\left\{\frac{6 \alpha}{U_{0}} \int_{0}^{x}\left[2 K_{2}(\xi) c_{t}-K_{1}(\xi) c_{t}^{2}\right] e^{\left[\frac{6 \alpha}{U_{0} \nu}\left(e^{\nu \xi}-1\right)+\nu \xi\right]} d \xi+K_{3}(0)\right\}
$$

For $n=3, k=12$ :

$$
K_{4}(x)=e^{-\frac{12 \alpha}{U_{0} \nu}\left(e^{\nu x}-1\right)}\left\{\frac{12 \alpha}{U_{0}} \int_{0}^{x}\left[2 K_{3}(\xi) c_{t}-K_{2}(\xi) c_{t}^{2}\right] e^{\left[\frac{12 \alpha}{U_{0} \nu}\left(e^{\nu \xi}-1\right)+\nu \xi\right]} d \xi+K_{4}(0)\right\}
$$

\section{C.3 Spatially integrated moments in source}

Following the zero molecular diffusion approximation in the near-field zone (see Chapter 2), the absolute moments at source, are defined by:

$$
m_{n+1}(0)=C_{0}^{n}(0) m_{1}(0)
$$

In Chapter 3 it is shown that a one-dimensional Gaussian distribution is convenient for describing the mean concentration:

$$
\bar{c}(y, x)=\frac{L}{\sigma_{y} \sqrt{2 \pi}} e^{\frac{-y^{2}}{2 \sigma_{y}^{2}}}
$$


It is convenient to set $C_{0}=\bar{c}(0,0)$ as previously described by [139, 140], which can then be written in a Gaussian form. Therefore, by introducing Eq. (C.8) into Eq. (C.7), where $C_{0}(0)=L / \sigma_{y_{0}} \sqrt{2 \pi}$ and $m_{1}(0)=L / \sigma_{y_{0}} \sqrt{2 \pi} e^{-\frac{y^{2}}{2 \sigma_{y_{0}}}}$ the initial standard moments can be written as:

$$
m_{n+1}(0)=\left(\frac{L}{\sigma_{y_{0}} \sqrt{2 \pi}}\right)^{n+1} e^{-\frac{y^{2}}{2 \sigma_{y_{0}}^{2}}}
$$

As described in Chapter 3, the initial integrated moments are defined by:

$$
K_{n+1}(0)=\int_{V} m_{n+1}(0) d V=\int_{V}\left(\frac{L}{\sigma_{y_{0}} \sqrt{2 \pi}}\right)^{n+1} e^{-\frac{y^{2}}{2 \sigma_{y_{0}}^{2}}} d V
$$

which is then arranged into:

$$
K_{n+1}(0)=\left(\frac{L}{\sigma_{y_{0}} \sqrt{2 \pi}}\right)^{n+1} \int_{-\kappa \sigma_{y 0}}^{+\kappa \sigma_{y_{0}}} e^{-\frac{y^{2}}{2 \sigma_{y_{0}}^{2}}} d V
$$

where $\kappa$ describes the expected lateral spreading defining the available volume, e.g. $\kappa=3$. The integral on the right side is then solved by substitution and the erf function:

$$
\begin{gathered}
\sqrt{\pi} \frac{1}{\sqrt{\pi}} \int_{-t_{\lim }}^{+t_{\lim }} e^{-t^{2}} d t \sigma_{y_{0}} \sqrt{2}=\sqrt{\pi} \sigma_{y_{0}} \sqrt{2} \underbrace{\frac{1}{\sqrt{\pi}} \int_{-t_{\lim }}^{+t_{\lim }} e^{-t^{2}} d t}_{=\operatorname{erf}\left(t_{\lim }\right)}=\sigma_{y_{0}} \sqrt{2 \pi} \operatorname{erf}\left(\frac{\kappa}{\sqrt{2}}\right) \\
t=\frac{y}{\sigma_{y_{0}} \sqrt{2}} \rightarrow t_{\lim }=\frac{\kappa}{\sigma_{y_{0}} \sqrt{2}}=\frac{k}{\sqrt{2}} \\
d y=\sigma_{y_{0}} \sqrt{2} d t
\end{gathered}
$$

When such presentation is introduced back to Eq. (C.11), the final definition of $K_{n+1}(0)$ is:

$$
\begin{gathered}
K_{n+1}(0)=\left(\frac{L}{\sigma_{y_{0}} \sqrt{2 \pi}}\right)^{n+1} \sigma_{y_{0}} \sqrt{2 \pi} \operatorname{erf}\left(\frac{\kappa}{\sqrt{2}}\right) \\
K_{n+1}(0)=\frac{L^{n+1}}{\left(\sigma_{y_{0}} \sqrt{2 \pi}\right)^{n}} \operatorname{erf}\left(\frac{\kappa}{\sqrt{2}}\right)
\end{gathered}
$$





\section{Bibliography}

[1] A. Abulaban and J.L. Nieber. "Modeling the effects of nonlinear equilibrium sorption on the transport of solute plumes in saturated heterogeneous porous media". In: Advances in water resources 23.8 (2000), pp. 893905. DOI: $10.1016 /$ S0309-1708 (00)00010-5.

[2] Portuguese consulting company Action Modulers. Action Modulers. 2017. URL: http: / / www . actionmodulers.com/.

[3] Catherine M. Allen. "Numerical simulation of contaminant dispersion in estuary flows". In: Proceedings of the Royal Society of London A: Mathematical, Physical and Engineering Sciences. Vol. 381. 1780. The Royal Society. 1982, pp. 179-194.

[4] Roko Andricevic. "Effects of local dispersion and sampling volume on the evolution of concentration fluctuations in aquifers". In: Water Resources Research 34.5 (1998), pp. 1115-1129. DOI: 10.1029 / 98wr0 0260.

[5] Roko Andricevic. "Exposure concentration statistics in the subsurface transport". In: Advances in Water Resources 31.4 (2008), pp. 714-725. DOI: 10 . $1016 / j$.advwatres.2008.01.007.

[6] Roko Andricevic, Veljko Srzic, and Hrvoje Gotovac. "Risk characterization for toxic chemicals transported in aquifers". In: Advances in Water Resources 36.Supplement C (2012), pp. 86-97. DOI: 10 . 1016 / j . advwatres . 2011.04 .009$.

[7] Isabella Ascione Kenov et al. "Advances in Modeling of Water Quality in Estuaries". In: Remote Sensing and Modeling: Advances in Coastal and Marine Resources. Ed. by C. W. Finkl and C. Makowski. Springer International Publishing, 2014, pp. 237-276. DOI: 10 . 1007 / 978-3-319-063263_10.

[8] Group of Authors. General Ocean Turbulence Model, Community model. 2017. URL: http: //gotm.net/.

[9] K. Bandyopadhyay et al. "Maximum entropy and the problem of moments: A stable algorithm". In: Physical Review E 71.5 (2005), p. 057701. 
[10] Laura Basco-Carrera et al. "Collaborative modelling or participatory modelling? A framework for water resources management". In: Environmental Modelling E Software 91.Supplement C (2017), pp. 95-110. DOI: 10.1016 / j.envsoft.2017.01.014.

[11] George Keith Batchelor. "Diffusion in a file of homogeneous turbulence". In: Mathematical Proceedings of the Cambridge Philosophical Society 48.2 (1952), pp. 345-362. DOI: 10.1017 /S0305004100027687.

[12] Alberto Bellin and Yoram Rubin. "Eulerian-Lagrangian approach for modeling of flow and transport in heterogeneous geological formations". In: Wat. Resour. Res. 30.11 (1994), pp. 2913-2924. DOI: 10 . 1029/94WR01489.

[13] Alberto Bellin and Daniele Tonina. "Probability density function of nonreactive solute concentration in heterogeneous porous formations". In: Journal of Contaminant Hydrology 94.1-2 (2007), pp. 109-125. DOI: 10.1016 / j.jconhyd.2007.05.005.

[14] Adam L. Berger, Vincent J. Della Pietra, and Stephen A. Della Pietra. "A maximum entropy approach to natural language processing". In: Computational linguistics 22.1 (1996), pp. 39-71.

[15] Ilke Borowski and Matt Hare. "Exploring the Gap Between Water Managers and Researchers: Difficulties of Model-Based Tools to Support Practical Water Management". In: Water Resources Management 21.7 (July 2007), pp. 1049-1074. DOI: 10 .1007/s11269-006-9098-z.

[16] Kenneth A. Buch and Werner J.A. Dahm. "Experimental study of the finescale structure of conserved scalar mixing in turbulent shear flows. Part 2. Sc.1". In: J. Fluid Mech. 364 (1998), pp. 1-29. DOI: 10 . 1017 / S 0022112098008726.

[17] Fabio Bulleri and Maura G. Chapman. "The introduction of coastal infrastructure as a driver of change in marine environments". In: Journal of Applied Ecology 47.1 (2010), pp. 26-35. DOI: 10 . 1111 / j . 1365-2664 . $2009.01751 . x$.

[18] Hans Burchard, Karsten Bolding, and Manuel Ruiz Villarreal. “Three-dimensional modelling of estuarine turbidity maxima in a tidal estuary". In: Ocean Dynamics 54.2 (2004), pp. 250-265. DOI: 10.1007 /s10236-003-0073-4.

[19] Rose Campbell et al. "Modeling oil spill containment in coastal areas". In: International Oil Spill Conference Proceedings 2014.1 (2014), p. 299742. DOI: $10.7901 / 2169-3358-2014-1-299742.1$. 
[20] Vittorio M. Canuto et al. "Ocean turbulence. Part I: One-point closure model - Momentum and heat vertical diffusivities". In: Journal of Physical Oceanography 31.6 (2001), pp. 1413-1426. DOI: $10.1175 / 1520-0485$ (2001) $031<1413$ : OTP IOP $>2.0 . \mathrm{CO} ; 2$.

[21] Darren Carpenter. "A further demystification of the U-shaped probability distribution". In: Electromagnetic Compatibility, 2005. EMC 2005. 2005 International Symposium on. Vol. 2. IEEE. 2005, pp. 519-524.

[22] Hubert Chanson, Richard Brown, and Mark Trevethan. "Turbulence measurements in a small subtropical estuary under king tide conditions". In: Environmental Fluid Mechanics 12.3 (2012), pp. 265-289. DOI: 10 . 1007 / s10652-011-9234-z.

[23] P.C. Chatwin and C.M. Allen. "Mathematical Models of Dispersion in Rivers and Estuaries". In: Annual Review of Fluid Mechanics 17.1-608 (1985), pp. 119149. DOI: 10.1146/annurev.fluid.17.1.119.

[24] P.C. Chatwin, D.M. Lewis, and P.J. Sullivan. "Turbulent dispersion and the beta distribution". In: Environmetrics 6.4 (1995), pp. 395-402. DOI: 10 . 1002 /env. 3170060408 .

[25] P.C. Chatwin and P.J. Sullivan. "A simple and unifying physical interpretation of scalar fluctuation measurements from many turbulent shear flows". In: Journal of Fluid Mechanics 212 (1990), pp. 533-556. DOI: 10 . $1017 /$ S 0022112090002087.

[26] P.C. Chatwin and P.J. Sullivan. "Cloud-average concentration statistics". In: Mathematics and Computers in Simulation 32.1-2 (1990), pp. 49-57. DOI: $10.1016 / 0378-4754$ (90) 90214-4.

[27] P.C. Chatwin and P.J. Sullivan. "Measurements of concentration fluctuations in relative turbulent diffusion". In: Journal of Fluid Mechanics 94.1 (1979), pp. 83-101.

[28] P.C. Chatwin and P.J. Sullivan. "The intermittency factor of scalars in turbulence". In: Physics of Fluids A: Fluid Dynamics 1.1989 (1989), pp. 761-763. DOI: $10.1063 / 1.857372$.

[29] P.C. Chatwin and P.J. Sullivan. "The relative diffusion of a cloud of passive contaminant in incompressible turbulent flow". In: Journal of Fluid Mechanics 91.2 (1979), pp. 337-355. DOI: 10 . 1017/ S 0022112079000161.

[30] Arun Chawla et al. "Seasonal Variability and Estuary-Shelf Interactions in Circulation Dynamics of a River-dominated Estuary". In: Estuaries and Coasts 31.2 (2008), pp. 269-288. DOI: 10.1007 / s12237-007-9022-7. 
[31] R.T. Cheng, Shizuo Feng, and Pangen Xi. "On Lagrangian residual ellipse". In: Physics of Shallow Estuaries and Bays (1986), pp. 102-113.

[32] J.F. Cohen et al. "Estimates of coastal populations". In: Science 278 (1997), pp.1211-1212. DOI: 10.1126/science.278.5341.1209c.

[33] European Commission. INSPIRE Directive. 2017. URL: http: / / inspire. ec.europa.eu/.

[34] S.A. Condie and J.R. Andrewartha. "Circulation and connectivity on the Australian North West shelf". In: Continental Shelf Research 28.14 (2008), pp. 1724-1739.

[35] University of Split CPoRT team FCEAG. CPoRT online forum. 2017. URL: http://cporthr. freeforums.net/.

[36] University of Split CPoRT team FCEAG. CPoRT project official website. 2017. URL: http: / /www. cporthr. com.

[37] Douglas S. Crawford, Tony Saad, and Terry A. Ring. "Verification and validation of the maximum entropy method for reconstructing neutron flux, with MCNP5, Attila-7.1.0 and the GODIVA experiment". In: Annals of Nuclear Energy 53.Supplement C (2013), pp. 188-191. DOI: 10 . 1016 / j . anucene.2012.09.010.

[38] Amélie Darracq et al. “Quantification of advective solute travel times and mass transport through hydrological catchments". In: Environmental Fluid Mechanics 10.1-2 (2009), pp. 103-120. DOI: 10 . 1007 / s10652-0099147-2.

[39] F.P.J. De Barros et al. "A theoretical framework for modeling dilution enhancement of non-reactive solutes in heterogeneous porous media". In: Journal of Contaminant Hydrology 175-176 (2015), pp. 72-83. DOI: 10.1016 / j.jconhyd.2015.01.004.

[40] M.T. Delpey et al. "Effects of waves on coastal water dispersion in a small estuarine bay". In: Journal of Geophysical Research: Oceans 119.1 (2014), pp. 7086. DOI: $10.1002 / 2013 \mathrm{JC} 009466$.

[41] R.W. Derksen and P.J. Sullivan. "Moment Approximations for Probability Density Functions". In: Combustion and flame 81 (1990), pp. 378-391.

[42] Georgia Destouni et al. "Small unmonitored near-coastal catchment areas yielding large mass loading to the sea". In: Global Biogeochemical Cycles 22.4 (2008), n/a-n/a. DOI: $10.1029 / 2008$ GB003287. 
[43] Ricardo Deus et al. "Three-dimensional model for analysis of spatial and temporal patterns of phytoplankton in Tucuruí reservoir, Pará, Brazil". In: Ecological Modelling 253.Supplement C (2013), pp. 28-43. DOI: 10.1016 / j.ecolmodel.2012.10.013.

[44] K. Nadia Dimou and E. Eric Adams. "A Random-walk, Particle Tracking Model for Well-mixed Estuaries and Coastal Waters". In: Estuarine, Coastal and Shelf Science 37.1 (1993), pp. 99-110. DOI: 10 . 1006 / ecss . 1993 . 1044.

[45] James Donnell. "Surface fronts in estuaries: A review". In: Estuaries 16.1 (1993), pp. 12-39. DOI: 10 .2307/1352761.

[46] Community European. “Bathing Water Quality Directive 2006/7/EC". In: Official Journal of the European Union (OJ L 64) (2006). URL: http: / / eurlex.europa.eu/legal-content/EN/TXT/?uri=CELEX:32006L0007.

[47] Community European. "Marine Strategy Framework Directive 2008/56/EC". In: Official Journal of the European Union (OJ L 164) (2008). URL: http : / / eur-lex. europa . eu / legal-content / EN / TXT / ?uri=CELEX : $32000 \mathrm{~L} 0060$.

[48] Community European. “Water Framework Directive 2000/60/EC". In: Official Journal of the European Communities (OJ L 327) (2000). URL: http : / / eur-lex.europa.eu/legal-content/EN/TXT/ ?uri=celex: $32008 \mathrm{~L} 0056$.

[49] Architecture Faculty of Civil Engineering and Geodesy. Coastal Engineering Project for the Municipality of Podstrana. Tech. rep. University of Split, 2015.

[50] R Fernandes et al. "Combining operational models and data into a dynamic vessel risk assessment tool for coastal regions". In: Ocean Science 12.1 (2016), p. 285.

[51] Daniel Fernàndez-Garcia, Tissa H Illangasekare, and Harihar Rajaram. "Differences in the scale-dependence of dispersivity estimated from temporal and spatial moments in chemically and physically heterogeneous porous media". In: Advances in water resources 28.7 (2005), pp. 745-759.

[52] Christine Baumann Feurt. Collaborative learning guide for ecosystem management. 2008.

[53] Hugo B. Fischer et al. Mixing in inland and coastal waters. Academic Press, 1979 , p. 302. 
[54] Jerome Friedman, Trevor Hastie, and Robert Tibshirani. The elements of statistical learning. Vol. 1. Springer series in statistics New York, 2001.

[55] Morena Galesic, Roko Andricevic, and Vladimir Divic. "Integrated concentration statistics from river generated pollution in coastal zones". In: 4th Coastal and Maritime Mediterranean Conference. 2017.

[56] Morena Galesic, Roko Andricevic, and Vladimir Divic. "New screening tool for obtaining concentration statistics of pollution generated by rivers in estuaries". In: 15th International Conference on Environmental Science and Technology. 2017.

[57] Morena Galesic and Hrvoje Gotovac. "Potential impact of heterogeneity on groundwater age". In: Water Science E Technology: Water Supply 14.3 (2014), pp.398-404. DOI: 10.2166/ws .2013.212.

[58] Morena Galesic et al. "Concentration statistics of solute transport for the near field zone of an estuary". In: Advances in Water Resources 94 (2016), pp. 424-440. DOI: $10.1016 / j$.advwatres.2016.06.009.

[59] Morena Galesic et al. "Potential data used for validation of concentration statistics obtained using analytical model for conservative transport in an estuary". In: EGU General Assembly 2016. 2016.

[60] James N. Galloway and Ellis B. Cowling. "Reactive nitrogen and the world: 200 years of change". In: AMBIO: A Journal of the Human Environment 31.2 (2002), pp. 64-71.

[61] James N. Galloway et al. "Nitrogen cycles : past, present, and future". In: Biogeochemistry 70 (2004), pp. 153-226. DOI: 10 . 1007 / s10533-004$0370-0$.

[62] Neil K. Ganju et al. "Tidal and Groundwater Fluxes to a Shallow, Microtidal Estuary: Constraining Inputs Through Field Observations and Hydrodynamic Modeling". In: Estuaries and Coasts 35.5 (2012), pp. 1285-1298. DOI: $10.1007 / \mathrm{s} 12237-012-9515-x$.

[63] J. Ganoulis. Risk Analysis of Water Pollution. Wiley-VCH Verlag GmbH \& Co. KGaA, 2009.

[64] Erwan Garel and Óscar Ferreira. "Fortnightly Changes in Water Transport Direction Across the Mouth of a Narrow Estuary". In: Estuaries and Coasts 36.2 (2012), pp. 286-299. DOI: 10 . 1007 / s 12237-012-9566-z.

[65] Josette Garnier et al. "Transformations of nutrients (N, P, Si) in the turbidity maximum zone of the Seine estuary and export to the sea". In: Estuarine, Coastal and Shelf Science 90.3 (2010), pp. 129-141. 
[66] Moncho Gomez-Gesteira et al. "A two-dimensional particle tracking model for pollution dispersion in A Coruña and Vigo Rias (NW Spain)". In: Oceanologica Acta 22.2 (1999), pp. 167-177.

[67] Donald A Goolsby et al. Flux and sources of nutrients in the MississippiAtchafalaya river basin, Topic 3 Report for the integrated assessment of hypoxia in the Gulf of Mexico. Tech. rep. Silver Springs, Maryland: NOAA Coastal Ocean Program, 1999, p. 130.

[68] Hrvoje Gotovac, Vladimir Cvetkovic, and Roko Andricevic. "Flow and travel time statistics in highly heterogeneous porous media". In: Water Resources Research 45.7 (2009), n/a-n/a. DOI: 10 .1029/2008wr0 07168.

[69] Hrvoje Gotovac and Blaz Gotovac. "Maximum entropy algorithm with inexact upper entropy bound based on Fup basis functions with compact support”. In: Journal of computational physics 228.24 (2009), pp. 9079-9091.

[70] Marie Gren, Georgia Destouni, and Henrik Scharin. "Cost effective management of stochastic coastal water pollution". In: Environmental Modeling $\mathcal{E}$ Assessment 5.4 (2000), pp. 193-203.

[71] A Grimvall and P Stålnacke. "Riverine Inputs of Nutrients to the Baltic Sea". In: A Systems Analysis of the Baltic Sea. Ed. by Fredrik V Wulff, Lars A Rahm, and Per Larsson. Berlin, Heidelberg: Springer Berlin Heidelberg, 2001, pp. 113-131. DOI: 10 .1007/978-3-662-04453-7_4.

[72] Bruna Grizzeti and Fayçal Bouraoui. Assessment of Nitrogen and Phosphorus Environmental Pressure at European Scale. Tech. rep. Institute for Environment and Sustainability, 2006.

[73] Emeline Hassenforder, Alex Smajgl, and John Ward. “Towards understanding participatory processes: Framework, application and results". In: Journal of Environmental Management 157.Supplement C (2015), pp. 84-95. DOI: $10.1016 / j \cdot j e n v m a n .2015 .04 .012$.

[74] W.K. Heagy. "The Reduction Of Concentration Values In A Contaminant Cloud In Environmental Flows". PhD thesis. The University of Western Ontario, 1994.

[75] W.K. Heagy and P.J. Sullivan. "Cloud concentration statistics and dosage". In: Mathematical and computer modelling 21.9 (1995), pp. 61-65.

[76] W.K. Heagy and P.J. Sullivan. "The expected mass fraction". In: Atmospheric Environment 30.1 (1996), pp.35-47. DOI: $10.1016 / 1352-2310$ (95) $00282-4$. 
[77] A.W. Heemink. "Stochastic modelling of dispersion in shallow water". In: Stochastic Hydrology and hydraulics 4.2 (1990), pp. 161-174.

[78] David T. Ho, Peter Schlosser, and Theodore Caplow. "Determination of Longitudinal Dispersion Coefficient and Net Advection in the Tidal Hudson River with a Large-Scale, High Resolution SF6 Tracer Release Experiment". In: Environmental Science E Technology 36.15 (2002), pp. 3234-3241. DOI: 10.1021 /es015814+.

[79] R.W. Howarth et al. "Riverine Inputs of Nitrogen to the North Atlantic Ocean : Fluxes and Human Influences". In: Biogeochemistry 35.May 2016 (1996), pp. 75-139.

[80] A.T. Ippen and P.S. Eagleson. Estuary and coastline hydrodynamics. Engineering societies monographs. McGraw-Hill Book Co., 1966.

[81] J. Ivar do Sul and M.F. Costa. "Plastic pollution risks in an estuarine conservation unit". In: Proceedings 12th International Coastal Symposium (Plymouth, England), Journal of Coastal Research (2013). 2013, pp. 48-53. DOI: 10.2112 /SI65-009.1.

[82] Kenneth E. Iverson. "A programming language". In: Proceedings of the May 1-3, 1962, spring joint computer conference. ACM. 1962, pp. 345-351.

[83] Ewa Jasińska. "Motion of Salt Water and Associated Fronts in Tideless Estuaries". In: Estuaries 16.1 (1993), pp. 53-67. DOI: $10.2307 / 1352763$.

[84] Edwin T. Jaynes. "Information theory and statistical mechanics". In: Physical review 106.4 (1957), p. 620.

[85] W. Kollmann and J. Janicka. "The probability density function of a passive scalar in turbulent shear flows". In: The Physics of Fluids 25.10 (1982), pp. 1755-1769. DOI: $10.1063 / 1.863653$.

[86] Nino Krvavica et al. "Hydraulics of Stratified Two-layer Flow in Rječina Estuary". In: Landslides and flood hazard assessment. Ed. by Snježana M Arbanas and Željko Arbanas. Zagreb, 2013, pp. 1-5.

[87] Anju A. Kumar, S. Dipu, and V. Sobha. "Seasonal variation of heavy metals in cochin estuary and adjoining Periyar and Muvattupuzha rivers, Kerala, India". In: Global Journal of Environmental Research 5.1 (2011), pp. 15-20.

[88] Brian Edward Launder and Dudley Brian Spalding. "The numerical computation of turbulent flows". In: Computer methods in applied mechanics and engineering 3.2 (1974), pp. 269-289. DOI: 10 . $1016 / 0045-7825$ (74) 90029-2. 
[89] Daniel A. Lemley et al. "Towards the classification of eutrophic condition in estuaries". In: Estuarine, Coastal and Shelf Science 164 (2015), pp. 221-232. DOI: $10.1016 / j$.ecss.2015.07.033.

[90] Georg A. Lindgren, Georgia Destouni, and Amélie Darracq. “Inland Subsurface Water System Role for Coastal Nitrogen Load Dynamics and Abatement Responses". In: Environmental Science E Technology 41.7 (Apr. 2007), pp. 2159-2164. DOI: 10.1021 /es 062535 j.

[91] Jelena Loborec et al. "Analiza opasnosti od onečišćenja podzemnih voda u kršu na primjeru sliva izvora Jadro i Žrnovnica". In: Journal of the Croatian Association of Civil Engineers 67.11 (2015), pp. 1093-1103. DOI: 10 . $14256 /$ JCE. 1250.2015.

[92] Xiangcui Lv et al. "Random walk method for modeling water exchange: An application to coastal zone environmental management". In: Journal of Hydro-environment Research 13.Supplement C (2016), pp. 66-75. DOI: 10 . $1016 / j \cdot j$ her.2015.07.001.

[93] Florent Lyard et al. "Modelling the global ocean tides: modern insights from FES2004". In: Ocean Dynamics 56.5-6 (2006), pp. 394-415.

[94] Daniele L. Marchisio et al. "Quadrature method of moments for populationbalance equations". In: AIChE Journal 49.5 (2003), pp. 1266-1276.

[95] Mateus Marcos and Ramiro J.J. Neves, eds. Ocean modelling for coastal management - Case studies with MOHID. Vol. 68. C. IST Press, 2013, pp. 88-105. DOI: $10.1016 /$ j.ocemod.2009.02.009.

[96] Instituto Superior Técnico MARETEC. MARETEC Official website. 2017. URL: http: / / www . maretec . org.

[97] Instituto Superior Técnico MARETEC. MOHID Official website. 2017. URL: http: //www.mohid.com/.

[98] Instituto Superior Técnico MARETEC. MOHID Wiki. 2017. URL: http: //wiki.mohid.com.

[99] J. Margeta and A. Barić. "Rational approach to the solution of the problem of wastewaters management - the Kaštela Bay case". In: Global Nest: the International Journal 3.3 (2001), pp. 117-130.

[100] MATLAB. version 9.10.0 (R2016b). Natick, Massachusetts: The MathWorks Inc., 2016. 
[101] Lawrence R Mead and Nikos Papanicolaou. "Maximum entropy in the problem of moments". In: Journal of Mathematical Physics 25.8 (1984), pp. 24042417.

[102] Croatian Meteorological and Hydrological Service. Hydrological sector. 2017. URL: http://161.53.81.21/.

[103] Croatian Meteorological and Hydrological Service. Meteorological sector. 2017. URL: http://meteo.hr/index_en.php.

[104] Ebtessam Mohamed et al. "Application of a Mathematical Model for Estimating the Pattern of Pollution Dispersion at the Coast of Alexandria". In: Journal of King Abdulaziz University-Marine Sciences 18.1 (2007), pp. 149167. DOI: $10.4197 / \operatorname{mar} .18-1.9$.

[105] N. Mole. "An idealised model of turbulent dispersion: Two and three spatial dimensions". In: Environmetrics 21.November 2009 (2010), pp. 767-788. DOI: 10.1002 /env.1037.

[106] N. Mole and E.D. Clarke. "Relationships between higher moments of concentration and of dose in turbulent dispersion". In: Boundary-Layer Meteorology 73.1989 (1995), pp. 35-52. DOI: 10.1007 /BF 00708929.

[107] D.J. Moseley. "A closure hypothesis for contaminant fluctuations in turbulent flow". MA thesis. University of Westerm Ontario, 1991.

[108] NASA. Rio de la Plata. 2003. URL: https : / / earthobservatory . nasa. gov/IOTD/view.php?id=4028.

[109] Ramiro J.J. Neves. "Biodimensional model for residual circulation in coastal zones: application to the Sado Estuary". In: Annales geophysicae. Vol. 3. 4. Gauthier-Villars. 1985, pp. 465-471.

[110] P.S. Niraj, R. Balaji, and P. Vethamony. "Pre-monsoon hydrodynamic modeling of Goa coast, West coast of India". In: ISH Journal of Hydraulic Engineering 21.3 (2015), pp. 276-286.

[111] Institute of Oceanography and Fisheries. On-line data. 2017. URL: http: //www.izor.hr/.

[112] Emin Ozsoy and Umit Unluata. "Ebb-tidal flow characteristics near inlets". In: Estuarine, Coastal and Shelf Science 14.3 (1982), 251-IN3. DOI: 10 . $1016 / \mathrm{s} 0302-3524$ (82) $80015-7$.

[113] A.G. Punt, G.E. Millward, and J.R. Harris. "Modelling solute transport in the Tweed Estuary, UK using ECoS". In: Science of the Total Environment 314-316 (2003), pp. 715-725. DOI: $10.1016 /$ s $0048-9697$ (03) 00079-2. 
[114] Nancy N. Rabalais. "Nitrogen in aquatic ecosystems.” In: Ambio 31.2 (2002), pp. 102-112. DOI: $10.2307 / 4315222$.

[115] A. Rodriguez et al. "Pollutant dispersion in the nearshore region: Modelling and measurements". In: Water Science and Technology 32.9-10 (1995), pp. 169-178. DOI: $10.1016 / 0273-1223$ (96) 00088-1.

[116] M. Ruiz-Villarreal et al. "Hydrodynamic Model Study of the Ria de Pontevedra Under Estuarine Conditions". In: Estuarine, Coastal and Shelf Science 54.1 (2002), pp. 101-113. ISSN: 0272-7714. DOI: 10 . $1006 /$ ecss . 2001.0825.

[117] J.C. Rutherford. River Mixing. Chichester: John Wiley \& Sons Ltd., 1994. ISBN: 0-471-94282-0.

[118] Peter Salamon, Daniel Fernàndez-Garcia, and J. Jaime Gómez-Hernández. "A review and numerical assessment of the random walk particle tracking method". In: Journal of Contaminant Hydrology 87.3 (2006), pp. 277-305. DOI: $10.1016 / j \cdot j c o n h y d .2006 .05 .005$.

[119] Sofia Saraiva et al. "Modelling the influence of nutrient loads on Portuguese estuaries". In: Hydrobiologia 587.1 (Aug. 2007), pp. 5-18. DOI: 10 . $1007 / \mathrm{s} 10750-007-0675-9$.

[120] Partha Sarathi et al. "Experimental Measurements of Expected Mass Fraction in a Contaminant Plume". In: Boundary-Layer Meteorology 137.2 (Nov. 2010), pp. 167-185. DOI: 10.1007 /s10546-010-9526-8.

[121] P. Sarathi et al. "A calibration scheme for quantitative concentration measurements using simultaneous PIV and PLIF". In: Experiments in Fluids 52 (Jan. 2012), pp. 247-259. DOI: 10.1007 /s $00348-011-1219-7$.

[122] Hubert H.G. Savenije. "Prediction in ungauged estuaries: An integrated theory". In: Water Resources Research (2015), pp. 2464-2476. DOI: 10 . 1002 / 2015WR016936. Received.

[123] Hubert H.G. Savenije. Salinity and tides in alluvial estuaries. Amsterdam: Elsevier, 2005, p. 194.

[124] B.L. Sawford and P.J. Sullivan. "A simple representation of a developing contaminant concentration field". In: Journal of Fluid Mechanics 289 (1995), pp. 141-157. DOI: 10.1017 /S0022112095001273.

[125] T.P. Schopflocher. The representation of the scalar concentration PDF in turbulent flows as a mixture. 1999. 
[126] T.P. Schopflocher, C.J. Smith, and P.J. Sullivan. "Scalar concentration reduction in a contaminant cloud". In: Boundary-Layer Meteorology 122.3 (2007), pp. 683-700. DOI: 10.1007 /s10546-006-9126-9.

[127] T.P. Schopflocher and P.J. Sullivan. "A mixture model for the PDF of a diffusing scalar in a turbulent flow". In: Atmospheric Environment 36.27 (Sept. 2002), pp. 4405-4417. DOI: $10.1016 /$ S1352-2310 (02) 00287-X.

[128] Ronnie L. Schwede et al. "Impact of sampling volume on the probability density function of steady state concentration". In: Water Resources Research 44.12 (2008), pp. 1-16. DOI: $10.1029 / 2007$ WR0 06668.

[129] Claude E. Shannon. "A mathematical theory of communication". In: The Bell System technical journal 27 (1948), pp. 379-423.

[130] J.H. Simpson et al. "Tidal Straining, Density Currents, and Stirring in the Control of Estuarine Stratification". In: Estuaries 13.2 (1990), p. 125. DOI: $10.2307 / 1351581$.

[131] John Skilling. “Classic maximum entropy”. In: Maximum entropy and Bayesian methods. Springer, 1989, pp. 45-52.

[132] Alex Smajgl and John Ward. "Evaluating participatory research: Framework, methods and implementation results". In: Journal of Environmental Management 157.Supplement C (2015), pp. 311-319. DOI: 10 . 1016 / j . jenvman.2015.04.014.

[133] C.J. Smith. "Scalar concentration reduction in a contaminant cloud". PhD thesis. University of Western Ontario, 2004.

[134] V.H. Smith, G.D. Tilman, and J.C. Nekola. "Eutrophication: impacts of excess nutrient inputs on freshwater, marine, and terrestrial ecosystems". In: Environmental Pollution 100.1-3 (1999), pp. 179-196. DOI: 10 . 1016 / s0269-7491 (99) 00091-3.

[135] G. Srinivasan et al. "Random walk particle tracking simulations of nonFickian transport in heterogeneous media". In: Journal of Computational Physics 229.11 (2010), pp. 4304-4314.

[136] Veljko Srzic. "Significance of transport dynamics on concentration statistics and expected mass fraction based risk assessment in the subsurface". PhD thesis. KTH Royal Institute of Technology, 2013.

[137] Veljko Srzic et al. "Impact of aquifer heterogeneity structure and localscale dispersion on solute concentration uncertainty". In: Water Resources Research 49.6 (2013), pp. 3712-3728. DOI: 10.1002 /wrcr. 20314. 
[138] P.J. Sullivan. "Instantaneous velocity and length scales in a turbulent shear flow". In: Advances in Geophysics 18 (1975), pp. 213-223.

[139] P.J. Sullivan. "The dilution of a contaminant substance released in an environmental flow". In: The Indian Journal of Statistics 57 (1995), pp. 277-284.

[140] P.J. Sullivan. "The influence of molecular diffusion on the distributed moments of a scalar PDF". In: Environmetrics 15.2 (2004), pp. 173-191. DOI: $10.1002 /$ env. 633.

[141] P.J. Sullivan and H. Ye. "Further comments on "cloud-averaged" concentration statistics". In: Mathematics and Computers in Simulation 35.3 (Sept. 1993), pp. 263-269. DOI: 10 .1016/0378-4754(93) 90004-E.

[142] P.J. Sullivan and H. Ye. "Moment inversion for contaminant concentration in turbulent flows". In: Canadian Applied Mathematics Quarterly 4.3 (1996), pp. 301-310.

[143] P.J. Sullivan and H. Ye. "The need for a new measure of contaminant cloud concentration reduction". In: Nuovo cimento della Società italiana di fisica. C 20.3 (1997), pp. 413-423.

[144] Marina Tavra, Niksa Jajac, and Vlado Cetl. "Marine Spatial Data Infrastructure Development Framework: Croatia Case Study". In: ISPRS International Journal of Geo-Information 6.4 (2017), p. 117. DOI: 10 . 3390 / i jg i 6040117.

[145] Keith R. Thompson et al. "Probabilistic characterization of tidal mixing in a coastal embayment: a Markov Chain approach". In: Continental Shelf Research 22.11 (2002), pp. 1603-1614. DOI: 10.1016 / S0278-4343 (02) $00024-9$.

[146] Josko Troselj et al. "Modeling of extreme freshwater outflow from the north-eastern Japanese river basins to western Pacific Ocean". In: Journal of Hydrology 555.Supplement C (2017), pp. 956-970. DOI: 10 . 1016 / j . jhydrol.2017.10.042.

[147] Yeou-Koung Tung, Ben Chie Yen, Charles S Melching, et al. Hydrosystems engineering reliability assessment and risk analysis. McGraw-Hill New York, 2006.

[148] J.J. Vallino and Jr. C.S. Hopkinson. "Estimation of Dispersion and Characteristic Mixing Times in Plum Island Sound Estuary". In: Estuarine, Coastal and Shelf Science 46.3 (1998), pp. 333-350. DOI: 10 . 1006 / ecss . 1997. 0281. 
[149] Nuno Vaz, João Miguel Dias, and Paulo Chambel Leitão. “Three-dimensional modelling of a tidal channel: the Espinheiro Channel (Portugal)". In: Continental Shelf Research 29.1 (2009), pp. 29-41. DOI: 10 . $1016 /$ j . csr. 2007.12 .005$.

[150] Peter M. Vitousek et al. "Human Alteration of the Global Nitrogen Cycle: Sources and Consequences". In: Ecological Applications 7.3 (1997), pp. 737750. DOI: 10 . 1890/1051-0761 (1997) 007 [0737: HAOTGN] 2 . 0 . CO ; 2.

[151] Jianfeng Wang et al. "The Effect of Discharge, Tides, and Wind on Lift-Off Turbulence". In: Estuaries and Coasts 38.6 (2015), pp. 1-15. DOI: 10.1007 / s12237-015-9958-y.

[152] Jiangtao Xu et al. "Climate Forcing and Salinity Variability in Chesapeake Bay, USA". In: Estuaries and Coasts 35.1 (2012), pp. 237-261. DOI: 10 . 1007 / s12237-011-9423-5.

[153] Ping Yang et al. "Dynamics of dissolved nutrients in the aquaculture shrimp ponds of the Min River estuary, China: Concentrations, fluxes and environmental loads". In: Science of the Total Environment 603 (2017), pp. 256267.

[154] Honglin Ye. "A new statistic for the contaminant dilution process in turbulent flows". PhD thesis. University of Westerm Ontario, 1995. 


\section{Personal information:}

- Morena Galešić

- Tršćanska 54, 21000 Split (Hrvatska)

- morena.galesic@gradst.hr

- Date of birth: 30 th December 1987.

\section{Education:}

- 2002.-2006. General - education high school, Biograd n/m

- 2006.-2009. Undergraduate study of civil engineering at Faculty of Civil Engineering, Architecture and Geodesy Split, University of Split

- 2009.-2011. Graduate study of civil engineering at Faculty of Civil Engineering, Architecture and Geodesy Split, University of Split

Work experience:

- 2011.-2012. Project Manager Assistant for „Optimization of Utilization of Drinking Water Distribution Network - VIK Split “; SAFEGE Croatia d.o.o., Zagreb

- 2015.-2016. Project Manager for "Risk Assessment of the pollution caused by rivers and discharges in coastal area" Coastal Pollution Risk Tool (CPoRT)

- 2012. - 2018. Research and Teaching Assistant at Faculty of Civil Engineering, Architecture and Geodesy Split, University of Split List of publications:

\section{Journal Article (Web of Science)}

1. Andričević, R., Galešić, M.: "Contaminant dilution measure for the solute transport in an estuary", Advances in Water Resources (2018), under review. 
2. Galešić, M., Andričević, R., Gotovac, H., Srzić, V.: "Concentration statistics of solute transport for the near field zone of an estuary", Advances in Water Resources. 94 (2016); 424-440.

3. Galešić, M., Gotovac, H.: "Potential impact of heterogeneity on groundwater age", Water Science \& Technology: Water Supply. 14 (2014), 3; 398-404.

\section{Conference proceeding articles}

1. Galešić, M., Andričević, R., Divić, V.: Integrated concentration statistics from river generated pollution in coastal zones, $4^{\text {th }}$ Coastal \& Maritime Mediterranean Conference $C M^{2}$, Split, Croatia, 29. November-02. December, 2017.

2. Galešić, M., Andričević, R., Divić, V.: Estimation of statistical parameters of nutrients concentration generated in estuaries, Common foundation 2017 (fifth conference of young scientists in Croatia and region in field of civil engineering and related fields), Zagreb, Croatia, 18.-19. September 2017. / Zagreb: Faculty of Civil Engineering, University of Zagreb, 2017. 111-117.

3. Galešić M, Andričević R, Divić V, Šakić Trogrlić R: "New screening tool for obtaining concentration statistics of pollution generated by rivers in estuaries", 15th International Conference on Environmental Science and Technology, Rhodes, Greece, 31. August-02. September 2017.

4. Galešić, M., Andričević, R., Divić, V., Mateus, M., Pinto, L.: "Potential data used for validation of concentration statistics obtained using analytical model for conservative transport in an estuary", European Geosciences Union Conference, Vienna, Austria, 18.-22. April 2016.

5. Galešić, M., Gotovac, H.: "Potential impact of heterogeneity on groundwater age", IWA $5^{\text {th }}$ Eastern European Young and Senior Water Professionals Conference, Kiev, Ukraine, 26.-28. June 2013. 


\section{Other publications}

1. Galešić, M.: Pollution transport modelling in an estuary, Common foundation 2014 (second conference of young scientists in Croatia and region in field of civil engineering and related fields), Rijeka, Croatia, 24.-26. September 2014. / Marović, I.; Sušanj, I. Rijeka: Faculty of Civil Engineering, University of Rijeka, 2014. 28-29.

2. Crupi, V., Dima, S. M., Galešić, M., Mihalca, I. A., Palenčíková, Z., Yadate, D. M.: "DeWOS (Danube Wellbeing Operation System) The Development of a Decision Support System for Wellbeing in the Danube River Basin", DIAnet International School Proceedings 2013. Interdisciplinary Methods for the Sustainable Development of the Danube Region. Gorizia, 13rd - 22nd April 2013 / Brumat, S.; Frausin, D. Trst, Italija: EUT Edizioni Università di Trieste, 2013. 116-129.

3. Galešić, M., Gotovac, H.: "Heterogeneity influence on groundwater age", International Journal for Engineering Modelling (2012), Vol.25, No. 1-4 (preliminary report).

\section{Memberships}

Morena Coaching International (MCI)

Water Youth Network (WYN)

Coastal and Estuarine Research Federation (CERF) 